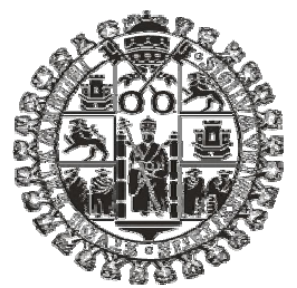

Department of Microbiology and Genetics Institute of Biochemical Microbiology

\title{
Transcriptional analysis of the histone acetylation and deacetylation in Candida albicans
}

\author{
Ph.D Thesis \\ Ahmad Rashki
}

\author{
Ph.D Thesis Adviser \\ Prof. Dr. Angel Dominguez Olavarri \\ Department of Microbiology and Genetics, University of Salamanca
}


Prof. Dr. Angel Dominguez Olavarri Professor and Chair of Microbiology at the Department of Microbiology and Genetics, University of Salamanca

\section{CERTIFY:}

That the present dissertation entitled "Transcriptional analysis of the histone acetylation and deacetylation in Candida albicans", submitted to apply for Ph.D degree, has been carried out by Ahmad Rashki under my direction in the Department of Microbiology and Genetics, University of Salamanca.

Prof. Dr. Angel Dominguez Olavarri Professor and Chair 
Prof. Dr. Angel Dominguez Olavarri, Head of the Department of Microbiology and Genetics of the University of Salamanca

\section{CERTIFY:}

That the present dissertation entitled "Transcriptional analysis of the histone acetylation and deacetylation in Candida albicans", submitted to apply for Ph.D degree, has been carried out by Ahmad Rashki under direction Prof. Dr. Angel Dominguez Olavarri in the Department of Microbiology and Genetics, University of Salamanca.

Prof. Dr. Angel Dominguez Olavarri Head of the Department of Microbiology and Genetics 
The project work has been supported in part by grants:

DGICYT "Identificación de factores de virulencia y nuevas dianas terapéuticas en Candida albicans mediante análisis funcional del genoma y del proteoma" (Proyecto BIO2002-02124)

Unión Europea. "Interaction of fungal pathogens with host cells: a post genomic approach" dentro del Progama Marie Curie Research Training Network (MRTN-CT2003-504148)

DGICYT "Analisis de las interacciones de Candida albicans con el hospedador: modelos "ex vivo" e "in vivo".

(Proyecto SAF2005-07131)

FIS "Analisis de los mecanismos moleculares de infecciones fungicas mediante aproximaciones genomicas y proteomicas. Establecimiento de modelos animales." (Proyecto PI 050849).

"Glycoshield: surface modulation of the fungal and host response using a genomic approach". Proyectos de investigación en el marco de las redes del Espacio Europeo de Investigación (esquema ERA-NET). GEN2006-27775-C2-E/PAT

Junta de Castilla y León "Histona acetyltransferasas en Candida albicans efecto sobre dimorfismo y virulencia. Una aproximación Post-genomica" (SA 141A08) 
Read: In the name of thy Lord who createth, Createth man from a clot. Read: and thy Lord is the most bounteous, Who teacheth by the pen, Teacheth man that which he knew not. (Holy Qur'an)

Dedicated to my parents, to whom I owe the gift of life. 


\section{INDEX}

Acknowledgements

List of tables

List of figures

Introduction

General biology of Candida albicans ........................................................

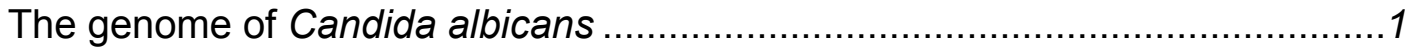

Candida albicans as a pathogenic agent ..................................................

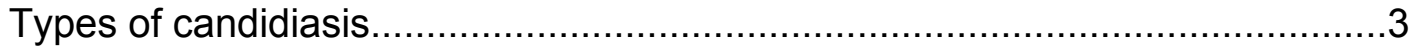

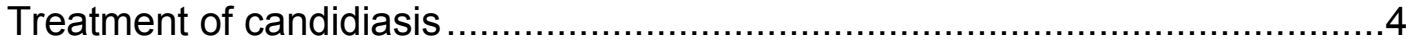

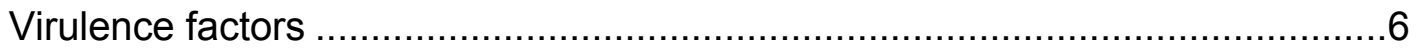

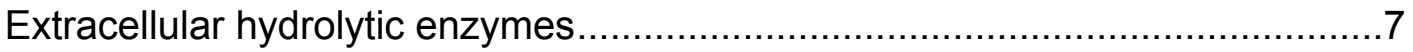

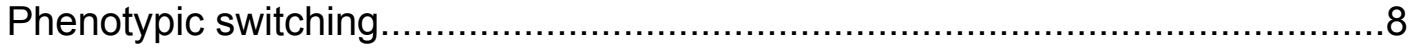

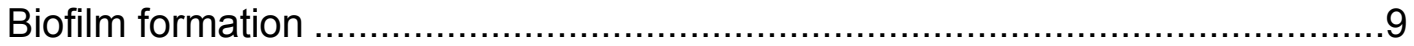

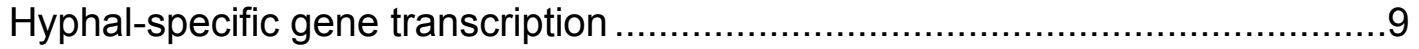

Regulation of central metabolic pathways in a fungal pathogen.......................10

Virulence phenotypes and the choice of animal model ..................................11

The actin cytoskeleton is required for the dimorphic switch in

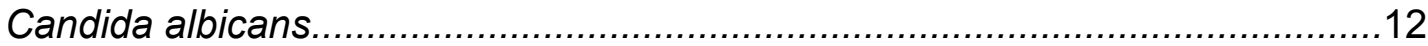

Transcriptional control and chromatin remodelling proteins ...........................12

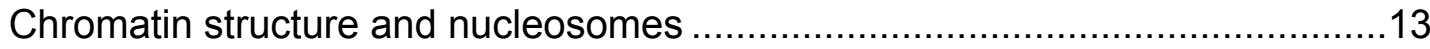

Histone acetylation and transcriptional control ...........................................15

HATs serve a multiplicity of functions ........................................................16

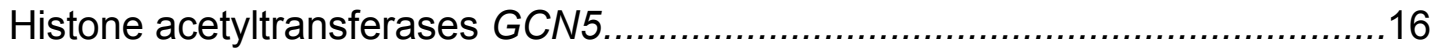

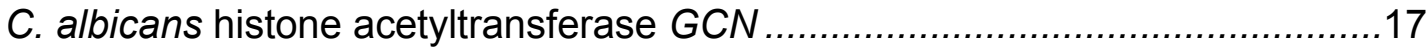

HAT's and their biological functions ......................................................... 19

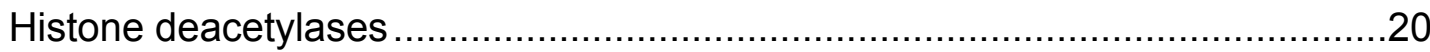

Crosstalk between histone acetylation/deacetylation and chromatin

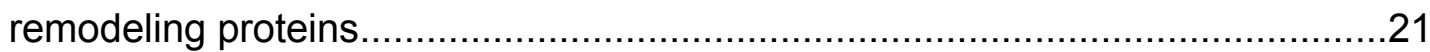

cDNA microarray analysis of differential gene expression in C. albicans..........22

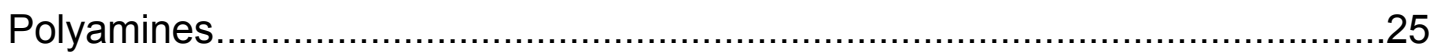

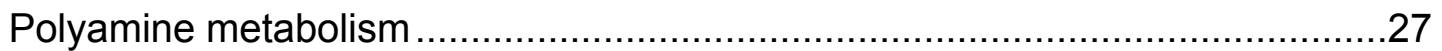

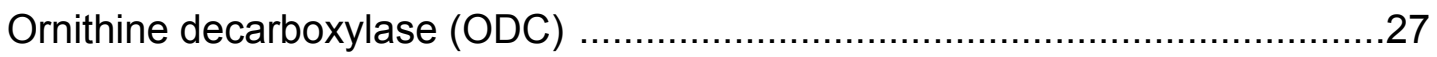


Function of polyamines in eukaryotic cells.

Polyamine auxotrophs of Candida albicans.

\section{Chapter II}

Transcriptional response of Candida albicans to temperature

\section{Chapter III}

Phenotypic, virulence and transcriptome analysis of the Candida albicans gcn5, hda1 double mutant: Comparison with the gcn5 and hda1 simple mutants

\section{Chapter IV}

Global transcriptome of Candida albicans odc null mutant during the yeast mode of growth and during the yeast-hypha transition

\section{Chapter V}

Analysis of Candida albicans mutant lacking the histone acetyltransferases, GCN5 and HAT1

\section{ChapterVI}

Spanish summary

Conclusiones 169

Reference list .172 


\section{Acknowledgements}

I would like to express my sincerest appreciation to my advisor, Prof. Dr. Angel Dominguez Olavarri, who believed in me and gave me the chance to start my project in his lab. He trained me to think deeply and thoroughly about research work. He always encouraged me to aim high and work hard to explore the scientific fields. I couldn't have completed this work without his support, advice, guidance, understanding and encouragement. His dedication to science is a great motivation for me to pursue a scientific career.

I appreciate Dr. Maria Carmen Lopez for her helpful advice on molecular biology techniques.

I give special thanks to Dr. Nuria Martin Laso who worked hard to help with my microarray experiments. She was always very patient in helping me, solve problems and giving me suggestions of a beginner.

I am thankful to my colleagues and lab members: Laura Mojardin, Esperanza, Rosa, Ivan, Pilar, Rita, Carlos, Laura, Azul, Ana, Adam and Juan.

I would like to express my deepest gratitude to Professor Carlos Rodriguez Vazquez De Aldana for his friendly assistance in every difficult moment of my work.

I am very grateful to Dr. Alberto Gonzalez-Novo and Dr. Fransisco Conde Perez, for effective and intelligent suggestions.

Many thanks also to Dr. Manuel Sanchez Fernandez for DNA sequencing.

Finally, I thank to my doughters, Mahsa and Rozhan, and my wife. They give me tremendous support and encouragement throughout my graduate study. 


\section{List of tables}

Table1.1 Some of example about cDNA microarray analysis of differential gene expression in C. albicans

Table 2.1 Genes whose transcription is induced more than 1.5 fold at $37^{\circ} \mathrm{C}$ at the three times assayed, 15, 60 and $180 \mathrm{~min}$ 38

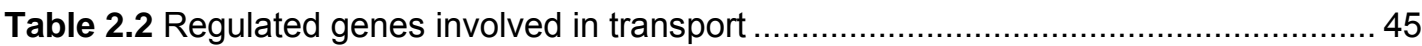

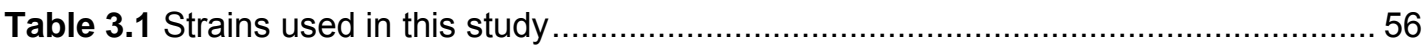

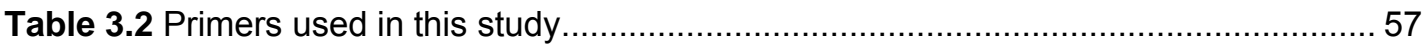

Table 3.3 Differentially expressed genes in the gcn5, hda1 mutant during the yeast mode of growth

Table 3.4 Differentially expressed genes during the three times of incubation in the double mutant strain 71

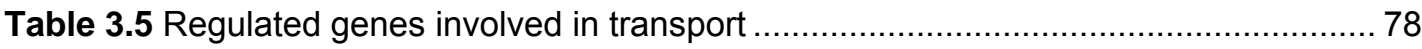

Table 4.1 Distribution of differentially expressed genes during the yeast mode growth into functional categories genes

Table 4.2 Distribution of differentially expressed genes during the three times $(15,60$ and $180 \mathrm{~min})$ of yeast-hypha transition form into functional categories 94

Table 5.1 strains used in this study 114

Table 5.2 Primes used in this study 115

Table 5.3 Functional classifications of differentially expressed genes during the yeast mode of growth in the gcn5, hat 1 double mutant..... 123 


\section{List of figures}

Figure 1.1 Growth of C. albicans yeast (A), hypha (B), pseudohypha(C) and chlamydospore

Figure 1.2 Schematic diagrams illustrating the contribution of the virulence attributes to $C$. albicans pathogenicity

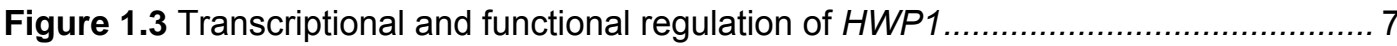

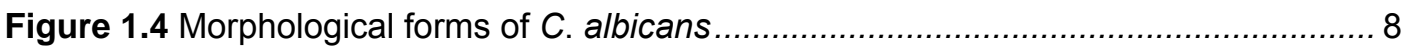

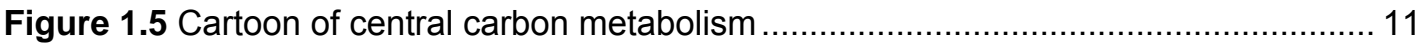

Figure 1.6 (A) Nucleosome structure. (B) The crystal structure ...................................... 13

Figure 1.7 Diagram representing the Hat and Bromodomain of C. albicans GCN5 ........... 17

Figure 1.8 Dendograms representing the phylogenetic relationships of $C$. albicans GCN5 18

Figure 1.9 Pathway of polyamine synthesis in eukaryotic organisms ................................ 26

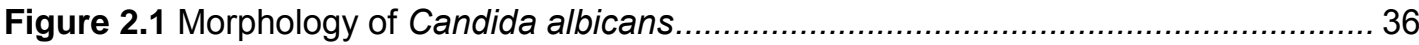

Figure 2.2 Total number of genes regulated by temperature at the $\geq 1.5$-fold level ............ 36

Figure 2.3 Genes regulated by temperature. (A) Venn diagram of up-regulated and down-regulated genes, including those common to all three times studied. ....37

Figure 2.4 Transcriptional regulation by temperature. Cell wall, $(A)$ and 42

Figure 2.5 Transcriptional regulations by temperature of genes involved in glycolysis and gluconeogenesis (A) and TCA (B) and glyoxylate (C)cycles

Figure 2.6 Transcriptional regulation by temperature of genes involved in transporters, (A) drug resistance, 46

Figure 3.1 Mapping of GCN5 transcription initiation sites by RLM-RACE ........................6 60

Figure 3.2 Southern blot analysis of the SGM4 double mutant .....................................6 60

Figure 3.3 Growth curves of CAI4, NDH4 (hda1), RDG4 (gcn5) and SGM4 ...................... 61

Figure 3.4 (A) Colony growth and (B) morphological characteristics of C. albicans............ 61

Figure 3.5 Phenotypes of the CAl4, NDH4 (hda1), RDG4 (gcn5) and ............................... 62

Figure 3.6 Cellular morphology of CAI4 and SGM4 (gcn5, hda1) strains .........................63

Figure 3.7 Chlamydospore formation in C. albicans CAl4, NDH4 (hda1)

RDG4 (gcn5) and SGM4 (gcn5, hda1) mutant.

Figure 3.8 Sensitivity assays of CAI4, NDH4 (hda1), RDG4 (gcn5) and SGM4 ................. 64

Figure 3.9 Resistance to a cell wall-degrading enzymatic complex...................................... 64

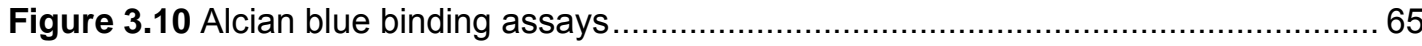


Figure 3.11 Virulence assays

Figure 3.12 Transcript profiles of the C. albicans gcn5 and hda 1 single and double mutants during the yeast growth form (A). Venn diagrams of genes regulated by Gcn5p and Hda1p in the single and double mutant strains

Figure 3.13 Transcript profiles of $C$. albicans double mutant during the yeast-hypha transition (15, 60 and $180 \mathrm{~min})(\mathbf{A})$. Venn diagrams 70

Figure 3.14 Transcriptional regulation of genes involved in the cell wall in 74

Figure 3.15 Transcriptional regulations of genes coding for ribosomal proteins 76

Figure 3.16Transcriptional regulation of genes involved in the glycolytic pathway 77

Figure 3.17 Differential expressions of the genes involved in sulphur amino acid 79

Figure 3.18 Sphingolipid biosynthesis pathway in the yeast. 80

Figure 4.1 A) Transcriptional Genes Tree and statistical comparisons of polyamines-responsive genes in a $C$. albicans odc/odc mutant strain 89

Figure 4.2 Transcriptional regulations of individual genes involved in carbohydrate metabolism

Figure 4.3 Transcriptional regulations of individual genes involved in respiration 99

Figure 4.4 Transcriptional regulations of genes encoding for transcription factors during the three times of incubation by $C$. albicans odc/odc mutant 101

Figure 4.5 Transcriptional regulation of genes involved in DNA-directed RNA polymerase

(A), nucleolar proteins 102

Figure 4.6 Transcriptional regulations of genes involved in secretory pathway and 104

Figure 5.1 Deletion of CaHAT1 alleles (A) Structure of different alleles..... 116

Figure 5.2Cellular morphology of CAI4, RDG4, RGL4 and AHM4 strain 116

Figure 5.3 Growth curves of the mutant strains 117

Figure 5.4 Phenotypes of the CAI4, RDG4, RGL4 and AHM4 grown on solid 117

Figure 5.5 Morphology of CAI4, RDG4, RGL4 and AHM4 strains 118

Figure 5.6 A chlamydospore formation of $C$. albicans is defective in gcn5, hat1 119

Figure 5.7 Virulence assays 119

Figure 5.8 Resistance to a cell wall-degrading enzymatic complex of C. albicans...... 120

Figure 5.9 Sensibility assays of CAI4, RDG4 (gcn5), RGL4 (hat1) and 121

Figure 5.10 Cell surface charge in AHM4 (gcn5, hat1) strain. 121 


\section{List of abbreviations}

\begin{tabular}{|c|c|}
\hline bp & base pair \\
\hline CFW & Calcofluor white \\
\hline $\mathrm{CaCl}_{2}$ & Calcium Chloride \\
\hline cDNA & Complementary Deoxyribonucleic acid \\
\hline CIP & Calf Intestine Alkaline Phosphatase \\
\hline Су3 & Cyanine Dye 3 \\
\hline Cy5 & Cyanine Dye 5 \\
\hline dCTP & Deoxycytidinetriphosphate \\
\hline DNA & Deoxyribonucleic Acid \\
\hline dNTP & Deoxynucleosidetriphosphate \\
\hline E. coli & Escherichia coli \\
\hline f.o.i & Frequency of incorporation \\
\hline GICNAC & $\mathrm{N}$-acetylglucosamine \\
\hline $\mathbf{h}$ & Hour \\
\hline HAT & Histone Acetyltransferase \\
\hline HDAC & Histone Deacetylase \\
\hline $\mathbf{K b}$ & Kilobase \\
\hline LB & Lauria Bertani \\
\hline M & Molar \\
\hline $\min$ & Minute \\
\hline ml & Millilitre \\
\hline $\mathrm{mM}$ & Millimolar \\
\hline mRNA & Messenger ribonucleic Acid \\
\hline $\mathrm{NaCl}$ & Sodium Chloride \\
\hline ng & nanogram \\
\hline $\mathrm{nm}$ & nanometer \\
\hline OD & Optical Density at specific wavelength \\
\hline ORF & Open Reading Frame \\
\hline PCR & Polymerase Chain Reaction \\
\hline Pmol & Picomol \\
\hline RNA & Ribonucleic Acid \\
\hline RNAse & Ribonuclease \\
\hline rpm & revolution per minute \\
\hline rRNA & Ribosomal Ribonucleic Acid \\
\hline RT & Room temperature \\
\hline SDS & Sodium dodecyl sulphate \\
\hline SSC & Sodium Chloride-Sodium Citrate \\
\hline TE & Tris, EDTA \\
\hline TRIS & Tris-(hydroxymethyl)-aminomethane \\
\hline Ufc & Unit forming colony \\
\hline UV & Ultra Violet \\
\hline$\mu \mathbf{g}$ & Microgram \\
\hline$\mu \mathrm{l}$ & Microlitre \\
\hline$\mu \mathrm{m}$ & Micromolar \\
\hline${ }^{\circ} \mathrm{C}$ & Degree Centigrade \\
\hline
\end{tabular}




\section{General biology of Candida albicans}

Candida albicans is an important human pathogen that displays a remarkable ability to detect changes in the environment and to respond appropriately by changing its cell morphology and physiology [40]. C. albicans is a pleomorphism diploid fungus which presents the ability to grow under a variety of morphological forms that include unicellular budding yeast, also known as blastospore, true hypha and pseudohyphal forms. C. albicans also can form chlamydospores that is to say; asexual spores which develop over pseudohyphal support cells and appear under unfavourable environmental conditions (Figure 1.1) [254,451].

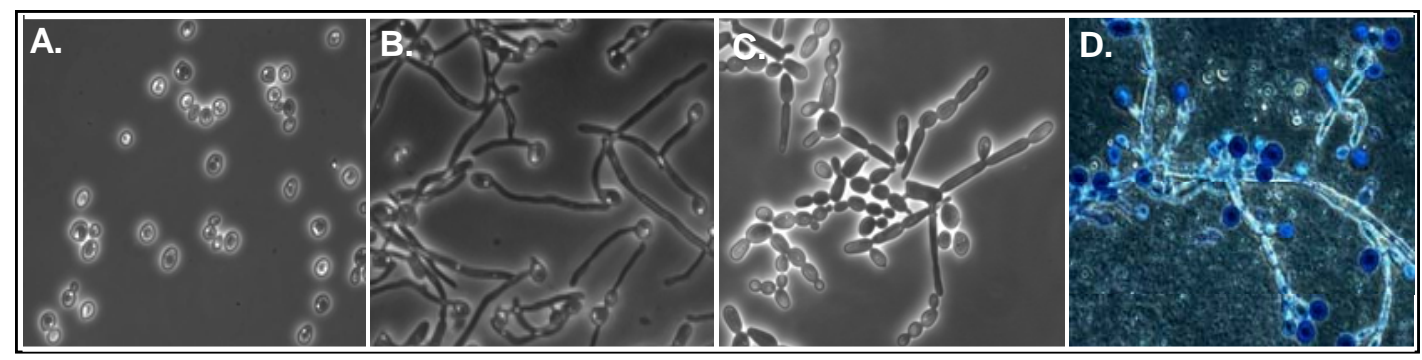

Figure 1.1 Growth of C. albicans yeast (A), hypha (B), pseudohypha (C) and chlamydospore (D)

Fungi are classified on the basis of their ability to reproduce sexually, asexually or by a combination of both. Thus, $C$. albicans has traditionally been classified in the Deuteromycota (fungi imperfecti) since the sexual phase of this fungus is unknown, although recent reports described the presence of mating-type-like orthologs (MTL) in C. albicans of both of the Saccharomyces cerevisiae mating-type genes (MAT), a and $\alpha[213,296]$. Therefore, C. albicans has been now classified as follows:

Phylum Ascomycota

Subphylum Ascomycotina

Class. Ascomycetes

Order Saccharomycetales

Family. Saccharomycetaceae

Genus Candida

\section{The genome of Candida albicans}

The hemiascomycete $C$. albicans is an opportunistic pathogen causing human fungal infections, mainly in immunocompromised hosts. The complete sequence of 
the C. albicans genome has been determined (http://wwwsequence.stanford.edu/group/candida) based on a shotgun strategy carried out at the Stanford Genome Technology Center. The total assembly of the haploid genome comprises $\sim 16 \mathrm{Mb}$ (approximately 33\% larger than that of $\mathrm{S}$. cerevisiae) corresponding to approximately 6244 annotated entries corresponding to 130 tRNA genes and 5917 protein-coding genes that annotated by the European Consortium Galar Fungail (http://genolist.pasteur.fr/CandidaDB) $[75,96]$. This nuclear genome distributed in eight pairs of homologous chromosomes which are numbered from 1 (largest) to 7 (smallest), with the one carrying the ribosomal DNA called R [502]. CandidaDB release 2.0 contains information pertaining to Assembly 19 of the genome of C. albicans strain SC5314 [96].

Assembly 19, is distributed over 412 supercontigs, of which 266 constitute a reference haploid genome of $14855 \mathrm{~kb}$ and 146 constitute allelic counterparts of supercontigs included in the reference haploid genome [224]. The reference haploid genome contains 7677 ORFs of 100 codons or longer, and a reduced set of 6419 ORFs has been derived by eliminating the smaller of a pair of ORFs that overlap by more than 50\% [224]. Assembly 21 reveals an ancient chromosome fusion, a number of small internal duplications followed by inversions, and a subtelomeric arrangement, including a new gene family, the TLO genes. Correlations of position with relatedness of gene families imply a novel method of dispersion. The sequence of the individual chromosomes of $C$. albicans raises interesting biological questions about gene family creation and dispersion, subtelomere organization and chromosome evolution [482].

\section{Candida albicans as a pathogenic agent}

Candida spp. are the fourth leading cause of nosocomial bloodstream infections in the United States, with treatment costs estimated to be more than $\$ 2-\$ 4$ billion annually [506] and with attributable mortality rates estimated to be between $38 \%$ to $49 \%$ [168]. C. albicans can grow as budding yeast, in a pseudohyphal form or as true hypha depending upon the environmental conditions [451]. Growth is polarized in $C$. albicans hypha, with continuous apical growth throughout the cell cycle and parallel cell walls at the septal junctions. In contrast, the growth of pseudohypha and blastophores is only limited to the apical tip during the initial part of the cell cycle [76]. C. albicans pseudohypha has a constriction at the septal junction 
between mother and daughter cells. Pseudohypha can be distinguished from true hypha on the basis of their morphological index which quantifies the dimensions of cell compartments. Alternatively they can be distinguished on the basis of the positions of their septal junctions. These lie at the bud neck for pseudohypha, and within the germ tube for emerging hypha [451]. The yeast-hyphal transition is regulated by a complex network of signalling pathways that include evolutionarily conserved MAP kinase and Ras-cAMP modules [122,157,451,501]. The yeast-like form is thought to promote dissemination within the blood stream whereas hyphal development appears to promote invasion $[157,451,456]$. The link between specific morphotypes and virulence is not firmly established [157]. However, hypha is important for the formation of biofilm, which can provide reservoirs of infection in the host, and morphogenetic variation is likely to be important in responding rapidly to environmental change and to host defence mechanisms [358]. Generally, growth of $C$. albicans in the yeast form is favoured at high cell densities, temperatures below $30^{\circ} \mathrm{C}$ and ambient $\mathrm{pH}$ below 4 . In contrast, the hyphal form is favoured at temperatures above $37^{\circ} \mathrm{C}$, at ambient $\mathrm{pH}$ close to 7 , at lower cell densities, at high $\mathrm{CO}_{2}$ concentrations, under some limiting nutrient conditions such as amino acid starvation or hypoxia, or in the presence of serum [120,239,358,472]. The effect of serum is complex but it is proposed to act, in part, by conferring amino acid starvation [472] although other factors are implicated [212,299].

\section{Types of candidiasis}

Candidiasis can range from superficial disorders to invasive infections, in general in immunocompromised hosts, and usually are classified into two big groups as follow superficial candidiasis, which may involve the epidermal and mucosal surfaces, including those of the oral cavity, pharynx, oesophagus, intestines, urinary bladder, and vagina. Symptomatic mucosal and superficial candidiasis arises in subjects colonized with Candida who are predisposed by illness, debility, or a local reduction in host resistance to an overgrowth of their own indigenous flora [358,485], and the different forms of superficial candidiasis are often associated with biofilm formation on surfaces of host tissues or medical devices [415]. In patients with probable immunodeficiency or visible candidiasis of the skin, oropharynx, or esophagus, a Candida infection should be considered in case of a gastrostomy site infection [511]. 
Systemic candidiasis, considerable changes in the relative frequency of systemic infections due to various Candida species as well as their in vitro susceptibility patterns have been noted in several parts of the world [401]. It is widely known that systemic candidiasis caused by Candida albicans strains endangers the lives of hospitalised patients since these pathogens are extremely difficult to defeat by commonly used antifungal agents [417].

Candida albicans may affect the kidneys, liver, spleen, brain, eyes, heart and other tissues the principal risk factors predisposing to deeply invasive candidiasis (Figure 1.2) are protracted courses of broad spectrum antibiotics, cytotoxic chemotherapy, corticosteroids, HIV and vascular catheters [230].

1. Colonization

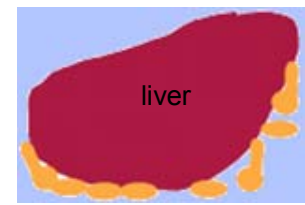

3. Profound infection

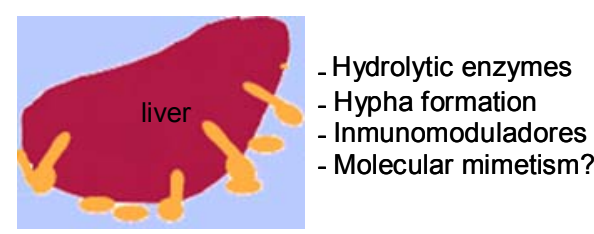

2. Superficial infection

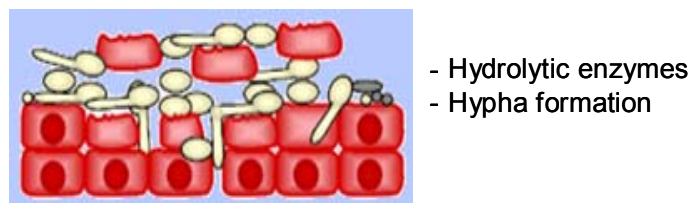

4. Disseminated infection

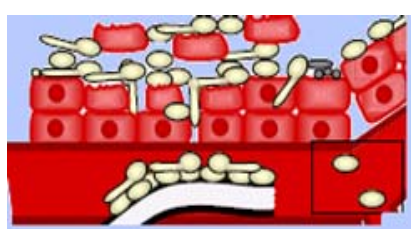

- Adhesion

- Hydrolytic enzymes

- Dimorfismo

- Switching phenotypic

- Antioxidantes?

- Inmunomoduladores

Figure 1.2 Schematic diagrams illustrating the contribution of the virulence attributes to $C$. albicans pathogenicity. C. albicans commonly colonizes the epithelial surface (stage 1) and causes superficial infections (stage 2 ), but under conditions when the host is compromised, the fungus establishes deep-seated infections (stage 3) by penetrating further into the epithelial tissue. Occasionally, C. albicans causes disseminated infections (stage 4), which allow the fungus to colonize and infect other host tissues and can be fatal.

\section{Treatment of candidiasis}

Invasive fungal infections are an important cause of morbidity and mortality, particularly in patients with underlying risk factors e.g. neutropenia, cancer chemotherapy, transplantation and AIDS [74]. C. albicans is a eukaryotic organism and therefore share many of its biological processes with human cells; most antifungal drugs cause deleterious side effects and, at the doses used, are fungistatic rather than fungicidal. For that reason, an important goal in antifungal drug discovery is the identification of new suitable fungal targets with no mammalian homologies. Some of the antifungal agents currently available are classified, according with their specific mode of action as follows:

- Amphotericin B. Although our antifungal armamentarium has been enlarged 
recently with new azoles (voriconazole and posaconazole) and echinocandins (caspofungin, micafungin, anidulafungin), the polyenes still have an important role in antifungal strategies because of their extended antifungal spectrum and rarity of mycological resistance [264]. This antifungal agent acts by binding to ergosterol, the principal sterol in fungal membranes, thereby perturbing membrane function to the point of causing leakage of cellular contents. Despite of the conformational difference between cholesterol and ergosterol, and the major binding affinity of Amphotericin $\mathrm{B}$ for this last one, this antifungal agent has potential toxicity for mammalian cells, so a variety of reformulated versions of the agent have been introduced in the last years, to overcome Amphotericin B nephrotoxicity by slowing the rate at which the compound is delivered to the kidneys [116].

- Azoles. The azole antifungal drugs are used to treat infections caused by Candida albicans and other fungi. These drugs interfere with the biosynthesis of ergosterol, the major sterol in fungal cells, by inhibiting an ergosterol biosynthetic enzyme, lanosterol 14 alpha demethylase, encoded by the ERG11 gene [359]. Fluconazole is effective for the prevention and treatment of candidiasis but its inactivity against fungi and increasing resistance are limiting factors. Newer azoles, particularly voriconazole and posaconazole, have an enhanced spectrum of activity that includes Candida species, Aspergillus species, Cryptococcus species, dimorphic fungi, Fusarium species, and for posaconazole, Zygomycetes. Recent data suggest that these agents are highly effective in a variety of clinical settings [74].

- Other sterol synthesis inhibitors. The increased incidence of invasive mycoses and the emerging problem of antifungal drug resistance have encouraged the search for new antifungal agents or effective combinations of existing drugs. Infections due to Candida albicans are usually treated with azole antifungals such as Fluconazole, Ketoconazole or Itraconazole. Whilst azoles may have little or no toxicity, they generally offer rather poor fungicidal activity. Even in the absence of resistance, treatment failures or recurrent infections are not uncommon, especially in immunocompromised individuals. Non-classical antifolate pyrimethamine shows synergy with azole antifungal compounds and interferes with the ergosterol biosynthesis pathway in $C$. albicans. By disturbing folate metabolism in this fungus, pyrimethamine can inhibit ergosterol production [344]. There are two other classes of antifungal agents that acts at this same pathway, allylamines, which act by 
inhibition of squalene epoxidase, with fungicidal consequences in many filamentous fungi but not so much effective in pathogenic yeasts, and morpholines, affecting two different enzymes in the ergosterol pathway, Erg24p and Erg2p.

- Equinocandins. Echinocandins have good activity against Candida species and Aspergillus species but their spectrum generally does not include Fusarium, Cryptococcus, Trichosporon, Zygomycetes and Dematiaceous fungi. Their target is the complex of proteins responsible for synthesis of cell wall $\beta-1,3$ glucan polysaccharides, and the component to which equinocandins bind is Fsk1p, although the complete mechanism of the action still remains unknown $[481,513]$.

\section{Virulence factors}

The virulence of Candida species depends on many environmental conditions including extracellular $\mathrm{pH}$ concentration of alkali metal cations [246], expression of host recognition molecules or adhesins [138,422,540], cell wall organization and growth rate [534] and expression of tissue invasion-facilitating enzymes, such as phospholipases and secreted aspartyl proteinases [127,147,242,337,406].

\section{Adhesins}

The fungal cell surface contributes to pathogenesis by mediating interactions with host cells and eliciting host immune responses [343]. The virulence factors expressed or required by Candida species, and in particular by $C$. albicans, vary depending on the type of the infection (e.g. mucosal or systemic), the site and stage of infection, and the nature of the host response [123]. One of the important virulence attributes is adhesion. Most studies focus on the role of two wellcharacterized adhesins (ALS family and Hwp1p) in morphogenesis, pathogenicity, and phenotype switching of C. albicans [454].

ALS family. The ALS gene family of $C$. albicans encodes large cell surface glycoproteins with a three domain structure that are implicated in the process of adhesin to host surfaces [200].

Hwp1p. This protein encodes an outer mannoprotein, with a cell surface-exposed $\mathrm{NH}_{2}$-terminal domain and $\mathrm{COOH}$-terminal features conferring covalent integration into cell wall $\beta$-glucan. It is conditionally required for hyphal formation: the ability to form hypha on solid media is severely reduced in an HWP1 heterozygous mutant 
and eliminated in the null mutant. In the presence of serum, colonies of the null mutant produce peripheral hypha but at reduced levels compared to the wild type. This protein encodes an outer surface mannoprotein that is believed to be oriented with its amino-terminal domain surface-exposed and the carboxyl terminus, most probably covalently integrated with cell wall $\beta$-glucan. The amino-terminal sequence of Hwp1p was found to resemble mammalian transglutaminase substrates suggesting that Hwp1p is involved in the formation of stable complex between germ tubes (Figure 1.3) (initial projections observed when Candida switches from yeast form to hyphal growth) [443].

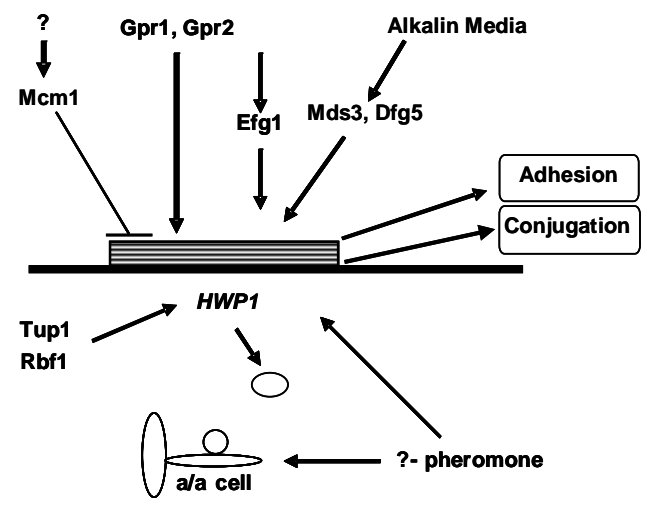

Figure 1.3 Transcriptional and functional regulation of HWP1 [34]

HWP1 is expressed during hyphal development [235,444]. Production of Hwp1p has been confirmed in the mouse model of systemic infection by immunohistochemistry [444]. More recently HWP1 expression has been observed in samples from human oral and vaginal infections by antibody and RT-PCR-based approaches $[92,338]$. These authors suggest that Hwp1p is important both for benign and invasive interactions of $C$. albicans with humans, and is also required for biofilm formation [355].

\section{Extracellular hydrolytic enzymes}

Extracellular enzymes secreted by Candida albicans are claimed to be virulence factors responsible for penetration of the yeast into host cells [527]. The secreted aspartyl proteinases (SAP) and phospholipases $(P L)$ are two rather large families of C. albicans enzymes, some of which have been associated with virulence [53]. Secreted aspartic proteases (Saps) contribute to the virulence of $C$. albicans, a major fungal pathogen of humans [97]. The differential expression of specific 
virulence factors at different stages of an infection could be the reason why $C$. albicans not only has single genes for extracellular hydrolytic enzymes, but gene families. Both secreted aspartate proteinases (Saps) and secreted lipases (Lips) from $C$. albicans are encoded by at least 10 different genes. This high number of similar genes might provide it $C$. albicans with the ability to secrete a specific and appropriate enzymatic response at distinct stages of an infection [447].

\section{Phenotypic switching}

The basic mechanism of phenotypic switching is not clear, although is known that C. albicans can switch among different phenotypes (smooth, rough, star, stippled, hat, irregular wrinkle and fuzzy) at high frequency [430,435-438]. Of all the switch phenotypes described, the most studied is the white opaque system in strain WO-1, in which smooth, white colonies, switch to flat and grey colonies (opaque) (Figure 1.4). The role of phenotypic switching in the virulence of this organism remains to be elucidated, although it has been observed that opaque cells colonize the skin in a cutaneous model more than white-phase cells, but are less virulent in a systemic animal model $[257,437]$.

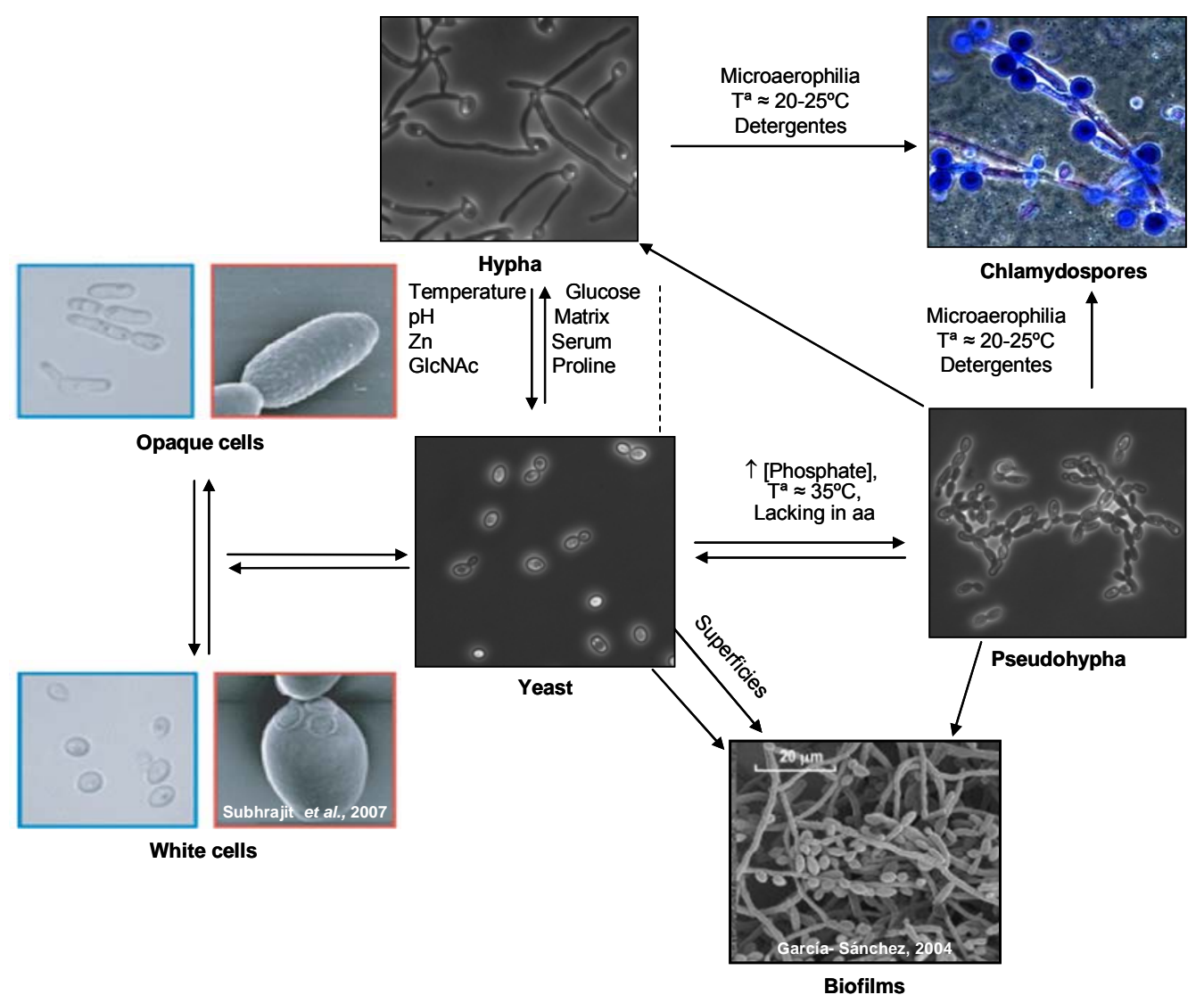

Figure 1.4 Morphological forms of $C$. albicans 


\section{Biofilm formation}

The ability to adhere to surfaces and develop as a multicellular community is an adaptation used by most microorganisms to survive in changing environments. Biofilm formation proceeds through distinct developmental phases and impacts not only medicine but also industry and evolution. In organisms such as the opportunistic pathogen $C$. albicans, the ability to grow as biofilms is also an important mechanism for persistence, facilitating its growth on different tissues and a broad range of abiotic surfaces used in medical devices [334]. Biofilm formation is a major virulence attribute of Candida pathogenicity which contributes to higher antifungal resistance [413]. Candida species, particularly C. albicans, readily form biofilms, consortia of cells that coexist as an organized community with a complex three-dimensional architecture that is enveloped within an exopolysaccharide matrix, attached to a solid substratum [82,83,109]. Mature biofilms are characterized by the production of a thick extracellular matrix and an altered resistance phenotype to common antifungal agents $[82,83]$. The basis of the characteristic drug resistance of cells growing in biofilms is a source of much speculation, although recent studies suggest multiple factors involved in this process $[19,409]$.

\section{Hyphal-specific gene transcription}

A variety of factors, including temperature, amino acids, $\mathrm{pH}$ changes and serum can induce the yeast-hypha transition in C. albicans [121,158,312]. The contribution of a range of signalling pathways to morphogenesis has been investigated, suggesting that the yeast-hypha transition is the subject of both positive and negative regulation [158,500]. Several genes have been identified whose expression is induced during hyphal induction. Such genes are listed next, and most of them encode either cell-wall proteins or secreted proteins:

- ECE1: expression of this gene was not detected when C. albicans grew as a budding yeast cell but was observed within 30 min after cells had been induced to form hypha [32].

- HYR1: encodes a non-essential cell wall glycoprotein and null mutant for this gene also displays a normal hyphal development [18].

- RBT1 and RBT4: these two genes repressed by TUP1 encode a cell-wall protein 
and a secreted protein, respectively [37].

\section{Regulation of central metabolic pathways in a fungal pathogen}

To grow, a microbe must assimilate carbon. Pathogens such as $C$. albicans, which can thrive within diverse niches such as the skin, mucous membranes, blood and internal organs of its human host and in biofilms [51,358], must display sufficient metabolic flexibility to assimilate the available nutrients in these niches. Data from several transcript profiling studies are consistent with this notion [41]. For example, amino acid biosynthetic genes are up-regulated in $C$. albicans cells growing in yeast-hypha transition (our data in Chapter II). Also, following exposure to human neutrophils or cultured macrophages, C. albicans populations up-regulate amino acid biosynthetic genes and display a shift from fermentative to non-fermentative metabolism $[134,286,393]$. This includes the down-regulation of glycolytic genes and the activation of glyoxylate cycle genes (ICL1 and MLS1), which facilitate the assimilation of two-carbon compounds in concert with gluconeogenic genes (PCK1 and $F B P 1)$. Further evidence for the activation of glyoxylate cycle genes following exposure to macrophages has been obtained by differential display reverse transcription polymerase chain reaction (PCR) $[287,373]$. It has been reported that C. albicans ic/1/icl1 mutant display attenuated virulence in the mouse model of systemic candidiasis. This has led to the suggestion that the glyoxylate cycle is required for fungal virulence $[287,288]$. However in S. cerevisiae, gluconeogenic and glyoxylate cycle genes are exquisitely sensitive to glucose [526]. These genes are repressed by glucose at concentrations as low as $0.01 \%$, which are well below those present in the bloodstream. If $C$. albicans gluconeogenic and glyoxylate cycle genes are regulated in an analogous fashion to those in $S$. cerevisiae, which is often presumed to be the case, one would expect them to be repressed during systemic infections. How then is the glyoxylate cycle is required for the establishment of systemic C. albicans infections? To address this apparent paradox, it has been revisited the role of the glyoxylate cycle and glycolytic and gluconeogenic pathways in $C$. albicans virulence. Most of the enzymes on the glycolytic pathway catalyse reversible reactions that also contribute to gluconeogenesis. However, two steps in glycolysis are essentially irreversible and these are catalysed by the glycolysis-specific enzymes, phosphofructokinase and pyruvate kinase. PFK2 encodes one of two phosphofructokinase subunits, and 
PYK1 encodes pyruvate kinase. PCK1 encodes the gluconeogenenic specific enzyme, phosphoenolpyruvate carboxykinase. ICL1 encodes the glyoxylate cycle enzyme, isocitrate lyase (Figure 1.5). This view arises from both a general metabolism perspective and the identification of the precise metabolic points that distinguish both morphological phases. Some drug targets and genes related to virulence were also detected in several metabolic pathways, and the majority of genes involved in signal transduction pathways possibly participating in cell differentiation and infection are annotated in C. albicans.

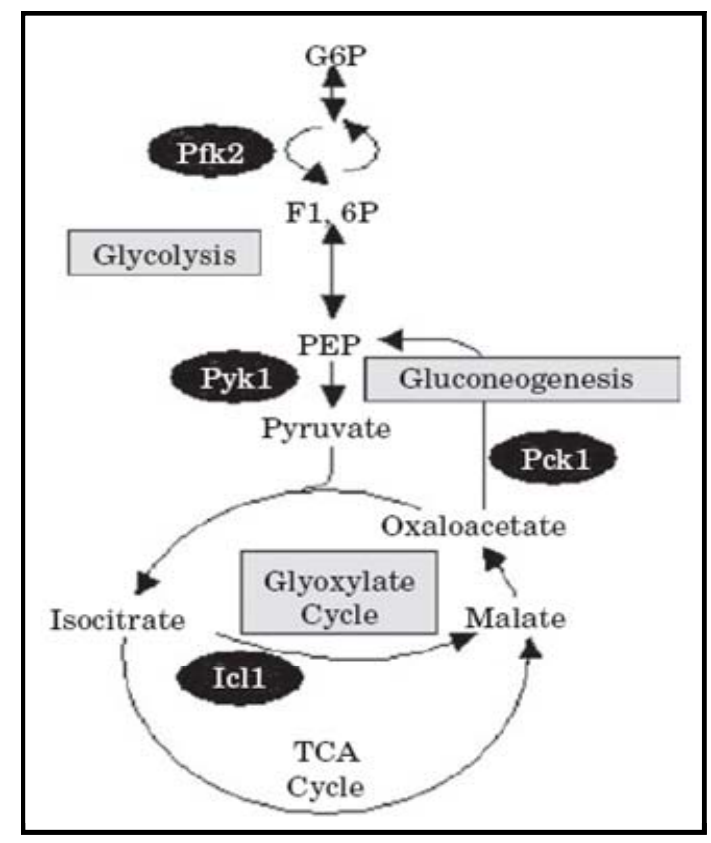

Figure 1.5 Cartoon of central carbon metabolism

\section{Virulence phenotypes and the choice of animal model}

In C. albicans, attenuation of mouse lethality in mutant fungal strains has been the most common approach to elucidating the roles of specific genes in virulence. It is clear, however, that expression of virulence by a fungus can depend on the particular host niche that is being affected. It is therefore possible to miss virulence factors when only one model for virulence is tested. In C. albicans, disruption of the GCN5 (a histone acetyltransferase) gene led to attenuated lethality when injected intra-peritonealy into mice (our data in chapter III), whereas disruption of the related HDA1 (a histone deacetylase encode) gene had no effect on lethality [307]. Similarly, in C. neoformans, a mutant with a disrupted URE1 urease gene showed normal virulence when injected intracisternally into rabbits, but showed attenuated 
lethality in a mouse infected intravenously [77]. Extensive studies of the family of secreted proteinase enzymes (SAP gene products) in C. albicans have also indicated differential expression of the individual SAP genes according to the stage and type of infection under investigation [407]. Clearly, there is no substitute for thorough investigation of virulence phenotypes in as many models of infection as possible in vivo and ex vivo, particularly when single-gene disruptants are under scrutiny, if the true role of virulence molecules in the infection process is to be fully determined.

The actin cytoskeleton is required for the dimorphic switch in Candida albicans

During hyphal development, cell-surface expansion is highly restricted to the apical tip of hyphal filaments. This polarized apical growth requires the actin cytoskeleton. C. albicans yeast cells display a temporal change in the organization of the actin cytoskeleton during cell cycle progression like that in S. cerevisiae cells, whereas the actin cytoskeleton is polarized at the tip of all hyphal cells during filamentation [12]. In S. cerevisiae and other organisms, the small GTPase of the Rho subfamily Cdc42p is known to be critical for establishing a polarized actin cytoskeleton in response to extracellular stimuli. In the filamentous ascomycete Ashbya gossypii, three Rho-GTPases control distinct steps during polarized hyphal growth with Cdc42 $p$ being required for the establishment of actin polarization [499]. C. albicans Cdc42p is also essential for cell morphogenesis [193] as down-regulated in our hypha defect mutant cells, is probably responsible for establishing the polarized actin cytoskeleton during hyphal induction.

\section{Transcriptional control and chromatin remodelling proteins}

The organization of the eukaryotic chromatin has a major impact on all nuclear process involving DNA substrates. Gene expression is affected by the positioning of individual nucleosomes relative to regulatory sequence elements, by the folding of the nucleosomal fiber into high-order-structures and by the compartmentalization of function domains within the nucleus. Because site-specific acetylation of nucleosomal histones influence all three aspects of chromatin organization, it is central to the switch between pemissive and repressive chromatin structure. The targeting of enzymes that modulate the histone acetylation status of chromatin, in 
synergy with the effects mediated by other chromatin remodelling factores, is central to gene regulation.

\section{Chromatin structure and nucleosomes}

Sience the discovery of the basic principles of chromatin organization, which is involves in the wraping of DNA around histone octamers to form nucleosomes and the folding of the nucleosomal fiber in to higher-order-structures, the question of how such extensive packaging can be compatible with reactions that be involve 'reading' the DNA has stimulated extensive reaserch. The fundamental unit of chromatin is the nucleosome, which consists of 147 base pairs of DNA wrapped 1.6 times around a core of eight histones (two molecules of $\mathrm{H} 2 \mathrm{~A}, \mathrm{H} 2 \mathrm{~B}, \mathrm{H} 3$ and $\mathrm{H} 4$ ) (Figure 1.6 A, B) $[284,290]$. Chromatin structure is central for the regulation of gene expression [471], and defined as a complex of DNA, histones, and non-histone proteins from which eukaryotic chromosomes are formed.

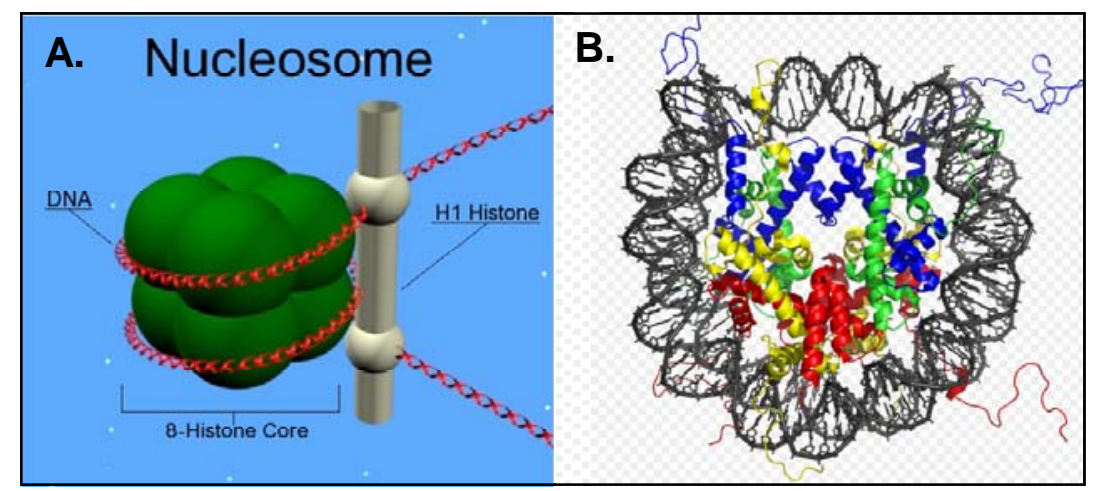

Figure 1.6 (A) Nucleosome structure. (B) The crystal structure of the nucleosome core particle consisting of $\mathrm{H} 2 \mathrm{~A}, \mathrm{H} 2 \mathrm{~B}, \mathrm{H} 3$ and $\mathrm{H} 4$

The core histones are small proteins with a basic charge that share the same basic structure: a globular C-terminal domain critical to nucleosome formation and a flexible N-terminal tail that protrudes from the nucleosome core [404].

It soon became apparent many aspects of chromatin structure could be explained by interaction between nuclesomal histones and DNA, neighboring nucleosomes and non-histone proteins. Most of these interactions involve the $\mathrm{N}$-terminal tails of the core histones, which reach out from the rather compact nuclesomal core particle. The flexible $\mathrm{N}$-terminal tails are enriched with basic residues which are theorized to interact with the negatively charged backbone of DNA [10]. The four canonical histone proteins are composed of a structured central (globular) domain 
that is in close contact with the DNA and a less well-structured amino-terminal tail domain. Histones are also distinguished by the fact that they undergo abundant post-translational modifications to both the globular and tail domains. In the presence of linker histones or heterochromatin associated-proteins, nucleosomes can be condensed into $30 \mathrm{~nm}$ diameter fibers and high-ordered assemblies whose structures are poorly understood. These hierarchical structures form a repressive environment that inhibits the activities of enzymes that require direct access to the DNA template, such as in transcription, DNA repair, replication and recombination. Chromatin condensation can be modulated through a variety of mechanisms, including covalent modifications of DNA and histones. While DNA undergoes methylation of its cytosine bases within $\mathrm{CpG}$ repeats, histones are subject to a myriad of modifications in their random coil $\mathrm{N}$-terminal tails, and to a lesser extent within their C-terminal tails and globular domains [245]. The 1960s and 1970s witnessed the discovery of a multitude of histone modifications including lysine acetylation [9], lysine methylation [9,335], serine phosphorylation [238], ADP ribosylation [352] and ubiquitination [152,214]. Although data from many laboratories furnished circumstantial evidence linking modification states to the transcriptional status of genes, the exact biological functions of histone modifications remained enigmatic for several decades.

In 1996, two groups reported the discovery of histone modifying enzymes that were related by sequence homology to previously identified transcriptional regulators in S. cerevisiae. Utilizing an affinity matrix, Schreiber et al $[305,411]$ isolated a mammalian histone deacetylase (HDAC) that harbors $60 \%$ sequence identity with the yeast transcriptional repressor Rpd3p [462]. Concurrently, Allis et al [11] purified a histone acetyltransferase (HAT) from Tetrahymena thermophila that is highly homologous to the yeast transcriptional adaptor Gcn5p [45]. The identification of these enzymes represented a milestone in understanding the biological functions underlying histone modifications because they furnished the first direct evidence unequivocally linking histone modification states and transcriptional regulation. The discovery of Gcn5p and Rpd3p presaged the identification and characterization of other families of HATs and HDACs as well as other classes of histone modifying enzymes, including kinases [405,469], lysine and arginine-specific methyltransferases [89,380], arginine deiminases [78,496], ubiquitinases [387], deubiquitinases [119,187], and lysine- and arginine-specific 
demethylases (HDMs) [84,473]. Some studies had implicated many of these proteins in transcription regulation or other genomic functions, further underscoring the correlation between histone modifications and chromatin-dependent processes. Collectively, these efforts have transformed our understanding of the role of chromatin modifications in governing eukaryotic gene expression and other DNAdependent functions. Following their discovery, structural and biochemical studies have played a central role in defining the molecular basis for the substrate specificities and catalytic mechanisms of histone modifying enzymes. This paragraph of chapter summarizes current advances in understanding these enzymes, focusing on mechanisms of HATs (GCN5) and HDACs (HDA1).

\section{Histone acetylation and transcriptional control}

Activation of transcription in eukaryotes often requires modification of the chromatin template. Among the well-characterized chromatin-modifying enzymes, histone acetyltransferases (HATs) are known to regulate gene expression by adding acetyl groups to lysine residues within the $\mathrm{N}$-terminal tails of histones [392].

Transcriptional activators often require histone acetyltransferases (HATs) for full activity. The common explanation is that activators directly recruit HATs to gene promoters to locally hyperacetylate histones and thereby facilitate transcription complex formation [216]. A number of HAT enzymes have been isolated from various organisms [57]. The identification of new HATs has resulted in other important findings. It has been demonstrated that HATs are evolutionarily conserved from yeast to humans, that HATs generally contain multiple subunits [236], and that the functions of the catalytic subunit depend largely on the context of the other subunits in those complex [480]. Furthermore, recent work on HAT complex has resulted in their categorization on the basis of their catalytic domains. The picture is complicated by the observation that some HAT enzymes can modify different histone substrates and that some HAT enzymes also acetylate an ever growing number of non-histone substrates [523]. In addition, histone acetylation is a dynamic reversible process. The balance of histone acetylation is important for proper cellular function and the cell has evolved enzymes that catalyse the removal of acetyl groups, termed histone deacetylases (HDACs) [118]. HATs are a diverse set of enzymes; the multiprotein complexes in which they reside also vary. Different HAT complex are composed of various unique subunits. The combinations of these 
subunits contribute to the unique features of each HAT complex. For example, some subunits have domains that cooperate to recruit the HAT to the appropriate location in the genome; these include bromodomains, chromodomains, WD40 repeats, Tudor domains and PHD fingers. It has been discussed various histone substrates of HAT complex and the various HAT subunits, and how protein domains read and interpret the 'histone code' that is, the combination of posttranslational marks on the histones [269].

\section{HATs serve a multiplicity of functions}

As mentioned a number of different HAT complex exist in yeast and other organisms, and these HAT complex are composed of assorted proteins with various chromatin-binding domains that influence HAT recruitment. What is the reason for such complexity? In large part, the answer lies in the fact that HATs serve a multiplicity of functions. First, HAT complex modify histones in relation to the state and/or function of the DNA that the histones are compacting. As discussed above, HAT enzymes take advantage of their associated proteins as well as cellular signals for their recruitment to distinct regions of the genome. In turn, these associations allow them to carry out specialized functions, which the enzyme would not be capable of on its own $[256,452]$. Such specialized functions of HATs include roles in processes such as genome stability and DNA repair. Second, HAT enzymes do not only modify histones, but function to acetylate an increasing number of non-histone substrates [523]. For example, many HATs also function as transcription factor acetyltransferases (FATs), which means that these same enzymes can acetylate nonhistone substrates to regulate their activities in the cell [244,523]. Recently, much research has gone into deciphering specific functions of HATs and the mechanisms by which they carry out these functions. As is the case with the function of many proteins, there are a number of factors that can influence the manner in which they function. The same is true for HATs. HATs carry out a broad range of functions and the regulation of these functions is in many cases vital for maintaining the integrity of the organism.

\section{Histone acetyltransferases GCN5}

The yeast transcriptional coactivator GCN5, a histone acetyltransferase (HAT), is part of a large multimeric complex that is required for chromatin remodeling and 
transcriptional activation [125]. In the yeast $S$. cerevisiae, one of the best known HATs is Gcn5p, which is the catalytic subunit of the SAGA complex that acetylates primarily histones $\mathrm{H} 3$ and $\mathrm{H} 2 \mathrm{~B}$ [453,535]. Work in recent years has provided overwhelming evidence that this HAT-containing complex functions as a coactivator and directly participates in the transcription process by being specifically recruited by activators to the promoter of many genes [392]. Recent genome-wide location studies suggest that Gcn5-containing complex may actually be recruited by regulatory proteins to the upstream activating sequence (UAS) of most active genes [385].

In budding yeast the histone acetyltransferase Gcn5p is involved in cell cycle progression, whereas its absence induces several mitotic defects, including inefficient nuclear division, chromosome loss, delayed G2 progression, and spindle elongation. The fidelity of chromosome segregation is finely regulated by the close interplay between the centromere and the kinetochore, a protein complex hierarchically assembled in the centromeric DNA region, while disruption of GCN5 in mutants of inner components results in sick phenotype. In these synthetic interactions involving the ADA complex lay the genetic basis for the critical role of Gcn5p in kinetochore assembly and function. Gcn5p have been physically linked to the centromere, where it affects the structure of the variant centromeric nucleosome [488]. Gcn5p, a major HAT in yeast, has diminished histone acetyltransferase activity in particular mutants, providing a plausible explanation for reduction of cellular acetylation levels in vivo.

\section{C. albicans histone acetyltransferase GCN5}

The yeast transcriptional coactivator GCN5 is part of large multimeric complex that are required for chromatin remodeling and transcriptional activation. Like other eukaryotes, the $C$. albicans DNA is organized into nucleosomes and the genome encodes components of chromatin-remodeling complex. GCN5 is conserved in Candida species and that the most homologous regions are within the HAT domain and the bromodomain (Figure 1.7 and 1.8).

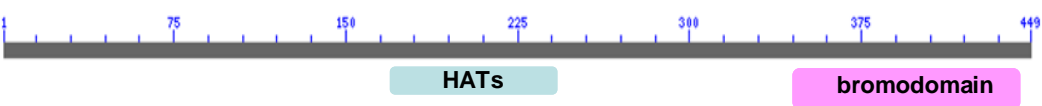

Figure 1.7 Diagram representing the Hat and Bromodomain of C. albicans GCN5 
Many yeast genes encode histone acetytransferase and histone deacetylase proteins in $C$. albicans and their relative phenotype are totally unknown. Thus, our laboratory have performed a phenotypic analysis of yeast strain carrying deletions of GCN5 and other genes encoding members of the HATs and HDACs family of $C$. albicans. The $C$. albicans genome contains six ORFs that potentially encode histone deacetylases proteins containing the HDACs domine and five histone acetyltransferases. A search of the NCBI database (http://www.ncbi.nlm.nih.gov/BLAST) indicates that a Candida albicans protein named Gcn5 (orf19.705) shares the highest similarity with Pichia guilliermondii ATCC 6260 (81\%), Pichia stipitis (78\%), Lodderomyces elongisporus (70\%), Debaryomyces hansenii (73\%) and Saccharomyces cerevisiae (70\%)(Figure 1.8). The GeneBank accession number for the $C$. albicans GCN5 nucleotide sequence is NW_139602.

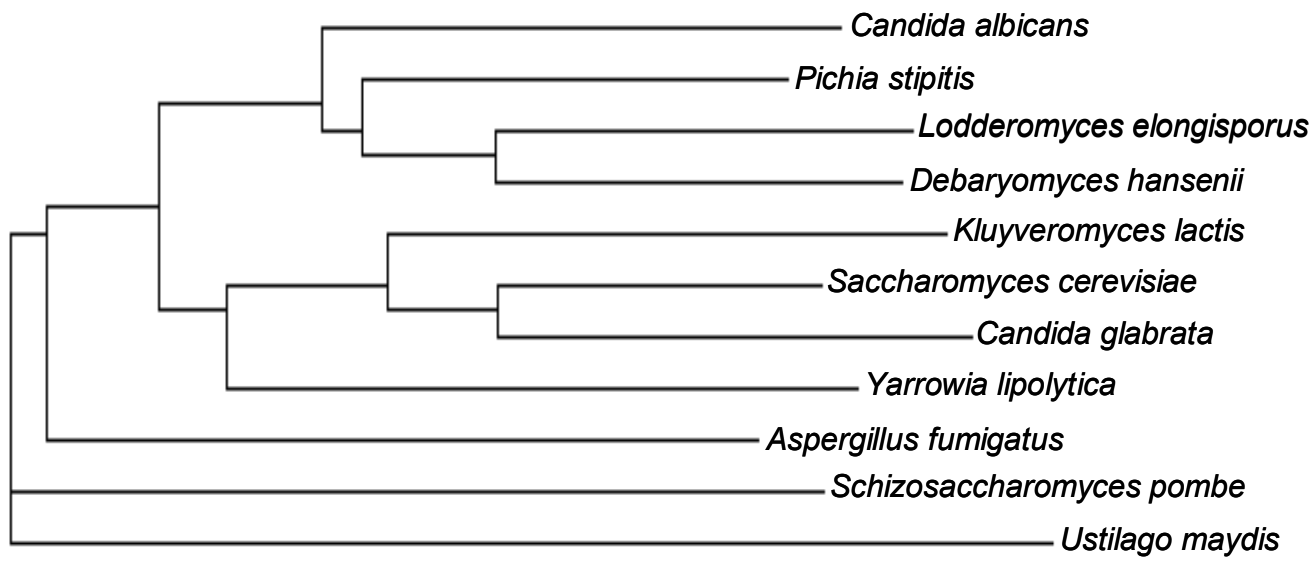

Figure 1.8 Dendograms representing the phylogenetic relationships of $C$. albicans GCN5

Yeast Gcn5p, the ancestor of the histone acetyltransferase (HAT) family, marks histone $\mathrm{H} 3$ and $\mathrm{H} 4$ tails with an $\varepsilon$-acetyl group on specific lysines [392,516]. Gcn5p does not act on its own but rather as the catalytic subunit of two separate and conserved HAT complexes named ADA and SAGA (Spt-Ada-Gcn5acetyltransferase). Specifically, Spt proteins are exclusively present in SAGA, while Spt20p is necessary for maintaining the integrity and function of the whole complex [517]. Histone acetylation by Gcn5p is implicated in the displacement of nucleosomes from promoters during transcriptional activation and also in aiding the recruitment of TATA binding protein, RNA polymerase II and coactivators [154]. Acetylation therefore facilitates the formation of an accessible "open" chromatin structure corresponding to the transcribing genome $[99,251,449]$. During cell 
division, chromatin remodeling expands to wide chromosomal regions, producing long waves of compaction and decondensation over the whole genome at each cell division [93,489]. Disturbing the HAT/histone deacetylase balance therefore alters protein activities on a cellular scale, which leads to various diseases, including cancer. Several reports have highlighted the involvement of Gcn5p alone or in combination with other HATs such as Sas3p in the cell cycle $[199,223]$ and in the transcriptional regulation of a set of genes required at the end of telophase [247]. In mammalian cells, loss of the homologue Gcn512p is lethal during embryogenesis, induces a high level of apoptosis [519], and affects G2/M transition in null mouse embryonic stem cells [277]. In yeast, the specialized centromeric nucleosome, which contains the histone H3 variant Cse $4 p$ [318], is necessary for the assembly and interactions of inner kinetochore components at the centromere [72] and for the correct attachment of the chromosomes to the spindle in metaphase. Mutations in the centromeric/kinetochore components or epigenetic modifications of this structure may lead to chromosome missegregation and G2/M delay. In yeast Gcn5p is, in fact, physically linked to the centromere, where it affects the structure of the variant centromeric nucleosome. It offers a key insight into a Gcn5pdependent epigenetic regulation at centromere/kinetochore in mitosis [488].

\section{HAT's and their biological functions}

In eukaryotes, chromatin reorganization is essential for transcription, and histone acetylation has been directly implicated in this process owing to the discovery that transcriptional activators associate with histone acetyltransferases (HATs) and transcriptional repressors recruit histone deacetylases. Although the precise molecular underpinnings are still being defined, recent evidence suggests that histone acetyltransferases also play important roles in histone-modulated processes such as DNA replication, recombination and repair. In addition, acetylation of transcriptional cofactors and other proteins is an efficient means of regulating a diverse range of molecular interactions $[90,448]$. Moreover, acetylation (Ac) of histones has been widely demonstrated as an important post-translational modification that can regulate many DNA-based processes including gene expression [252,449]. There are numerous lysines $(K)$ in both the amino termini and the globular domains of the core histones including $\mathrm{K}_{9}, \mathrm{~K}_{14}, \mathrm{~K}_{18}, \mathrm{~K}_{23}, \mathrm{~K}_{27}$ and $\mathrm{K}_{56}$ in histone $\mathrm{H} 3$ and $\mathrm{K}_{5}, \mathrm{~K}_{8}, \mathrm{~K}_{12}$ and $\mathrm{K}_{16}$ in $\mathrm{H} 4$ as well as other lysines in histones 
$\mathrm{H} 2 \mathrm{~A}$ and $\mathrm{H} 2 \mathrm{~B}$. These lysines can be acetylated or deacetylated by various histone acetyltransferases (HATs) or deacetylases (HDACs) that show specificity toward histone subtypes as well as individual residues within a given histone. For instance, Gcn5p, a member of the Spt-Ada-Gcn5-acetyltransferase (SAGA) complex in $S$. cerevisiae, is the main HAT for lysine residues in the amino terminus of histone $[160,161,251]$ whereas Rtt109p specifically acetylates $K_{56}$, a lysine in the globular domain of H3 [174]. Moreover, it is apparent that other DNA-based processes such as replication, recombination and repair require chromatin remodeling and that histone acetylation may play a key role. HAT's can be classified with respect to their intracellular location and substrate specificity as either nuclear A-type (HAT A) or cytoplasmic B-type (HAT B). A-type HAT's are involved in the post-synthetic acetylation of all four nucleosomal core histones and have long been thought to promote transcription related acetylation although their involvement in other processes such as DNA repair and replication is also likely [249]. Conversely Btype HAT's are believed to have a housekeeping role in the cell, acetylating newly synthesised free histones (primarily histone $\mathrm{H} 4$ at lysines 5 and 12) in the cytoplasm for transport into the nucleus, where they may be deacetylated and incorporated into chromatin $[11,241,394]$. It is important to note that HATs function in several other processes that are becoming clear, including DNA replication and recombination. The study of HATs is a broad and complex field that is still expanding, and although our knowledge of these important proteins and complex has increased substantially in recent years there is still much to learn. In time, acetylation and subsequent deacetylation of proteins might be looked at in a similar fashion as phosphorylation and dephosphorylation in regulating cellular processes.

\section{Histone deacetylases}

Histone tails are normally positively charged due to amine groups present on their lysine and arginine amino acids. These positive charges help the histone tails to interact with and bind to the negatively charged phosphate groups on the DNA backbone. Chromatin acetylation correlates with transcriptional activity (euchromatin), whereas deacetylation correlates with gene silencing. HDACs are also involved in the reversible acetylation of non-histone proteins. Yeast cells contain a group of related HDACs that include Rpd3p, Hda1p, Hos1p, Hos $2 p$ and Hos3p $[396,462]$. The earliest example of the targeting of HDACs to a regulatory 
sequence was that of the Rpd3p, which is a member of the class I HDACs (the mammalian orthologues of which include HDAC1), HDAC2 and HDAC3. In yeast, Rpd3p belongs to a large multiprotein complex that includes Sin3p, Sap30p [537], Sds3p [33,98,266], Pho23p [283] and Ume1p [253], and several other uncharacterized factors [144]. Yeast Hda1p (which is a member of the class II HDACs) is related to HDAC4, HDAC5 and HDAC6 in mammals, and is recruited to its target promoters through the Tup1p repressor [515]. Similar to Rpd3p targeting, recruitment of the Hda1p complex results in local deacetylation in a region spanning about 2 nucleosomes that are adjacent and downstream of its recruitment site. However, unlike the broad substrate specificity of Rpd3p, the Hda1p complex only deacetylates Lys residues in histones $\mathrm{H} 3$ and $\mathrm{H} 2 \mathrm{~B}$ (but not the $\mathrm{H} 4$ or $\mathrm{H} 2 \mathrm{~A}$ ) amino-terminal tails [515]. The acetyltransferase Esa1p, which is the catalytic component of the NuA4p (for nucleosome acetyltransferase of histone H4) multiprotein complex, acetylates $\mathrm{H} 4$ and $\mathrm{H} 2 \mathrm{~A}$ histones $[8,453]$ and seems to be targeted to promoters of the ribosomal protein genes in yeast [381]. The functional roles of these various substrate specificities for HDACs and HATs are as yet unknown. Although acetylation microarray data for HATs have not been reported so far, the genome-wide expression analyses of deletion mutants (GCN5 and HDA1 as simple and double mutant) of these enzymes in $C$. albicans have provided some evidence for a division of labour among HATs and HDACs.

\section{Crosstalk between histone acetylation/deacetylation and chromatin remodeling proteins}

It has been shown that ATP-dependent chromatin remodeling factors cooperate with histone acetyltransferases (HATs) and histone deacetylases (HDACs) in gene regulation. The functional link between ATP-dependent remodeling and histone acetylation was first suggested from genetic studies in yeast [369]. Mutations in Gcn5p of the SAGA complex in combination with mutations in the SWI/SNF complex resulted in lethal phenotypes, indicating a concerted interaction between components of these two complexes. It is suggested that SWI/SNF-dependent remodeling occurs prior to Gcn5-dependent histone acetylation [248], and is required for acetylation to occur. This observation implies that histone acetyltransferase complex cannot penetrate the compact chromatin without remodeling. Alternatively, histone acetylation may occur followed by ATP- 
dependent remodeling, thereby altering chromatin structure for general transcription factor binding [1], or providing a better surface for stabilization of ATPdependent remodeling complex [179]. Isw2p is an ISWI family ATP-dependent remodeling complex in yeast. Although Isw2p and $\operatorname{Sin} 3 p-R p d 3 p$ histone deacetylase complex have unique biochemical activities, genetic studies indicate that Isw2p and $\operatorname{Sin} 3 p-R p d 3 p$ function synergistically to regulate gene repression [126]. Both nucleosome remodeling ATPase Mi-2 and histone deacetylases (HDAC1/2 in mammals and RPD3 in $X$. laevis) reside in the NURD (nucleosome remodeling and deacetylation) complex, implying that histone modification (deacetylation) and ATP-dependent remodeling are synergistic actions in chromatin remodelling $[172,240]$. The ATPase activity of Mi-2 was shown to be able to increase the efficiency of histone deacetylation by NURD complex [172].

\section{cDNA microarray analysis of differential gene expression in C. albicans}

The near completion of sequencing the $C$. albicans genome has made it possible to employ genomic technologies, such as microarray analysis, to aid in identifying key genes involved in such clinical problems as the acquisition of high-level resistance to azole antifungal agents. In addition, DNA microarrays provide a snapshot of an organism's genome in action by revealing the relative transcript levels of thousands of genes at a time [281]. Microarray analysis gives researchers the ability to identify genes involved in processes such as acquisition of azole resistance and to use the data in a way that may lead to clinical approaches to inactivate these genes and improve patient outcomes. Differences in gene expression underlie many of the phenotypic variations in $C$. albicans, yet approaches to characterize such differences on a genome-wide scale are well developed by using cDNA microarray techniques. Phenotypic diversity can often be traced to the differential expression of specific regulatory genes [56,153]. Recently, microarray experiments revealed large-scale differences in the genome-wide transcription response of related organisms to equivalent environmental conditions. For example, the transcription program underlying insect metamorphosis differs considerably between related species of the Drosophila melanogaster subgroup [384]. Similarly, both the meiotic and the mitotic cell cycle transcription program have diverged significantly between the budding and the fission yeasts [397]. The impact of such large-scale variations in gene expression on the phenotypes of the organisms is not yet understood. 
Existing computational approaches for the comparative analysis of microarray gene expression data have focused primarily on evolutionarily distant model organisms, for which large sets of expression data are available $[7,315]$. Such studies demonstrated that conservation of co-expression can improve functional gene annotation $[30,450]$. Common expression programs are invoked by related perturbations, such as adult onset in the nematode Caenorhabditis elegans, and the fruit fly D. melanogaster [315]. 
Table 1.1 Some of example about cDNA microarray analysis of differential gene expression in C. albicans

\section{Analysis/publication Drug resistance} cDNA Array Analysis of the Differential Expression Change in Virulence-related Genes During the Development of Resistance in Candida albicans [520]

Genome-wide expression profiling of the response to ciclopirox olamine in Candida albicans [272]

A gain-of-function mutation in the transcription factor Upc2p causes up-regulation of ergosterol biosynthesis genes and increased fluconazole resistance in a clinical Candida albicans isolate.[114]

A genome-wide steroid response study of the major human fungal pathogen Candida albicans [21]

The transcription factor Mrr1p controls expression of the MDR1 efflux pump and mediates multidrug resistance in Candida albicans [326]

\section{Yeast-hypha transition}

The Flo8 transcription factor is essential for hyphal development and virulence in Candida albicans [54]

In vivo and ex vivo comparative transcriptional profiling of invasive and non-invasive Candida albicans isolates identifies genes associated with tissue invasion [466]

\section{Abstract of result}

In this work, 16 virulence-related genes were identified, whose differential expressions were newly found to be associated with the resistant phenotype. Furthermore, the up-regulation of EFG1, CPH2, TEC1, CDC24, SAP10, ALS9, SNF1, SPO72 and BDF1, and the down-regulation of $R A D 32$, IPF3636 and UB/4 resulted in stronger virulence and invasiveness in the resistant isolates compared with susceptible ones. These findings provide a link between the expression of virulence genes and development of resistance during $C$. albicans infection in bone marrow transplant (BMT) patients, where induces hyphal formation and expression change in multiple virulence factors

By cDNA microarray method, 49 genes were found to be responsive to ciclopirox olamine, including 36 up-regulated genes and 13 down-regulated genes. These included genes involved in small molecule transport (HGT11, HXT5, ENA22, PHO84 and CDR4), iron uptake (FRE30, FET34, FTR1, FTR2, SIT1) and cell stress (SOD1, SOD22, CDR1, DDR48). Mutants disrupted for $C D R 2$ and both $C D R 1$ and $C D R 2$, as well as a clinical isolate overexpressing $C D R 1$ and $C D R 2$, showed no change in susceptibility to ciclopirox olamine compared with the respective parent.

By comparing the gene expression profiles of the fluconazole-resistant isolate and of strains carrying wild-type and mutated UPC2 alleles, they identified target genes that are controlled by Upc2p. They show for the first time that a gain-of-function mutation in UPC2 leads to the increased expression of ERG11 and imparts resistance to fluconazole in clinical isolates of $C$. albicans.

In the absence of steroid receptors and any known mechanism of gene regulation by steroid hormones in Candida albicans. They did a genomewide analysis of $C$. albicans cells treated with progesterone using cDNA microarrays to find the complete repertoire of steroid responsive genes. A total of 99 genes were found to be significantly regulated by progesterone, among them 60 were up-regulated and 39 were down-regulated. Several genes associated with hyphal induction and the establishment of pathogenesis was also found up-regulated. In silico search for various transcription factor (TF) binding sites in the promoter of the affected genes revealed that EFG1, CPH1, NRG1, TUP1, MIG1 and AP-1 regulated genes are responsive to progesterone.

By comparing the transcriptional profiles of drug-resistant $C$. albicans isolates and mrr1s mutants derived from them and of $C$. albicans strains carrying wild-type and mutated MRR1 alleles, they defined the target genes that are controlled by Mrr1p. Many of the Mrr1p target genes encode oxidoreductases, whose up-regulation in fluconazole-resistant isolates may help to prevent cell damage resulting from the generation of toxic molecules in the presence of fluconazole and thereby contribute to drug resistance. The identification of MRR1 as the central regulator of the MDR1 efflux pump and the elucidation of the mutations that have occurred in fluconazoleresistant, clinical $C$. albicans isolates and result in constitutive activity of this trancription factor provide detailed insights into the molecular basis of multidrug resistance in this important human fungal pathogen.

Genome-wide transcription profiling of efg1/efg 1 and flo8/flo8 using a $C$. albicans DNA microarray suggests that Flo8 controls subsets of Efg1regulated genes. Most of these genes are hypha specific, including HGC1 and IHD1.

By analysing the different phases of intraperitoneal infection from attachment to tissue penetration in a time-course experiment and by comparing the profiles of an invasive with those of a non-invasive strain, they identified genes and transcriptional pattern which are associated with the invasion process. This includes genes involved in metabolism, stress, and nutrient uptake, as well as transcriptional programmes regulating morphology and environmental sensing. 
A generalization of the singular value decomposition approach that is applicable for such a comparative study was applied to cell cycle datasets from $S$. cerevisiae and human [7]. Yet, the challenge of systematically comparing the gene expression program in related organisms is only starting to be addressed.

\section{Polyamines}

Although simple in structure, the polycationic polyamines spermidine and spermine and their diamine precursor putrescine are essential factors for growth in eukaryotic cells [146,313] (Figure 1.9). The total intracellular concentration of the polyamines is in the millimolar range; however, free polyamine concentrations are considerably lower, as they are mostly ionically bound to various anions in the cell including DNA, RNA, proteins and phospholipids. The original discovery of spermine dates back to Leuwenhoek in 1678 , however the tools necessary to study the specific molecular functions in which the polyamines are intimately involved have only recently become available. Understanding the molecular functions of polyamines is complicated by the fact that most of the critical interactions in which the polyamines are involved in readily reversible ionic interactions. Among the roles that polyamines have in the support of cell growth are association with nucleic acids, maintenance of chromatin conformation, regulation of specific gene expression, ion-channel regulation, maintenance of membrane stability, provision of a precursor in the synthesis of eukaryotic translation initiation factor $5 \mathrm{~A}$ (IF5A), and free-radical scavenging $[137,363]$. Several recent studies have been instrumental in furthering our understanding of the molecular functions of polyamines. Polyamines and polyamine metabolism could be used as targets for antiproliferative therapy. This fact is based on several observations suggest that cells synthesize more polyamines when induced to grow; and that the polyamine biosynthetic enzymes are co-ordinately regulated with growth controls. Polyamine metabolism is frequently dysregulated in cancers. Data indicating that the polyamine pathway is a downstream target for known oncogenes and that inhibition of polyamine synthesis disrupts the action of those genes have been published [63,349]. The fact that polyamines appear to be a site of intervention that is distal and common to a number of validated targets makes this target even more intriguing, and validates the active pursuit of agents that interfere with both polyamine metabolism and function as a strategy for antiproliferative intervention. 


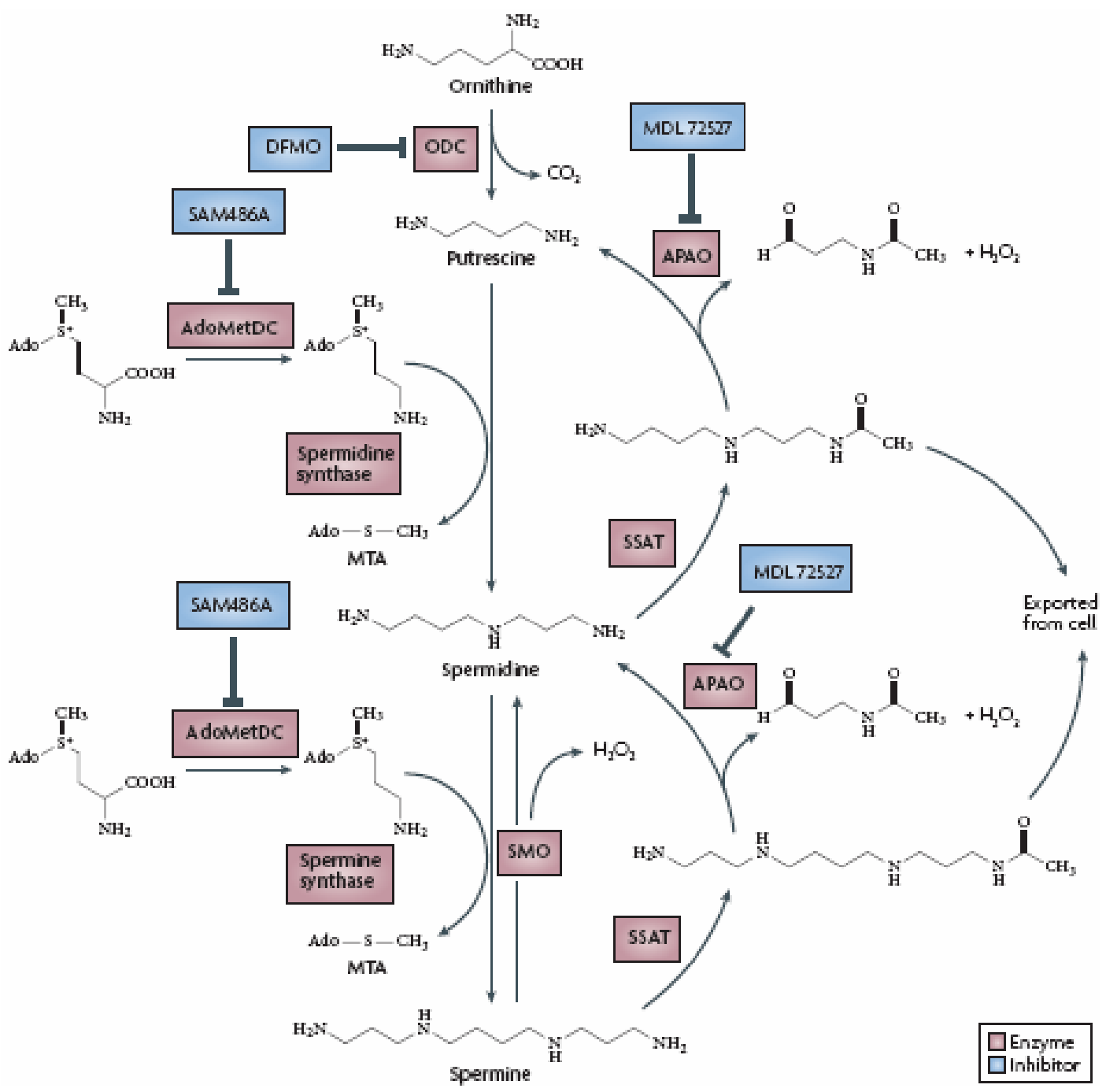

Figure 1.9 Pathway of polyamine synthesis in eukaryotic organisms SAM, S-adenosylmethionine; SAMdc and decarboxylated S-adenosylmethionine, difluoromethylornithine (DFMO), $N, \quad N$ '-bis(2,3-butadienyl)-1,4-butanediamine (MDL-72,527), N1-acetylpolyamine oxidase (APAO), spermidine/spermine N1-acetyltransferanse (SSAT), mammalian spermine oxidase (SMO), S-adenosylmethionine (SAM486A), S-Adenosylmethionine decarboxylase (AdoMetDC).

Although polyamines are abundant and ubiquitous components of all cells, relatively little is known about their function. In mammalian cells, there is a positive correlation between rapid growth and high levels of polyamines [14,319]. Furthermore, addition of growth factors to quiescent cells leads to a rapid induction of polyamine synthesis. The biosynthesis of the naturally occurring polyamines, spermidine and spermine, is tightly regulated by a variety of mechanisms $[58,167,278,325,367,418]$. Fine control of intracellular polyamine levels is necessary, because overproduction of these compounds can lead to cellular toxicity and oncogenic transformation [325]. In C. albicans, polyamine depletion is lethal and suboptimal concentrations inhibit the yeast-hypha transition [188]. 


\section{Polyamine metabolism}

The first rate-limiting step in polyamine biosynthesis is the production of putrescine by the pyridoxal, phosphate-dependent decarboxylase ornithine decarboxylase (ODC). The requirement for ODC activity (and putrescine production) in cell proliferation has been demonstrated in most eukaryots. $O D C$ expression is tightly regulated at several steps from transcription to post-translational modification [365]. ODC is active as a homodimer with a half-life that is among the shortest of any known protein [490] (10-30 minutes in mammalian systems), and, like many shortlived proteins, ODC is destroyed by the $26 \mathrm{~S}$ proteosome. However, unlike most proteins, ODC is not first ubiquitinated, instead a specialized regulatory protein, ODC antizyme [181], binds to the carboxylic end of the monomeric form of ODC and presents the ODC to the $26 \mathrm{~S}$ proteasome for destruction [67,533]. ODC transcription is another important regulatory step in its expression and ODC was the first transcription target of the MYC oncogene to be reported [362], which demonstrates one link between two crucial growth-promoting pathways. It is important to note that spermine is primarily a polyamine found in eukaryotic cells, as prokaryotes do not have a spermine synthase homologue.

\section{Ornithine decarboxylase (ODC)}

Ornithine decarboxylase (ODC) catalyzes the conversion of ornithine into putrescine, the first step in the universal mechanism of polyamine biosynthesis (Figure 1.9), and is one of the most highly regulated enzymes of eukaryotic cells $[69,101,206,219]$. This control is essential, since polyamine excess is toxic and induces apoptosis $[361,371]$. The most important control mechanism of $O D C$ in eukaryotic organisms operates at the post-translational level, regulating the amounts of the enzyme by a unique mechanism of proteasome-dependent degradation $[180,186]$. Originally described in higher eukaryotes, the mechanism has been further described in a few fungal species [87]. This is the first and ratelimiting reaction in the biosynthesis of polyamines in cells [365]. Even modest reductions in mammalian ODC activity can lead to marked resistance to tumor development [348,365]. ODC is turned over rapidly, and this, in part, is because of it being targeted to the $26 \mathrm{~S}$ proteasome without ubiquitination by the protein antizyme, its key regulator [333,532]. Antizyme itself is negatively regulated by 
antizyme inhibitor, a homolog of ODC with a higher affinity for antizyme that has lost the ability to decarboxylate ornithine $[5,332,433,458]$. In addition to its posttranslational regulation, ODC is under transcriptional and translational layers of control. Intracellular levels of polyamines are regulated at various steps, including synthesis, degradation, uptake, and excretion, and cells have developed intricate mechanisms to ensure tight regulation of intracellular polyamine pools [492].

\section{Function of polyamines in eukaryotic cells}

The function of polyamines in mammalian cells may be much more complex, in view of their implicated roles in the regulation of diverse cellular activities such as transcriptional, translational, and posttranslational levels affecting proliferation, transformation, differentiation, and apoptosis. Indeed, by usinng inhibitors [351], have demonstrated an independent role for polyamines and elF5A in supporting the proliferation of mouse mammary carcinoma cells. In addition, it has been shown [336], that either spermidine or spermine derivatives can support growth of polyamine depleted SV-3T3 cells without the need for metabolic conversion. Furthermore, cytostasis of mammalian cells induced by the ornithine decarboxylase (ODC) inhibitor DFMO or a S-adenosylmethionine decarboxylase inhibitor [530] has been reported to have two phases, an acute initial phase due to reduction of total cellular polyamines and a delayed second phase due to eventual deprivation of elF5A. In fungi, polyamines are essential for distinct differentiation processes, such as spore germination, sporulation and dimorphism [395]. In Ustilago maydis [170] and Yarrowia lipolytica [221], polyamine concentrations higher than those necessary to ensure vegetative growth are required to sustain dimorphic transition. It has been reported that polyamines, mainly putrescine, might play an active role in the pathosystem maize $-U$. maydis [389].

Transcriptional regulation of the ODC-encoding gene in higher eukaryotes occurs under different conditions [68]. In fungi contrasting data exist. Evidence of transcriptional regulation of the homolog genes has been described in Neurospora crassa Spe1p [206] and Mucor circinelloides [35], whereas no transcriptional regulation of the corresponding genes was observed in $U$. maydis [170], Candida albicans [285] and Coccidioides immitis [169], during the dimorphic transition.

The possible significance of the transcriptional regulation by polyamines of the gene encoding $O D C$ in fungi may depend on its interrelation with the post- 
translational regulation of the enzyme. This offers a rapid response of the cell to the environmental conditions, but is energetically expensive, since it depends on the inactivation of $O D C$ [67]. Transcriptional regulation would respond more slowly, but would save energy to the cell, controlling the amounts of transcript and enzyme. The simultaneous operation of both mechanisms would provide a more efficient mechanism for the control of the cellular functions that depend on the levels of polyamines. Jiménez-Bremont and Ruiz-Herrera in 2008 by analysis of the transcriptional regulation of $Y L O D C$ gene from the dimorphic fungus yarrowia lipolytica reported that there exists a weak but reproducible transcriptional regulation of YIODC under the analyzed conditions. Their data suggest that gene transcription is down-regulated by putrescine, and stimulated by spermidine. It is suggested that the simultaneous operation of transcriptional and post-translational regulatory mechanisms endows the cell with a more efficient mechanism to control the physiological processes that depend on polyamines [220]. Accordingly, whether transcriptional regulation of the ODC-encoding genes is widespread, and how regulation occurs, remains unknown. In this work, we report a study of the transcriptional regulation of the gene encoding $O D C$ in the dimorphic fungus $C$. albicans (Chapter IV).

\section{Polyamine auxotrophs of Candida albicans}

Strains of $C$. albicans have been constructed that are unable to synthesize ornithine and are thereby deficient in polyamine biosynthesis. This strain has been used to develop a protocol for observation of mutant phenotype cells under varius condition that blocked directly in polyamine synthesis. It has been demonstrated previously in our laboratory that low concentrations of putrescine $(0.01 \mathrm{mM})$ are sufficient for near-normal growth of an odc mutant polyamine auxotroph of $C$. albicans. Homozygous null Candida mutant grew exclusively in the yeast form at low polyamine levels $(0.01 \mathrm{mM}$ putrescine) under all conditions tested. An increase in the polyamine concentration (10 $\mathrm{mM}$ putrescine) restored the capacity to switch from the yeast to the filamentous form. The strain with a deletion mutation also showed increased sensitivity to salts and Calcofluor white. This Candida odc/odc mutant was virulent in a mouse model. The results suggest a model in which polyamine levels exert a pleiotrophic effect on transcriptional activity, and that polyamine levels control a broad range of gene functions [188]. 



\section{Transcriptional response of Candida albicans to temperature}

\section{Abstract}

One of the main parameters involved in the yeast-to-hypha transition in Candida albicans is temperature and this transition is involved in pathogenicity. A complete switch between both forms -yeast and hypha- can be achieved by changing temperature from $28^{\circ} \mathrm{C}$ to $37^{\circ} \mathrm{C}$ in minimal medium; namely, Lee medium. Here we carried out a genome-wide analysis of the response of $C$. albicans to temperature after 15,60 and 180 min exposure to $37^{\circ} \mathrm{C}$. Using a cDNA microarray method, a total of 781 genes were found to change at the consensus level of $\geq 1.5$-fold, including 401 up-regulated genes and 380 down-regulated genes. 341, 403 and 251 genes changed after 15,60 , and 180 min of incubation at $37^{\circ} \mathrm{C}$, suggesting that the key time for controlling the temperature switch and the dimorphic change occurs very early and that mainly housekeeping hyphal genes are expressed after three hours.

Of those genes, 43 were common to the three times assayed (24 and 19 up- and down- respectively); 68 and 52 genes were common between 15 and $60 \mathrm{~min}$. and 60 and 180 min., respectively, and variations in 222, 242 and 148 genes were found only at one time of induction (15, 60 and $180 \mathrm{~min}$. respectively). Only 8 genes were common to 15 and $180 \mathrm{~min}$. indicating a sequential gene expression and confirming the validity of our approach. Of the genes common for the three times assayed, 3 corresponded to genes coding for proteins of unknown function. The main overrepresented categories were cell wall genes with 9 genes (seven up, ALS1, ALS3, ECE1, HWP1, PHR1, RBT1, SUN41 and two down-, PIR1 and YWP1), transporters CA3966, ENA21, ENA2, GAP6 and PHO84 up- and four down-, MEP2, PMA1 and OPT2 and OPT3 (two oligopeptide transporters; genes induced transcriptionally) upon phagocytosis by macrophages; and fluconazoleinduced, dehydrogenases (ARD, PUT2 and GDH3), and oxidase/peroxidaseencoding genes ( $A M O 1, A O X 2$ and GPX2). We also detected the down-regulation of three glycolytic genes (PDC11, PFK1 and PFK2) and the up- and downregulation of two transcription factors: GAT2 and STP3 (the latter of which regulates $S A P 2)$. The main categories of common genes after 15 and 60 min of incubation at $37^{\circ} \mathrm{C}$ belonged to the following categories: ribosomes (ERF1, RPL25, RPL33, RPS10 and RPS13) and glycolysis (ADH1, ADH2, ENO1, FBA1, PGI1, $P G K 1$ and TPI1). Between 60 and $180 \mathrm{~min}$, the main categories of common genes 
corresponded to thiamine biosynthesis (THI4, THI6 and THI13) and drug resistance proteins (CDR1, CDR2 and CDR4). Representative categories of genes regulated at only one time were: (15min.) ergosterol biosynthesis (ERG1, ERG3, ERG10, ERG11, ERG20 and ERG251), ferroxidases and ion transporters (FET3, FET99, FTH1, FTR1 and FTR2), and histones (HHF1, HHF22, HHT1, HHT21, HTA3 and HTB1); at 60 min: general amino acid permeases (GAP1, GAP4 and HIP1), lipases (LIP7, LIP8 and LIP9) and ribosomes (RPL18, RPL24A, RPL38, RPS4A, RPS20 and RPS22A). Under our conditions, only a few transcription factors were detected, indicating that either shorter times of incubation should be assayed or that the limit of fold-change imposed should be changed.

\section{Introduction}

Candida albicans is a polymorphic organism able to grow in several yeast forms either in liquid media [431] or switching spontaneously in solid media to form colonies with different phenotypes [430]. C. albicans is also able to form chlamydospores that may allow survival under harsh environmental conditions, acting as resting forms, as has been described for other species, although this assumption and their putative role in infection remain to be confirmed $[298,446]$. Finally, C. albicans can undergo a dramatic change in morphogenesis due to environmental conditions when round yeast cells form pseudohyphal and true filaments, and both processes, which are controlled by a complex network of parallel pathways, play a role in virulence [34,451]. The well established DNA array technology allows efficient analysis of the genome-wide expression of multiple strains, mutants, and different growth conditions. Much work has been published about issues such as the yeast-hypha transition [342], cyclic AMP signalling [177], the regulation of morphogenesis and metabolism by APSES proteins [108], the role of the FLO8 transcription factor in hyphal development [54], the convergent regulation of virulence by Cph1p, Cph2p and Efg1p [261], the Tac1 regulon [282], the response to steroids [21], etc. Despite such a large body of information, we still lack the transcriptomes of $C$. albicans obtained under standard and well defined sets of conditions, and neither has the kinetics of induction of the regulatory and structural genes yet been unravelled. In the present work, we analyzed the response of $C$. albicans in a minimal medium (Lee) dependent only on temperature at three induction times: 15, 60 and $180 \mathrm{~min}$. We detected a peak in gene alteration 
at 60 min., suggesting that most of the regulatory processes have been completed at this time and that at more prolonged times mainly housekeeping genes are expressed. In similar approaches, Nantel et al [342] found a regulated expression of 232 genes (up- and down-regulated) by comparison of the growth of $C$. albicans in several media, and Kadosh and Johnson [226] described 61 genes that were induced more than two-fold during the blastospore-to-filament transition. At all three incubation times assayed, we detected the regulation of most of the cell wall genes described by other authors [226,342] -ECE1, HWP1, PHR1 and RBT1- although two exceptions were remarkable. We observed a putative change in the transcriptional regulator of the SAP family, not in the genes of that family, and we detected the coordinated expression of the genes involved in pathogenicity: the ALS and LIP families [201,410]. At least at one time, we also found 15 genes (out of 46) described specifically by Nantel et al [342] and we observed the regulation of three new families: BMT, the $F_{1} F_{0}$ ATPase complex, and THI. Several important sets of genes appeared misregulated at one of the times assayed; i.e. genes coding for histones, for general amino acid permeases (after 15 and $180 \mathrm{~min}$. of incubation respectively), etc. Our results indicate that for modelling the yeast-hypha transition in C. albicans further experimental approaches with defined sets of parameters should be addressed.

\section{Materials and methods}

The strain of C. albicans used was CAl4, $\Delta$ ura3::imm434/4ura3::imm434 [132]. It was maintained by periodic transfer to slants of yeast extract-dextrose (YEPD) medium (1\% yeast extract, $1 \%$ glucose, $2 \%$ agar). Yeast growth was achieved in YNB (0.67\% yeast nitrogen base (Difco), 0.5\% ammonium sulphate, $0.2 \mathrm{mM}$ uridine, $1 \%$ glucose) or Lee medium [270] containing $1.25 \%$ glucose as a carbon source and supplemented with $0.2 \mathrm{mM}$ uridine, at $28^{\circ} \mathrm{C},(\mathrm{pH} 6.8)$. The dimorphic transition was induced by changing the temperature to $37^{\circ} \mathrm{C}$ in Lee medium. Cells were photographed using a Zeiss Axiophot phase-contrast photomicroscope equipped with a $35 \mathrm{~mm}$ camera using llford FP4 Plus film (125 ASA).

\section{RNA isolation, cDNA preparation and microarray hybridization}

C. albicans strain CAl4 was grown in MM or under our condition for the yeasthypha transition. Cells were pre-grown in Lee medium up to the exponential phase 
at $28^{\circ} \mathrm{C}$, washed, starved in water for $24 \mathrm{~h}$ at $4^{\circ} \mathrm{C}$, and then incubated in Lee medium at $37^{\circ} \mathrm{C}$. Samples $\left(300 \mathrm{ml}\right.$ of cells, $10^{7}$ cells $\left./ \mathrm{ml}\right)$ were collected at 15,60 and 180 min., harvested at room temperature, resuspended in a very small volume of the supernatant, and immediately frozen by releasing small drops of cell samples into liquid nitrogen. Cells were then stored at $-80^{\circ} \mathrm{C}$ until RNA extraction. Total RNA was isolated by breaking cells in a micro-dismembrator (Braun, Melsungen), followed by the trizol extraction method, as described in the Galar Fungail standard $\begin{array}{llll}\text { operating } & \text { procedures } & \text { for } & \text { extraction }\end{array}$ (http://www.pasteur.fr/recherche/unites/Galar Fungail/), keeping the cells frozen in liquid nitrogen at all times. RNA was checked for integrity by electrophoresis and quantified by spectrophotometric analysis at $260 \mathrm{~nm}$.

About $15 \mu \mathrm{g}$ of total RNA was resuspended in $20 \mu$ of RNase-free $\mathrm{H}_{2} \mathrm{O}$ along with $1 \mu \mathrm{l}$ of RNasin ribonuclease inhibitor (Promega) and then used for labelled cDNA synthesis, following a protocol from Eurogentec, with minor modifications. Direct labelling reactions were carried out by reverse transcription using the SuperScript II $\mathrm{RT}^{\mathrm{TM}}$ enzyme (Invitrogen), the Candida albicans specific primer mix (Eurogentec), OligodT (12-18) (Sigma), the dNTP mix (Sigma) and Cyanine 3-dCTP/Cyanine 5dCTP (Amersham Biosciences), DTT and 5× First-strand buffer. In short, total RNA isolated from C. albicans cells was used to obtain cDNA labelled with Cyanine 3dCTP or Cyanine 5-dCTP by a reverse transcription reaction lasting 3 hours. Then, the reaction was stopped by the addition of EDTA, and the residual RNA was denatured by adding $\mathrm{NaOH}$ and incubating at $65^{\circ} \mathrm{C}$ for $20 \mathrm{~min}$. The labelled probe was then purified using the Qia-quick PCR purification kit (Qiagen). cDNA was checked for proper labelling by measuring the amount of probe and incorporated dye and calculating the frequency of incorporation (f.o.i). Only probes with more than 65 pmol of dye incorporated and an f.o.i greater than 20 labelled nucleotides per 1000 nucleotides were used for subsequent microarray hybridization. From each probe, the volume corresponding to $65 \mathrm{pmol}$ of dye was concentrated to an approximate volume of $5 \mu \mathrm{l}$ using Microcon-30 filters (Amicon). Concentrated Cy5/Cy3-labelled probes from treated and untreated cells were mixed. Then, $5 \mu \mathrm{l}$ of denatured salmon sperm DNA $(10 \mathrm{mg} / \mathrm{ml})$ was added and after DNA denaturing DIG easy hybridization buffer (Roche) was incorporated to a final volume of $60 \mu$ l. The probe was hybridized onto the microarray underneath a coverslip (Sigma) and incubated overnight at $42^{\circ} \mathrm{C}$ in a Corning chamber. Following this, the microarrays 
were washed following the Eurogentec recommendations and dried by centrifugation at $1100 \mathrm{rpm}$ at room temperature.

\section{cDNA microarray data analysis}

Transcriptional profiling was carried using cDNA microarrays containing 6039 C. albicans ORFs of strain SC5314 ( 98\% of total) in duplicates purchased from Eurogentec (Seraing, Belgium). The data analysed included 4 replicates, corresponding to 2 biological replicates (RNA from independent cultures), and a dye swap experiment for each one. Since the ORFs were spotted in duplicate onto each microarray, each gene was represented by eight datasets. The microarrays were scanned with a GenePix 4000B scanner, and intensity data were captured with GenePix Pro, version 4.0. Each microarray image was inspected to identify low-quality spots that were not included in the analysis. Normalization and statistical analyses were performed with GeneSpring software, version 5.0.3 (Silicon Genetics/Agilent Technologies, Redwood City, CA). Data were per-chipand per-spot-normalized by intensity-dependent (Lowess) normalization applied to the print-tip region. Genes with differential expression were obtained by first filtering genes with a fold-change $\geq 1.5$ in at least one hybridisation and then applying a "statistical group comparison" (Wilcoxon-Mann-Whitney test) and the multiple testing correction Benjamini and Hochberg False Discovery Rate (FDR) with a pvalue $\leq 0.02$. The first filter was introduced to control the stringency of the FDR correction, which is directly influenced by the number of genes analysed. Among the genes selected by the test, we considered genes as being significantly regulated only if they were regulated by at least a mean factor of 1.5. Cluster analyses and Venn diagrams were carried out with the GeneSpring program, using standard value settings. Gene designations were in accordance with the annotation of the Candida DB Web server (http://genolist.pasteur.fr/CandidaDB/) and the Candida Genome Database (http://www.candidagenome.orgh). Total gene expression data are available in the Supplementary material.

\section{Results}

\section{Morphology of the cultures}

The yeast-hypha transition was induced from cells pre-grown in Lee medium up to the exponential phase at $28^{\circ} \mathrm{C}$, washed, starved in water for $24 \mathrm{~h}$ at $4^{\circ} \mathrm{C}$, and then 
incubated in Lee medium at $37^{\circ} \mathrm{C}$. Under these conditions, the growth of most of the cells was arrested in the $\mathrm{G} 1$ phase of the cell cycle, and a reproducible and homogeneous hyphal formation was obtained, Figure 2.1.
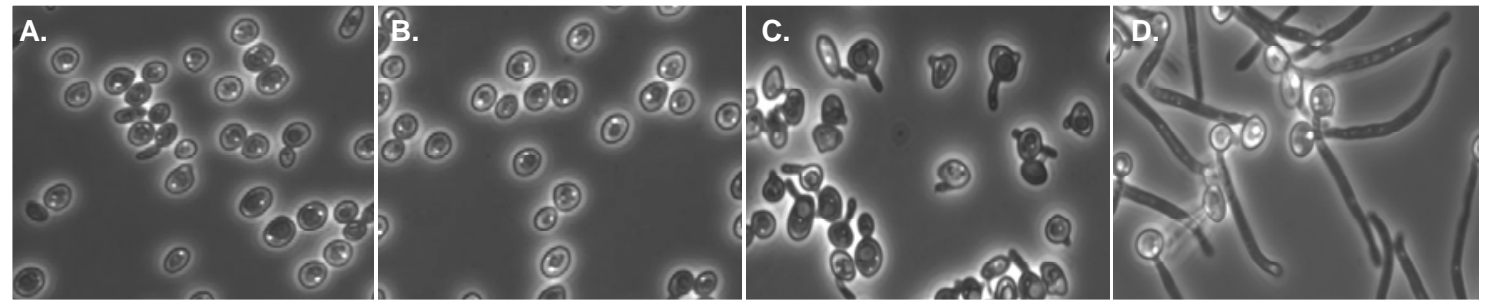

Figure 2.1 Morphology of Candida albicans. Yeast phenotype of the cells of the inoculum incubated in Lee medium up to the exponential phase at $28^{\circ} \mathrm{C}$, washed and starved in water for $24 \mathrm{~h}$ at $4^{\circ} \mathrm{C}(\mathrm{A})$. Yeast-hypha transition after 15 (B), 60 (C) and $180 \mathrm{~min}(\mathrm{D})$

\section{Global gene expression profile}

In previous studies, the transcriptomes of the C. albicans SC5314 and CAF2-1 strains were compared by induction of the yeast-hypha transition in YPD at $30^{\circ} \mathrm{C}$, YPD plus serum at $37^{\circ} \mathrm{C}$ after $30 \mathrm{~min}$, at 1 and 6 hours of induction, and at 25$37^{\circ} \mathrm{C}$ and at $30-37^{\circ} \mathrm{C}$ temperature shifts in the absence of serum [342], or by growing in YEPD at 1, 2, 3 and 5 hours [226]. As a means to identify the genes responding only to temperature, and in order to analyze the behavior of these, we performed gene expression profiling analysis at three shorter times of incubation at $37^{\circ} \mathrm{C}-15,60$ and 180 min.- by comparison with yeast growing in the exponential phase at $28^{\circ} \mathrm{C}$. A total of 781 genes were found to change at the consensus level of $\geq 1.5$ fold, including 401 up-regulated genes, and 380 down-regulated genes. The distribution of genes altered at the three time-points is represented in Figure 2.2, and the Venn diagram and the clustering of genes regulated by temperature are shown in Figure 2.3 ( $A$ and $B$ ).

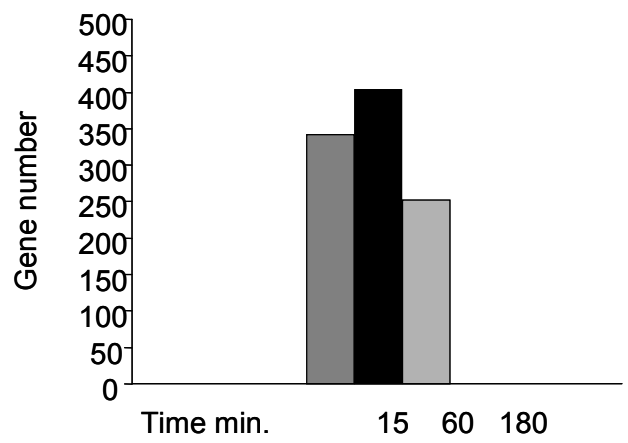

Figure 2.2 Total number of genes regulated by temperature at the $\geq 1.5$-fold level 
A

Up-regulated genes

132 genes (15 min.) 250 genes (60 min.)

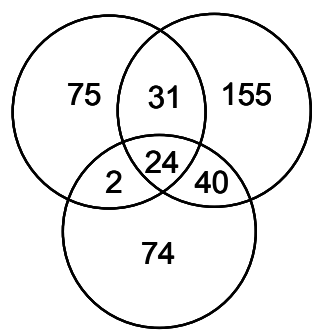

140 genes (180 $\mathrm{min}$.)

Down-regulated genes

209 genes (15 min.) 153 genes (60 min.)

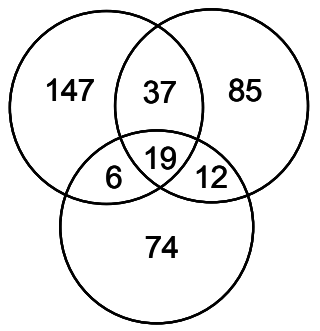

111 genes (180 $\mathrm{min}$.)
B

CAI 4

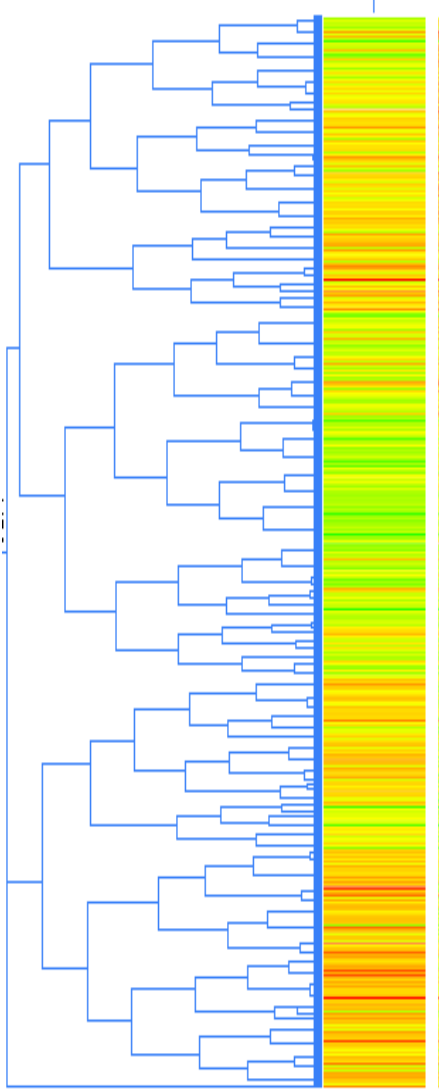

Time min

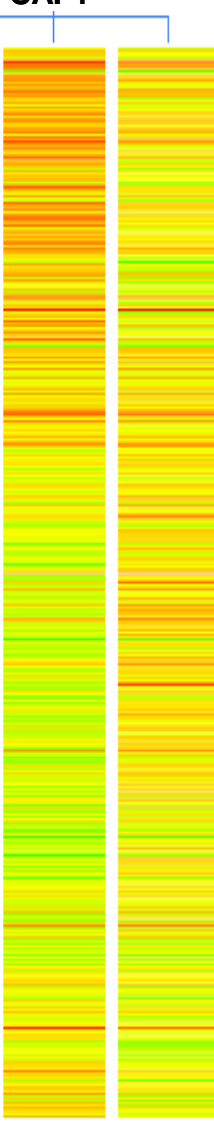

$15^{\prime}$

$60^{\circ} 180^{\circ}$

Figure 2.3 Genes regulated by temperature. (A) Venn diagram of up-regulated and down-regulated genes, including those common to all three times studied. (B) Clustering of genes regulated by temperature. Ratios of gene expression obtained by dividing the experimental samples by the reference samples are represented with a red-green colour scale.

The time-course of gene expression clearly indicates a peak of regulated genes after 60 min., suggesting that most of the signals and the regulatory genes involved in the morphogenetic switch exert their function rapidly. We observed a good statistical correlation in our results; thus, 43 genes were common to the three times assayed (24 and 19 up- and down- respectively), 68 genes at 15 and 60 min (31 up- and 37 down-) and 52 genes at 60 and 180 min. (40 up- and 12 down-). 222 genes (75 up- and 147 down-), 240 genes (155 up- and 85 down-), and 148 genes (74 up- and 74 down-) varied only at one induction time (15, 60 and $180 \mathrm{~min}$. respectively). However, only 8 genes were common between 15 and $180 \mathrm{~min}$. validating our experimental approach. The elevated number of regulated genes detected only at one time suggests a coordinate expression of genes and gene families and a rapid adaptation to temperature and to the morphogenetic switch. The 43 genes common to the three times are listed in Table 2.1. 
Table 2.1 Genes whose transcription is induced more than 1.5 fold at $37^{\circ} \mathrm{C}$ at the three times assayed, 15,60 and $180 \mathrm{~min}$.

\begin{tabular}{|c|c|c|c|c|c|}
\hline $\begin{array}{l}\text { Gene } \\
\text { name }\end{array}$ & \multirow[t]{2}{*}{ Accession no } & \multirow[t]{2}{*}{ Description (CandidaDB) } & \multicolumn{3}{|c|}{ Fold regulation } \\
\hline UP-regulated genes & & & $15 \mathrm{~min}$ & $60 \mathrm{~min}$ & $180 \mathrm{~min}$ \\
\hline \multicolumn{6}{|c|}{ Cell wall proteins } \\
\hline ALS1 & orf19.5741 & Agglutinin-like protein & 20.99 & 5.17 & 3.00 \\
\hline ALS3 & orf19.1816 & Agglutinin like protein & 2.23 & 11.44 & 3.5 \\
\hline ECE1 & orf19.3374 & Cell Elongation Protein & 22.72 & 26.57 & 14.00 \\
\hline HWP1 & orf19.1321 & Hyphal wall protein & 6.43 & 16.97 & 11.60 \\
\hline PHR1 & orf19.3829 & GPI-anchored $\mathrm{pH}$ responsive glycosyl transferase & 2.98 & 3.46 & 1.90 \\
\hline RBT1 & orf19.1327 & Putative cell wall protein with similarity to Hwp $1 \mathrm{p}$ & 1.62 & 4.10 & 2.85 \\
\hline SUN41 & orf19.3642 & Putative cell wall beta-glucosidase (by homology) & 1.67 & 2.66 & 1.95 \\
\hline \multicolumn{6}{|c|}{ Dehydrogenases } \\
\hline ARD & orf19.6322 & D-arabinitol dehydrogenase & 1.85 & 1.83 & 2.17 \\
\hline PUT2 & orf19.3974 & 1-pyrroline-5-carboxylate dehydrogenase & 2.24 & 1.84 & 2.22 \\
\hline \multicolumn{6}{|c|}{ Oxidases/peroxidises } \\
\hline $\mathrm{AOX} 2$ & orf19.4773 & Alternative oxidase (by homology) & 1.54 & 1.72 & 1.74 \\
\hline GPX2 & orf19.85 & Glutathione peroxidase (by homology) & 3.60 & 3.63 & 3.80 \\
\hline \multicolumn{6}{|c|}{ Transporters } \\
\hline ENA21 & orf19.5170 & P-type ATPase & 2.93 & 2.15 & 1.96 \\
\hline ENA2 & orf19.6070 & P-type ATPase involved in $\mathrm{Na}^{+}$efflux & 2.12 & 2.10 & 1.70 \\
\hline GAP6 & orf19.6659 & General amino acid permease & 1.63 & 2.40 & 2.19 \\
\hline IPF9376 & orf19.655 & High-affinity inorganic phosphate $/ \mathrm{H}^{+}$symporter & 2.49 & 3.95 & 2.09 \\
\hline \multicolumn{6}{|c|}{ Transcription } \\
\hline GAT2 & orf19.4056 & Putative DNA-binding transcription factor & 1.73 & 2.39 & 1.82 \\
\hline \multicolumn{6}{|c|}{ Other functions } \\
\hline ARP3 & orf19.2289 & Actin related protein (by homology) & 2.25 & 2.90 & 2.18 \\
\hline DCK1 & orf19.8435 & Putative guanine nucleotide exchange factor & 3.81 & 2.75 & 1.81 \\
\hline DEF1 & orf19.7561 & Protein required for filamentous growth & 1.76 & 2.11 & 1.80 \\
\hline IPF1172 & 5 orf19.10979 & A Gag-related protein; hyphal induced & 2.82 & 2.94 & 3.01 \\
\hline PCK1 & orf19.7514 & Phosphoenolpyruvate carboxykinase & 1.99 & 5.76 & 3.64 \\
\hline TMA19 & orf19.3268 & Ortholog of S. cerevisiae Tma19p (Ykl065cp) & 1.74 & 1.92 & 1.73 \\
\hline \multicolumn{6}{|c|}{ Unknown function } \\
\hline PET18 & orf19.7330 & Unknown & 3.95 & 7.43 & 1.64 \\
\hline \multicolumn{6}{|c|}{ Down-regulated genes } \\
\hline \multicolumn{6}{|c|}{ Cell wall proteins } \\
\hline PIR1 & orf19.220 & Putative cell wall protein of the PIR family & 0.30 & 0.24 & 0.35 \\
\hline YWP1 & orf19.3618 & Putative cell wall protein (by homology) & 0.28 & 0.25 & 0.16 \\
\hline \multicolumn{6}{|c|}{ Dehydrogenases } \\
\hline GDH3 & orf19.4716 & NADP-glutamate dehydrogenase & 0.15 & 0.18 & 0.40 \\
\hline \multicolumn{6}{|c|}{ Oxidases/peroxidases } \\
\hline AMO1 & orf19.5784 & Amine oxidase (by homology) & 0.44 & 0.30 & 0.38 \\
\hline \multicolumn{6}{|c|}{ Transporters } \\
\hline MEP2 & orf19.5672 & Ammonium permease & 0.26 & 0.18 & 0.35 \\
\hline OPT2 & orf19.3746 & Oligopeptide transporter & 0.34 & 0.40 & 0.23 \\
\hline OPT3 & orf19.3749 & Oligopeptide transporter & 0.45 & 0.51 & 0.51 \\
\hline PMA1 & orf19.5383 & Plasma membrane $\mathrm{H}^{+}$-transporting ATPase 1 & 0.40 & 0.50 & 0.56 \\
\hline \multicolumn{6}{|c|}{ Glycolysis } \\
\hline PDC11 & orf19.2877 & Pyruvate decarboxylase (by homology) & 0.33 & 0.19 & 0.60 \\
\hline PFK1 & orf19.3967 & 6-phosphofructokinase alpha subunit & 0.25 & 0.56 & 0.51 \\
\hline PFK2 & orf19.6540 & 6-phosphofructokinase beta subunit & 0.23 & 0.58 & 0.48 \\
\hline \multicolumn{6}{|c|}{ Transcription } \\
\hline STP3 & orf19.5917 & Transcription factor & 0.59 & 0.52 & 0.53 \\
\hline Other fu & nctions & & & & \\
\hline DUR1.2 & orf19.780 & Urea amidolyase (by homology) & 0.60 & 0.46 & 0.52 \\
\hline FET99 & orf19.11689 & Cell surface ferroxidase (by homology) & 0.23 & 0.33 & 0.46 \\
\hline
\end{tabular}




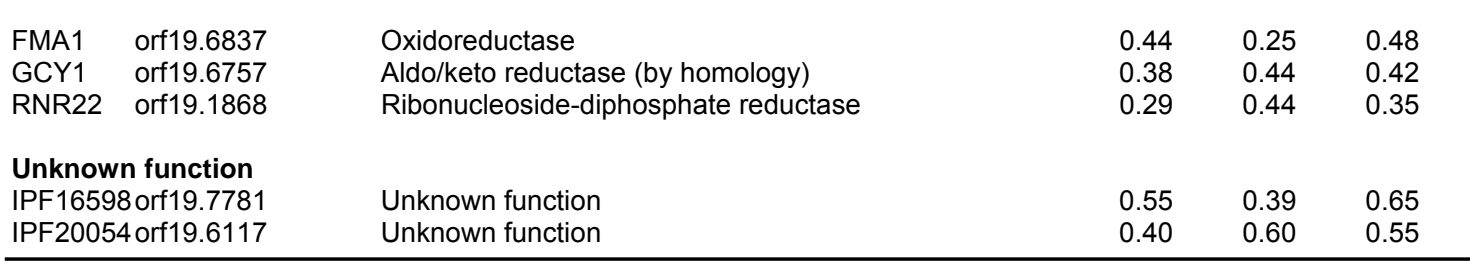

The lowest fold-change detected was 0.15 for $G D H 3$ at $15 \mathrm{~min}$. and the highest fold-change was 26.57 for ECE1 at $60 \mathrm{~min}$. These fold-changes match perfectly in the range of our assays (see Supplementary material Tables 2.1 and 2.2). Only 3 of the common genes corresponded to genes of unknown function. This percentage, roughly $7 \%$, is one third of those found at global level (25\%), as would be expected from our knowledge about the morphogenetic switch. Of the rest of the genes, 10 belonged to disperse functional categories; i.e., $A R P 3$, actin-related protein; $R N R 22$, ribonucleoside diphosphate reductase, etc., and 27 genes could be grouped in six functional categories. The main one corresponded to cell wall proteins and included the up-regulation of HWP1, a well known cell wall protein [443]; ECE1, a protein involved in cell elongation [27,32]; PHR1, a cell surface glycosidase [131], and RBT1 [27,434], a putative cell wall protein with similarity to Hwp1p. We observed the down-regulation of YWP1, a cell wall and secreted protein [159], and of PIR1, a cell wall structural protein [309]. All these proteins have been described previously as being up- and down-regulated at similar levels of induction [226,342] and are regulated by Efg1p and Tup1p [108,226,434].

Also well represented were the following categories: transporters with 9 genes, including the two oligopeptide transporters, oxidases/peroxidses (4 genes), glycolysis (3 genes), and dehydrogenases (3 genes) (see below). Our results indicate an important bias toward the functional categories mentioned previously and show that the role of these genes in the yeast-hypha transition must be investigated more carefully.

\section{Expression of transcription factors}

Only one transcription factor was up-regulated at the three times of incubation; namely, GAT2, which has been described as a putative DNA-binding transcription factor with an unspecified role in morphogenesis that awaits further characterization. Three transcription factors -CLN21, HAC1 and UME6- were upregulated at two times: 15 and $60 \mathrm{~min}$. CLN21 is a hypha-specific G1 cyclin protein involved in the regulation of hyphal morphogenesis [428,543]; HAC1 is a putative 
transcription factor involved in unfolded protein response and the control of cell morphology [508], and UME6 is a transcription factor required for wild-type hyphal extension [22,282]. Deletion of all three genes produces clear defects in hyphal formation and extension, and hence our results are in agreement with previous ones. However CLN21 is also induced after overexpression of EFH1 [108] and HAC1 is down-regulated in an efg1, efh 1 mutant [108], suggesting that the regulatory circuits described to date are incomplete [34] and that our knowledge of them must be improved. The other up-regulated transcription factors (at $60 \mathrm{~min}$. of incubation) were SKO1, a putative transcription factor involved in the cell wall damage response [379]; TEC1, a TEA/ATTS transcription factor involved in the regulation of hypha-specific genes [262], and UME7, a putative transcription factor with a zinc cluster DNA-binding motif [298]. At $180 \mathrm{~min}$. of incubation we found ZCF20, a predicted zinc-finger protein of unknown function [298], to be regulated UME7 and ZCF20 have been characterized only by "in silico" analysis [298]. The expression of TCC1, a protein involved in the regulation of filamentous growth and virulence that interacts with Tup $1 p$ and acts in the transcriptional regulation of hypha-specific genes [229], and CTA2, a putative transcriptional activator downregulated by Efg1p [108], was increased from 0.65 to 1.29 and from 0.50 to 5.50 (at $15 \mathrm{~min}$. to $180 \mathrm{~min}$. of incubation respectively). Both results again perfectly match the hypothesis of a coordinated developmental program. Of the 6 down-regulated transcription factors, only one appeared regulated at all three times; namely, STP3, which regulates SAP2 [311] (see below). STP4, a putative transcription factor with a zinc-finger DNA-binding motif, was regulated at $60 \mathrm{~min}$. [46]. At $180 \mathrm{~min}$. of incubation at $37^{\circ} \mathrm{C}$ we found: $H A P 2$, a CCAAT-binding factor that regulates low-iron (chelation) induction of FRP1 transcription [15]; orf19.173, with a zinc-finger DNAbinding motif [377] activated by MNL1 (which regulates weak acid-induced stress responses); MDM34, with a zinc-finger DNA-binding motif [129]; MNS4, similar to S. cerevisiae Msn4p [354] (all three putative transcription factors), and NRG1, a well known transcriptional repressor that regulates hyphal genes $[38,229,331]$.

\section{Time course of gene induction in the main categories detected}

In general, our results are in good agreement with those described by several authors [226,334,342,427], with only one remarkable exception; we were unable to detect the induction of the genes of the SAP family. This is probably due to the 
conditions we used to induce the morphogenetic switch. We only performed a change in temperature, and we were working with a minimal medium (Lee), while other authors changed temperature and either added serum or grew the culture in YPD medium. Our results are supported by the fact that the only transcription factor that was regulated at all three times was STP3, which in turn regulates SAP2 [311]: the enzyme of the family predominantly expressed under proteinase-inducing conditions in vitro $[211,339]$ and also the most important Sap enzyme in virulence [103]. To analyze the other regulated gene categories, we clustered together the main representative groups whose expression level changed at least at one time of incubation, by functional categories and all of them exhibited "kinetic" behavior.

Cell wall genes. Figure 2.4A shows the clustering of the cell wall genes affected. The ALS family includes 8 genes and of these, six changed their expression. Four increased their expression: ALS1 and ALS3 at all three times; ALS9 at two times (15 and $60 \mathrm{~min}$.), and ALS5 only at $60 \mathrm{~min}$. Our results revealed higher levels of expression for the $A L S 1$ and $A L S 3$ genes (up to 20 and 11 times respectively) and lower ones for the ALS9 and ALS5 genes (up to 2 and 1.5 times respectively), and they are in complete agreement with those described by other authors, who demonstrated the role of ALS3 in adhesion [541] and analysed ALS gene expression in budding and filamentous cultures of C. albicans by RT-PCR [164]. However, two of the ALS genes strongly decreased their expression; namely, ALS2 and ALS4 (Figure 2.4A).

Glucanases, glucosidases and mannosyltransferases. SUN41, a putative cell wall glucanase (the orthologue in S. cerevisiae is SUN4, [487]), was up-regulated at the three times of induction. Deletion of both alleles of the gene results in defects in hyphal formation [130] and a down-regulation of the gene during protoplast regeneration [62]. BGL2, which catalyzes the splitting (removing a disaccharide) and linkage of $\beta$-1,3-glucan molecules, was up-regulated at 60 and $180 \mathrm{~min}$. of incubation (microarray analysis revealed that BGL2 was induced during cell wall regeneration, its maximum value being reached after 2 hours [62]). BGL22 also showed increased induction kinetics, a value of 1.55 times being reached at 180 min. of incubation at $37^{\circ} \mathrm{C}$. A third putative glucanase, UTR2 [6], again regulated during protoplast regeneration [62], was also up-regulated. 


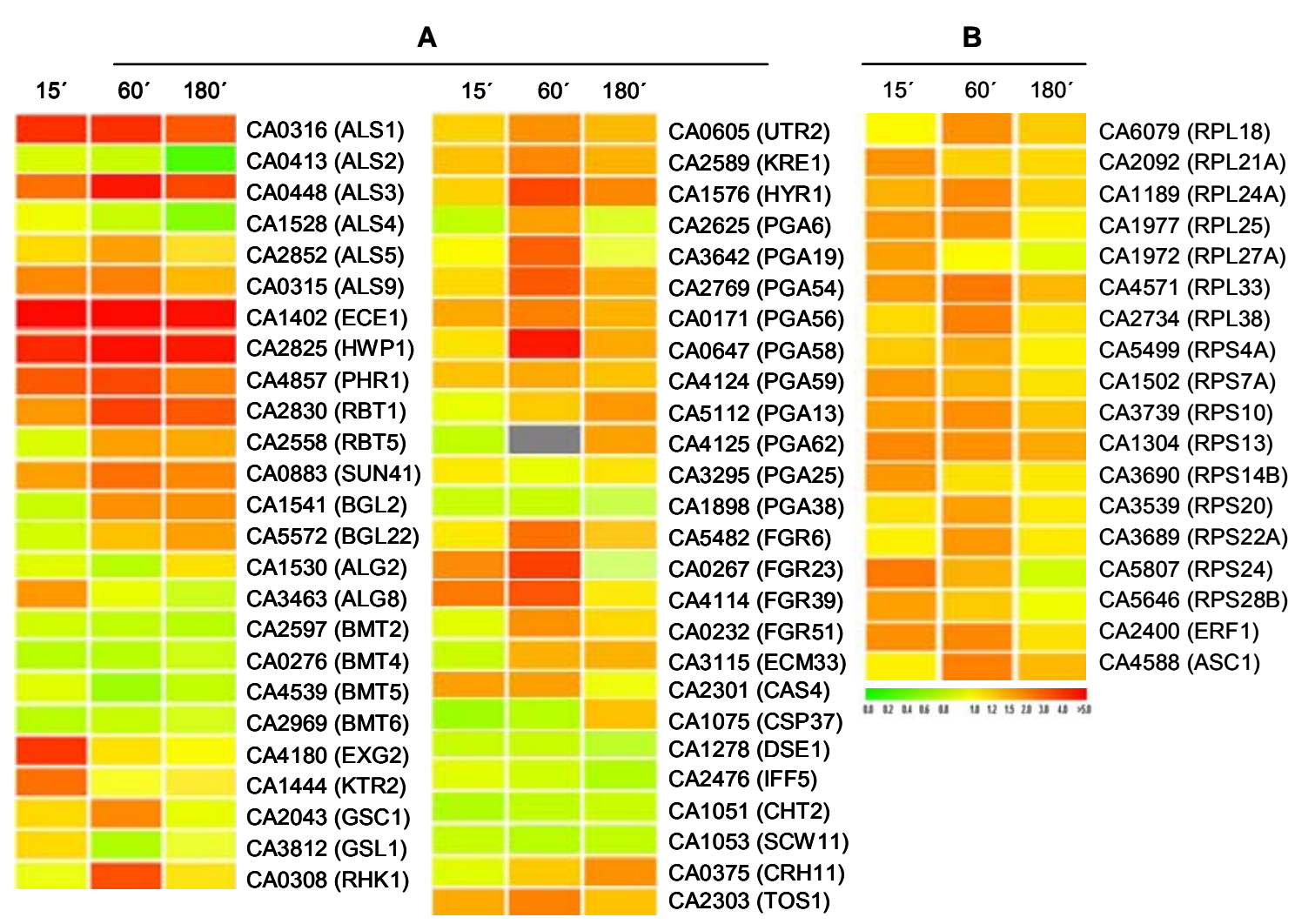

Figure 2.4 Transcriptional regulation by temperature. Cell wall, (A); and ribosomal, (B), genes Representative genes were grouped by gene families

More cell wall genes changed their expression: ALG2 and ALG8 (two putative glucosyltransferases involved in cell wall mannan biosynthesis), and GSL1 and GSL2, subunits of beta-1,3-glucan synthases [322], which showed a compensatory effect (see Figure 2.4). EXG2, an exo-1,3-beta-glucosidase induced during cell wall regeneration [62]; KTR2, a fungal-specific mannosyltransferase; $R H K 1$, a putative hitherto uncharacterized alpha-1,3-mannosyltransferase; CAS4, a protein the of RAM cell wall integrity signalling network required for hyphal growth [439], TOS1, a protein described as being similar to the alpha agglutinin anchor subunit [73], and DSC1 and SCW11, which are predicted cell wall proteins whose transcription is decreased in mutants lacking ACE2 [232]. Of interest was the down-regulation of several putative beta-mannosyltransferases: BMT2, BMT4, BMT5 and BMT6 [321]. Finally, CHT2, a chitinase GPI anchor induced in yeast-form cells [115,177], was down-regulated at all three times of incubation.

\section{GPI-anchored proteins}

A large group of genes coding for putative GPI-anchored proteins showed a single peak of induction at $60 \mathrm{~min}$ : KRE1 (also found to be highly regulated during $C$. 
albicans protoplast regeneration [62]); HYR1 (induced in hypha at 15 and $60 \mathrm{~min}$ ). [229], and RBT5, PGA6, 19, 54, 56, 58 and 59 [104] (PGA13 and PGA62 were also up-regulated, but at $180 \mathrm{~min}$.). We also observed the regulation of a set of genes coding for proteins lacking an orthologue in S. cerevisiae -transposon mutation affects filamentous growth- FGR 6, 23, 39 and 51 [477] (see Figure 2.4A); ECM33 (also modulated by adenylyl cyclase, [177]), and $C R H 11$, a predicted glycosyl hydrolase [104]. Very few cell wall genes were down-regulated: CSP37 (at 15 and 60 min.), PGA25 (at 60 min.), and PGA38 and IFF5 (at $180 \mathrm{~min}$.).

\section{Genes involved in protein synthesis and ribosomal proteins}

A second important category included the up-regulation of 18 genes coding for ribosomal proteins and involved in protein synthesis. Five of those genes were upregulated at 15 and $60 \mathrm{~min}$.; 6 genes at $15 \mathrm{~min}$., and the other 7 genes at $60 \mathrm{~min}$. (Figure 2.4B). No genes of this category were found up-regulated at $180 \mathrm{~min}$. or down-regulated at any time. Almost no data about the behavior of this group of genes under standard conditions of the yeast-hypha transition (serum or temperature) have been reported, and only RPS24 has been reported to be downregulated by cyclic AMP signalling [177,208,229,342]. Many of them have been described as up-regulated by comparison of different mutants with a wild-type strain: the Cacwt1 mutant [324] and the Caefg1 and Caefh1 mutants [108]. However, most of them are down-regulated upon $C$. albicans macrophage internalization [286].

\section{Central carbon metabolism}

The regulation of 40 genes (35 down-regulated after 15 min of incubation) involved in the glycolytic pathway, the TCA and glyoxylate cycles and gluconeogenesis (see Figure 2.5A, B and C) was detected. 


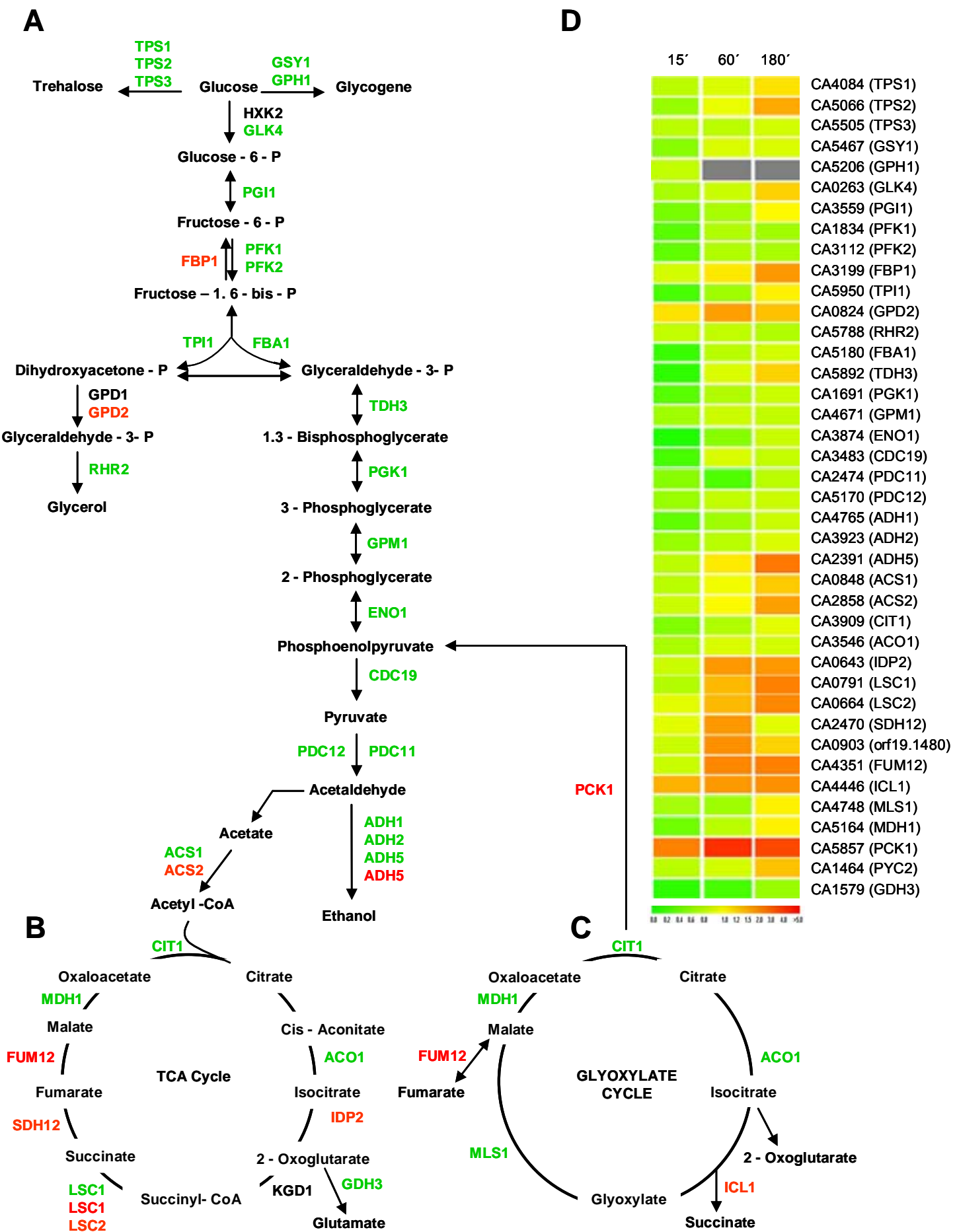

Figure 2.5 Transcriptional regulation by temperature of genes involved in glycolysis and gluconeogenesis $(\mathbf{A})$ and TCA (B) and glyoxylate (C) cycles. Green and red colours represent down- and up-regulation respectively.

The expression of all of them increased with time (Figure 2.5A), suggesting an adaptation to the hyphal mode of growth. In general our results are in agreement with those described by many authors [92,108,177,208,226,342,427], and they raise the question of the putative effect of mutations on the pathways [108]. Two 
genes involved in gluconeogenesis were up-regulated GPD2 and PCK1.

\section{Transporters and oligopeptide transporters}

Nine genes were regulated at the three times of incubation (four genes down- and five up-, Table 2.1) and 25 more at one or two times (Table 2.2).

Table 2.2 Regulated genes involved in transport

\begin{tabular}{|c|c|c|c|c|c|}
\hline \multirow[t]{2}{*}{ Gene name } & \multirow[t]{2}{*}{ Accession no } & \multirow[t]{2}{*}{ Description (CandidaDB) } & \multicolumn{3}{|c|}{ Fold regulation } \\
\hline & & & $15 \mathrm{~min}$ & $60 \mathrm{~min}$ & $180 \mathrm{~min}$ \\
\hline ALP1 & orf19.2337 & Amino-acid permease & 1.09 & 1.73 & 0.99 \\
\hline CTR1 & orf19.3646 & Copper transport protein & 1.07 & 1.09 & 1.76 \\
\hline DAL4 & orf19.313 & Probable uracile or allantoin permease & 0.84 & 0.66 & 0.82 \\
\hline DUR3 & orf19.781 & Putative urea transporter & 0.70 & 0.55 & 0.71 \\
\hline FEN2 & orf19.12981 & Predicted membrane transporter & 1.11 & 0.69 & 0.42 \\
\hline FET34 & orf19.4215 & Iron transport multicopper oxidase & 1.10 & 2.16 & 1.15 \\
\hline FTH1 & orf19.4802 & Iron transporter & 0.59 & 0.99 & 1.06 \\
\hline FTR2 & orf19.7231 & High affinity iron permease & 0.59 & 0.80 & 0.90 \\
\hline GAP1 & orf19.4304 & General amino acid permease & 1.14 & 2.90 & 1.24 \\
\hline GAP4 & orf19.4456 & General amino acid permease & 1.28 & 1.72 & 1.40 \\
\hline GNP1 & orf19.8784 & Asparagine and glutamine permease & 0.95 & 1.92 & 1.19 \\
\hline HAK1 & orf19.6249 & Putative potassium transporter & 0.72 & 0.71 & 0.62 \\
\hline HGT1 & orf19.4527 & High-affinity glucose transporter & 1.01 & 1.37 & 2.60 \\
\hline HGT7 & orf19.2023 & Sugar transporter & 0.57 & 0.74 & 0.94 \\
\hline HGT8 & orf19.2021 & Sugar transporter & 0.47 & 0.83 & 1.03 \\
\hline HIP1 & orf19.3195 & General amino-acid permease & 1.42 & 1.94 & 1.15 \\
\hline IPF14040 & orf19.2397 & Probable transporter & 1.90 & 1.41 & 0.94 \\
\hline IPF1992 & orf19.7336 & Predicted membrane transporter & 1.55 & 0.86 & 0.74 \\
\hline MAL31 & orf19.3981 & Maltose permease & 1.14 & 1.87 & 1.22 \\
\hline PHM7 & orf19.2170 & Membrane transporter & 0.81 & 2.13 & 1.33 \\
\hline PHO88 & orf19.7327 & Involved in phosphate transport & 1.52 & 1.31 & 1.20 \\
\hline PTR2 & orf19.6937 & Oligopeptide transporter & 0.60 & 1.23 & 1.07 \\
\hline SMF12 & orf19.2270 & Manganese transporter & 0.93 & 1.88 & 1.36 \\
\hline ZRT1 & orf19.3112 & High-affinity zinc transport protein & 1.04 & 0.96 & 2.08 \\
\hline ZRT2 & orf19.1585 & Zinc transport protein & 0.30 & 0.72 & 2.15 \\
\hline
\end{tabular}

A significant group of genes down-regulated at $15 \mathrm{~min}$. corresponded to iron transporters (FTH1, FTR1 and FTR2) [259]. Surprisingly, a regulation in the opposite way has been described for the two FTR genes [376]. The expression of FTR1 is induced under iron-limited conditions and repressed when the iron supply is sufficient, the opposite being observed for FTR2. FTR1 has also been reported as a virulence factor by the same authors [376] (Figure 2.6A). 


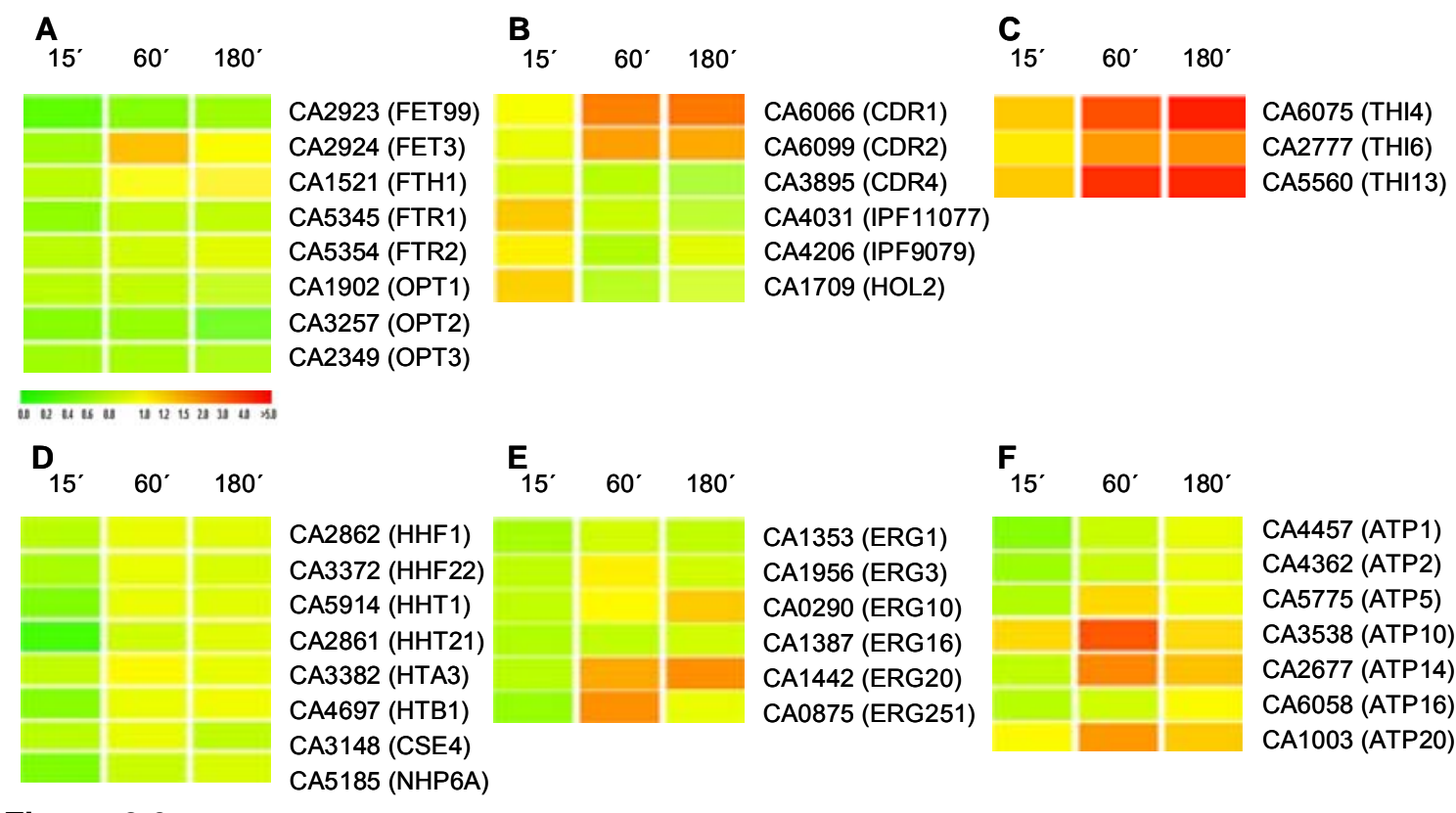

Figure 2.6 Transcriptional regulation by temperature of genes involved in transporters, (A); drug resistance, (B); thiamine biosynthesis, (C); histones, (D); ergosterol biosynthesis, (E); and $F_{1} F_{0}$ ATPase complex (F). Representative genes were grouped by gene families.

The genes up-regulated at $60 \mathrm{~min}$. include several amino acid permeases ( $A L P 1$, GAP1, GAP4, GNP1 and HIP1). Two oligopeptide transporters -OPT2 and OPT3were down-regulated at the three times of incubation. Another one, OPT1, was also down-regulated at 15 and $60 \mathrm{~min}$. of incubation [383] (Figure 2.6A). Another oligopeptide transporter, PTR2, with a discrepancy in the annotation -described as PTR21 (CA4707) or PTR2 (CA2690, CA2691)- was also detected.

\section{Drug resistance}

Three of the five putative genes belonging to the multidrug transporter of ATPbinding cassette $(A B C)$ superfamily were regulated at 60 and $180 \mathrm{~min}$. of incubation. Two of them -CDR1 and CDR2 [105]- were up-regulated and the other one, CDR4, with a less defined function, was down-regulated. Cdr1p and Cdr2p have an established role in conferring azole resistance [372] and both proteins also act as phospholipid translocases, which transport phospholipids in an in-to-out direction [432]. According to our data, it is tempting to speculate that the upregulation of drug transporters may favour the invasion of tissues by adapting cell membranes to a different composition. We also observed the down-regulation of CA1709, CA4031 and CA4206, three predicted membrane transporters, and members of the drug: proton antiporter (12 spanner, DHA1 family) of the major facilitator superfamily (MFS) [143] (Figure 2.6B). 


\section{Oxidases/peroxidases}

Two genes, AOX2 and GPX2, were up-regulated and one gene, AMO1, was downregulated at the three times of incubation. Other regulated genes were $A O X 1$, $P R X 1$ and two putative d-amino acid oxidases: IFG3 and IFG4. We detected two multicopper oxidases, FET3 and FET99, also required for growth under low-iron conditions [424].

\section{Hydrolases/Dehydrogenases}

We mainly detected the up-regulation of IMH3 (Inosine monophosphate (IMP) dehydrogenase [24]), SUR2 (a protein described as a ceramide hydroxylase); ALD5 (a protein described as an aldehyde dehydrogenase [177]); IFD6 and CSH1 (members of the aldo-keto reductase family, similar to aryl alcohol dehydrogenases [255]), and GDH2 (a putative NAD-specific glutamate dehydrogenase, fungalspecific; (no human or murine homolog), and the down-regulation of GND1, a putative 6-phosphogluconate dehydrogenase.

\section{Thiamine biosynthesis}

After 60 and $180 \mathrm{~min}$. of incubation, we detected the up-regulation of three genes involved in thiamine biosynthesis - THI4, THI6, and THI13- two of them (THI4 and THI6) being fungal-specific (Figure 2.6C). THI4, identified in S. cerevisiae as the gene involved in the synthesis of HET-P, is a single gene but its transcript is one of the most abundant under thiamine-depleted conditions [328]. Also, THI6 is a singlecopy gene and thi6 null strains have an absolute requirement for thiamine [357].

\section{Sets of genes regulated only at one incubation time}

After 15 and 60 minutes of incubation at $37^{\circ} \mathrm{C}$, several sets of genes were either up- or down-regulated. The main groups of up-regulated genes (at $15 \mathrm{~min}$.) included two RNA helicases; one of the DEAD box family; DBP2, and one ATPdependent RNA-helicase; namely, DBP3. The genes up-regulated at $60 \mathrm{~min}$ included lipases -LIP7, LIP8 and LIP9- while LIP10 was found to be downregulated. Among the down-regulated genes after $15 \mathrm{~min}$. of incubation, the following should be noted: histones (Figure 2.6D), with six genes (of the eleven genes coding for histones described in C. albicans: HHF1, HHF22, HHT1, HHT21, HTA3 and HTB1), including $\mathrm{H} 2 \mathrm{~A}, \mathrm{H} 2 \mathrm{~B}, \mathrm{H} 3$ and $\mathrm{H} 4$. We failed to detect the 
regulation of genes coding for histone $\mathrm{H} 1$. We also detected the down-regulation of CSE4, a centromere-associated protein; it is essential and is similar to CENP-A (centromeric histone $\mathrm{H} 3$ variant [383], and of NHP6A, a protein described as nonhistone chromatin component [281]. A second important category corresponded to genes involved in ergosterol biosynthesis (Figure 2.6E), with another six genes (ERG1, ERG3, ERG10, ERG11, ERG20 and ERG251). Both results suggest a rapid remodelling of chromatin, probably for it to be adapted to a new transcriptional profile, together with a change in membrane composition during hypha formation. The genes of the $\mathrm{F}_{1} \mathrm{~F}_{0}$ ATPase complex ATP1, ATP2, ATP5 and ATP16 were down-regulated at $15 \mathrm{~min}$. of incubation at $37^{\circ} \mathrm{C}$, a normal value being attained after $180 \mathrm{~min}$. of incubation. However, another set of genes of the same complex -ATP10, ATP14 and ATP20- displayed an induction peak at $60 \mathrm{~min}$. of incubation, [467] (Figure 2.6F). No specific sets of genes were found at $180 \mathrm{~min}$ of incubation, suggesting that at this time $C$. albicans has reached a general balance in its main regulatory metabolic pathways.

\section{Discussion}

In Candida albicans, a total of 781 genes out of the 6039 present in the microarray changed due to a variation of temperature $-28^{\circ} \mathrm{C}$ to $37^{\circ} \mathrm{C}$, in a minimal medium (Lee)- at one of the three incubation times assayed $(15,60$ and $180 \mathrm{~min}$.) at the arbitrary, although normally used, limit of $\geq 1.5$ fold. These 781 genes represent a percentage of $13 \%$. 198 genes, representing $25 \%$ of the genes that varied, were classified as being of unknown function, indicating that we need to improve our knowledge of the Candida genome, even as regards the morphogenetic process, which is one of the aspects most widely studied in this organism. Only two transcription factors were regulated at the three times assayed, and others were induced at two times (3), or at one time (15). Assuming a similar number of transcription factors to those described for $S$. cerevisiae, 482 (http://www.yeastgenome.org/), for C. albicans, our results show that this category is underrepresented at $2 \%$ (17 out 783 ) instead of the expected $8 \%$ (482 out of 6033). Two hypothesis can be advanced to account for this observation. The first is that transcription factors act faster in the first 15 minutes of incubation and that after 15 min. most of them have already performed their function. The second one is that the arbitrary limit of $\geq 1.5$-fold variation is too high and that transcription factors act 
with more subtle variations. We are in favour of the second hypothesis, because analysis of the behavior of several transcription factors in a random way indicated that many of them consistently changed outside the imposed limit, i.e. CRZ1 (1.18, 1.16, 1.13); CZF1 (1.22, 1.11, 1.13); EFH1 (1.41, 1.34, 1.02); ROX1 (1.31, 1.22, $0.80)$; $A S H 1(0.80,0.79,0.82)$ or $\operatorname{MIG1}(0.89,0.78,0.69)$ (values at the three times assayed: 15, 60 and $180 \mathrm{~min}$. respectively). We found at least 51 genes corresponding to well-characterized cell wall proteins. Two of the highly induced genes, ALS1 and ALS3, and two repressed genes -ALS2 and ALS4- are all classified in the same subfamily [200]. Functional analyses with RT-PCR and/or by comparison of the behavior of several mutants have been carried out [164,540,541]. A weaker induction was observed in one member of each of the other subfamilies -ALS5 and ALS9- respectively [200]. In our strain, ALS1, ALS5 and ALS9 are in one chromosome [542]. Whether an independent induction or a joint induction occurs must be clarified. Although the genes encoding these proteins share a cross-hybridizing tandem repeat domain, our data suggest that each protein has an different adhesive function, and they are in agreement with those described by Hoyer's group, who show that strains lacking ALS3 or ALS1 do not exhibit a defect in germ tube formation when grown in RPMI 1640 medium, while the als1/als1 mutant formed significantly fewer germ tubes in Lee medium [540]. Our data and those reported previously [164,540,541] point to a different type of regulation for each ALS protein in C. albicans and suggest a role of the ALS genes in cell wall remodelling per se during the yeast-hypha transition, independently of virulence or any other factors, and this is also in agreement with the strong induction of $A L S 1$ during $C$. albicans protoplast regeneration [62].

Upon comparing our results concerning PGA genes with those described by Sentandreu's group [62] on the regeneration/formation of cell wall after $C$. albicans protoplasting, we also observed the up-regulation of PGA6, 13, 54, 56, 59 and 62 (although slightly earlier) and the down-regulation of PGA38. Our only discrepancy concerns SUN41; we found this gene to be up-regulated at the three times of induction, while a down-regulation was described in that work [62].

Another interesting fact is that unlike the up-regulation found in most of the cell wall genes, we observed the down-regulation of 4 putative beta-mannosyltransferases, BMT2, BMT4, BMT5 and BMT6, out of the 9 described for $C$. albicans [321]. This implies that $\beta$-mannosylation is also involved in cell wall remodelling in the yeast- 
hypha transition. It may be predicted that this group of genes, together with those involved in O-mannosylation [274], will be essential for understanding why $C$. albicans is virulent. Several authors have described changes in genes involved in the glycolysis and citric acid and glyoxylate cycles under many different conditions; for example, during phenotypic switching [258], the yeast-hypha transition [342], mutants in APSES proteins [108], and after macrophage internalization [286] or antifungal treatment [281], etc. Our results in general agree with previous ones, but some differences should be noted. Thus, we detected more regulated genes involved in all three pathways than other authors, 38, and most of them were downregulated at $15 \mathrm{~min}$. of incubation at $37^{\circ} \mathrm{C}$ (Figure 2.4). After $180 \mathrm{~min}$. many of the genes had reached a normal level, except 10 that appeared slightly overexpressed. We found a down-regulation of all the genes involved in the citric acid cycle that we were able to detect, while in the APSES mutants these genes always were upregulated. However, genes involved in glycolysis and in reserve carbohydrates were down-regulated in the APSES mutants, as in our experimental conditions [108]. This discrepancy could be due to the different times of incubation or to a direct effect of the efg1 and efh1 APSES mutations, which would directly regulate the genes involved in the citric acid cycle. A switch from glycolysis to gluconeogenesis in phagocytosed $C$.albicans cells has been described [286]. It has also been shown that key enzymes such as aconitase (ACO1), citrate synthase (CIT1), isocitrate lyase (ICL1), malate synthase (MLS1) and malate dehydrogenase $(M D H 1)$ are strongly induced in phagocytosed populations of both $C$. albicans and S. cerevisiae $[134,286,287]$. Our results, however, point in the opposite direction. Thus, we observed a down-regulation of those genes (except ICL1), confirming that the metabolic changes are indeed involved in phagocytosis. Genes involved in carbohydrate metabolism do not seem to be an important group in the response to C. albicans treatment with antifungal agents [281].

We detected an up-regulation of the components of the translation apparatus: 18 genes (up to the $\sim 50$ genes well characterized). The regulation of this group of genes has not been reported in previous works describing the yeast-hypha transition [226,342,427], although up-regulation was found by comparison of a wildtype strain with the efg1, and efh 1 [108] and cwt1 [324] C. albicans mutants. Interestingly, and unlike our results, phagocytosed cells rapidly down-regulate the genes encoding the translation machinery and those coding for a large number of 
transport proteins, including multiple oligopeptide transporters [286]. We detected a decrease in three oligopeptide transporters (OPT1, OPT2 and OPT3) (see Figure 2.6A). Both results are again in agreement with those described in the previous paragraph and support the notion that phagocytosis involves a strong metabolic change.

Many genes related to transport (Table 2.2), oxidases/peorxidases and hydrolases/ dehydrogenases were regulated. Several of them are related to iron transport or to encoding general amino acid permeases. Owing to the large amount of disperse data $[15,24,177,226,255,259,342,376]$ it is difficult to analyze their role, but their prevalence under all kinds of experimental conditions suggests that this group of genes deserves further, more careful analysis in order to establish their specific functions in dimorphism and virulence. The expression of $C D R 1$ and $C D R 2$, which encode multidrug transporters of the $A B C$ family, is up-regulated in the presence of estrogen $[21,91,287]$ and antifungal agents [105,281] and CDR2 is also upregulated in a $C$. albicans double mutant: efg1, efh1 [108]. Our results suggest that the increase in expression of both genes is due only to temperature, and that this (temperature) can act as a kind of pre-adaptation of the Candida machinery to survival in the human host. Our findings also agree with the observed downregulation of CDR4 in the efg1 mutant [108]. Finally, four groups of genes must be mentioned: two of them have been described previously in several works, although not within our experimental context: histone-, and ergosterol-encoding genes. Histone genes are up-regulated in the $C$. albicans efg1 and efg1, efh1 mutants and after exposure to antifungal agents [281]. Ergosterol genes are also mainly upregulated after exposure to antifungal agents [231,281]. No previous data have been reported for the other two sets of genes described in this Chapter; namely, those coding for thiamine and for the $F_{1} F_{0}$ ATPase complex. From our results it may be concluded that the morphogenetic switch involves a strong remodelling of the yeast cell wall and membrane and a change in metabolism. These processes are independent of virulence, although both can be interconnected through an adaptation of $C$. albicans to the host. The data described with both protoplasts and with different sets of mutants are in agreement with our own [62,108,324] and with those obtained from the transcriptional response of $C$. albicans upon internalization by macrophages [286]. The only apparent discrepancy -the different regulation of histone genes- can be explained by a rapid remodelling of chromatin to allow, in 
conjunction with the corresponding transcription factors, the expression of specific hyphal genes, as has been demonstrated by the regulation of efg1 [463], the main transcription factor involved in morphogenesis, by chromatin-remodelling factors. 


\section{Phenotypic, virulence, and transcriptome analysis of the Candida albicans gcn5, hda1 double mutant: Comparison with the gcn5 and hda1 simple mutants}

\section{Abstract}

The fungal pathogen $C$. albicans switches from a yeast-like to a filamentous mode of growth in response to a variety of environmental conditions. Histone acetylases and deacetylases play important roles in eukaryotic gene regulation. Here we deleted the $C$. albicans GCN5 gene in a homozygous hda1 mutant genetic background. We generated a gcn5, hda1 double mutant strain and examined its behavior. The behavior of the double gcn5, hda1 mutant resembled that of the simple gcn5 mutant with respect to growth rate, hyphal outgrowth, chlamydospore formation and sensitivity to Calcofluor white, SDS, Congo red, Hygromycin, Amphotericin B, Fluconazole and Caffeine. Whole-genome microarray analysis of the gcn5, hda1 double mutant revealed an altered expression of 180 (2.98\%) and $664(10.99 \%)$ genes during the yeast mode of growth and during the yeast-hypha transition, respectively. Thirty-nine genes were common to the three times of the yeast-hypha transition assayed (15, 60 and $180 \mathrm{~min}$ ); 35 genes were common between 15 and $60 \mathrm{~min} ; 33$ at 60 and $180 \mathrm{~min}$ and 31 at 15 and $180 \mathrm{~min}$. Of the common up-regulated genes for the three times assayed, 5 genes corresponded to putative transcription factors (BDF1, GAL4, NRG1, TYE7 and ZCF39); 4 to cell wall genes (CRH11, PIR1, RBT2 and RHD3), and 4 to transporter genes (ENA22, GAP5, PMC1 and STL1). Some hypha-specific genes including ALS1, ALS3, ECE1 and HWP1, fatty-acid metabolism-related genes (CYB5, FAD2 and SUR2) and vitamin biosynthesis-related genes ( $\mathrm{BIO} 2$ and $\mathrm{BIO}$ ) were down-regulated during all three times of induction. The expression of other cell wall genes ( $A L S 4, G L C 3$, GSL21, PGA6 and SUN41) was down-regulated at 15 and 60 min of induction. We detected the up-regulation of genes encoding for transcription factors CTA24 (15 and $60 \mathrm{~min}$ ), ZCF1 (60 and $180 \mathrm{~min}$ ), MOT1 and ROX1 (15 and $180 \mathrm{~min}$ ), MDM34 and TOA2 (15 min), RIM101 (60 min), GCN4, SSN6, STP4, TBP1 and ZMS1 (180 min), and down-regulation of HAC1 (15 min), TSM1 and UME6 (60 min) and SPT20 (60 and $180 \mathrm{~min}$ ). Our results include a global transcriptome analysis of the cellular response to the double deletion of the GCN5 and HDA1 genes and afford a basis for a better understanding of the role of this histone acetyltransferase and histone deacetylase in C. albicans. Finally we show that the gcn5 simple mutant is 
avirulent in a mouse model.

\section{Introduction}

The histone acetyltransferase (HAT) Gcn5p plays a pivotal role in chromatin structure and in the regulation of gene expression acting as a catalytic component of multiprotein complexes [64]. In order to facilitate transcription in an activated chromatin environment, the HATs (GCN5) transcription machinery is required for chromatin remodelling and transcriptional activation [374]. Additionally, histone deacetylases (HDACs) remove the acetyl groups of lysine residues of histone tails, leading to chromatin compaction and transcriptional repression [308]. The discovery that a well known transcriptional co-activator, Gcn5p, also has intrinsic HAT activity provided the first direct link between histone acetylation and gene activation [45]. Since then, many studies have confirmed and extended the initial findings in different eukaryotic systems [323,514]. In yeast, Gcn5p is the catalytic component of at least two multi-protein complexes: SAGA and ADA $[117,162]$. Consistent with a role for Gcn5p in the chromatin remodelling of promoters, the recruitment of SAGA by a transcriptional activator leads to histone acetylation and concomitant activation of transcription in vitro [479]. Moreover, the mutation of sequences encoding residues critical for Gcn5p HAT activity in vitro is sufficient to significantly affect the function of the protein in transcriptional activation in vivo [249] and to generate localized alterations in the chromatin structure of target gene promoters [166,250]. Gcn5p and Hda1p are highly conserved among eukaryotes and can acetylate and deacetylate $\mathrm{H} 3, \mathrm{H} 4$ and $\mathrm{H} 2 \mathrm{~B}$ in vitro. Gcn5p and Hda1p might act as a 'chromatin conditioner' in generally establishing and maintaining active chromatin in eukaryotes and in the dynamic maintenance of chromatin organization, respectively. The genome of the yeast Saccharomyces cerevisiae also encodes other HATs, such as Esa1p, Sas2p, Sas3p, Hpa2p, Hat2p and Hat1p $[13,42,237,346,382]$ and HDACs such as Hos1p, Hos2p, Hos3p, Rpd3p and Hda1p. In C. albicans, the gcn5 mutant grows poorly in YEPD rich medium and fails to undergo hypha formation (switching-defective) [106], and the hda1 mutant displays a normal phenotype under both growth conditions [307]. The double mutant displays a phenotype similar to that of the gcn5 mutant. The molecular basis for these growth phenotypes appears to be due to problems with the activation of many genes. In $S$. cerevisiae the gcn5 mutant strain fails to activate 
the HIS4 gene in response to low nitrogen [289], and also shows reduced activation of $\mathrm{HO}$ and some additional genes [248,369]. The somewhat limited phenotypic consequences of the deletion of the transcription-related HATs and HDACs described above could be explained in terms of the existence of functional redundancy. Cellular functions such as transcriptional activation might still proceed almost normally when one HAT complex is non-functional. Similar redundancies could underlie the limited consequence of deleting genes encoding HDACs [396]. Here we suggest that the deletion of GCN5 in double and simple mutants confers typical phenotypes such as slow growth adaptation, defects in yeast-hypha switching, chlamydospore formation, and antifungal sensitivity. In support of the existence of functional overlaps between double and gcn5 mutant strains, we suggest that the acetyltransferase activities of Gcn5p would elicit severe consequences for cell phenotypes. These consequences, however, can also be counteracted by mutating the genes encoding the HDACs: HDA1 and HOS2 [512].

\section{Materials and methods}

\section{Strains and plasmids manipulation}

The Escherichia coli strain used here was grown at $37^{\circ} \mathrm{C}$ in Luria-Bertani medium supplemented with $100 \mu \mathrm{g} / \mathrm{mL}$ of ampicillin for plasmid selection [399].

The C. albicans strains used in this study are listed in Table 3.1. Strains were maintained by periodic transfer to slants of YEPD medium [1\% Yeast extract (Difco), 2\% Bactopeptone (Difco), 1\% Glucose (Difco)]. Yeast growth was obtained in YNB medium at $28^{\circ} \mathrm{C}$ supplemented with $0.2 \mathrm{mM}$ uridine. Solid medium was obtained by adding agar (2\%). Our solid medium for inducing the yeast-hypha transition was Lee medium, in which glucose was replaced by $N$-acetylglucosamine (1.25\%), Spider medium [280], synthetic low-ammonium dextrose medium (SLAHD) $[48,60,270]$ and Embedded medium [43]. 
Table 3.1 Strains used in this study

\begin{tabular}{|c|c|c|c|}
\hline Strain & Genotype & Parental strain & Reference \\
\hline SC5314 & Clinical isolate & & [149] \\
\hline CAI4 & ura3::imm434/ura3::imm434 & CAF2-1 & [132] \\
\hline CAMR & ura3::imm434/URA3 & CAI4 & (Rodriguez and Dominguez, 2005) \\
\hline $\mathrm{NDH} 4$ & $\begin{array}{l}\text { ura3::imm434/ura3::imm434 } \\
\text { hda1::hisG/hda1::hisG }\end{array}$ & CAI4 & (Martin and Dominguez, 2000) \\
\hline SGM1 & $\begin{array}{l}\text { ura3::imm434/ura3::imm434 } \\
\text { hda1::hisG/hda1::hisG } \\
\text { gcn5 hisGURA3hisG/GCN5 }\end{array}$ & $\mathrm{NDH} 4$ & This work \\
\hline SGM2 & $\begin{array}{l}\text { ura3::imm434/ura3::imm434 } \\
\text { hda1::hisG/hda1::hisG } \\
\text { gcn5::hisG/GCN5 }\end{array}$ & SGM1 & This work \\
\hline SGM3 & $\begin{array}{l}\text { ura3::imm434/ura3::imm434 } \\
\text { hda1::hisG/hda1::hisG } \\
\text { gcn5::hisGURA3hisG/gcn5 }\end{array}$ & SGM2 & This work \\
\hline SGM4 & $\begin{array}{l}\text { ura3::imm434/ura3:::imm434 } \\
\text { hda1::hisG/hda1::hisG } \\
\text { gcn5::hisG/gcn5::hisG }\end{array}$ & SGM3 & This Work \\
\hline RAS1 & $\begin{array}{l}\text { ura3::imm434/URA3 } \\
\text { gcn5::hisG/gcn5::hisG }\end{array}$ & RDG4 & This work \\
\hline HAJ1 & $\begin{array}{l}\text { ura3::imm434/URA3 } \\
\text { gcn5::hisG/gcn5::hisG } \\
\text { hda1::hisG/hda1::hisG }\end{array}$ & & \\
\hline RDG4 & $\begin{array}{l}\text { ura3::imm434/ura3::imm434 } \\
\text { gcn5::hisG/gcn5::hisG }\end{array}$ & CAI4 & (Degano and Dominguez, 2000) \\
\hline
\end{tabular}

The dimorphic transition was induced in Lee medium by changing the temperature to $37^{\circ} \mathrm{C}$, by adding $4 \%$ bovine calf serum (GIBCO/BRL), or by changing the carbon source. Uracil prototrophic $\left(\mathrm{Ura}^{+}\right)$transformants were further subjected to 5fluoroorotic acid (5-Foa; Sigma) selection for excision of the URA3 marker [132]. Strains for generation time assays were grown on rich medium (YEPD) at an O. $D_{600 \mathrm{~nm}}$ of 0.1 , and growth was monitored over a period of $34 \mathrm{~h}$ by measuring the optical density of the cultures. To induce hyphal development on solid media, $C$. albicans cells were grown overnight at $28^{\circ} \mathrm{C}$ in YEPD liquid medium and washed twice with water before being plated. Photomicrographs of colonies and invasive growth were taken with a DMRXA microscope (Leica, Germany). To observe chlamydospore formation, a CAl4 strain -CAl4, an isogenic series of C. albicans strain derived from SC5314- and the double mutant were streaked out lightly on cornmeal agar (Difco)-0.33\% Tween 80, coverslipped, and incubated at $25^{\circ} \mathrm{C}$ for 14 days. 


\section{DNA manipulation}

Total DNA from C. albicans was prepared as described previously for filamentous fungi $[399,400]$. Restriction enzyme digestions and DNA ligations were performed according to the recommendations of the manufacturers. Isolation of plasmid DNA from E. coli was performed by standard procedures [399]. C. albicans cells were transformed using the spheroplast protocol [391]. Oligonucleotides were supplied by Roche (www.roche-as.es). A list of the primers used can be found in Table 3.2. A southern analysis was performed using a radioactive procedure. DNA fragments used as probes were labelled by the Rediprime ${ }^{T M}$ II Random Prime labelling system from Amersham-Biosciences with $\left(\alpha-{ }^{32} \mathrm{P}\right) \mathrm{dCTP}$ (Boehringer Mannheim) and were used according to the manufacturer's instructions.

Table 3.2 Primers used in this study

\begin{tabular}{|c|c|c|c|}
\hline Primer & Sequence $5^{\prime}-3$ & Primer & Sequence $5^{\prime}-3^{\prime}$ \\
\hline $\begin{array}{l}\text { RD56 } \\
\text { RD57 } \\
\text { RD64 } \\
\text { RD54 } \\
\text { RD55 } \\
\text { URA2 } \\
\text { AHM1 } \\
\text { GCN5-Outer }\end{array}$ & $\begin{array}{l}\text { TAT CCA TTG TCC AGG TGG TT } \\
\text { ACT GAA GTA GAC TTC CCA CT } \\
\text { TTC AGT TGA CGA GAT AGC AC } \\
\text { ATT GAA AGA CTG CAG TTT TGT } \\
\text { CTT ATT CTC GAG CAA TGC TT } \\
\text { AAT GCT GGT TGG AAT GCT TA } \\
\text { CCG AAC ATC AAC CAA TCG T } \\
\text { ACG GTT GTT AAA TGG ACG GTA }\end{array}$ & $\begin{array}{l}\text { URA1 } \\
\text { AHM IP2 } \\
\text { AHM IP3 } \\
\text { GCN5-Inner } \\
\text { 5' RACE-Inner } \\
\text { 5' RACE-Outer }\end{array}$ & $\begin{array}{l}\text { GGA TAC TAT CAA ACA AGA GG } \\
\text { TTA CAA TCA AAG GTG GTC C } \\
\text { GGT ACA GTT CCT CAC ATC } \\
\text { CTT TCT CAC AAC TGC CAT TGA } \\
\text { GCG GGA TCC GAA CAC TGC GTT } \\
\text { TGC TGG CTT TGA TG } \\
\text { GCT GAT GGC ATG AAT GAA } \\
\text { CACTG }\end{array}$ \\
\hline
\end{tabular}

\section{Chromosomal disruption of the GCN5 gene}

Deletion of GCN5 in the hda1 background strain was achieved by the "Ura-blaster" procedure [132]. Plasmid pCJR3 was used to generate the double mutant strain. This plasmid was constructed by Degano at our laboratory. Strain NDH4 was transformed by the protoplast method. Resistance to 5-fluoroorotic acid led to spontaneous loss of the hisG-URA3 cassette. Deletion of the gene and loss of the URA3 gene was confirmed by Southern blot analysis.

\section{RLM-RACE}

The $C$. albicans transcript was mapped using the FirstChoice RLM-RACE kit (Ambion). RNA was isolated from CAI4 at exponential time by breaking the cells in a micro-dismembrator (Braun, Melsungen), followed by the trizol extraction method as described in Galar Fungal standard operating procedures for RNA extraction. Total RNA was treated with Calf Intestinal alkaline Phosphatase (CIP) to remove 
the 5'-phosphate from non-full-length uncapped RNA. The 5' cap was removed from full-length mRNA by treatment with Tobacco Acid Pyrophosphatase (TAP), leaving a 5'-monophosphate to which a 5' RACE adapter $(0.3 \mu \mathrm{g} / \mathrm{ml})$ oligonucleotide (5'GCUGAUGGCGAUGAAUGAACACUGCGUUUGCUGGCUUUGAUGAAA 3') was ligated using T4 RNA ligase. Random-primed reverse transcription and nested PCR were used to amplify the 5' transcript. The outer PCR reaction used the 5' RACE outer primers, whereas the inner PCR reaction used the 5' RACE inner primers (Table 3.2). The 5' RACE inner PCR products were cloned into pGEM-T (Promega) and sequenced.

$\mathrm{NaCl}$, Calcofluor white, Caffeine, SDS, Fluconazole, Itraconazole, Amphotericin B and Hygromycin sensitivities

The methods used to test the $C$. albicans strains were similar for all the effectors. Cultures were grown in $50 \mathrm{ml}$ of YEPD medium with $1 \%$ glucose until the exponential phase and diluted to an $0 . D_{600 \mathrm{~nm}}$ of 0.4 . Five microlitres of pure and $1 / 10$ serial dilutions of each cell culture were spotted onto YEPD plates containing $\mathrm{NaCl}(0.5$ to $1 \mathrm{M})$, Calcofluor white $(0.05$ to $1 \mathrm{mg} / \mathrm{ml})$, Caffeine $(15 \mathrm{mM})$, SDS $(0.005 \%$ to $0.05 \%)$, Fluconazole $(4 \mu \mathrm{g} / \mathrm{ml})$, Amphotericin B (50 to $100 \mu \mathrm{g} / \mathrm{ml}$ ), Hygromycin (100 to $300 \mu \mathrm{g} / \mathrm{ml}$ ) and Congo red $(0.1$ to $0.5 \mathrm{mg} / \mathrm{ml})$. Differences in growth were recorded after incubation of the plates at $28^{\circ} \mathrm{C}$ for $72 \mathrm{~h}$.

\section{DNA sequencing and sequence analysis}

Both strands of the PCR product described above were sequenced using the DNA Sequencing Service of the University of Salamanca with an Applied Biosystems ABI PRISM 3100 Genetic Analyzer.

\section{Zymolyase sensitivity phenotypic test}

Cultures of the CAl4, gcn5, hda1 and both simple mutant strains were grown in YEPD medium up to the exponential phase. Cells were washed twice in water and resuspended in $10 \mathrm{mM}$ Tris- $\mathrm{HCl}\left(\mathrm{pH} \mathrm{7.5)}\right.$ and $0.3 \% \beta$-mercaptoethanol. $2 \times 10^{7}$ cells were resuspended in the same buffer containing 20T Zymolyase at a concentration of $0.01 \mathrm{mg} / \mathrm{ml}$. The optical density at $600_{\mathrm{nm}}$ was measured at the start of the incubation and every $20 \mathrm{~min}$ thereafter. The decrease in optical density reflected the portion of cells that had lysed. 


\section{Alcian blue binding assay}

The Alcian blue binding assay was carried out using the method of Herrero et al [189]. A series of solutions containing different amounts of Alcian blue were prepared in $0.02 \mathrm{~N} \mathrm{HCl}$ and the optical density at $600_{\mathrm{nm}}$ of each solution was determined. A standard curve was plotted of the O.D values versus the amounts of Alcian blue. To quantify Alcian blue binding to the cell surface, an aliquot of $1000 \mu \mathrm{l}$ of exponential-phase yeast cells $\left(O . D_{600 \mathrm{~nm}}, 5\right)$ was centrifuged, and the cells were washed twice with $1 \mathrm{ml}$ of $0.02 \mathrm{~N} \mathrm{HCl}$ and resuspended in $1 \mathrm{ml} 0.02 \mathrm{~N} \mathrm{HCl}$ containing $100 \mu \mathrm{g}$ Alcian blue. The cell suspension was allowed to stand for $10 \mathrm{~min}$ at room temperature and then centrifuged for $3 \mathrm{~min}$ to pellet the cells. Then, the O. $D_{600 \mathrm{~nm}}$ of the supernatant was measured. The amount of dye bound to the cells was calculated by subtracting the amount of dye in the supernatant from $100 \mu \mathrm{g}$.

\section{RNA isolation, cDNA preparation and microarray hybridization}

C. albicans CAI4 and SGM4 strains were grown in YNB [0.67\% Yeast Nitrogen Base without amino acids (YNB w/o aa, Difco), 1\% Glucose (Difco)] and Lee for yeast and hypha growth conditions, respectively. RNA isolation, cDNA preparation and microarray hybridization were carried out as in the previous chapter II.

\section{Results}

\section{Determination of transcription initiation sites using RNA ligase-mediated Rapid Amplification of 5' cDNA Ends (5' RLM-RACE) of GCN5 gene}

To identify transcription initiation sites, RLM-RACE was performed. The results are shown in Figure 3.1. We amplified one band of $\sim 500 \mathrm{bp}$, which was cloned in pGEM-T. After sequencing 5 different clones, three putative transcription start sites (three identical and two different) were identified. These products may have originated from different transcription start sites. The three positions 22,32 and 39 bp were taken as the transcription start site (Figure 3.1). In any case, the GCN5 transcript is relatively short only a few bp upstream from the ATG codon. It should be noted that very few data about the length of transcripts are available for $C$. albicans. 


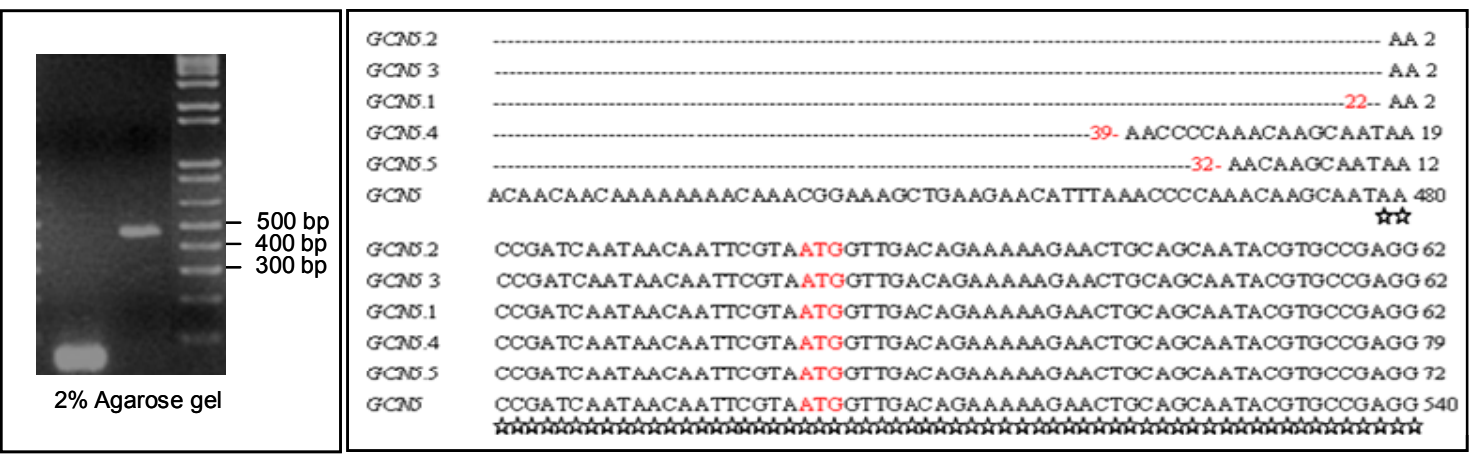

Figure 3.1 Mapping of GCN5 transcription initiation sites by RLM-RACE. Predicted transcription start site of the GCN5 gene

\section{Disruption of GCN5 in an hda1 mutant background strain}

We deleted the ORF of the GCN5 gene with the "Ura-blaster" method [132] using protoplasts [391] in a hda1 background strain. Deletion was verified by using two pairs of primers for PCR analysis (data not shown) and by Southern blot analysis (Figure 3.2). With the double mutant we performed a whole set of experiments and below we describe the results obtained.

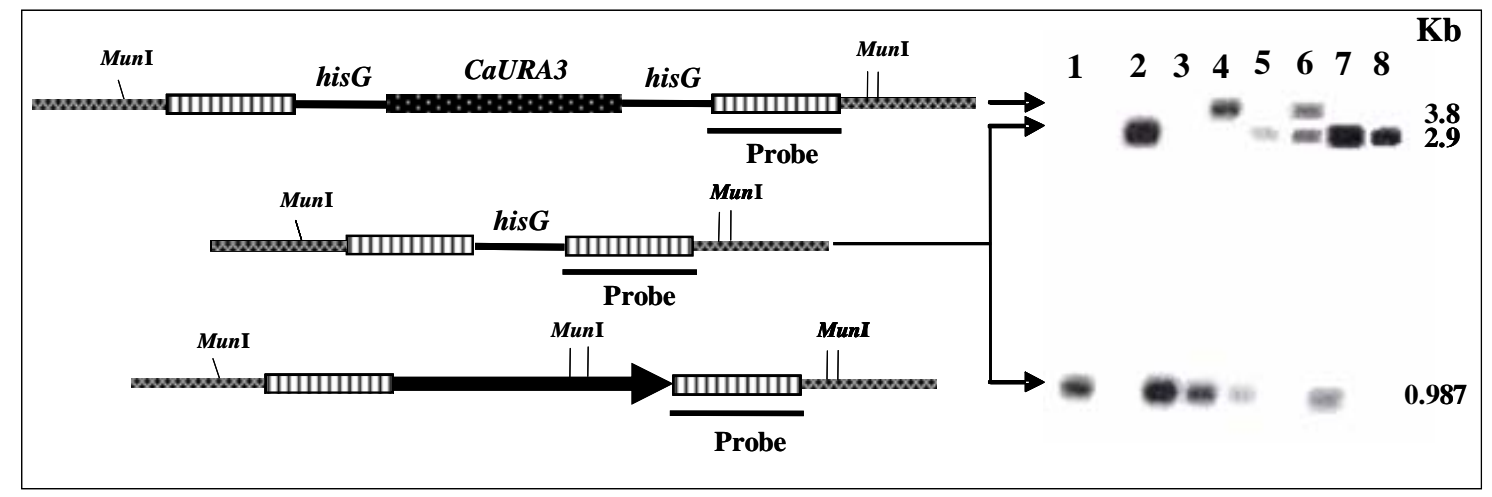

Figure 3.2 Southern blot analysis of the SGM4 double mutant gcn5, hda1 using the Rsal/EcoRI fragment of the GCN5 gene as probe. Genomic DNA samples were digested with Munl. Lane 1, CAl4 (ura3/ura3 GCN5/GCN5).

Lane 2, RDG4 (ura3/ura3 gcn5/gcn5::hisG). Lane 3, NDH4 (ura3/ura3 hda1/hda1::hisG)

Lane 4, SGM1 (ura3::imm434/ura3::imm434hda1::hisG/hda1::hisG gcn5::hisGURA3hisG /GCN5)

Lane 5, SGM2 (ura3::imm434/ura3::imm434hda1::hisG/hda1::hisGgcn5::hisG/GCN5)

Lane 6, SGM3 (ura3/ura3 gen5::hisG-URA3-hisG/gcn5::hisG)

Lane 7, SGM4-3 (ura3::imm434/ura3::imm434 hda1::hisG/hda1::hisG gcn5::hisG/gcn5::hisG)

Lane 8, SGM4-11 (ura3:::imm434/ura3:::imm434 hda1:::hisG/hda1::hisGgcn5::hisG/gcn5::hisG).

\section{The gcn5, hda1 double mutant has a growth defect}

To analyze the growth rate of the double mutant we performed a comparison of growth between all four strains: CAI4 (GCN5, HDA1), NDH4 (hda1), RDG4 (gcn5) and SGM4 (gcn5, hda1) (Figure 3.3). The beginning of the exponential growth phase in the double mutant, as in the case of the gcn5 simple mutant, was delayed 
by up to $22 \mathrm{~h}$. At our laboratory Martin [307] failed to detect any significant difference in growth rate, cell size or morphology between the hda1 mutant and the CAI4 strain. Furthermore, the homozygous mutant of gcn5 [106] shows a delay in the entry into the logarithmic phase (Figure 3.3).

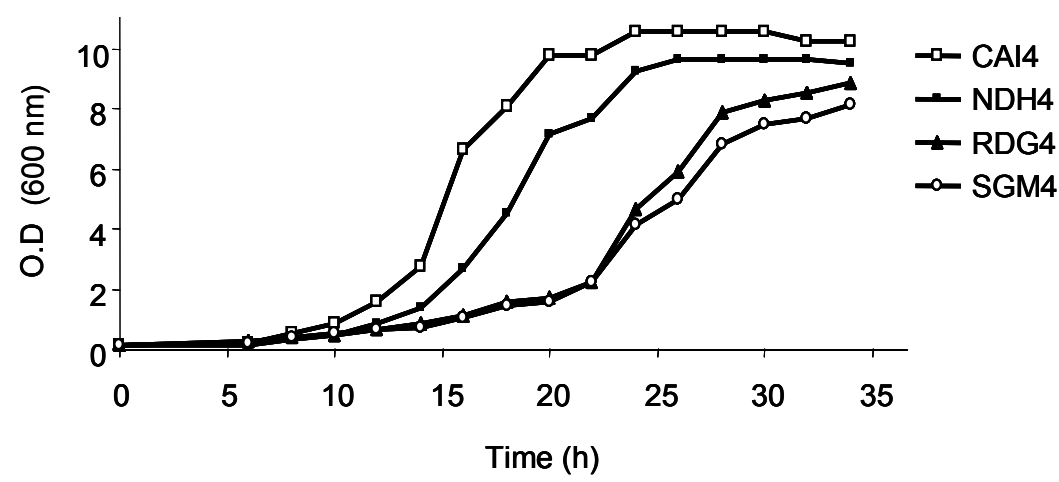

Figure 3.3 Growth curves of CAI4, NDH4 (hda1), RDG4 (gcn5) and SGM4 (gcn5, hda1). Strains were pre-grown on rich medium containing $1 \%$ glucose and inoculated at an O.D at $600_{\mathrm{nm}}(0.1)$ YEPD. Growth was monitored every two hours over a period of $34 \mathrm{~h}$ by measuring the optical density of the cultures.

These results suggest that GCN5 would be involved in vegetative growth in $C$. albicans and that HDA1 would be unable to compensate this effect. Morphologically, the double mutant, like the gcn5 simple mutant, is very different to the CAl4 strain, as shown in Figure 3.4. The culture of the gcn5, hda1 double mutant in YEPD liquid medium was composed of a mixture of spherical cells and short chains of elongated, aberrantly shaped cells attached to one another (Figure 3.4B), suggesting that Gcn5p activates or suppresses the transcription of genes that directly or indirectly control cell morphology.

CA14

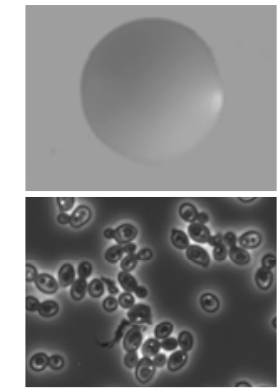

SGM4

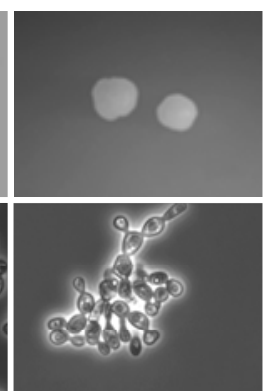

Figure 3.4 (A) Colony growth and (B) morphological characteristics of C. albicans homozygous strain CAI4 and double mutant gcn5, hda1 in YEPD medium.

\section{Behavior of the double mutant gcn5, hda1 in solid media}

For the observation of filamentous growth on solid media, cells of the simple and 
double mutants as well as the CAI4 strain were plated at 50 ufc on plates. Solid media normally induce filamentous growth after 3 days. The CAI4 strain displayed normal filamentous growth, with long chains of hypha growing outwards from the colony (Figure 3.5). In contrast, the gcn5, hda1 mutant was defective for filamentous growth in all media assayed. The colonies had completely smooth edges and all cells examined were in the budding yeast form (data not shown). These results indicated that both the GCN5 and HDA1 genes were necessary for efficient filamentation on solid media. We also observed that, like both simple mutants, the double mutant was unable to form hypha in Embedded medium (Figure 3.5).

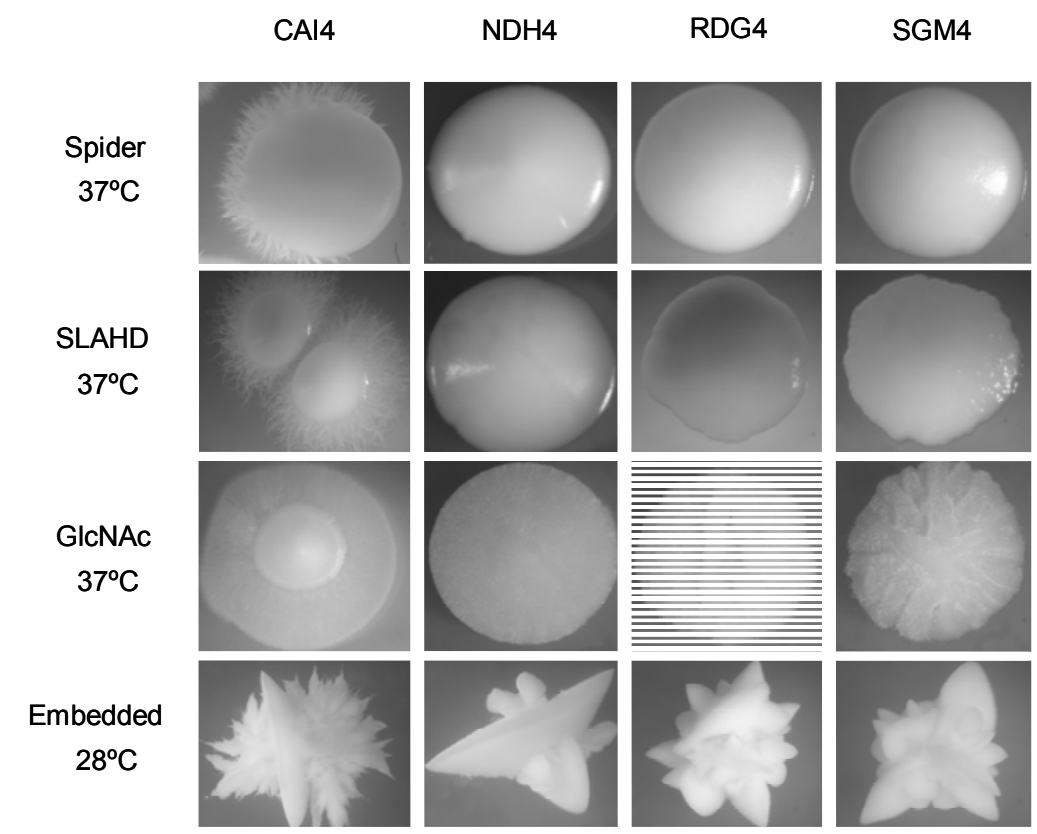

Figure 3.5 Phenotypes of the CAI4, NDH4 (hda1), RDG4 (gcn5) and SGM4 (gcn5, had1) on solid media. Plates were incubated for 5 days at $37^{\circ} \mathrm{C}$.

\section{Behavior of the gcn5, hda1 mutant in liquid media}

The behavior of the double mutant strain was next investigated by assessing hyphal formation in Lee $\left(\mathrm{pH} 6.8,37^{\circ} \mathrm{C}\right)$ or in serum inducing media. Figure 3.6 shows that the double gcn5, hda1 mutant failed to form germ tubes in any of the liquid media assayed. 
A.

CAI4

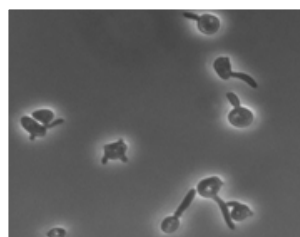

$4 \mathrm{~h}$

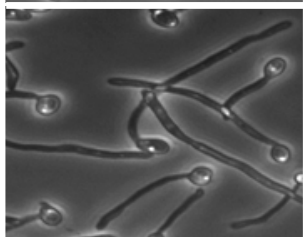

Lee $+4 \%$ Serum, $37^{\circ} \mathrm{C}$
B.

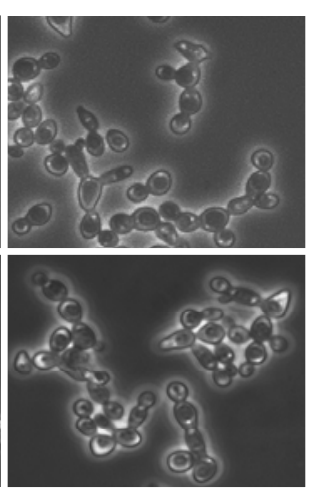

$1 \mathrm{~h}$

$4 \mathrm{~h}$

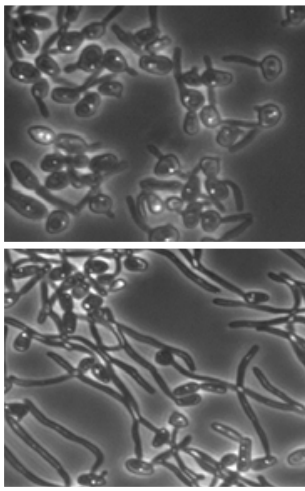

Lee, $37^{\circ} \mathrm{C}$

SGM4

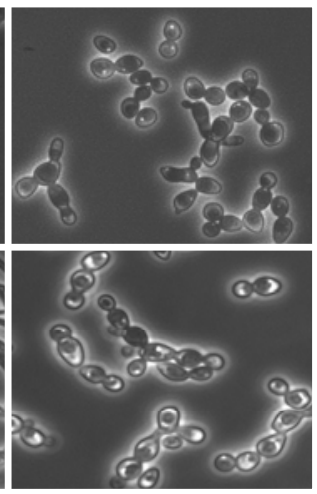

ypha transition in Lee + $4 \%$ serum $(A)$ and Lee medium $(B)$ at $37^{\circ} \mathrm{C}$.

\section{Chlamydospore formation}

We attempted to determine whether the deletion of both genes might be involved in the formation of chlamydospores. The results are shown in Figure 3.7. The gcn5, hda1 double mutant, like the gcn5 simple mutant, was completely deficient in chlamydospore formation. In contrast, hda1 [307] and the CAl4 strain were able to form chlamydospores under the same conditions. These results confirm that Gcn5p is required for the activation of the expression of chlamydospore formation-related genes and again demonstrate that there is no compensatory effect on Hda1p.
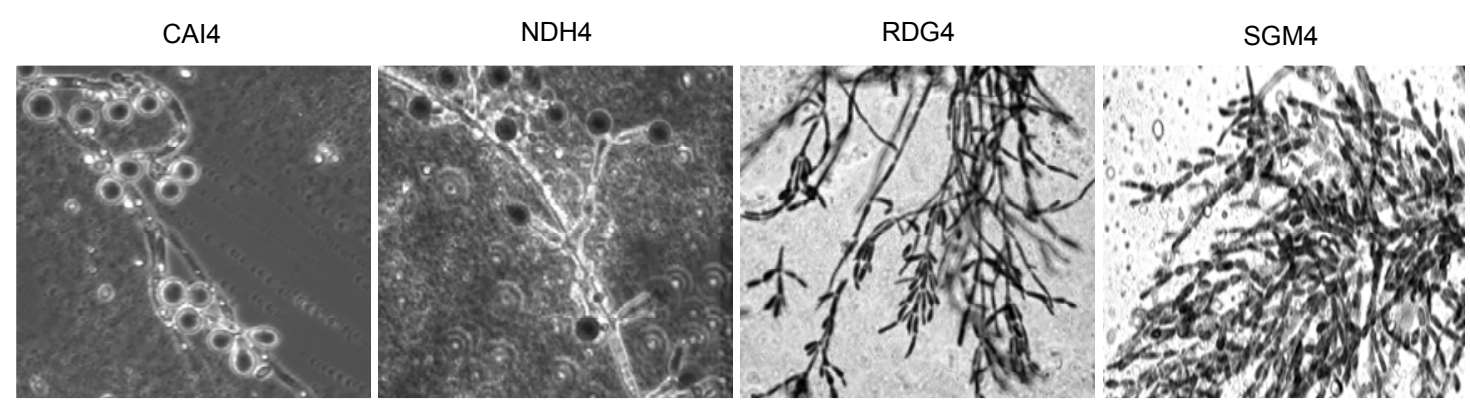

Figure 3.7 Chlamydospore formation in C. albicans CAI4, NDH4 (hda1), RDG4 (gcn5) and SGM4 (gcn5, hda1) mutant.. The strains were lightly streaked out on chlamydospore induction medium (cornmeal agar (Difco)-0.33\% Tween 80 and incubated at $25^{\circ} \mathrm{C}$ for 14 days.

\section{Sensitivity to CFW, Congo red, $\mathrm{NaCl}$ and SDS}

Using a screening method designed to identify genes involved in cell surface assembly [293], we found that the gcn5 and gcn5, hda1 double mutant showed a moderate sensitivity to Calcofluor white and $\mathrm{NaCl}$, and hypersensitivity to Congo red and SDS; the results are shown in Figure 3.8. These findings prompted us to 
carry out a further six phenotypic tests (Hygromycin, Amphotericin B, Fluconazole, Caffeine and Zymolyase sensitivity) in order to bether characterize and define the gcn5, hda1 simple and double mutants. The gcn5 and the gcn5, hda1 double mutant were hypersensitive to Hygromycin, Amphotericin B, Fluconazole and Caffeine (Figure 3.8). The growth of the hda1 mutant and CAI4 was not affected at the same concentrations.
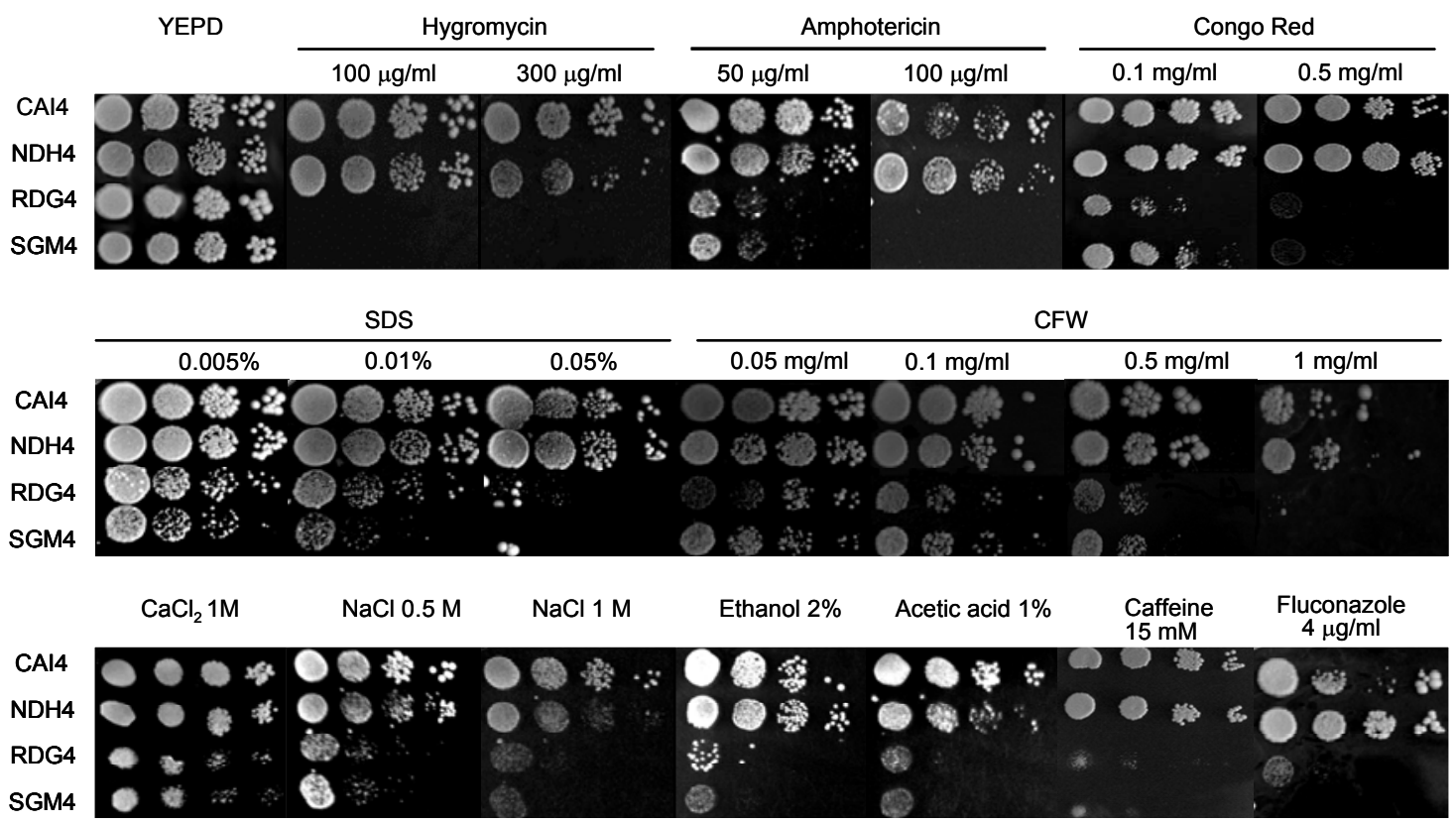

Figure 3.8 Sensitivity assays of CAI4, NDH4 (hda1), RDG4 (gcn5) and SGM4 (gcn5, hda1) to different agents. All strains were grown in liquid YEPD. The optical density at $600 \mathrm{~nm}$ of the cultures was adjusted to 0.4 with the same medium, and $5 \mu \mathrm{l}$ aliquots from the cultures and from 10 -fold serial dilutions was spotted onto YEPD plates supplemented with different agents. Plates were incubated at $28^{\circ} \mathrm{C}$ for 4 days.

The sensitivity of yeast cells to Zymolyase has been used to detect changes in cell wall composition and arrangement [375]. Our results indicated that there were no significant differences between the CAI4 and any of the mutant strains as regards sensitivity to Zymolyase (Figure 3.9).

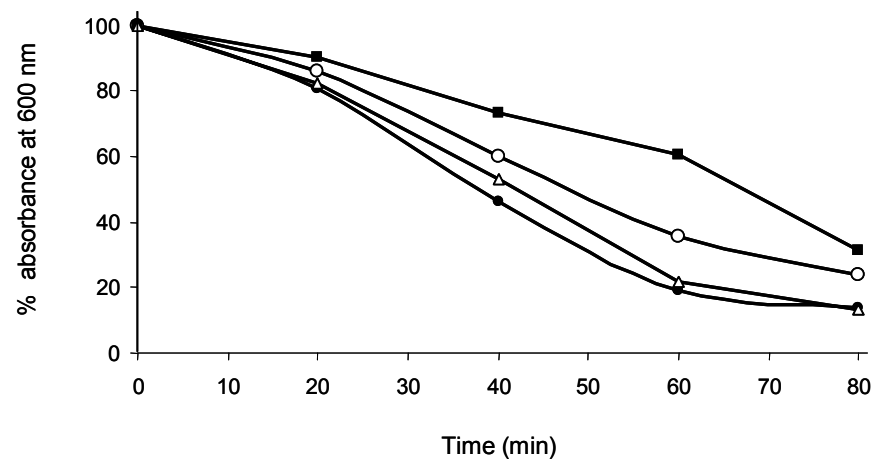

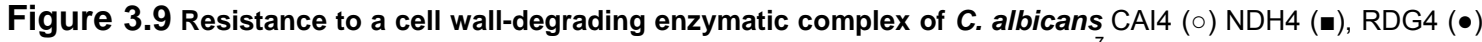
and SGM4 $(\Delta)$ were grown up to the exponential phase in YEPD at $28^{\circ} \mathrm{C}$. Aliquots $\left(2 \times 10^{7}\right.$ cells) were resuspended in 10 $\mathrm{mM}$ Tris- $\mathrm{HCl}, \mathrm{pH} 7.5$, and treated with $10 \mu \mathrm{g}$ of Zymolyase per ml. The decrease in optical density (O.D) (percentage of resistant cells) was monitored as described in Methods. 


\section{Alcian blue binding assay}

We then determined the level of Alcian blue binding of CAl4 and the three mutant strains of $C$. albicans, using the method previously described [189]. All three mutants showed a significant increase in Alcian blue binding, suggesting a strong acidification of the cell wall composition (Figure 3.10). In this case, an additive effect was detected. Thus, the gcn5, hda1 double mutant increased binding by $85 \%$, which roughly corresponded to the addition of the increase in Alcian blue binding of the simple mutants: $h d a 1,20 \%$, and gcn5, $55 \%$.

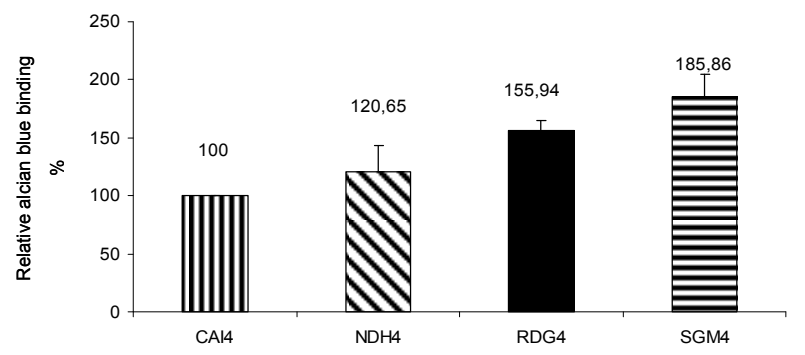

Figure 3.10 Alcian blue binding assays. Relative dye binding was calculated as the percentage of dye bound compared with results obtained for the parental strain CAI4

\section{The GCN5 gene is required for $C$. albicans virulence}

Our observation that the $C$. albicans GCN5 gene was required for hyphal emergence and/or elongation in vitro, led us to examine whether GCN5 might also be required for $C$. albicans virulence. Mice were injected intra-peritoneally with a reconstructed URA3 gcn5 and the double mutant strain and survival was monitored over a period of 30 days. Two days after infection, all the mice injected with the CAMR strain showed signs of systemic disease, including weight loss (data not show) and rapid mortality. Twenty percent of the mice injected with gcn5, hda1 double mutant cells survived. No difference in mortility was found between mice infected with the hda1 [307] and the CAMR strains. In contrast, $80 \%$ of the mice injected with $5 \times 10^{8}$ cells of the gcn 5 null mutant survived for the observational period of 30 days (Figure 3.11). 


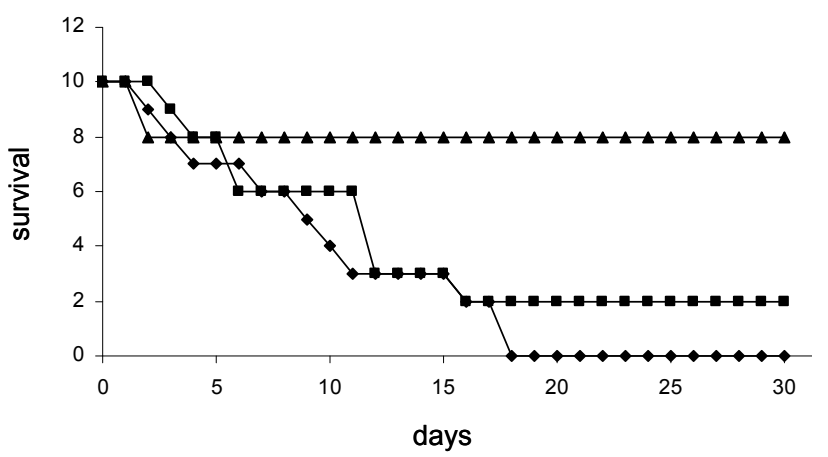

Figure 3.11 Virulence assays. Survival curves for mice $(n=10$ for each $C$. albicans strain at each inoculation dose) infected with $5 \times 10^{8}$ cells of C. albicans. CAMR (CAl4-URA ${ }^{+}$), \ RAS1 (gcn5/gcn5-URA $\left.{ }^{+}\right)$, HAJI1 (gcn5, hda1$\left.U R A^{+}\right)$.

\section{Genome-wide analysis}

Transcript profiling was used to further examine the role of the Gcn5p and Hda1p proteins in C. albicans. The transcriptome of the gcn5, hda1 double mutant was compared with the CAI4 strain during the yeast mode of growth and during the yeast-hypha transition. Previously, at our laboratory we had performed experiments in which the transcriptomes of the gcn5 and hda1 simple mutants were analyzed. Genes that displayed reproductive and statistically significant changes in expression were identified under both yeast growth conditions and during the yeast-hypha switch. A list of differentially expressed genes in the double mutant is provided as Supplementary data (Supplementary Tables 3.1 and 3.2). To explore the functions of the gcn5, hda1 double mutant under conditions that promoted growth in the yeast form, we compared the transcriptomes of CAI4 and the double mutant strain in YNB medium at $28^{\circ} \mathrm{C}$. In the gcn5, hda1 double mutant we detected an altered expression of 180 genes by $\geq 1.5$-fold: 84 genes being upregulated and 96 being down-regulated.

Only eight genes were affected by the deletion of the HDA1 gene (all genes were down-regulated) (Figure 3.12). Three genes -ALS2, ALS4 and BGL22- were commonly affected in both the double and simple mutants. The simultaneous deletion of GCN5 and HDA1 showed a transcriptional pattern that mainly overlapped with that of the gcn5 single mutant (47 genes) (Figure 3.12). 


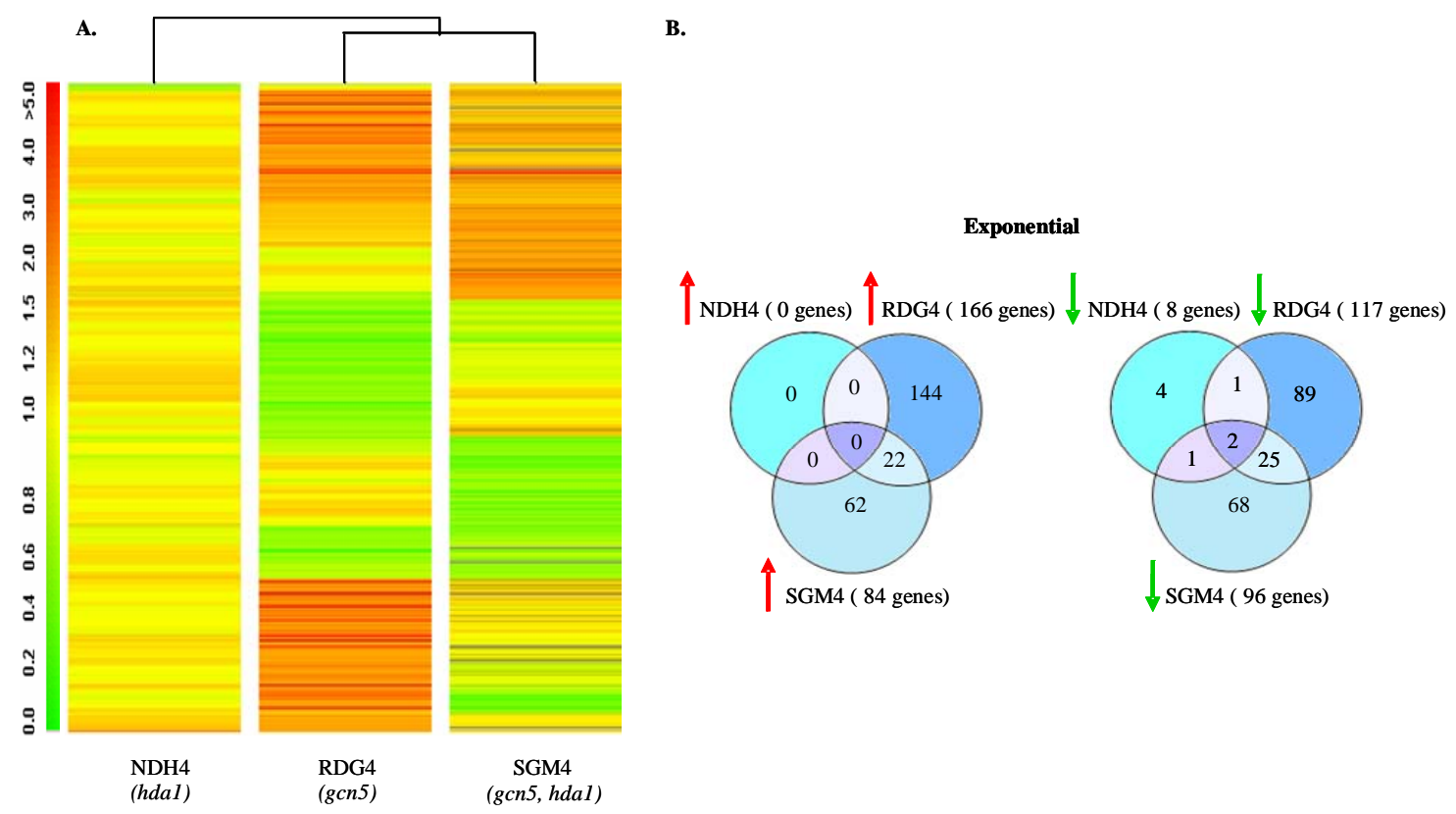

Figure 3.12 A. Transcript profiles of the $C$. albicans gcn5 and hda1 single and double mutants during the yeast growth form (A). Venn diagrams of genes regulated by Gcn5p and Hda1p in the single and double mutant strains. The CAl4 strain was used as a reference for the gcn5 mutant (RDG4), the hda1 mutant (NDH4) and the gcn5, hda1 double mutant (SGM4), which were all grown under identical conditions: $\mathrm{YNB} / 28^{\circ} \mathrm{C}$ (non-inducing) (B).

However, a subset of 130 genes was affected in the double mutant that was not altered in either of the simple mutants. These results indicate that under standard growth conditions both genes jointly regulate an important set of genes.

Categorization of the regulated genes according to their functional categories of the MIPS database (http://mips.gsf.de/genre/proj/yeast/searchCatalogFirstAction. do? db_CYGD) revealed that double deletion of the GCN5 and HDA1 genes had a striking effect on carbon metabolism. The transcript levels of almost all the glycolytic genes (e.g., FBA1, PFK1 and PFK2) and of the genes involved in the metabolism of the reserve sugars trehalose and glycogen (e.g., TPS2 and GSY1) were reduced in the double mutant by a factor of 1.5 (Table 3.2). In addition, the transcript levels of the ACO1 (aconitate hydratase), LSC2 (succinate-CoA ligase, beta subunit) and TAL1 (transaldolase) were increased (Supplementary Table 3.1). 
Table 3.3 Differentially expressed genes in the gcn5, hda1 mutant during the yeast mode of growth

\begin{tabular}{lllc}
\hline Gene name & Accession no & Description & Fold regulation \\
\hline Up-regulated genes & & EXP \\
Cell wall & & & \\
AAF1 & orf19.7436 & Adhesion and aggregation mediating surface antigen & 1.68 \\
CSP37 & orf19.2531 & Plasma membrane hyphal cell wall protein & 1.66 \\
PGA13 & orf19.6420 & Putative GPI-anchored protein & 1.57 \\
PGA56 & orf19.1105.2 & Regulator of sorbose utilization; putative GPI-anchor & 1.73 \\
Transcription & & & \\
RPD32 & orf19.6801 & Histone deacetylase B & 1.59 \\
ZCF32 & orf19.5940 & Putative transcriptional regulator & 1.80
\end{tabular}

\section{Down-regulated genes}

Glycolysis and gluconeogenesis

$\begin{array}{ll}\text { ADH1 } & \text { orf19.3997 } \\ \text { ADH5 } & \text { orf19.2608 } \\ \text { CDC19 } & \text { orf19.3575 } \\ \text { FBA1 } & \text { orf19.4618 } \\ \text { GPM2 } & \text { orf19.1067 } \\ \text { HXK2 } & \text { orf19.8176 } \\ \text { PDC11 } & \text { orf19.2877 } \\ \text { PFK1 } & \text { orf19.3967 } \\ \text { PFK2 } & \text { orf19.6540 } \\ \text { PG11 } & \text { orf19.3888 } \\ \text { PGK1 } & \text { orf19.3651 } \\ \text { PYC2 } & \text { orf19.789 } \\ \text { TDH3 } & \text { orf19.6814 } \\ \text { TPI1 } & \text { orf19.6745 }\end{array}$

Amino acid metabolism

$\begin{array}{ll}\text { ARO8 } & \text { orf19.2098 } \\ \text { BAT22 } & \text { orf19.6994 } \\ \text { ECM17 } & \text { orf19.4099 } \\ \text { HOM3 } & \text { orf19.1235 } \\ \text { LYS12 } & \text { orf19.2525 } \\ \text { MET6 } & \text { orf19.10083 } \\ \text { MIS11 } & \text { orf19.2364 } \\ \text { SAM2 } & \text { orf19.657 }\end{array}$

Drug resistance

$\begin{array}{ll}\text { CDR1 } & \text { orf19.6000 } \\ \text { CDR4 } & \text { orf19.5079 }\end{array}$

Ferroxidase and ion transport

$\begin{array}{ll}\text { FET99 } & \text { orf19.4212 } \\ \text { FET34 } & \text { orf19.4215 } \\ \text { FTR1 } & \text { orf19.7219 }\end{array}$

Alcohol dehydrogenase I

Probable alcohol dehydrogenase

Pyruvate kinase

Fructose-bisphosphate aldolase

Phosphoglycerate mutase

0.30

Hexokinase II

0.48

Pyruvate decarboxylase

6-phosphofructokinase, Alpha subunit

0.39

0.35

0.31

6-phosphofructokinase, Beta subunit 0.23

Glucose-6-phosphate isomerase $\quad 0.63$

$\begin{array}{ll}\text { Phosphoglycerate kinase } & 0.47\end{array}$

Pyruvate carboxylase $2 \quad 0.54$

Glyceraldehyde-3-phosphate dehydrogenase $\quad 0.35$

Triose phosphate isomerase

Aromatic amino acid aminotransferase I $\quad 0.61$

Branched chain amino acid aminotransferase $\quad 0.65$

$\begin{array}{ll}\text { Putative sulfite reductase } & 0.62\end{array}$

Aspartokinase 0.68

Homo-isocitrate dehydrogenase $\quad 0.65$

Methyltetrahydropteroyltriglutamate $\quad 0.60$

Mitochondrial C1-tetrahydrofolate synthase $\quad 0.55$

S-adenosylmethionine synthetase $2 \quad 0.47$

Multidrug resistance protein $\quad 0.65$

Multidrug resistance protein $\quad 0.66$

Cell surface ferroxidase $\quad 0.60$

Multicopy oxidase $\quad 0.66$

High affinity iron permease $\quad 0.62$

Lipid, Fatty-acid and isoprenoid metabolism

$\begin{array}{llll}\text { FAS2 } & \text { orf19.5951 } & \text { Fatty-acyl-coa synthase, Alpha chain } & 0.50 \\ \text { ERG1 } & \text { orf19.406 } & \text { Squalene epoxidase } & 0.44 \\ \text { SHM2 } & \text { orf19.5750 } & \text { Serine hydroxymethyltransferase precursor } & 0.66 \\ \text { SUR2 } & \text { orf19.5818 } & \text { Hydroxylation of C-4 of the sphingoid moiety of ceramide } & 0.36\end{array}$

Metabolism of energy reserves (glycogen. Trehalose)

$\begin{array}{lll}\text { GSY1 } & \text { orf19.3278 } & 0.55\end{array}$

TPS2 orf19.3038

Threalose-6-phosphate phosphatise

$60 S$ ribosomal protein

0.66

Ribosomal protein L10. Cytosolic 0.58

$60 \mathrm{~S}$ ribosomal protein 17 a.e.B $\quad 0.51$

60S ribosomal protein 17 a.e.B $\quad 0.67$

Acidic ribosomal protein S12 0.63

40S ribosomal protein S15 0.49

Ribosomal protein S19.e 0.54

Ribosomal protein s15a.e.c10 $\quad 0.66$ 


\begin{tabular}{llll} 
RPS7A & orf19.9267 & Ribosomal protein & 0.67 \\
RPS8A & orf19.6873 & Ribosomal protein & 0.56 \\
YST1 & orf19.6975 & Ribosomal protein & 0.57 \\
\multicolumn{2}{l}{ Transcription factor } & & \\
EFG1 & orf19.8243 & Enhanced filamentous growth factor & 0.68 \\
TYE7 & orf19.4941 & Basic helix-loop-helix transcription factor & 0.69 \\
UPC2 & orf19.391 & Similar to S. cerevisiae Upc2p & 0.63 \\
Transport & & & 0.66 \\
ATP5 & orf19.5419 & F1F0-ATPase complex & 0.29 \\
HXT62 & orf19.2023 & Sugar transporter & 0.29 \\
\hline
\end{tabular}

Description as Candida Genome date bases

In our experiments, we observed the down-regulation of EFG1. It has been reported that the transcript levels of almost all glycolytic genes and of genes involved in the metabolism of the reserve sugars trehalose and glycogen are reduced in an efg1 mutant [108]. Our result also suggested that there is a indirect control on the expression of glycolytic genes in double mutant cells. We found 3 genes (CSP37, PGA13 and PGA56), encoding for cell wall and cell surface proteins, to be up-regulated in the double mutant. CSP37 is a membraneassociated protein and the deletion of both alleles of the gene showed no apparent defect in cell viability, growth, or cell wall assembly but displayed attenuated virulence in systemic infections [416]. The other two genes -PGA13 and PGA5correspond to mucins that are induced during cell wall regeneration [62]. Another interesting group of down-regulated genes were genes encoding for ribosomal proteins. The expression of 12 genes involved in ribosomal proteins was downregulated in the double mutant cells. It has been reported that the expression of 63 genes out of $117(53 \%)$ is down-regulated during the yeast mode of growth in the gcn5 simple mutant, raising the possibility that Gcn5 is a major regulator of genes involved in ribosomal proteins.

We observed that two (CDR1 and CDR4) of the five putative genes belonging to the multidrug transporter of the ATP-binding cassette $(A B C)$ super family were down-regulated. The over-expression of CDR1 in an azole-resistant strain has been reported [522]. We detected the down-regulation of two multicopper oxidases, FET34 and FET99, required for growth under low iron conditions [424]. The deletion of GCN5 and HDA1 is associated with decreased expression of genes involved in amino acid metabolism and lipid, fatty-acid and isoprenoid metabolism (ERG1, FAS2, SHM2 and SUR2) during the yeast mode of growth. Finally, we detected the down-regulation of EFG1, TYE7 and UCP2. UCP2 is a transcriptional regulator of ergosterol biosynthetic genes and sterol uptake [427]. 


\section{Genome-wide analysis during morphogenesis}

Next, we focused on the identification of genes whose expression was altered in the double mutant strain during the yeast-hypha transition. We generated the transcriptional profiles for the gcn5, hda1 double mutant in Lee medium for 15, 60 and $180 \mathrm{~min}$ at $37^{\circ} \mathrm{C}$ using the CAI4 strain as reference (Figure 3.13).

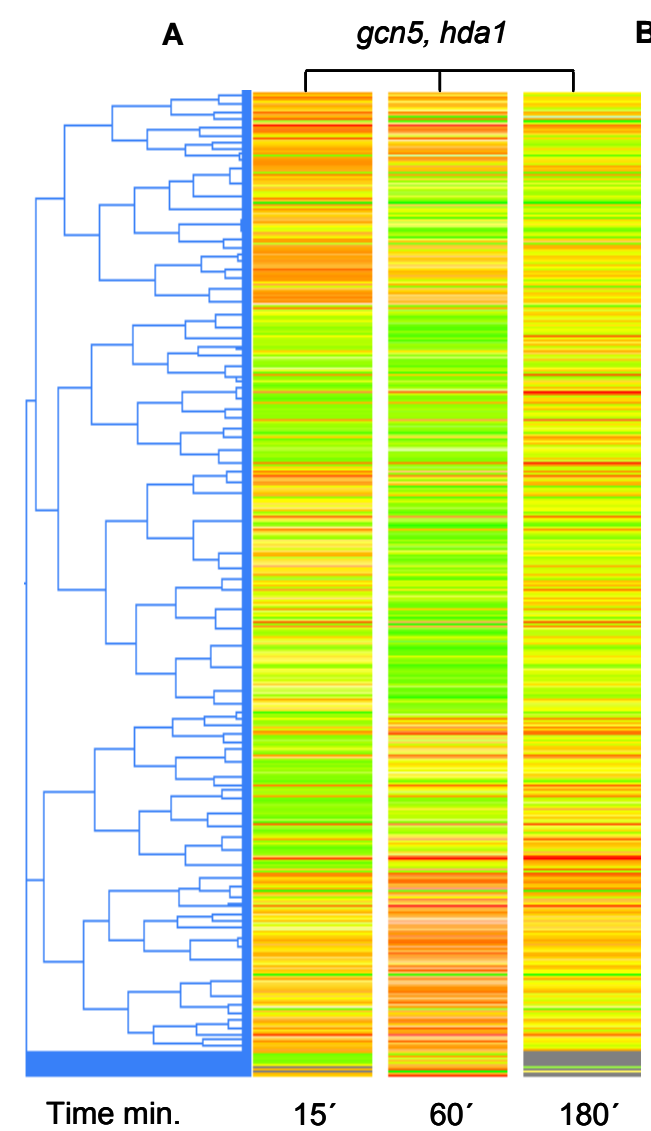

B

Down-regulated genes

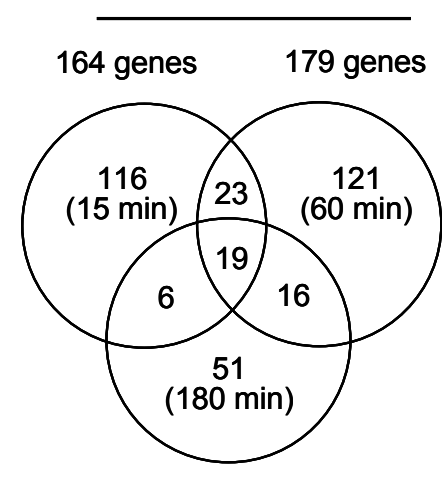

92 genes

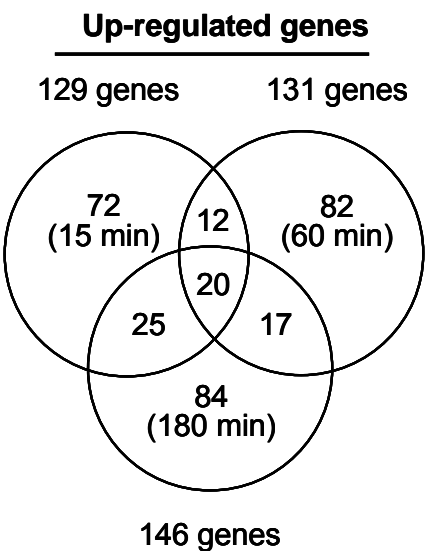

Figure 3.13 Transcript profiles of C. albicans double mutant during the yeast-hypha transition (15, 60 and $180 \mathrm{~min})$ (A). Venn diagrams of genes regulated during different times of the hypha transition. The CAl4 strain was used as a reference for the double mutant ( $g c n 5$, hda1), which was grown in Lee $/ 37^{\circ} \mathrm{C}$ (inducing) (B).

A total of 664 genes were found to change at the consensus level of $\geq 1.5$ fold, including 312 up-regulated and 352 down-regulated genes. The highest foldchange detected was for CA0410, at $60 \mathrm{~min}$, and the lowest fold-change for ECE1, at $180 \mathrm{~min}$. The distribution of genes altered at the three points is represented in the Venn diagram, and the clustering of genes regulated by temperature is represented in Figure 3.13 ( $A$ and $B$ ). 
Table 3.4 Differentially expressed genes during the three times of incubation in the double mutant strain

\begin{tabular}{|c|c|c|c|c|c|}
\hline \multirow{2}{*}{\multicolumn{2}{|c|}{$\begin{array}{l}\text { Gene name Accession no } \\
\text { Up-regulated genes }\end{array}$}} & \multirow[t]{2}{*}{ Description (CandidaDB) } & \multicolumn{3}{|c|}{ Fold regulation } \\
\hline & & & $15 \mathrm{~min}$ & $60 \mathrm{~min}$ & $180 \mathrm{~min}$ \\
\hline \multicolumn{6}{|l|}{ Cell wall } \\
\hline CRH11 & orf19.2706 & putative GPI-anchor; localizes to cell wall & 1.77 & 1.97 & 3.80 \\
\hline KRE1 & orf19.4377 & Predicted GPI-anchor & 1.64 & 1.67 & 3.31 \\
\hline PIR1 & orf19.220 & Putative cell wall protein of the PIR family & 3.85 & 3.28 & 2.55 \\
\hline RHD3 & orf19.5305 & Putative GPI-anchored protein & 2.38 & 1.78 & 7.19 \\
\hline \multicolumn{6}{|c|}{ Oxidoreductase } \\
\hline FRE10 & orf19.1415 & Major cell-surface ferric reductase & 2.37 & 3.46 & 4.18 \\
\hline \multicolumn{6}{|l|}{ Transporter } \\
\hline ENA22 & orf19.6070 & P-type ATPase involved in $\mathrm{Na}^{+}$efflux & 2.63 & 1.90 & 3.66 \\
\hline FTR1 & orf19.7219 & High affinity iron permease & 3.59 & 2.08 & 6.83 \\
\hline GAP5 & orf19.4456 & General amino acid permease & 2.12 & 1.59 & 2.53 \\
\hline HGT10 & orf19.13176 & Sugar transporter & 1.79 & 1.70 & 1.62 \\
\hline PMC1 & orf19.1727 & $\mathrm{Ca}^{2+}$ transporting P-type ATPase & 1.61 & 2.03 & 2.06 \\
\hline SHA3 & orf19.3669 & Involved in glucose transport & 2.85 & 2.00 & 1.71 \\
\hline \multicolumn{6}{|c|}{ Transcription } \\
\hline BDF1 & orf19.978 & Putative ranscription factor & 1.70 & 1.53 & 1.66 \\
\hline GAL4 & orf19.5338 & Transcriptional activator & 1.63 & 1.82 & 2.53 \\
\hline NRG1 & orf19.7150 & Transcriptional repressor Nrg1p/Nrg2p & 1.95 & 1.75 & 2.52 \\
\hline TYE7 & orf19.4941 & Basic helix-loop-helix transcription factor & 2.05 & 1.70 & 2.97 \\
\hline ZCF39 & orf19.7583 & Putative transcription factor & 1.57 & 1.55 & 2.05 \\
\hline \multicolumn{6}{|c|}{ Other function } \\
\hline GAL7 & orf19.3675 & UDP-glucose-hexose-1-phosphate & 2.67 & 1.57 & 2.57 \\
\hline \multicolumn{6}{|c|}{ Unknown function } \\
\hline IPF15217 & orf19.3779 & WD-repeat protein & 1.53 & 1.65 & 1.60 \\
\hline REP1 & orf19.7521 & Unknown function & 2.13 & 1.98 & 3.81 \\
\hline IPF4861 & orf19.6274 & Unknown function & 3.56 & 2.99 & 2.58 \\
\hline \multicolumn{6}{|c|}{ Down-regulated genes } \\
\hline \multicolumn{6}{|l|}{ Cell wall } \\
\hline ALS1 & orf19.5741 & Agglutinin-like protein & 0.40 & 0.52 & 0.45 \\
\hline ALS3 & orf19.2355 & Agglutinin like protein & 0.22 & 0.52 & 0.13 \\
\hline ECE1 & orf19.3374 & Cell Elongation Protein & 0.10 & 0.15 & 0.04 \\
\hline HWP1 & orf19.1321 & Hyphal wall protein & 0.15 & 0.45 & 0.13 \\
\hline MNT1 & orf19.1665 & Mannosyltransferase & 0.32 & 0.45 & 0.53 \\
\hline \multicolumn{6}{|l|}{ Transporter } \\
\hline FCY24 & orf19.7331 & Purine-cytosine transport protein & 0.64 & 0.57 & 0.54 \\
\hline HNM3 & orf19.2587 & Choline permease & 0.53 & 0.37 & 0.52 \\
\hline IPF14040 & orf19.2397 & probable transporter & 0.20 & 0.38 & 0.22 \\
\hline \multicolumn{6}{|c|}{ Vitamin biosynthesis } \\
\hline $\mathrm{BIO} 2$ & orf19.2593 & Biotin synthetase & 0.59 & 0.33 & 0.42 \\
\hline $\mathrm{BIO} 3$ & orf19.2591 & DAPA aminotransferase & 0.60 & 0.50 & 0.47 \\
\hline \multicolumn{6}{|c|}{ Other function } \\
\hline ASC1 & orf19.6906 & A 40 S ribosomal subunit & 0.55 & 040 & 0.55 \\
\hline CYB5 & orf19.7049 & Cytochrome b5 & 0.52 & 0.63 & 0.50 \\
\hline ICL1 & orf19.6844 & Isocitrate lyase & 0.57 & 0.60 & 0.64 \\
\hline FAD2 & orf19.7765 & Delta-12 fatty acid desaturase & 0.60 & 0.34 & 0.57 \\
\hline PFK2 & orf19.6540 & 6-phosphofructokinase & 0.44 & 0.41 & 0.47 \\
\hline POR1 & orf19.1042 & Mitochondrial outer membrane & 0.57 & 0.32 & 0.48 \\
\hline RPL12 & orf19.1635 & Ribosomal protein & 0.59 & 0.56 & 0.45 \\
\hline SUR2 & orf19.5818 & Hydroxylation of C-4 sphingoid & 0.56 & 0.54 & 0.55 \\
\hline \multicolumn{6}{|c|}{ Unknown Function } \\
\hline PET18 & orf19.7330 & Uncharacterized protein & 0.56 & 0.65 & 0.45 \\
\hline
\end{tabular}

Description as Candida Genome date bases 
We found that 39 genes were common to the three times assayed ( 20 and 19 upand down- respectively); 35 genes at 15 and $60 \mathrm{~min}$ (12 up- and 23 down-), and 33 genes at 60 and 180 min (17 up- and 16 down-). 188 genes (72 up- and 116 down), 203 genes (82 up- and 121 down-) and 135 genes (84 up- and 51 down-) varied only at one time of induction (15, 60 and $180 \mathrm{~min}$ respectively). The 39 common genes at the three times are listed in Table 3.4. Only 3 of the common genes corresponded to genes of unknown function. Of the rest of the genes, 13 belonged to disperse functional categories- i.e. CYB5, cytochrome b5; SUR2, hydroxylation of $\mathrm{C}-4$ of the sphingoid moiety of ceramide, etc., and 26 genes could be grouped into four functional categories.

The main category corresponds to cell wall proteins and includes the up-regulation of $R H D 3$, a putative GPI-anchored protein that localizes in the cell wall and whose transcription is decreased upon yeast-hyphal switch [61,104,342], PIR1, a structural protein of the cell wall [309]; KRE1 a predicted GPI-anchor, with a role in 1,6-beta-D-glucan biosynthesis [36,46], and CHR11, a putative GPI-anchor, that localizes to the cell wall $[6,61]$. We observed down-regulation of $E C E 1$, a protein involved in cell elongation [27,32]; of HWP1, a well known cell wall protein [443]; two genes belonging to the ALS family, ALS1 and $A L S 3$, and the alpha-1,2mannosyl transferase, MNT1. All these down-regulated genes have been described previously as being up-regulated during the hypha transition [39,342].

Other genes regulated during all three times of incubation were grouped in the following categories: transporters (9 genes), transcription factors (5 genes) and vitamin biosynthesis (2 genes). As mentioned above, the double mutant strain failed to form hypha under inducing condition and the down-regulation of hyphaspecific genes is consistent with our results.

\section{Expression of transcription factors}

Our microarray analysis indicated that five genes encoding for transcription factors BDF1 [28,302], GAL4 [306], NRG1 [330,331], TYE7 [177,231,281,342], and ZCF39 were up-regulated at three times (Table 3.4). Sixteen genes encoding transcriptional regulators were found to be regulated at one and two times. Twelve of those genes were up-regulated, and four were down-regulated (Supplementary Tables 3.3 and 3.4). Fifteen genes encoding transcriptional regulators (13 up- and 2 down-regulated) have been reported to be regulated by GCN5 at one and two 
times. In the hda1 simple mutant, the up-regulation of TYE7, UME6, ZCF20 and the down-regulation of HAC1 at 15 min of incubation have been reported. Seven of the twenty-one regulated transcriptional factors in the double mutant were overlapped with gcn5 simple mutant. The rest of the transcriptional regulators may be involved in eliciting changes in gene expression that affect the reprogramming of $C$. albicans physiology in response to the deletion of GCN5 and HDA1.

\section{Time-course of gene induction of the main significant categories}

The genes belonging to diverse functional categories whose expression level changed at least at one time of incubation were clustered together. These diverse functional categories include genes that are both up- and down-regulated, indicating a differential regulation of these functions in the double mutant. Several genes can not be readily categorized into any functional group and represent a wide range of cellular metabolic processes (Supplementary Tables 3.3 and 3.4). The microarray results demonstrate that the lack of Gcn5p and Hda1p affects a large number of cell functions and most likely serves to globally reprogram $C$. albicans physiology to allow the double mutant to adapt to this environment.

\section{Cell wall genes:}

Four genes detected by our microarray analysis, ALS1 and ALS3 (at three times), ALS2 (15 $\mathrm{min}$ ) and ALS4 (15 and $60 \mathrm{~min}$ ) encode an agglutinin-like protein and were found to be down-regulated in the double mutant (Figure 3.14). It has been reported down-regulation of $A L S 4$ (15 min), ALS3 (15 and $180 \mathrm{~min}$ ) and ALS1, $A L S 2$ at the three times of incubation in the gcn5 simple mutant.

In the hda1 simple mutant the down-regulation of $A L S 1$ (15 min) and ALS3 (180 min) has been reported. The expression of $A L S$ genes can affect the adhesion [541] of C. albicans, and could be related to the increase in C. albicans virulence under host invasion. This finding and is consistent with the phenotypic results, which indicated that the double mutant failed to form true hypha in either liquid or solid media. Decrease in expression ALS1 and ALS3 (Figure 3.14) in the both simple and double mutant during the yeast-hypha transition suggested that these genes separately regulated by Gcn5p and Hda1p. 


\section{Glucanases, glucosidases and mannosyltransferases:}

Our microarray analysis indicated that twelve genes encoding glucanases, glucosidases and mannosyltransferases were regulated at one, two and three times (Supplementary Tables 3.3, 3.4 and Figure 3.14). Two of these genes were up-regulated (CA5339 and GSL22), and ten were down-regulated (e.g MNT1, GSL21, GLC3, SUN41, MNT3, PMT2 and PMT4). The change in expression of these genes may be involved in eliciting changes in cell wall composition.
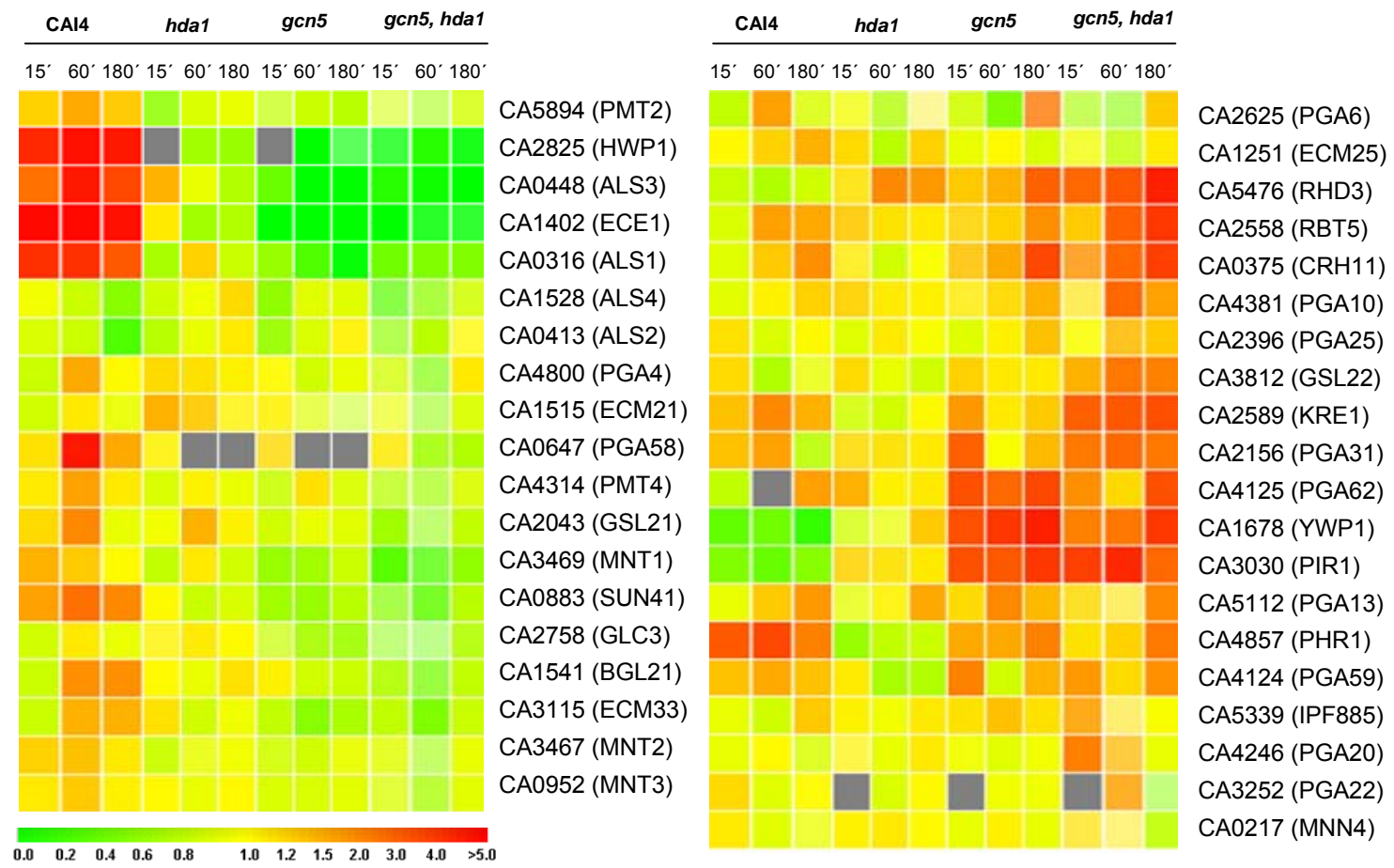

Figure 3.14 Transcriptional regulation of genes involved in the cell wall in the CAI4 strain, both simple and double mutants Representative genes responsible for specific cellular functions are grouped and their regulation during 15, 60 and $180 \mathrm{~min}$ of yeast-hypha transition in the double mutant and strain relative to the CAI4 reference-type strain is listed.

The up-regulation of MNN4 (15 min), BMT4 (180 min) and SUN42 (60 and 180 min) and down-regulation of MNT1 and SUN41 at 15 and 60 min of incubation has also been reported in the gcn5 simple mutant. In the hda1 simple mutant, the down-regulation of PMT1, PMT2 (15 min) and BGL22 (60 min) has been described. These results suggest that, in addition to common regulated genes between both the simple and double mutant cells under the yeast-hypha transition (Figure 3.14), some genes were regulated jointly by GCN5 and HDA1. 


\section{GPI-anchored proteins}

Our results indicate that the transcript levels of sixteen genes belonging to a large class of functionally diverse proteins (GPI-anchored proteins) were regulated in the double mutant at least at one time during the hypha transition. Of these, PGA20 (15 min), PGA22 and PGA25 (60 min), PGA13 and PHR1 (180 min), PGA10 and RBT5 (60 and $180 \mathrm{~min}$ ) and PGA31, PGA59, PGA62 (15 and $180 \mathrm{~min}$ ) were up-regulated and ECM21, ECM25, ECM33, PGA4 and PGA58 (60 min), PGA6 (15 and $60 \mathrm{~min}$ ) were down-regulated (Figure 3.14). GPI-anchored proteins may be enzymes, surface antigens, adhesion molecules, or surface receptors $[86,200,455]$. Ten genes -PGA6, PGA7, RBT1, HYR3, PGA13, PGA10, PGA26, RBT5, PGA62, PHR1 and five genes - PGA48 and PGA62, PHR1 (15 min) and RHD3, PGA59 (60 and $180 \mathrm{~min}$ ) encoding known or putative GPI-anchored proteins have been described to be regulated in the gcn5 and hda1 simple mutant respectively. Our results indicate a role of histone acetyltransferase (GCN5) and histone deacetylase (HDA1), jointly or separately, in the regulation of genes encoding GPI-anchored proteins during the hypha transition.

\section{Genes involved in protein synthesis and ribosomal proteins}

A second important category includes the down-regulation of 19 genes coding for ribosomal proteins and involved in protein synthesis. Twelve of these genes were down-regulated at $15 \mathrm{~min} ; 3$ at $60 \mathrm{~min}, 2$ at $180 \mathrm{~min}$, one at 15 and $180 \mathrm{~min}$ and another one at three times of incubation (Figure 3.15). Only three genes (MRPL36, MRPL17, and RSA2) of this category were found to be up-regulated after 15 and $60 \mathrm{~min}$ in the double mutant strain (Supplementary Table 3.3). No data about the behavior of this group of genes under standard conditions of the yeast-hypha transition (serum or temperature) have been reported, and only 11 are downregulated in the gcn5 simple mutant at one and two times of incubation. Many of them have been described as down-regulated by comparison of the hda1 simple mutant with a CAI4 strain during one and two times of hypha transition (Figure 3.15). 


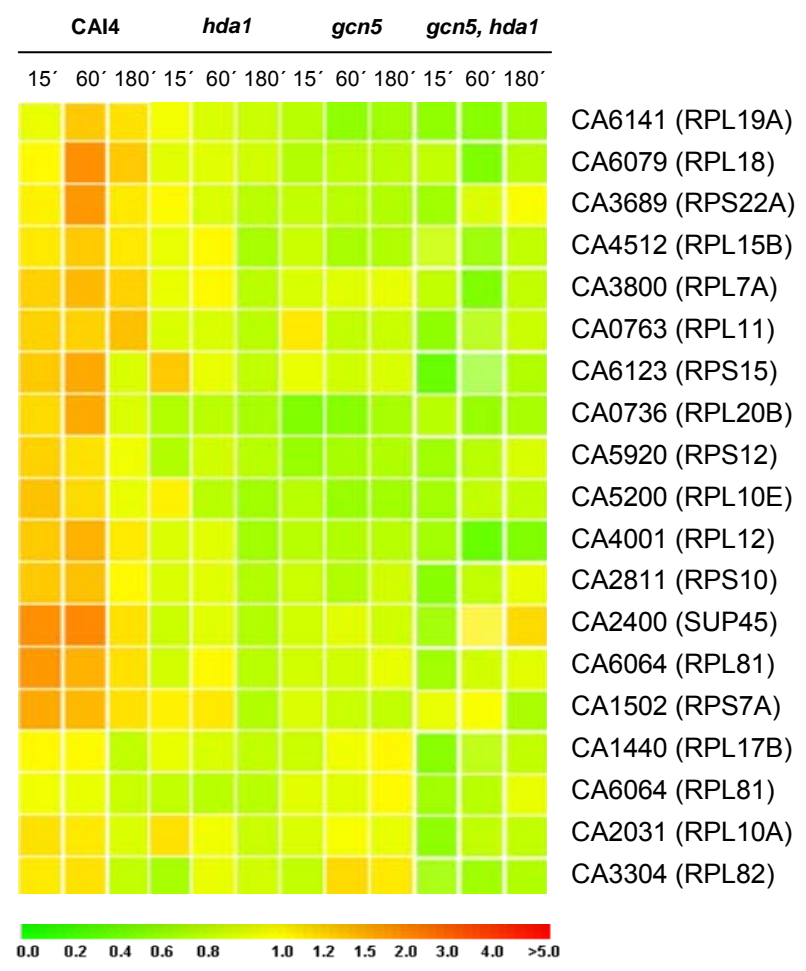

Figure 3.15.Transcriptional regulations of genes coding for ribosomal proteins in the CAI4 strain, both simple and double mutants during the yeast-hypha transiton.

\section{Central carbon metabolism}

The regulation of several genes (18 down-regulated after one, two and three times of incubation) involved in the glycolytic pathway, TCA cycles and gluconeogenesis was detected (Figure 3.16). The expression of most of them increased with time, suggesting an adaptation to the hyphal mode of growth. It has been reported that 20 genes involved in glycolysis and gluconeogenesis are regulated (15 downregulated) in the gcn5 simple mutant and that 20 genes involved in glycolysis, gluconeogenesis and the TCA cycle are up-regulated in the hda1 simple mutant at one, two and three times of incubation (Figure 3.16). The down-regulation of most genes involved in central carbon metabolism in the double mutant and the gcn5 simple mutant is in agreement with dominant of gcn5 phenotype in the double mutant strain. 

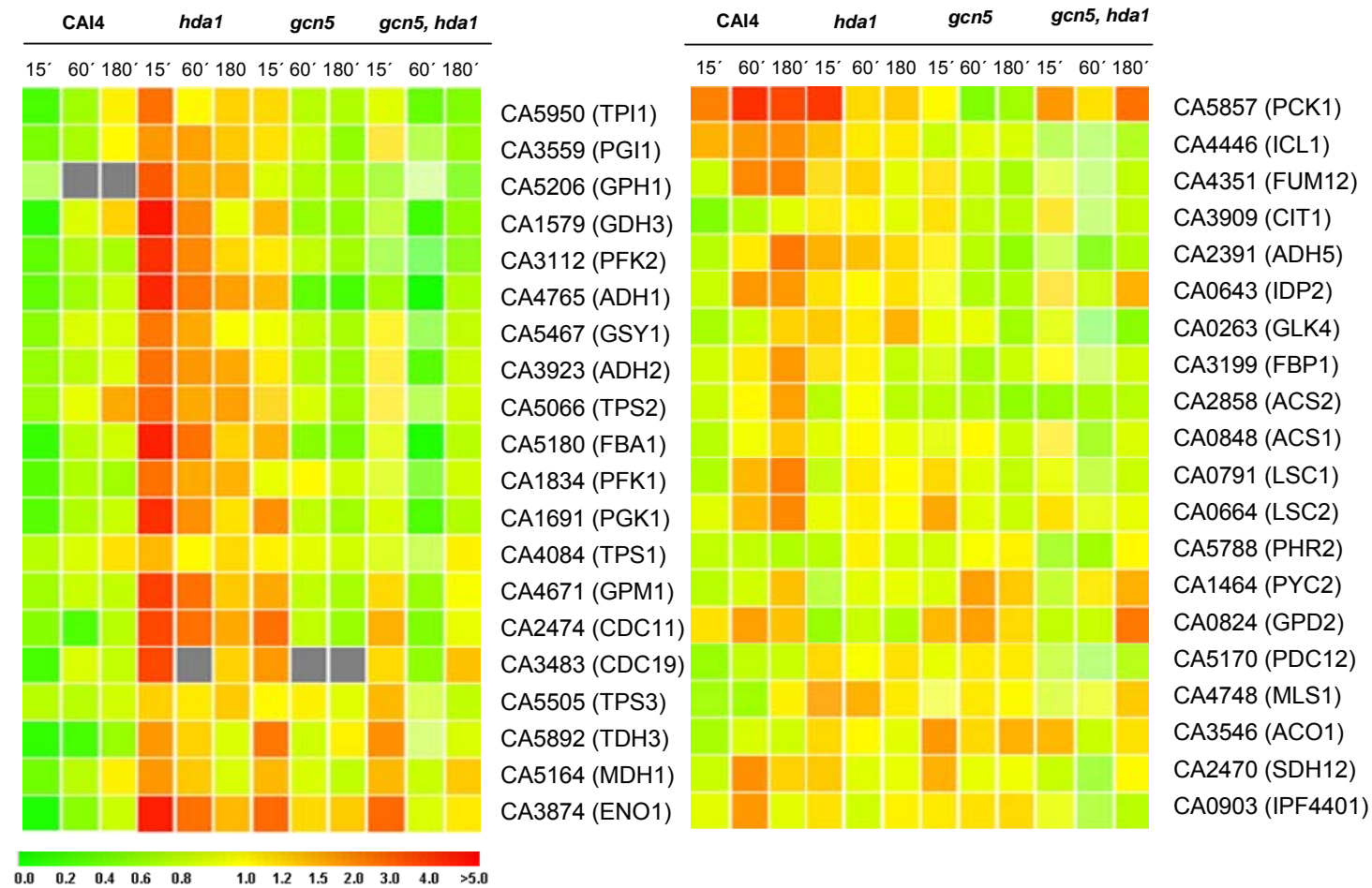

Figure 3.16 Transcriptional regulation of genes involved in the glycolytic pathway in the CAI4 strain, both simple and double mutants during the yeast-hypha transition.

\section{Transporters}

Nine genes were regulated at the three times of incubation (three genes down- and six up-, Table 3.4) and 20 genes at one or two times (Table 3.5). At $15 \mathrm{~min}$, a number of ion transporters including ENA21, PHO84, PMA1 and RHO88 were down-regulated. At $180 \mathrm{~min}$, some genes encoding for amino acid-transporter permeases, including $A G P 1, A G P 3, D I P 51$ and GAP6, were up-regulated. Martin [307] detected up-regulation of genes involved in glucose transport HGT11, HGT12 (15 min), SNF31 (180 min) and HGT7 (15 and $60 \mathrm{~min}$ ) in the hda1 mutant. Degano [106] reported up-regulation of 4 genes encoding for amino acid-transporter permeases at 180 min of incubation in the gcn5 simple mutant. The up-regulation of genes involved in the amino acid-transporter permeases in both the double and gcn5 simple mutant is again in agreement with the dominant of phenotype of gcn5 in the double mutant strain. 
Table 3.5 Regulated genes involved in transport

\begin{tabular}{|c|c|c|c|c|c|}
\hline \multirow{2}{*}{\multicolumn{2}{|c|}{$\begin{array}{l}\text { Gene name Accession no } \\
\text { Up-regulated genes }\end{array}$}} & \multirow[t]{2}{*}{ Description } & \multicolumn{3}{|c|}{ Fold regulation } \\
\hline & & & & & \\
\hline AGP1 & orf19.8784 & Asparagine and glutamine permease & 0.48 & 0.58 & 2.89 \\
\hline AGP3 & orf19.3795 & Amino acid-permease & 1.37 & 0.94 & 1.80 \\
\hline CAN2 & orf19.111 & Amino acid permease & 0.58 & 0.72 & 0.95 \\
\hline CAN5 & orf19.3641 & Amino acid permease & 1.02 & 0.57 & 0.95 \\
\hline CTR1 & orf19.3646 & Copper transport protein & 0.80 & 0.82 & 0.50 \\
\hline DIP51 & orf19.2942 & Dicarboxylic amino acid permease & 1.44 & 1.15 & 1.62 \\
\hline DUR32 & orf19.5017 & Urea transport protein & 0.88 & 0.86 & 0.64 \\
\hline ENA21 & orf19.5170 & P-type ATPase & 0.61 & 1.23 & 1.23 \\
\hline FTR2 & orf19.7231 & High affinity iron permease & 1.34 & 2.15 & 2.57 \\
\hline GAP6 & orf19.6659 & General amino acid permease & 1.12 & 0.97 & 2.20 \\
\hline HGT8 & orf19.2021 & Putative glucose transporter & 1.56 & 0.93 & 2.92 \\
\hline HGT6 & orf19.2020 & Sugar transporter & 1.12 & 0.80 & 1.76 \\
\hline IPF1992 & orf19.7336 & Putative MFS transporter & 1.19 & 1.69 & 1.35 \\
\hline IPF9079 & orf19.4550 & Membrane transporter & 1.23 & 2.27 & 1.21 \\
\hline JEN2 & orf19.12767 & Carboxylic acid transporter protein & 1.85 & 1.81 & 1.43 \\
\hline $\mathrm{PHO} 84$ & orf19.1172 & Inorganic phosphate transport protein & 0.57 & 1.08 & 0.75 \\
\hline PHO88 & orf19.7327 & Involved in phosphate transport & 0.51 & 0.69 & 0.81 \\
\hline PMA1 & orf19.5383 & Plasma membrane $\mathrm{H}^{+}$-transporting ATPase & 0.64 & 0.70 & 0,91 \\
\hline TPO4 & orf19.473 & Putative sperimidine transporter & 0.73 & 0.80 & 0.63 \\
\hline ZRT1 & orf19.3112 & High-affinity zinc transport protein & 0.94 & 1.16 & 0.60 \\
\hline
\end{tabular}

Description as Candida Genome date bases

\section{Vitamin biosynthesis}

We detected the down-regulation of two genes involved in biotin biosynthesis $B I O 2, B I O 3-$ at three times, and BIO4 at 60 min of incubation in the double mutant cells. We identified the down-regulation of two genes involved in thiamine biosynthesis - THI13 (60 min) and THI4- at 15 and $180 \mathrm{~min}$ of induction. The downregulation of $\mathrm{BIO} 2$ at $180 \mathrm{~min}$ and of two genes involved in thiamine biosynthesis (THI4 and THI13) at one and two times of incubation in the gcn5 simple mutant has been described. This result again is in agreement with the dominance of the gcn5 phenotype in the double mutant strain.

\section{Set of genes regulated only at one incubation time}

The up-regulation of two components of the $\mathrm{F}_{1} \mathrm{~F}_{0}$ ATPase complex (ATP2 and ATP16), subunits of cytochrome b and cytochrome c (COB, COX $3 A$ and $C O X 3 B)$, subunits of the NADH ubiquinone oxidoreductase (NAD1, NAD4, NAD5 and NAD6), three genes encoding 26S proteasome (RPN1, RPN9 and RPT4) and two components of superoxide dismutase (SOD1 and SOD2) were detected at $15 \mathrm{~min}$ of incubation. Of those genes grouped in the secretory pathway (ERV25, ERV46, SEC24 and SEC31) and methionine biosynthesis (CYS3, MET2, MET3, MET10, MET14, MET15 and SAM2) were down-regulated at $15 \mathrm{~min}$ of induction (Figure 3.17). Some of these genes (MET3 and MET14) overlap with the gcn5 simple 
mutant at 15 min of incubation. No genes of this category were found up-regulated or down-regulated at any time in the hda1 simple mutant.

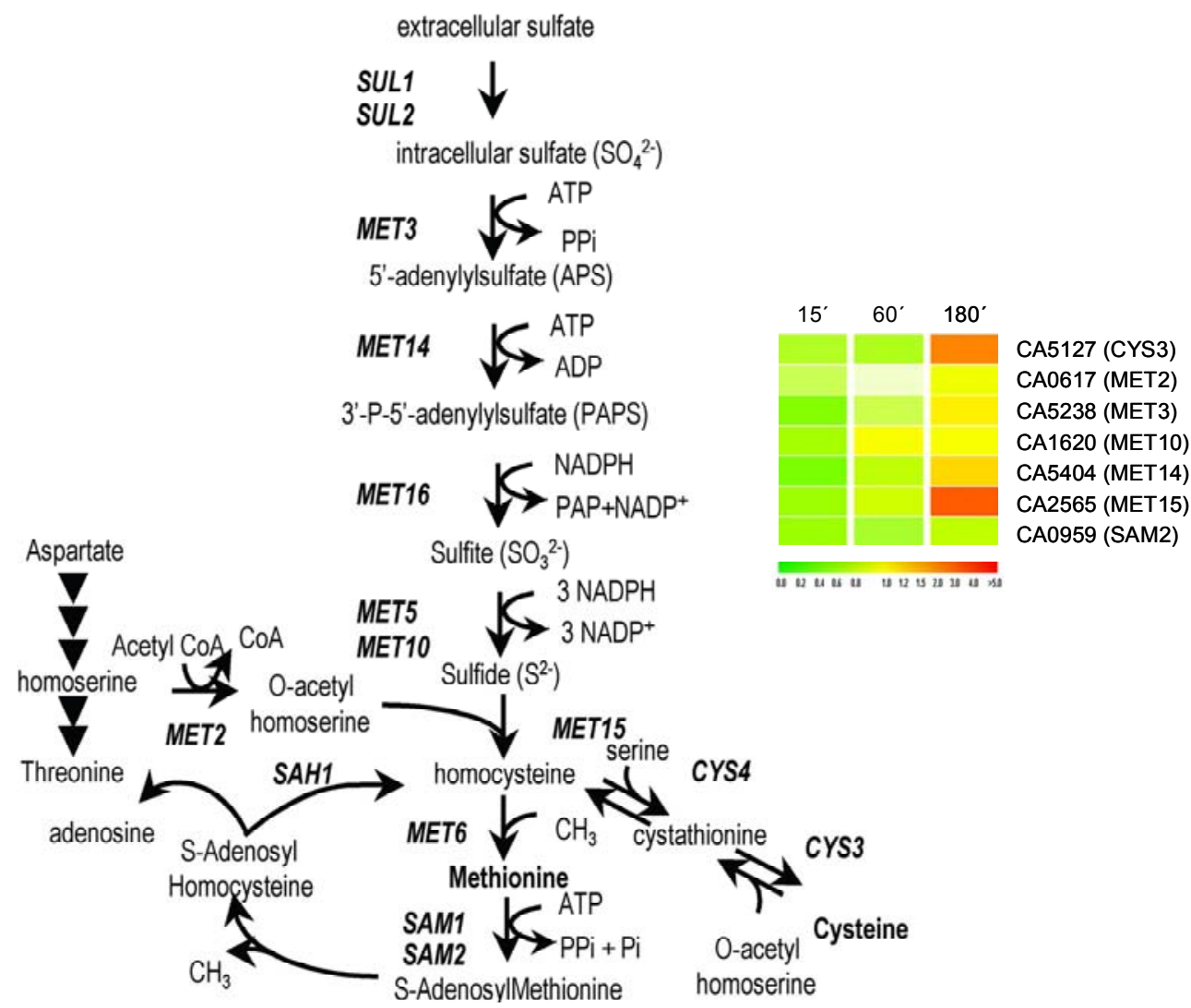

Figure 3.17 Differential expressions of the genes involved in sulphur amino acid biosynthesis in the gcn5, hda1 double mutant strain.

At $60 \mathrm{~min}$ of incubation, the down-regulation of genes involved in ergoestrol biosynthesis (Figure 3.18) and respiration (COX12, CYT12, NUC2 and QCR8) was observed. At 60 and $180 \mathrm{~min}$, we detected regulation two and four genes encoding histones HHF1, HHF22, HHT1, HHT21 and HTB1. However three of these genes, HHF21, HHF22 and HHT1, were up-regulated in the hda1 simple mutant [307]. While five genes were down-regulated in the gcn5 simple mutant [106].

These results suggest a remodelling of chromatin, probably for it to be adapted to a new transcriptional profile. No specific set of down-regulated genes was found at $180 \mathrm{~min}$ of incubation, suggesting that at this time the double mutant cells had reached a general balance in their main regulatory metabolic pathways. 


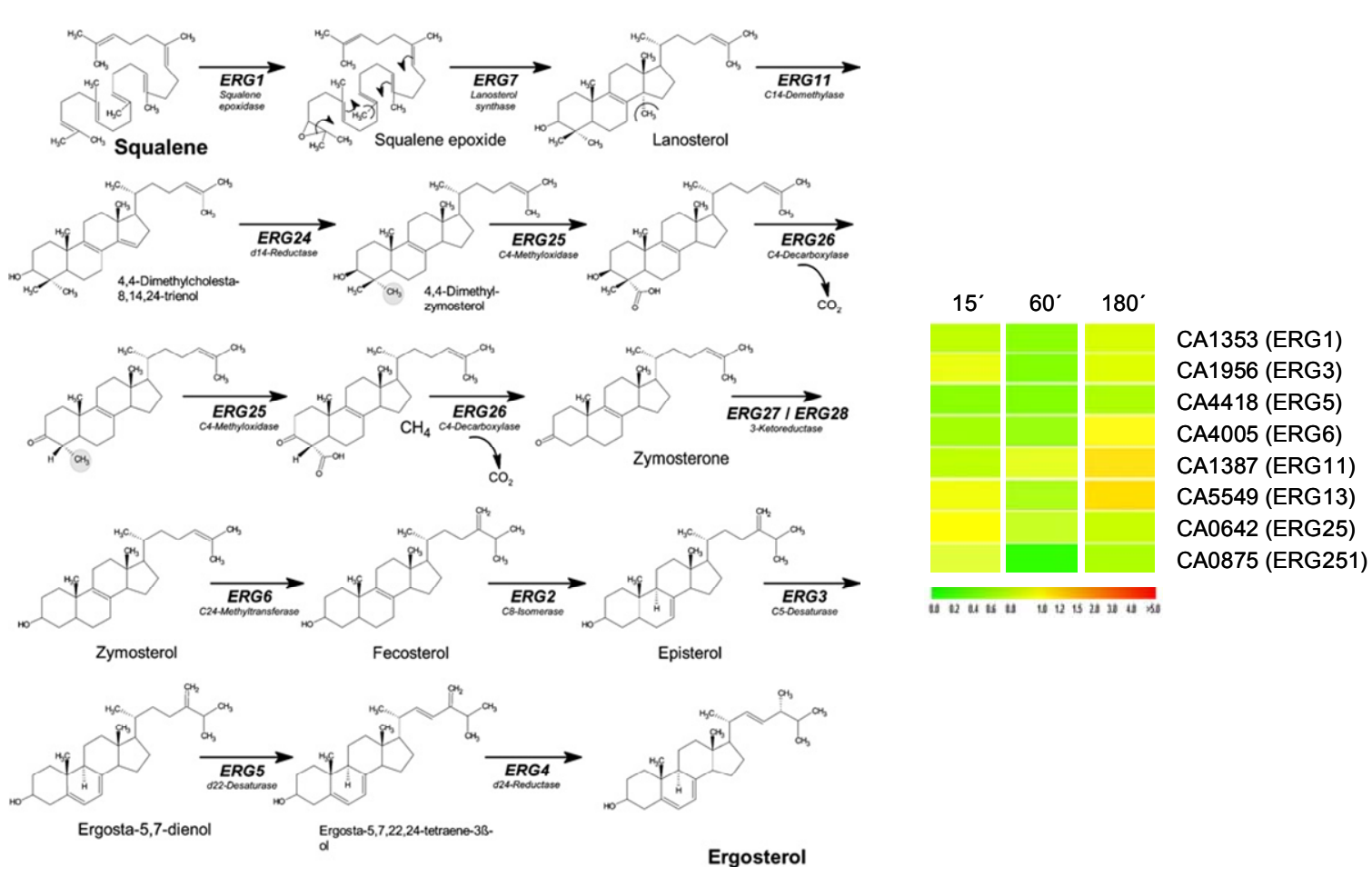

Figure 3.18 Sphingolipid biosynthesis pathway in the yeast $\mathbf{S}$. cerevisiae according to [486]. Differential expression of the genes involved in sphingolipid biosynthesis. Expression ratios of the four paired comparisons (log scale) are represented during the yeast-hypha transition (15, 60 and $180 \mathrm{~min})$.

\section{Discussion}

Candida albicans is able to establish mucosal and invasive disease by means of different virulence factors of unknown regulation. The focus of our work was on understanding the possible compensatory effects of deletion in the same strain of a histone acetyltransferase (GCN5) and histone deacetylase (HDA1) by analyzing the whole transcriptional profile during the yeast mode of growth and during the yeast-hypha transition. It has been reported that HDAC inhibitors cause a $90 \%$ reduction in the adherence of $C$. albicans to human cultured pneumocytes and significantly inhibit serum-induced germination [426]. The functions of GCN5 and HDA1 on the known filamentation pathways in C. albicans are as yet unknown. In our laboratory the GCN5 and HDA1 genes, have been previosly deleted in a CAI4 strain. In this work, we deleted the GCN5 gene in an hda1 mutant background strain. Deletions of GCN5 appeared to block hyphal formation under a variety of standard inducing conditions; for example in the presence of serum or GlcNAc or during growth on Spider medium [106]. The deletion of HDA1 does not block hypha formation in all liquid media, but does block the formation of true hypha in some solid media [307]. In the present work we show that deletion of the GCN5 and 
HDA1 such as the gcn5 simple mutant strain is associated with a detectable deficiency in the rate of colony formation during growth on rich-medium plates at $28^{\circ} \mathrm{C}$. Furthermore, there was a significant difference between the growth rate of the double mutant and CAI4 during exponential growth in rich liquid medium at $28^{\circ} \mathrm{C}$. Morphologically, like the gcn5 simple mutant, the gcn5, hda1 double mutant is very different from the CAI4 strain. The main phenotype of the double mutant is its inability to switch from the yeast to the filamentous form in liquid media containing serum or at $37^{\circ} \mathrm{C}$ and in all the solid media assayed. Our data suggest that the lack of Gcn5p down-regulates alternative morphogenetic pathways, thereby preventing filamentation under these conditions. However, Gcn5p is required for maintenance of the yeast growth form because the gcn 5 mutant growth like small pseudohypha. Our results show that in the gcn5, hda1 double mutant, as well as in the gcn5 simple mutant, chlamydospore formation is completely abolished. Chlamydospores can be induced to form under nutrient-poor oxygenlimited conditions at low temperatures [52]. Chlamydospores have been found in the lung of AIDS patient $[80,353]$, and thus may be relevant to infection. The ability to form chlamydospores is almost universal among the $C$. albicans clinical isolates [2], thus suggesting that chlamydospores would have a functional role in $C$. albicans biology. A recent study has shown that in C. albicans genes such CYP56, ISW2, MDS3, RIM13, RIM101, SCH9 and SUV3 are required for efficient chlamydospore formation [317,353], we have found that GCN5 is another gene required for efficient chlamydospore formation.

In the double mutant cells, a total of 664 genes, out of 6039 present in the microarray, changed due to deletion of GCN5 and HDA1, in a minimal medium (Lee) at one of the three incubation times assayed (15, 60 and $180 \mathrm{~min}$ ). These represent a percentage of $10.99 \%$. 180 genes, while only $2.98 \%$ of the genes changed during the yeast form of growth. Only five transcription factors were upregulated at the three times assayed and others were regulated at two times (five), or at one time (nine). The regulation of some well characterized transcription factors e.g., NRG1 [330] and UME6 [22] is in agreement with the phenotype of the double mutant cells during hypha development.

We found, regulation at least 40 genes corresponding to well cell wall proteins. Four of the repressed genes, ALS1 [205], ALS2 [204], ALS3 [203] and ALS4 [204] are all classified in the same subfamily [200]. We detected the down-regulation of 
ALS2 and ALS4 during the yeast form growth in both the simple and double mutants. The down-regulation of this group of genes is in agreement with the significant increase in the binding of the positively charged dye Alcian blue, suggesting an increase in cell wall phosphate contents in the double mutant cells. The down-regulation of $A L S 2$ and $A L S 4$ during the yeast form of growth and the increase in the binding of the positively charged dye Alcian blue has been reported in both simple mutants. We also observed the down-regulation of PGA4, PGA6, PGA58 and the up-regulation of PGA10, PGA13, PGA20, PGA22, PGA25, PGA31, $P G A 59$, and $P G A 62$ at one and two times of incubation, suggesting a cell wall remodelling during the hypha transition. We detected the down-regulation of SUN41 $(0.60,0.49,0.78)$ during the hypha transition. The inactivation of SUN41 affords defects in cell separation and hyphal elongation [130] and elicits a higher sensitivity towards the cell wall-disturbing agent Congo red [191]. In agreement with the down-regulation of SUN41, our mutant also showed higher sensitivity towards the cell wall-disturbing agent Congo red. Another group of down-regulated genes included ECM21, ECM25 and ECM33 and in C. albicans, these are involved in cell wall biogenesis and architecture [281]. ECM33 is a GPI-anchored protein and is important for proper cell wall ultrastructural organization and also for the correct assembly of the mannoprotein outer layer of the cell wall. We found that the double mutant was sensitive to CWF, $\mathrm{NaCl}$, Caffeine and SDS. This result confirms that the double mutant cells undergo a change in cell wall composition. We observed the down-regulation a number of genes involved in lipid, fatty acid, and sterol metabolism, such as CYB5, ERG1, ERG3, FAS1 and FAS2 [281]. The decrease in the expression of a number of genes involved in lipid, fatty acid, and sterol metabolism is in agreement with the sensitive phenotype of the double mutant to Fluconazole.

The expression of genes involved in glycolytic pathway and carbohydrate metabolism was decreased in the double mutant in both the yeast and hyphal forms. The same genes were not always identified during yeast mode of growth and during the yeast-hypha transition (Table 3.3 and Supplementary Table 3.4), suggesting a specific regulation that deserves further investigation. It has been demonstrated that the addition of glycolytic inhibitors blocks filamentation in $C$. albicans in germ-tube-inducing medium [260], indicating that the glycolytic pathway is required for filamentous growth. The genes involved in glycolysis and in reserve 
carbohydrates were down-regulated in the yeast mode of growth and during the yeast hypha transition, as in the APSES mutants [108]. A large number of genes involved in glycolysis and gluconeogenesis has been reported to be downregulated in the gcn5 deletion strain, especially at one and two times of incubation, and to be up-regulated in the hda1 simple mutant, suggesting that a common pathway may be regulated differently in both single mutants. Our results demonstrated that the double mutant strain underwent a metabolic change, including genes involved in carbohydrate metabolism during the yeast mode of growth and the yeast-hypha transition. It has been reported that genes involved in carbohydrate metabolisms do not appear as an important group in response to the treatment of $C$. albicans with antifungal agents [281]. Barrelle et al [23] have shown that the progression of systemic disease caused by $C$. albicans is dependent on glycolysis. Strains that are deleted for some glycolysis specific genes have a dramatically reduced virulence in animal models [23]. In addition, it has been reported that PFK2 (also specific for glycolysis) is highly expressed during systemic infection. It is therefore possible that the dramatic reduction in virulence seen in the gcn5 deletion mutant could be caused, at least partially, by a down-regulation of glycolysis.

We observed the down-regulation of components of the translation apparatus: 19 genes during one, two and three times of incubation. The down-regulation of this group of genes has been reported in previous work describing the yeast-hypha transition of both the gcn5 and hda1 simple mutant strains. We described an upregulation of components of the translation apparatus in yeast-hypha transition of the CAI4 strain due to a change of temperature in chapter II. We also detected the down-regulation of most of these genes during the yeast mode of growth. In another work carried out at our laboratory, it has been reported that in the gcn5 simple mutant 63 out of 117 down-regulated genes during the yeast mode of growth coded for components of the translation apparatus. These results suggested that Gcn5p would mainly be involved in the regulation of the components of the translation apparatus.

Finally, many genes related to transport (Table 3.5), methionine synthesis and vitamin synthesis were regulated in the double mutant strain. Several of them are related to iron transport or encode general amino acid permeases. No previous data have been reported for the regulation of these groups of genes during the 
hypha transition.

Overall, the transcript levels of a significant number of genes were differentially expressed in the gcn5 or the hda1 strains, suggesting that there are several pathways that may be regulated in a different way by both proteins. 


\section{Global transcriptome of Candida albicans odc null mutant during the} yeast mode of growth and during the yeast-hypha transition

\section{Abstract}

By comparison of the transcriptional profiles of a $C$. albicans odc/odc mutant during the yeast mode of growth in the exponential phase and during the yeast-hypha transition at low $(0.01 \mathrm{mM})$ and high $(10 \mathrm{mM})$ polyamine levels, we found that about one-third $(31.14 \%)$ of the genes were differentially regulated. A total of 137 and 1981 genes changed during the yeast mode of growth and during the yeast-hypha transition, respectively. $502(25.39 \%)$ genes commonly change at the three times of the yeast-hypha transition.

The main categories of up-regulated genes during the yeast mode of growth were cell wall, histone assembly and transport. The main down-regulated genes corresponded to those coding for cell wall proteins, drug resistance, glycolytic pathway, glucose transport and transcription factors.

The genes differentially expressed at the three times of yeast hypha transition are (i) genes coding for amino acid permeases (AGP1, CAN2, CAN5, HNM3, IPF4580 and MAL31), (ii) genes coding for (a) cell-wall formation (downregulated: AAF1, ALS3, BGL22, CHS5, DPM1, ECE1, ECM17, ECM33, FGR6, GLC3, HYR1, HWP1, IPP1, PGA26, PGA36, PGA48, PGA53, PGA54, PGA59, RBT1, SUN41; and up-regulated: BMS1, ECM1, MNN11, MNN4, PGA31, PHR2, PIR1), cell polarity and filament formation (ACT1, ARF3, ARP3, BET3, CDC12, CDC23, CHC1, COF1, GDI1, IPF1022, MHP1, RDI1, SEC24, TEM1 and TPM2); (b) ergosterol biosynthesis (DAP1, ERG1, ERG24 and ERG251); (c) glycolysis (CDC19, ENO1, FAB1, FBA1, GPM1, HXK2, PDC11, PDI1, PFK1, PFK2 and PGK1); (d) lysine biosynthesis (LYS1, LYS12, LYS7 and LYS9); (e) respiration and electron transport (ATP1, COX13, COX3A, COX3B, COX4, CUP5, IPF6566, NAD6, STF2, VMA1 and VAM6), (iii) transcription factors (down-regulated: GCF1, IPF29, IPF9826, TIP120, TYE7 and ZCF4; and up-regulated: ACE2, BCR1, BRE1, CAP1, CTA8, DAL81, GCN4, INO2, IPF6857, NRG1, RMS1, SFU1, TAF19, TBP1, WOR2, ZCF3 and ZPR1), (iv) DNA-directed RNA polymerases (RPB5, RPB8, RPC25, RPC40, RPC53, RPC82 and PAF1), (v) mitochondrial ribosomal proteins (IPF3361, IPF16564, MRP1, MRP17, MRP20, MRP7, MRPL17, MRPL40, MRPS28 and YML6 ), (vi) nucleolar proteins (ENP1, ENP2, NOP1, NOP14, NOP15, NOP2, NUP133 and NUP84), (vii) cytoplasmic ribosomal proteins (RPF1, RPL23, RPN4, 
RPS27A, RRP42, RRP6, RRP8, RRP9, RRS1 and IMP3), (viii) RNA helicases (DBP2, DBP3, DBP8, DBP9, CHR1, DRS1, HAS1, PF15646, MTR4, RRP3 and SUV3) and (ix) translation initiation factors (CDC95, GCD11, GCD7, NIP1, SUA5, SUI1, SUI3 and TIF1). About $32 \%$ of the differentially regulated genes included transcripts encoding products not yet classified for their functional attributes. The results suggest that polyamine levels exert a pleiotrophic effect on transcriptional activity.

\section{Introduction}

Candida albicans is an opportunistic fungus that generally exists in the oral cavity, skin, vagina and intestinal organs [340]. C. albicans is dimorphic and undergoes transition from the yeast form to the hyphal form depending on the growth conditions [155,451]. It grows in yeast form on membranes and skin surfaces, but in the hyphal form, it occurs at deep-seated mycoses [151]. The hyphal transition is induced by activation of the MAP kinase and cAMP pathways, which are controlled by the RAS protein $[16,17,218,403]$. Polyamines are synthesised from ornithine by ornithine decarboxylase (ODC) and are inducers of the enzyme adenylate cyclase $[285,476]$. Polyamines are essential for cell growth, and their metabolism is extensively regulated [507]. The $C$. albicans ODC gene (ODC1), showing high homology with other eukaryotic ODCs at both the amino acid and nucleic acid levels, is required for the synthesis of polyamines [188,316,350]. ODC1 catalyses the conversion of ornithine into putrescine, which is the first step in the universal mechanism of polyamine biosynthesis, and it is one of the most highly regulated enzymes of eukaryotic cells $[69,101,206,219]$. This control is essential, because excess polyamine is toxic and induces apoptosis [361,371]. The most important control mechanism of $O D C 1$ in eukaryotic organisms operates at the posttranslational level, regulating the amounts of the enzyme by a unique mechanism of proteasome-dependent degradation $[180,186]$. Because of the narrow concentration range of polyamines needed for execution of their multiple roles, ODC1 expression is tightly controlled. Even modest reductions in mammalian ODC1 activity can lead to marked resistance to tumour development $[348,365]$. Intracellular levels of polyamines are regulated at various steps, including synthesis, degradation, uptake and excretion, and cells have developed intricate mechanisms to ensure a tight regulation of intracellular polyamine pools $[68,492]$. 
In fungi, polyamines are essential for distinct differentiation processes, such as spore germination, sporulation and dimorphism [395]. The possible significance of the transcriptional regulation of the gene encoding $O D C 1$ by polyamines in fungi may depend on its interrelation with the post-translational regulation of the enzyme. The simultaneous operation of transcriptional and post-translational regulatory mechanisms has been reported to endow the cell with a more efficient mechanism to control the physiological processes that depend on polyamines [220]. To study polyamine regulation in $C$. albicans, a odc/odc mutant, which behaved as a polyamine auxotroph and grew exclusively in the yeast form at low polyamine levels (0.01 mM putrescine) under all conditions tested, has been generated [188]. An increase in the polyamine concentration (to $10 \mathrm{mM}$ putrescine) restored the capacity to switch from the yeast form to the filamentous form, proving that polyamine concentrations are essential for the switchover [188]. Deletion of $C$. albicans ODC gene provide an excellent genetic system to study the effects of polyamines on gene expression during the yeast mode of growth and during the yeast-hypha transition, thereby facilitating the exploration of the possible mode of their regulation.

In this work, we report a study of the transcriptional profile of a C. albicans odc/odc mutant during the exponential phase and during the yeast-hypha transition at low $(0.01 \mathrm{mM})$ and high $(10 \mathrm{mM})$ levels of putrescine.

The information thus generated has enabled us to develop our knowledge about polyamine-mediated coordination between different levels of regulation, which may eventually explain the role of polyamines in yeast-form growth and hyphal transition in C. albicans.

\section{Materials and methods}

\section{Yeast strain, media and growth conditions}

The strain of $C$. albicans used was ABH4 (ura3/ura3 odc1::hisG/odc1::hisG) [188]. The strain was grown in a medium containing $1.25 \%$ glucose as a carbon source and supplemented with $0.2 \mathrm{mM}$ uridine. Solid medium was obtained by adding agar (2\%). The dimorphic transition was induced by changing the temperature to $37^{\circ} \mathrm{C}$. Putrescine was added at low and high concentrations $(0.01 \mathrm{mM}$ and $10 \mathrm{mM}$, respectively). 


\section{RNA isolation, microarray design and preparation}

For yeast-form growth of the $C$. albicans odc mutant strain, $\mathrm{ABH} 4$ was grown in Lee medium cultures supplemented with $0.01 \mathrm{mM}$ and $10 \mathrm{mM}$ of putrescine till the midlog phase $\left(O D_{600 n m}, 1.5\right)$. The dimorphic transition was induced by changing the temperature to $37^{\circ} \mathrm{C}$. Cell were pre-grown at $28^{\circ} \mathrm{C}$ in Lee medium without polyamine until the exponential phase, washed, starved in water for $24 \mathrm{~h}$ at $4^{\circ} \mathrm{C}$ and incubated in Lee medium $\left(\mathrm{pH} 6.8,37^{\circ} \mathrm{C}\right)$ supplemented with either $0.01 \mathrm{mM}$ or $10 \mathrm{mM}$ of putrescine. Cells were harvested at room temperature, resuspended in a very small volume of the supernatant and immediately frozen by releasing small drops of cell samples into liquid nitrogen. Cells were then stored at $-80^{\circ} \mathrm{C}$ until RNA extraction was carried out. Total RNA isolation, microarray design and cDNA microarray data analysis were carried out as described in Chapter II.

\section{Results}

\section{Global transcriptomal response of the odc mutant}

The C. albicans odc/odc mutant grown at low polyamine levels $(0.01 \mathrm{mM}$ of putrescine) failed to form filaments in response to media with serum or any other known inducers of filamentous growth. In addition, null strain was hypersensitive to Calcofluor and salts, indicating that polyamine levels control a broad range of gene functions [188]. A comparison of the transcript profiles between the low $(0.01 \mathrm{mM}$ putrescine) and high (10 mM putrescine) polyamine levels was first ascertained using cDNA microarrays. The data analysis was based on statistical confidence ( $p$ $<0.02$ ) and at least 1.5-fold expression change. Initially, a comparison of odc mutants growing in Lee medium at $28^{\circ} \mathrm{C}$ with low $(0.01 \mathrm{mM})$ and high $(10 \mathrm{mM})$ levels of putrescine in the exponential growth phase $\left(O D_{600 n m}, 1.5\right)$ was carried out. The deletion of $O D C$ produced $\geq 1$.5-fold changes in the expression of 137 genes, with 52 up-regulated and 85 down-regulated genes during the yeast mode of growth. When transcriptomes of cells growing in Lee medium at $37^{\circ} \mathrm{C}$ during yeasthyphal transition (15, 60 and $180 \mathrm{~min}$ ) were compared, 1981 genes had modified expression levels: 1056 genes were up-regulated and 925 were down-regulated. As we have found in the other $C$. albicans mutants the number of misregulated genes in the mutant was greater during the yeast hypha transition than during the yeast-mode of growth (Figure 4.1). 


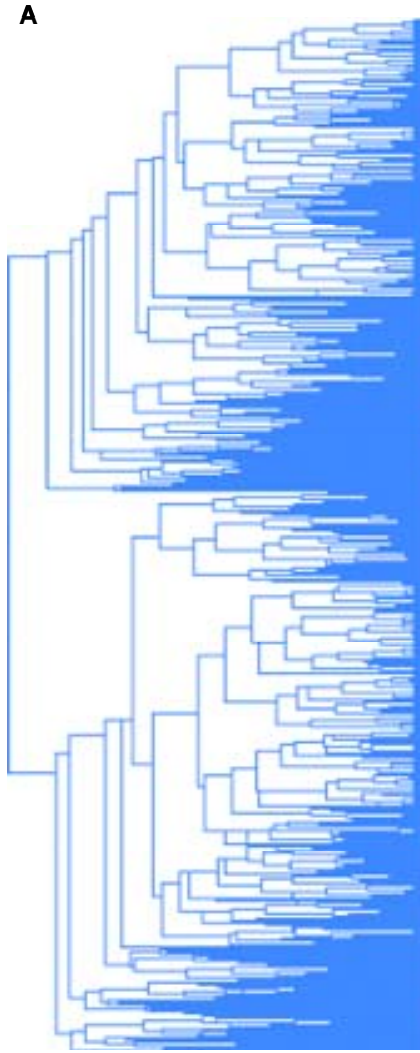

Time min.

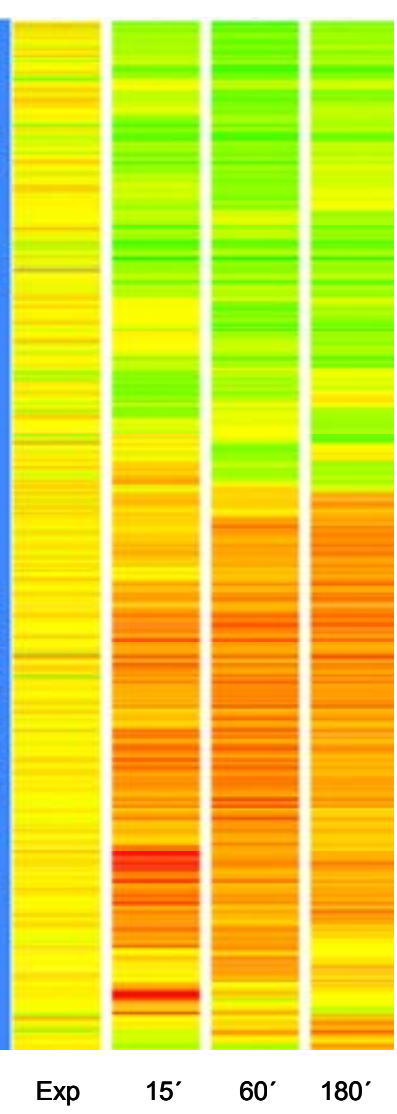

B
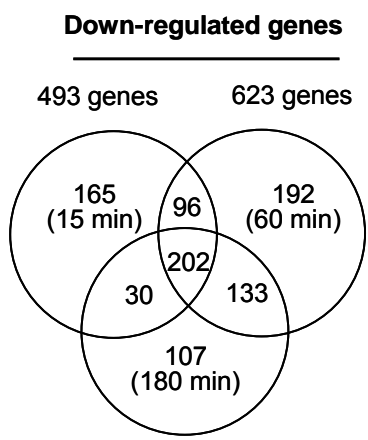

472 genes

Total 925 genes

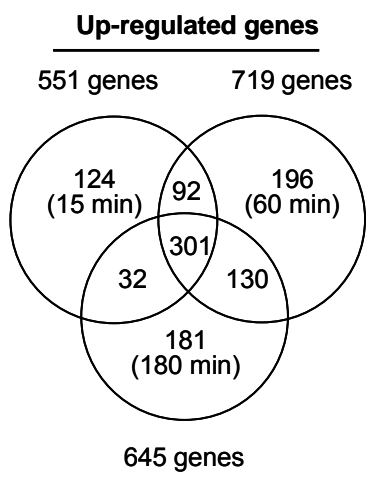

Total 1056 genes

Figure 4.1 (A) Transcriptional Genes Tree and statistical comparisons of polyamines-responsive genes in a C. albicans odc/odc mutant strain grown in Lee medium supplemented with $0.01 \mathrm{mM}$ of putrescine vs $10 \mathrm{mM}$ of putrescine. The transcriptional profiles of genes whose transcript levels responded significantly $(\rho<0.02)$ shift to the $0.01 \mathrm{mM}$ of putrescine (odc mutant strain) were clustered using a gene tree program of Genespring Gx 7.1.1). Panel Exp is a Gene Tree map of the transcriptional changes in gene expression in the yeast mode of growth shift to $0.01 \mathrm{mM}$ of putrescine relative to the $10 \mathrm{mM}$ of putrescine (control cell). Green indicates down-regulated expression and red indicates up-regulated expression. Panels 15, 60 and 180 min show the response of the same genes shown in panel EXP but during the yeast-hypha transition $(15,60$ and $180 \mathrm{~min})$. C. albicans Gene Tree transcriptional pattern indicate genes that showed a differential response $(\rho<0.02)$ during the yeast-hypha transition in the Lee medium $(0.01 \mathrm{mM}$ of putrescine versus $10 \mathrm{mM}$ of putrescine) (B) Venn diagram for genes whose expression was significantly altered during the yeast-hypha transition.

\section{Transcriptome of odc mutant strain in exponentially growing cells}

The analysis of the microarray data of cells in the exponential growth phase showed 137 affected genes (Supplementary Tables 4.1 and 4.2). Of the total number of altered genes, $38(27.73 \%)$ were genes with unknown functions. Among the rest of the genes, RBT5, encoding a glycosylphosphatidylinositol (GPI) cell-wall protein that is regulated by $\operatorname{Rfg} 1 \mathrm{p}, \operatorname{Rim} 101 \mathrm{p}$, Tbf1p, Sfu1p, Hog $1 p$ and Tup $1 \mathrm{p}$ $[29,37,196,227,259,441]$, showed the highest change (3.51 fold), whereas the lowest expression level in this experiment was observed for $H G T 7$, which encodes a putative protein involved in glucose transport, ( -6.17 fold) [124]. Genes expressed differentially between the low $(0.01 \mathrm{mM})$ and the high $(10 \mathrm{mM})$ levels of putrescine are listed in Supplementary Tables 4.1 and 4.2. Endogenous increase in polyamines has been reported to result in significant changes in gene-expression 
patterns [442]. Due to the large number of genes found to be up- or downregulated, this analysis was focussed on main the functional categories detected (e.g cell wall formation, drug resistance, hexose transporters and histone assembly) (Table 4.1).

Table 4.1 Distribution of differentially expressed genes during the yeast mode of growth into functional categories genes that are at least 1.5-fold up- or down-regulated in the $C$. albicans odc/odc. Growth was carried out in Lee medium supplemented with $0.01 \mathrm{mM}$ and $10 \mathrm{mM}$ of puterescine.

\begin{tabular}{|c|c|c|c|}
\hline Gene name & Accession no & Description (CandidaDB) & Fold regulation \\
\hline
\end{tabular}

UP-regulated genes

\begin{tabular}{ll} 
Cell wall & \\
PGA10 & orf19.5674 \\
PGA45 & orf19.245 \\
PGA7 & orf19.5635 \\
PRA1 & orf19.311 \\
RBT5 & orf19.5636 \\
\multicolumn{2}{l}{} \\
Histone assembly \\
HHF21 & orf19.1059 \\
HHF22 & orf19.185 \\
HTA1 & orf19.692 \\
HTB1 & orf19.6925
\end{tabular}

Transcription factor

GCN4

orf19.1358

Transporter

FRE5

HGT14

IPF1992

JEN1

orf19.5634

orf19.2633

orf19.7336

orf19.7447

\section{Other Function}

$\begin{array}{ll}\text { ACH1 } & \text { orf19.10681 } \\ \text { CDC54 } & \text { orf19.11245 } \\ \text { CSA1 } & \text { orf19.7114 } \\ \text { CSA2 } & \text { orf19.3117 } \\ \text { FEN11 } & \text { orf19.6343 } \\ \text { HMO1 } & \text { orf19.6645 } \\ \text { IFQ4 } & \text { orf19.1464 } \\ \text { INO1 } & \text { orf19.7585 } \\ \text { IPF4164 } & \text { orf19.5455 } \\ \text { LSP1 } & \text { orf19.3149 } \\ \text { MRS4 } & \text { orf19.2178 } \\ \text { NHP6A } & \text { orf19.4623.3 } \\ \text { OPS4 } & \text { orf19.4934 } \\ \text { SPB8 } & \text { orf19.7290 } \\ \text { UBI4 } & \text { orf19.6771 }\end{array}$

Unknown function

23 genes

Down-regulated genes

Amino acid permease

$\begin{array}{ll}\text { AGP1 } & \text { orf19.8784 } \\ \text { GAP5 } & \text { orf19.4456 } \\ \text { Cell wall } & \\ \text { ALS1 } & \text { orf19.5741 } \\ \text { BMT4 } & \text { orf19.13055 } \\ \text { HYR1 } & \text { orf19.12440 } \\ \text { IPF3964 } & \text { orf19.675 } \\ \text { PGA26 } & \text { orf19.2475 } \\ \text { PGA31 } & \text { orf19.5302 }\end{array}$

Plasma membrane protein involved in heme-iron utilization $\quad 1.75$

Cell wall protein; putative GPI-anchor $\quad 1.56$

Mycelial surface antigen precursor $\quad 2.19$

$\begin{array}{ll}\mathrm{PH} \text {-regulated antigen } & 1.58\end{array}$

Repressed by TUP1 protein 5

Histone $\mathrm{H}_{4}$

Histone $\mathrm{H}_{2} \mathrm{~B} \quad 1.86$

$\begin{array}{ll}\text { Transcriptional activator } & 1.54\end{array}$

Ferric reductase transmembrane component $\quad 1.55$

Putative glucose transporter $\quad 1.85$

Putative MFS transporter $\quad 1.56$

Carboxylic acid transporter protein $\quad 1.90$

Acetyl-coenzyme-A hydrolase $\quad 1.54$

Transcription is regulated by Nrg1p and Mig1p $\quad 1.75$

Mycelial surface antigen $\quad 1.87$

Down-regulated by Rim101p at ph 8

Fatty acid elongase required for sphingolipid formation $\quad 1.63$

High-mobility protein $1 \quad 2.34$

$\begin{array}{ll}\text { Putative beta-mannosyltransferase } & 1.66\end{array}$

Myo-inositol-1-phosphate synthase $\quad 1.66$

Similar to Saccharomyces cerevisiae Vtc1p $\quad 1.61$

Caspofungin repressed; biofilm induced $\quad 1.78$

RNA splicing protein $\quad 1.75$

Nonhistone chromosomal protein related to HMG1 2.07

Opaque-phase specific protein OP4, precursor $\quad 1.67$

Suppressor of PAB1 (by homology) $\quad 1.65$

$\begin{array}{ll}\text { Polyubiquitin } & 1.54\end{array}$

Asparagine and glutamine permease $\quad 0.52$

General amino acid permease (by homology) $\quad 0.61$

Agglutinin-like protein $\quad 0.54$

Putative beta-mannosyltransferase $\quad 0.62$

$\begin{array}{ll}\text { Hyphally regulated protein } & 0.62\end{array}$

Similar to cell wall proteins; induced in core stress response $\quad 0.58$

Putative GPI-anchored protein of unknown function $\quad 0.64$

$\begin{array}{ll}\text { Cell wall protein; putative GPI anchor } & 0.31\end{array}$ 
PIR1

orf19.220

Putative cell wall protein of the PIR family

Dehydrogenase

$\begin{array}{ll}\text { ADH1 } & \text { orf19.3997 } \\ \text { ADH2 } & \text { orf19.5113 } \\ \text { ADH5 } & \text { orf19.2608 } \\ \text { FDH11 } & \text { orf19.7600 } \\ \text { GAP1 } & \text { orf19.6814 } \\ \text { IFE2 } & \text { orf19.5288 } \\ \text { LYS12 } & \text { orf19.2525 }\end{array}$

Drug resistance

$\begin{array}{ll}\text { CRD1 } & \text { orf19.4784 } \\ \text { CDR4 } & \text { orf19.5079 } \\ \text { QDR1 } & \text { orf19.8138 }\end{array}$

\section{Glycolisis}

CDC19

ENO1

FBA1

PDC11

PDC12

PFK1

PFK2

TPI1

TPS2

orf19.3575

orf19.395

orf19.4618

orf19.2877

orf19.4608

orf19.3967

orf19.6540

orf19.6745

orf19.3038

Hexose transport

HGT8

HGT6

orf19.2021

orf19.2020

HGT7

orf19.2023

\section{Oxidase/peroxidase}

GPX2

SOD22

orf19.85

orf19.7111.1

Transcription factor

$\begin{array}{ll}\text { CPH2 } & \text { orf19.1187 } \\ \text { CTA24 } & \text { orf19.4054 } \\ \text { FCR1 } & \text { orf19.6817 } \\ \text { HAC1 } & \text { orf19.2432 } \\ \text { TYE7 } & \text { orf19.4941 } \\ \text { ZCF20 } & \text { orf19.11621 } \\ \text { Transporter } & \\ \text { ATP1 } & \text { orf19.6854 } \\ \text { ENA22 } & \text { orf19.6070 } \\ \text { IFC1 } & \text { orf19.3746 } \\ \text { PHO84 } & \text { orf19.655 } \\ \text { PMA1 } & \text { orf19.5383 }\end{array}$

\section{Other function}

BEL1

CMK1

COX1

$\mathrm{CRH} 11$

DAK2

DDI1

DED1

DEF1

ERG251

ERO1

HSP30

IPF18732

IPF2400

IPF85

IPF8806

IPF9955

OSM1

RHR2

RIB3

SAP9

SKS1

THI4

Alcohol dehydrogenase $\quad 0.47$

Alcohol dehydrogenase I $\quad 0.53$

Probable alcohol dehydrogenase $\quad 0.48$

Glutathione-dependent formaldehyde dehydrogenase $\quad 0.63$

Glyceraldehyde-3-phosphate dehydrogenase $\quad 0.47$

Protein described as an alcohol dehydrogenase $\quad 0.47$

Homo-isocitrate dehydrogenase $\quad 0.66$

Cu-transporting P1-type ATPase 0.56

Multidrug resistance protein $\quad 0.38$

$\begin{array}{ll}\text { Putative antibiotic resistance proteins } & 0.41\end{array}$

Pyruvate kinase $\quad 0.56$

Enolase I $\quad 0.54$

Fructose-bisphosphate aldolase $\quad 0.45$

Pyruvate decarboxylase $\quad 0.39$

Pyruvate decarboxylase I $\quad 0.56$

6-phosphofructokinase Alpha subunit $\quad 0.59$

6-phosphofructokinase Beta subunit $\quad 0.43$

Triose phosphate isomerase 0.58

Threalose-6-phosphate phosphatase $\quad 0.47$

Sugar transporter $\quad 0.30$

Sugar transporter $\quad 0.35$

Sugar transporter $\quad 0.16$

Glutathione peroxidase $\quad 0.47$

Superoxide dismutase $\quad 0.61$

Transcriptional activator of hyphal growth 0.53

Transcriptional activator $\quad 0.64$

Zinc cluster transcription factor $\quad 0.59$

Putative transcription factor $\quad 0.65$

Basic helix-loop-helix transcription factor $\quad 0.28$

Predicted zinc-finger protein of unknown function $\quad 0.64$

F1F0-atpase complex F1 alpha subunit $\quad 0.47$

P-type ATPase involved in $\mathrm{Na}^{+}$efflux $\quad 0.54$

Oligo-peptide transporter 0.56

High-affinity inorganic phosphate $/ \mathrm{H}^{+}$symporter $\quad 0.49$

Plasma membrane $\mathrm{H}^{+}$-transporting atpase 1

orf19.6906 Protein of the 40S ribosomal subunit 0.58

orf19.5911 $\quad \mathrm{Ca}^{2+} /$ calmodulin-dependent ser/thr protein kinase 0.56

$\begin{array}{lll}\text { CaalfMp08 Subunit I of cytochrome c oxidase } & 0.58\end{array}$

orf19.2706 Probable membrane protein 0.58

$\begin{array}{lll}\text { orf6.1104 Dihydroxyacetone kinase } & 0.35\end{array}$

orf19.7258 Response to DNA alkylation by homology 0.65

$\begin{array}{lll}\text { orf19.7392 } & \text { RNA helicase } & 0.64\end{array}$

$\begin{array}{lll}\text { orf19.7561 } & \text { Protein required for filamentous growth } & 0.40\end{array}$

orf19.12101 C-4 sterol methyl oxidase 0.46

$\begin{array}{lll}\text { orf19.4871 Required for protein disulfide bond formation in the ER } & 0.59\end{array}$

orf19.4526 Heat shock protein

orf19.8330 Histidine-rich glycoprotein precursor 0.43

$\begin{array}{lll}\text { orf19.6816 } & \text { Putative aldehyde reductase } & 0.43 \\ & \text { Stmilarto Saccharemyces } & 0.58\end{array}$

orf19.5334 Similar to Saccharomyces cerevisiae Tis $11 \mathrm{p} \quad 0.48$

$\begin{array}{lll}\text { orf19.9844 6-phosphofructose-2-kinase } & 0.55\end{array}$

orf19.10993 Regulated by Tsa1p

orf19.6882 Similar to Saccharomyces cerevisiae Osm1p 0.40

$\begin{array}{lll}\text { orf19.5437 DL-glycerol phosphatase } & 0.47\end{array}$

orf19.12693 3,4-dihydroxy-2-butanone 4-phosphate synthase $\quad 0.56$

$\begin{array}{lll}\text { orf19.6928 } & \text { Aspartyl proteinase } 9 & 0.63\end{array}$

$\begin{array}{lll}\text { orf19.3669 } & \text { Serine/threonine kinase } & 0.27\end{array}$

$\begin{array}{lll}\text { orf19.5986 } & \text { Thiazole biosynthetic enzyme precursor } & 0.52\end{array}$ 


\section{Genes involved in carbohydrate metabolism and energy generation}

Because polyamine catabolism has been reported to be involved in the regulation of energy and glucose metabolism in a mouse model [368], the analysis of the microarray data was directed towards discovering whether polyamine is involved in the energy and glucose metabolism of a $C$. albicans odc mutant. During the yeastmode of growth, numerous genes involved in glycolysis, gluconeogenesis and carbohydrate transport (HGT8, HGT6 and HGT7) were down-regulated. Oral administration of spermidine has been reported to result in the precocious expression of a carbohydrate-transporter mRNA and protein in the proximal and distal regions of the small intestine [505]. The findings reported here are the first results reported regarding the effect of polyamines on the expression of glucose transporter-related genes under low putrescine levels in C. albicans. However, a highers percentage of genes from the glycolytic pathway, encoding transcription factors and transporters showed a greater than 1.5-fold decrease during the yeast mode of growth including down-regulation of the hexose transporters (as mentioned above), small-molecule transporters (ATP1, PHO84 and PMA1).

FRE5, a predicted ferric reductase that is induced by the CCAAT-binding factor, along with the induction of Hap43p [15,29,259], of carbohydrate transporters (JEN1 and HGT14) and of a membrane transporter (orf19.7336) homologue of $S$. cerevisiae, AZR1 were the main up-regulated genes. ENA22, a homologue of ScENA5, which appears to encode a transporter involved in the uptake of sodium [504], was down-regulated. Our results suggest that lower levels of polyamines caused a significant and marked shift in the down-regulation of genes involved in metabolism and energy generation during the yeast-form growth.

\section{Regulation of other genes categories}

As shown in Table 4.1, the gene expression level was increased in four genes encoding components of histones, HTB1, HTA1, HHF21 and HHF2. The expression of genes involved in RNA processing including SBP8 homologue of $S$. 
cerevisiae LSM1 [314], IPF12381 homologue of S. cerevisiae SGN1 [509] and MRS4, an RNA-splicing protein that plays a role in chromosome rearrangements $[275,327]$, was also up-regulated during the yeast mode of growth. Only one transcription factor was up-regulated during the yeast-form growth -GCN4, described as the primary regulator of the transcriptional response to amino acid starvation [192]. However six transcription factors -CPH2, CTA24, FCR1, HAC1, TYE7 and ZCF20- were down-regulated. Cph2p regulates hyphal development in C. albicans, because the cph2/cph2 mutant strain showed medium-specific impairment of both hyphal development and induction of hypha-specific genes [262]. The other differentially expressed genes encoding for cell wall proteins (e.g ALS1, BMT4, HYR1, PGA26, PGA31, PIR1) (among them PGA10, PGA45, PGA7, PRA1 and RBT5 were up-regulation), dehydrogenases (ADH1, FDH11, IFE2 and LYS12), drug resistance controlling proteins (CDR1, CDR4 and QDR1) and oxidase/peroxidises (GPX2 and SOD3). These results suggest that polyamine level controls a broad range of gene functions.

\section{Transcriptome of odc mutant during the yeast-hypha transition}

The effect of low and high levels of polyamines was analized during the yeasthypha transition. Our analysis showed 1981 genes to be altered by comparison of both levels of putrecine, with 653 genes (32.96\%) having unknown functions. Genes expressed differentially are listed in Supplementary Tables 4.3 and 4.4. The genes with the highest- and lowest-fold expression during the three time periods of hyphal transition (15, 60 and $180 \mathrm{~min})$ were SPE2 and ECE1, encoding for an adenosylmethionine decarboxylase precursor and cell-elongation protein, respectively. Approximately 21 genes involved in the production of cell wall proteins and 15 genes controlling cell polarity and filament formation showed lower accumulation of their transcripts during the above-mentioned three times of yeasthypha transition, whereas 7 genes encoding the cell wall proteins and none related to cell polarity and filament formation showed an increase in their transcript levels during the same period (Table 4.2). 
Table 4.2 Distribution of differentially expressed genes during the three times (15, 60 and $180 \mathrm{~min}$ ) of yeast-hypha transition into functional categories in the $C$. albicans odc/odc. Growth was carried out in Lee medium supplemented with $0.01 \mathrm{mM}$ and $10 \mathrm{mM}$ of puterescine.

\begin{tabular}{|c|c|c|c|}
\hline $\begin{array}{l}\text { Gene } \\
\text { Name }\end{array}$ & Accession no & Description (CandidaDB) & Fold regulation \\
\hline
\end{tabular}

Down-regulated genes

$15 \mathrm{~min} \quad 60 \mathrm{~min} \quad 180 \mathrm{~min}$

Acetyl-coenzyme

ACC1

orf19.7466

ACS1

Acetyl-coenzyme-A carboxylase

orf19.1743 Acetyl-coenzyme-A synthetase

ACS2

orf19.1064

POT13

orf19.2046

Acetyl-coenzyme-A synthetase

Acetyl-CoA C-acyltransferase

0.41

0.47

$\begin{array}{lll}0.43 & 0.58 & 0.56\end{array}$

Adenine biosynthesis

ADE13 orf19.3870

ADE17 orf19.492

Enzyme of adenine biosynthesis

Enzyme of adenine biosynthesis

$\begin{array}{lll}0.48 & 0.21 \quad 0.44\end{array}$

Amino acid permease

$\begin{array}{ll}\text { AGP1 } & \text { orf19.8784 } \\ \text { CAN2 } & \text { orf19.111 } \\ \text { CAN5 } & \text { orf19.3641 } \\ \text { HNM3 } & \text { orf19.2587 } \\ \text { IPF4580 } & \text { orf19.6522 } \\ \text { MAL31 } & \text { orf19.3981 } \\ \text { Cell Wall } & \\ \text { AAF1 } & \\ \text { ALS3 } & \text { orf19.7436 } \\ \text { BGL22 } & \text { orf19.2355 } \\ \text { CHS5 } & \text { orf19.7339 } \\ \text { DPM1 } & \text { orf19.807 } \\ \text { ECE1 } & \text { orf19.5073 } \\ \text { ECM17 } & \text { orf19.3374 } \\ \text { ECM33 } & \text { orf19.4099 } \\ \text { FGR6 } & \text { orf19.3010.1 } \\ \text { GLC3 } & \text { orf19.5315 } \\ \text { HYR1 } & \text { orf19.13067 } \\ \text { HWP1 } & \text { orf19.12440 } \\ \text { IPP1 } & \text { orf19.1321 } \\ \text { PGA26 } & \text { orf19.3590 } \\ \text { PGA36 } & \text { orf19.2475 } \\ \text { PGA48 } & \text { orf19.5760 } \\ \text { PGA53 } & \text { orf19.6321 } \\ \text { PGA54 } & \text { orf19.12120 } \\ \text { PGA59 } & \text { orf19.2685 } \\ \text { RBT1 } & \text { orf19.2767 } \\ \text { SUN41 } & \text { orf19.1327 } \\ & \text { orf19.3642 }\end{array}$

Asparagine and glutamine permease

$\begin{array}{lll}0.26 & 0.21 \quad 0.36\end{array}$

Amino acid permease

Amino acid permease

Choline permease

Putative allantoate permease

Maltose permease

$\begin{array}{lll}0.35 & 0.36 & 0.63 \\ 0.56 & 0.42 & 0.62 \\ 0.61 & 0.60 & 0.55 \\ 0.47 & 0.51 & 0.33 \\ 0.52 & 0.58 & 0.50 \\ 0.63 & 0.44 & 0.50\end{array}$

Adhesion mediating surface antigen

$\begin{array}{lll}0.36 & 0.37 & 0.41\end{array}$

Agglutinin like protein

Putative glucanase

Putative chitin biosynthesis protein

Protein with similarity to $S$. cerevisiae Dpm1p

Cell elongation protein

Putative sulfite reductase

Cell wall biogenesis

Protein lacking an ortholog in S. cerevisiae

1.4-glucan branching enzyme

Hyphally regulated protein

Hyphal wall protein

Inorganic pyrophosphatase (hypha cell wall)

Putative GPI-anchored protein

Putative GPI-anchored protein

Putative GPI-anchored protein

Putative GPI-anchored protein

Putative GPI-anchored protein

Putative GPI-anchored protein

Repressed by TUP1 protein 1

Putative cell wall beta-glucosidase

$\begin{array}{lll}0.04 & 0.02 & 0.0\end{array}$

$\begin{array}{lll}0.64 & 0.42 & 0.33\end{array}$

$\begin{array}{lll}0.55 & 0.65 & 0.63\end{array}$

$\begin{array}{lll}0.37 & 0.28 & 0.46\end{array}$

$\begin{array}{lll}0.02 & 0.01 & 0.13\end{array}$

$\begin{array}{lll}0.63 & 0.57 & 0.62\end{array}$

$\begin{array}{lll}0.40 & 0.39 & 0.54\end{array}$

$\begin{array}{lll}0.51 & 0.38 & 0.55\end{array}$

$\begin{array}{lll}0.60 & 0.36 & 0.44\end{array}$

$\begin{array}{lll}0.35 & 0.07 & 0.18\end{array}$

$\begin{array}{lll}0.03 & 0.01 & 0.05\end{array}$

$\begin{array}{lll}0.59 & 0.20 & 0.36\end{array}$

$\begin{array}{lll}0.49 & 0.29 & 0.29\end{array}$

$\begin{array}{lll}0.31 & 0.27 & 0.26\end{array}$

$\begin{array}{lll}0.53 & 0.58 & 0.49\end{array}$

$\begin{array}{lll}0.49 & 0.45 & 0.59\end{array}$

$\begin{array}{lll}0.20 & 0.08 & 0.33\end{array}$

$\begin{array}{lll}0.43 & 0.24 & 0.44\end{array}$

$\begin{array}{lll}0.35 & 0.22 & 0.34\end{array}$

$\begin{array}{lll}0.24 & 0.18 & 0.25\end{array}$

Cell polarity and filament formation

$\begin{array}{ll}\text { ACT1 } & \text { orf19.5007 } \\ \text { ARF3 } & \text { orf19.1702 } \\ \text { ARP3 } & \text { orf19.2289 } \\ \text { BET3 } & \text { orf19.5817 } \\ \text { CDC12 } & \text { orf19.3013 } \\ \text { CDC23 } & \text { orf19.6437 } \\ \text { CHC1 } & \text { orf19.3496 } \\ \text { COF1 } & \text { orf19.953.1 } \\ \text { GDI1 } & \text { orf19.7261 } \\ \text { IPF1022 } & \text { orf19.4579 } \\ \text { MHP1 } & \text { orf19.461 } \\ \text { RDI1 } & \text { orf19.5968 } \\ \text { SEC24 } & \text { orf19.12194 } \\ \text { TEM1 } & \text { orf19.10519 } \\ \text { TPM2 } & \text { orf19.6414.3 }\end{array}$

GTP-binding protein of the ARF family

Actin related protein

Fusion of ER to Golgi transport vesicles

Septin

A anaphase-promoting complex component

Clathrin heavy chain

Protein described as cofilin

GDP dissociation inhibitor

Similar to Saccharomyces cerevisiae Erv29p

Protein similar to $S$. cerevisiae Mhp1p

Rho GDP dissociation inhibitor

Component of COPII coat of ER-Golgi vesicles

GTP-binding protein of the RAS superfamily

Tropomyosin

Dehydrogenase

ADH1

orf19.3997

$\mathrm{ADH} 2 \quad$ orf19.5113

ADH5 orf19.2608

GAP1
Alcohol dehydrogenase

Alcohol dehydrogenase

Probable alcohol dehydrogenase

Glyceraldehyde-3-phosphate dehydrogenase
0.43

0.24

0.40

$\begin{array}{lll}0.08 & 0.06 & 0.13 \\ 0.15 & 0.18 & 0.27 \\ 0.42 & 0.17 & 0.14 \\ 0.19 & 0.04 & 0.06\end{array}$




$\begin{array}{ll}\text { HOM6 } & \text { orf19.2951 } \\ \text { IDH1 } & \text { orf19.4826 } \\ \text { IDP1 } & \text { orf19.5211 } \\ \text { IDP2 } & \text { orf19.3733 } \\ \text { URA1 } & \text { orf19.4836 }\end{array}$

Ergoestrol biogenesis

$\begin{array}{ll}\text { DAP1 } & \text { orf19.489 } \\ \text { ERG1 } & \text { orf19.406 } \\ \text { ERG24 } & \text { orf19.1598 } \\ \text { ERG251 } & \text { orf19.12101 }\end{array}$

ERG251

orf19.12101

\section{Glycolysis}

CDC19

ENO1

FAB1

FBA1

GPM1

HXK2

PDC11

PDI1

PFK1

PFK2

PGK1

PGM2

TPI1

orf19.3575

orf19.395

orf19.9088

orf19.4618

orf19.903

orf19.8176

orf19.2877

orf19.5130

orf19.3967

orf19.6540

orf19.3651

orf19.10359

orf19.6745

Hexos transport

HGT6

HGT7

HGT12

orf19.2020

orf19.2023

orf19.3668

\section{Lysine biosynthesis}

$\begin{array}{ll}\text { LYS1 } & \text { orf6.1158 } \\ \text { LYS12 } & \text { orf19.2525 } \\ \text { LYS7 } & \text { orf19.4449 } \\ \text { LYS9 } & \text { orf19.7448 }\end{array}$

\section{Mitochondria}

$\begin{array}{ll}\text { CPR3 } & \text { orf19.1552 } \\ \text { GCV3 } & \text { orf19.5006 } \\ \text { HRD3 } & \text { orf19.1191 } \\ \text { MAE1 } & \text { orf19.3419 } \\ \text { MDM12 } & \text { orf19.6900 } \\ \text { MIS11 } & \text { orf19.2364 } \\ \text { POR1 } & \text { orf19.1042 } \\ \text { YHB1 } & \text { orf19.3707 }\end{array}$

Respiration and electron transport

ATP1

COX13

COX3A

COX3B

COX4

CUP5

IPF6566

NAD6

PMA1

STF2

VMA1

VAM6

orf19.6854

orf19.1467

CaalfMp04

CaalfMp15

orf19.1471

orf19.5886

orf19.6873.1

CaalfMp02

orf19.5383

orf19.2107.1

orf19.1866

orf19.1567

\section{TCA cycle}

LSC1

LSC2

OSM1

orf19.3358

orf19.1860

orf19.6882

\section{Transcription factor}

$\begin{array}{ll}\text { GCF1 } & \text { orf19.400 } \\ \text { IPF29 } & \text { orf19.5975 } \\ \text { IPF9826 } & \text { orf19.11621 } \\ \text { TIP120 } & \text { orf19.6729 } \\ \text { TYE7 } & \text { orf19.4941 } \\ \text { ZCF4 } & \text { orf19.1227 }\end{array}$

Homoserine dehydrogenase

0.58

0.61

0.49

0.30

0.38

0.36

0.39

0.32

0.22

Dihydroorotate dehydrogenase

0.29

0.50

0.45

0.57

0.64

0.39

Regulation of ergostreol biosynthesis

$\begin{array}{lll}0.44 & 0.28 & 0.41\end{array}$

Squalene epoxidase

$\mathrm{C}-14$ sterol reductase

C-4 sterol methyl oxidase

$\begin{array}{lll}0.42 & 0.44 & 0.51\end{array}$

$\begin{array}{lll}0.31 & 0.27 & 0.42\end{array}$

$0.34 \quad 0.16 \quad 0.29$

Pyruvate kinase

Enolase I

$\begin{array}{lll}0.27 & 0.12 & 0.28\end{array}$

$0.06-0.19$

$\begin{array}{llll}\text { Fructose-bisphosphate aldolase } & 0.10 & 0.06 & 0.16\end{array}$

$\begin{array}{llll}\text { Phosphoglycerate mutase } & 0.37 & 0.16 & 0.30\end{array}$

Hexokinase II

Pyruvate decarboxylase

Protein disulfide-isomerase precursor

$\begin{array}{lll}0.44 & 0.38 & 0.36\end{array}$

$\begin{array}{lll}0.20 & 0.11 & 0.14\end{array}$

6-phosphofructokinase Alpha subunit $\quad \begin{array}{llll}0.23 & 0.26 & 0.33\end{array}$

6-phosphofructokinase beta subunit $\quad 0.20 \quad 0.16 \quad 0.19$

Phosphoglycerate kinase

Phosphoglucomutase

Triose phosphate isomerase

$\begin{array}{lll}0.11 & 0.06 & 0.15\end{array}$

$\begin{array}{lll}0.49 & 0.23 & 0.16\end{array}$

$\begin{array}{lll}0.30 & 0.16 & 0.27\end{array}$

Sugar transporter

Sugar transporter

Hexose transporter

$\begin{array}{lll}0.57 & 0.30 & 0.39\end{array}$

$\begin{array}{lll}0.45 & 0.46 & 0.57\end{array}$

$\begin{array}{lll}0.57 & 0.44 & 0.46\end{array}$

Saccharopine dehydrogenase

Homo-isocitrate dehydrogenase

Chaperone of superoxide dismutase Sod1p

0.49

0.38

0.47

0.23

0.38

$0.66 \quad 0.30$

0.42

Lysine biosynthesis

0.41

Peptidylprolyl isomerase Mitochondrial

0.66

0.32

0.41

Glycine decarboxylase Subunit $H$

Involved in HMG-COA reductase degradation

Mitochondrial malic enzyme

$0.18 \quad 0.43$

$\begin{array}{lll}0.54 & 0.61 & 0.53\end{array}$

$0.40 \quad 0.56$

nvolved in mitochondrial inheritance

$0.39-0.47-0.39$

$\begin{array}{llll}\text { Mitochondrial C1-tetrahydrofolate synthase } & 0.56 & 0.30 & 0.51\end{array}$

$\begin{array}{llll}\text { Mitochondrial outer membrane porin } & 0.58 & 0.13 & 0.22\end{array}$

Flavohemoglobin (by homology)

$\begin{array}{lll}0.19 & 0.18 & 0.22\end{array}$

Protein similar to alpha subunit of ATP synthase

0.39

Prome-c oxidase chain via

$0.16 \quad 0.29$

Subunit III of cytochrome c oxidase $\quad 0.39$

Subunit III of cytochrome c oxidase

$0.43 \quad 0.54$

$0.47 \quad 0.38$

$0.50-0.38$

Cytochrome-c oxidase

Vacuolar $\mathrm{H}^{+}$-ATPase

$0.43-0.51$

Subunit 6 of NADH:ubiquinone oxidoreductase $\quad 0.47 \quad 0.57 \quad 0.65$

Plasma membrane $\mathrm{H}^{+}$-transporting ATPase $1 \quad 0.47 \quad 0.52 \quad 0.40$

$\begin{array}{llll}\text { ATP synthase regulatory factor } & 0.34 & 0.11 & 0.13\end{array}$

Vacuolar ATPase V1 domain subunit G $\quad 0.61 \quad 0.36 \quad 0.53$

$\begin{array}{llll}\text { Vacuolar carboxypeptidase } Y & 0.26 & 0.36 & 0.38\end{array}$

$\begin{array}{llll}\text { Succinate-coa ligase / synthetase } & 0.63 & 0.28 & 0.46\end{array}$

$\begin{array}{llll}\text { Succinate-coa ligase beta subuni } & 0.61 & 0.30 & 0.49\end{array}$

Flavoprotein subunit of fumarate reductase $\quad \begin{array}{lll}0.16 & 0.18 & 0.33\end{array}$

Protein that binds the regulatory region HWP1 $0.58 \quad 0.53 \quad 0.59$

$\begin{array}{llll}\text { Putative transcription factor } & 0.29 & 0.28 & 0.36\end{array}$

Predicted zinc-finger protein of unknown function $0.34 \quad 0.40 \quad 0.35$

$\begin{array}{llll}\text { TBP-binding transcription regulator } & 0.61 & 0.54 & 0.47\end{array}$

$\begin{array}{llll}\text { Basic helix-loop-helix transcription factor } & 0.49 & 0.52 & 0.48\end{array}$

$\begin{array}{llll}\text { Putative transcription factor } & 0.51 & 0.44 & 0.44\end{array}$ 


\section{Transport}

$\begin{array}{ll}\text { CTR1 } & \text { orf19.3646 } \\ \text { FET54 } & \text { orf19.4215 } \\ \text { HOL4 } & \text { orf19.12021 } \\ \text { PHO84 } & \text { orf19.1172 }\end{array}$

Other function

55 genes

Unknown function

33 genes

Up-regulated genes

$\begin{array}{ll}\text { Cell wall } & \\ \text { BMS1 } & \text { orf19.2504 } \\ \text { ECM1 } & \text { orf19.5299 } \\ \text { MNN11 } & \text { orf19.2927 } \\ \text { MNN4 } & \text { orf19.10399 } \\ \text { PGA31 } & \text { orf19.5302 } \\ \text { PHR2 } & \text { orf19.13500 } \\ \text { PIR1 } & \text { orf19.220 }\end{array}$

DNA-directed RNA polymerase

RPB8

RPC25

RPC40

RPC53

RPC82

PAF1

orf19.6340

orf19.6314

orf19.443

orf19.3564

orf19.2715

orf19.2847

orf19.3613

Mitochondrial ribosomal protein

RSM7

MRPS12

MRP1

MRP10

MRP17

MRP20

MRP7

MRPL17

MRPL40

MRPS28

MRT4

YML6

orf19.4018

orf19.2438

orf19.1662

orf19.947

orf19.3350

orf19.7203

orf19.585

orf19.484

orf19.2520

orf19.12996

orf19.7019

Nucleolar protein

$\begin{array}{ll}\text { ENP1 } & \text { orf19.5507 } \\ \text { ENP2 } & \text { orf19.6686 } \\ \text { NOP1 } & \text { orf19.3138 } \\ \text { NOP14 } & \text { orf19.5959 } \\ \text { NOP15 } & \text { orf19.7050 } \\ \text { NOP2 } & \text { orf19.501 } \\ \text { NUP133 } & \text { orf19.3552 } \\ \text { NUP84 } & \text { orf19.1298 }\end{array}$

Ribosomal protein

RPF1

orf19.10184

RPL23A

RPN4

RPS27A

RRP42

RRP6

RRP8

RRP9

RRS1

IMP3

orf19.3504

orf19.1069

orf19.413.1

orf19.5039

orf19.58

orf19.3630

orf19.2830

orf19.6014

orf19.7488

RNA helicase

DBP2

DBP3

DBP8

DBP9

CHR1

orf19.171

orf19.12334

orf19.6652

orf19.3393

orf19.3756
Copper transport protein

Protein similar to multicopper ferroxidase

Member of major facilitator superfamily

Inorganic phosphate transport protein

$\begin{array}{lll}0.50 & 0.49 & 0.31 \\ 0.34 & 0.20 & 0.35 \\ 0.46 & 0.49 & 0.52 \\ 0.22 & 0.49 & 0.41\end{array}$

orf19.2650.1

Membrane protein involved in bud site selection

Involved in cell wall biosynthesis

2.18

Mannosyltransferase complex component

Protein required for mannosylphosphorylation 2.83

Cell wall protein; putative GPI anchor 2.62

Putative cell wall protein of the PIR family $\quad 3.52$

3.75

3.32

2.73

2.48

1.98

2.18

2.29

2.36

2.00

2.59

1.79

1.78

2.23

1.71

DNA-directed RNA polymerase I, II, III $\quad 2.06$

DNA-directed RNA polymerase I, II, III $\quad 1.69$

DNA-direcred RNA polymerase III

1.69
2.11

2.11
2.29

1.75

DNA-directed RNA polymerase III

DNA-directed RNA polymerase III

DNA-directed RNA polymerase II

2.26

1.96

2.62

3.25

$1.82 \quad 1.77$

$2.15 \quad 2.22$

$2.42-2.89$

$2.43 \quad 2.00$

$2.32 \quad 2.16$

$1.80 \quad 2.43$

Putative mitochondrial ribosomal protein S7 2.11

Putative mitochondrial ribosomal protein S12 1.91

$1.72 \quad 1.85$

Mitochondrial ribosomal protein

Mitochondrial ribosomal protein $\quad 1.92$

Mitochondrial ribosomal protein

1.92

Similar to Saccharomyces cerevisiae Mrp20p 1.67

Mitochondrial ribosomal protein yml2 precursor $\quad 2.49$

Ribosomal protein of the large subunit $\quad 1.85$

Putative mitochondrial ribosomal protein $\quad 1.94$

Ribosomal protein

Required for mRNA decay

Ribosomal protein, Mitochondrial

Essential nuclear protein

3.82

Mutation confers resistance to 5-fluorocytosine 2.68

Fibrillarin

Mutation confers resistance to 5-fluorocytosine $\quad 2.87$

$\begin{array}{llll}\text { A nucleolar ribosome biogenesis factor } & 3.16 & 4.17 & 2.93\end{array}$

Nucleolar protein $\quad 1.70$

Nuclear pore protein

$3.26-2.92$

Nuclear pore protein

$1.65-1.79$

$1.99 \quad 1.67$

Maturation of 5.8S rRNAfrom tricistronic rRNA $\begin{array}{lll}2.35 & 2.22 & 2.15\end{array}$

Ribosomal protein L23.e

$26 \mathrm{~S}$ proteasome subunit

Ribosomal protein S27.e

rRNA processing protein

Involved in 5.8S rRNA processing

$1.61 \quad 2.18$

$\begin{array}{ll}1.61 & 2.18 \\ 1.67 & 3.50\end{array}$

$1.67 \quad 1.89$

$2.62 \quad 2.69$

$2.93 \quad 2.09$

$2.91 \quad 2.68$

Similar to $S$. cerevisiae RRP8

$\begin{array}{llll}\text { U3 small nucleolar ribonucleoprotein } & 3.20 & 4.40 & 3.41 \\ \text { Regular } & 1.99 & 2.27 & 2.57\end{array}$

$\begin{array}{llll}\text { U3 small nucleolar ribonucleoprotein } & 1.83 & 2.36 & 2.06\end{array}$
ATP-dependent RNA helicase of DEAD box family1.81

ATP-dependent RNA helicase 2.26

DEAD box protein ATP-dependent RNA helicase 1.77

Dead box helicase

DEAD-box ATP-dependent RNA helicase
3.52

5.22

2.24 


\begin{tabular}{|c|c|c|c|c|c|}
\hline DRS1 & orf19.7635 & ATP dependent RNA helicase & 2.07 & 2.54 & 1.85 \\
\hline HAS1 & orf19.11444 & ATP-dependent RNA helicase & 1.79 & 2.67 & 2.15 \\
\hline MRH4 & orf19.3481 & Putative ATP-dependent RNA helicase & 1.65 & 2.18 & 2.58 \\
\hline MTR4 & orf19.8915 & RNA Helicase & 1.56 & 1.90 & 2.00 \\
\hline RRP3 & orf19.7546 & RNA-dependent ATPase, Helicase & 1.79 & 2.13 & 1.64 \\
\hline SUV3 & orf19.4519 & ATP-dependent RNA helicase & 2.26 & 2.41 & 2.10 \\
\hline \multicolumn{6}{|c|}{ Transcription factor } \\
\hline ACE2 & orf19.6124 & Putative transcription factor & 2.38 & 1.92 & 1.72 \\
\hline BCR1 & orf19.723 & Transcription factor & 2.10 & 3.03 & 2.92 \\
\hline BRE1 & orf19.976 & Putative transcription factor & 1.65 & 1.57 & 1.71 \\
\hline CAP1 & orf19.9191 & Transcriptional acivator & 1.78 & 2.65 & 1.78 \\
\hline CTA8 & orf19.4775 & Activates transcription in 1-hybrid assay & 1.81 & 2.68 & 1.76 \\
\hline DAL81 & orf19.3252 & Transcriptional activator & 1.79 & 2.14 & 1.65 \\
\hline GCN4 & orf19.1358 & Transcriptional activator & 3.20 & 3.72 & 3.64 \\
\hline INO2 & orf19.7539 & Transcriptional activator & 2.26 & 2.33 & 1.83 \\
\hline IPF6857 & orf19.1757 & Putative transcriptional regulator & 1.93 & 2.46 & 1.94 \\
\hline NRG1 & orf19.7150 & Transcriptional repressor & 2.59 & 3.44 & 1.86 \\
\hline RMS1 & orf19.10177 & Putative transcriptional regulator & 2.83 & 3.47 & 2.28 \\
\hline SFU1 & orf19.4869 & Transcriptional regulator of iron-responsive genes & s 2.12 & 1.90 & 1.98 \\
\hline TAF19 & orf19.5174 & TBP-associated factor by homology & 1.77 & 1.73 & 1.82 \\
\hline TBP1 & orf19.1837 & Transcription initiation factor & 1.89 & 2.29 & 2.67 \\
\hline WOR2 & orf19.5992 & Transcriptional regulator of white-opaque switch & 1.83 & 2.00 & 2.00 \\
\hline ZCF3 & orf19.1168 & Predicted zinc-finger protein of unknown function & 2.72 & 3.10 & 2.90 \\
\hline ZPR1 & orf19.3300 & Zinc finger protein & 1.87 & 4.28 & 3.80 \\
\hline \multicolumn{6}{|c|}{ Translation initiation factor } \\
\hline CDC95 & orf19.9378 & Translation initiation factor 6 & 2.23 & 3.42 & 3.14 \\
\hline GCD11 & orf19.4223 & Translation initiation factor elF2 & 1.70 & 2.88 & 1.84 \\
\hline GCD7 & orf19.825 & Translation initiation factor eilFb subunit & 1.82 & 2.03 & 2.05 \\
\hline NIP1 & orf19.4635 & Translation initiation factor subunit & 1.90 & 2.25 & 1.96 \\
\hline SUA5 & orf19.7088 & Translation initiation protein & 1.69 & 2.20 & 1.94 \\
\hline SUI1 & orf19.8867 & Translation initiation factor 3 & 2.35 & 1.68 & 2.61 \\
\hline SUI3 & orf19.7161 & Translation initiation factor elF2 beta subunit & 1.66 & 1.84 & 2.38 \\
\hline TIF1 & orf19.3324 & Translation initiation factor & 3.15 & 2.86 & 2.22 \\
\hline \multicolumn{6}{|c|}{$\begin{array}{l}\text { Other Function } \\
104 \text { genes }\end{array}$} \\
\hline \multicolumn{6}{|c|}{ Unknown Function } \\
\hline
\end{tabular}

Consistent with the phenotipic pattern of mutant cells during the yeast-hypha transition at low levels of polyamine $(0.01 \mathrm{mM})$, as reported previously by Herrero et al [188], several hypha-specific genes were down-regulated. Among these, we highlight the down-regulation of ECE1 [32], RBT1 [37], HWP1 [419], DDR48 [261], PHR1 [370], HYR1 [370] and ALS3 during the three times of yeast hypha transition. We have also identified down-regulation of several other genes encoding cell wall proteins, putative GPI-anchored proteins (PAG26, PAG36, PAG48, PAG53, PAG54 and PAG59) and up-regulation of MNN11, MNN4, PGA31, PHR3 and PIR1. Other categories of down-regulated genes at the three times of yeast-hypha transition represented functional molecules as diverse as genes involved in acetyl coenzyme A (ACC1, ACS1, ACS2 and POT13), adenine biosynthesis proteins (ADE13 and $A D E 17)$, amino acid permeases (e.g. AGP1, CAN2 and CAN5), proteins controlling cell polarity and filament formation ( $A C T 1, A R F 3$ and $A R P 3)$, dehydrogenases (ADH1, ADH2 and HOM6), proteins of lysine biosynthetic pathway (LYS1, LYS7 and LYS12) and ergosterol biogenesis (ERG1, ERG24 and ERG251) (Table 4.2). 
Also we found down-regulation of genes such as GCF1, IPF29, TIP120, TYE7, ZCF4 and ZCF20, coding for transcriptional factors.

\section{Expression of metabolic genes}

About 13 genes involved in the glycolytic pathway includindig genes encoded enzymes that are required for both glycolysis and gluconeogenesis showed a decline in their transcript levels during the three times of yeast-hypha transition, whereas none of the genes of this pathway were up-regulated (Table 4.2). It is therefore probable that glycolisis and gluconeogenesis are down-regulated in the odc mutant.

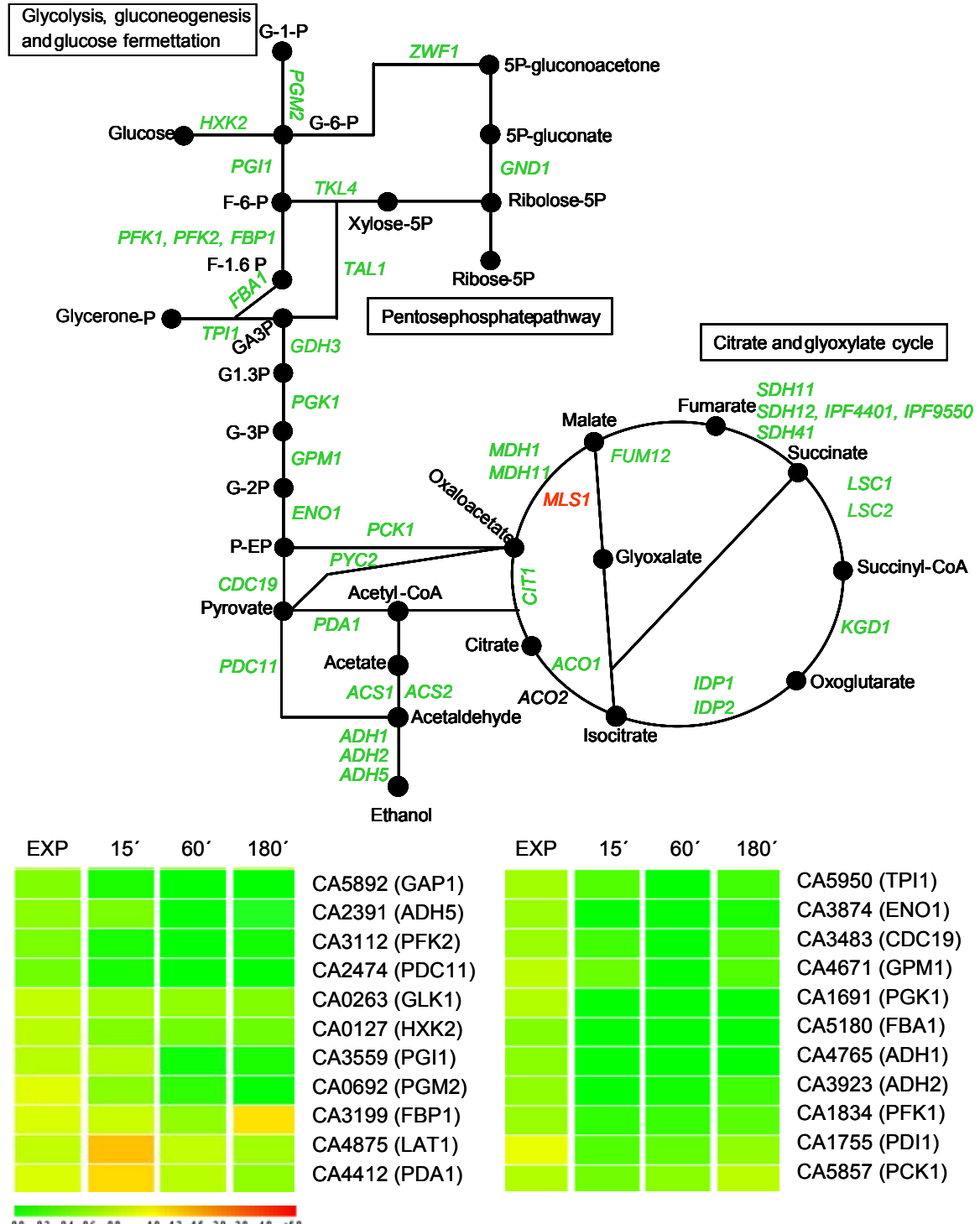

Figure 4.2 Transcriptional regulations of genes involved in carbohydrate metabolism by $C$. albicans odc/odc mutant during yeast mode of growth and during the yeast-hypha transition. Representative genes responsible for specific cellular functions are grouped and their regulation at different times (15, 60 and $180 \mathrm{~min}$ ) of hypha development are listed. Red (up-regulated genes), green (down-regulated), and black (un-changed genes) names represent the CandidaDB 
Under hypha-inducing conditions, numerous genes having predicted roles in mitochondria functions showed increased expression. These included a large number of proteins with structural roles in mitochondrial organisation and biogenesis, such as mitochondrial ribosomal proteins (Table 4.2). Figure 4.3 shows the expression patterns of genes involved in electron transport and respiration during the first, second and third periods of incubation and include a several of enzymes required for the tricarboxylic acid cycle (Table 4.2) and components required for electron transfer were down-regulated.

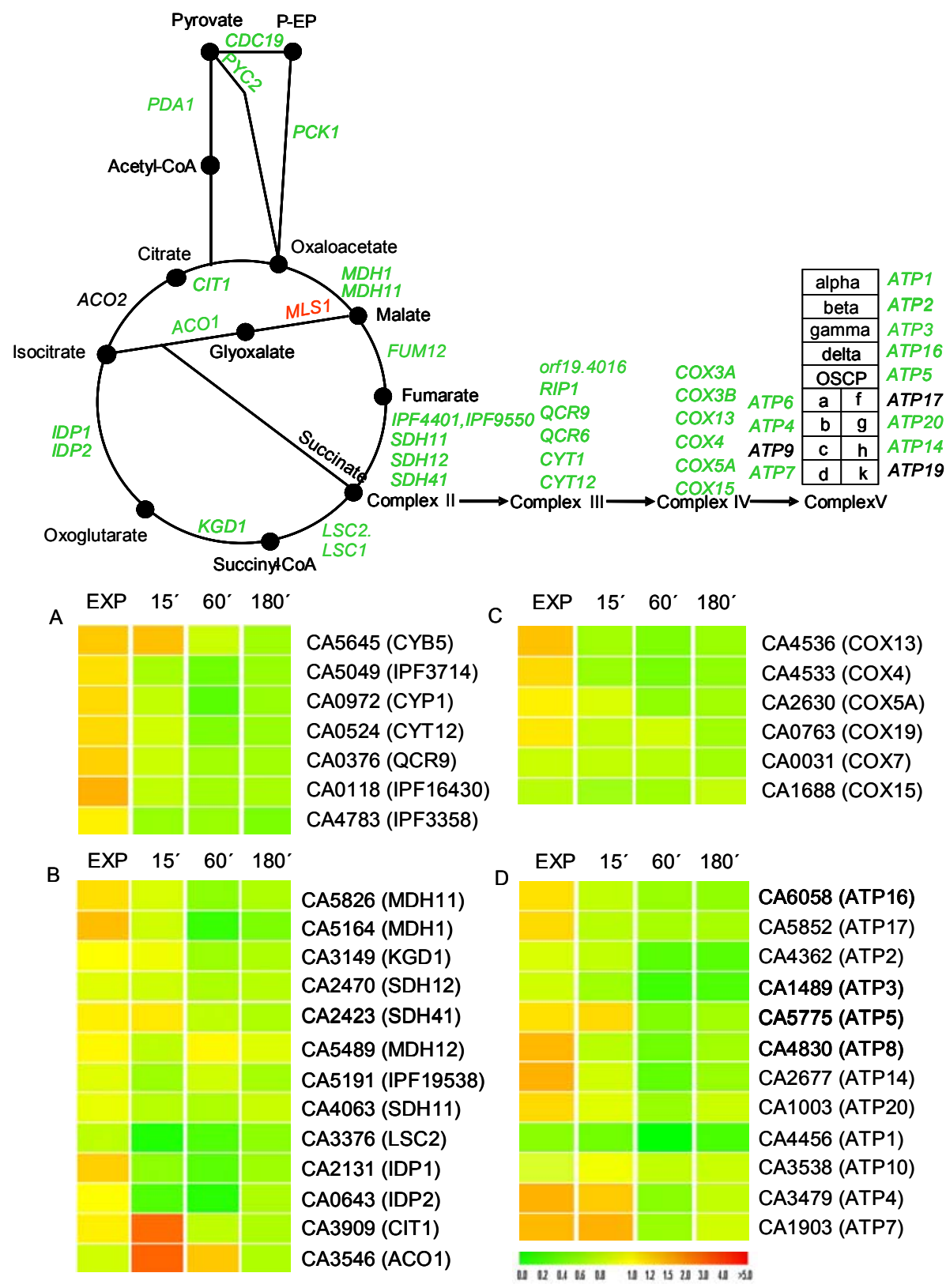

Figure 4.3 Transcriptional regulations of genes involved in respiration by $C$. albicans odc/odc mutant during yeast mode of growth and during the yeast-hypha transition in Lee medium. Representative genes responsible for specific cellular functions are grouped and their regulation at different times (15, 60 and $180 \mathrm{~min})$ of hypha development are listed. Red (up-regulated genes), green (down-regulated), and black (un-changed genes) names represent the CandidaDB.Transcript levels of corresponding complex III (A), TCA pathway (B), complex IV (C) and complex V (D). 
Overall, these results suggest that electron transport and respiration are repressed in the odc mutant strain during the yeast-hypha transition. Lower metabolic activity of the mutant cell at the three times of yeast-hypha transition due to downregulation of genes involved in carbohydrate metabolism (glycolysis and hexose transport) and amino acid permease (AGP1, CAN2, CAN5, HNM3, IPF4580 and MAL31) can reflect a lower respiratory activity. Our results give new clues to understand the role of polyamines in $C$. albicans and further work is needed to characterise the effect of polyamines on electron transport and respiration in $C$. albicans. Inhibition of both ODC activity and putrescine uptake causes mitochondrial damage and loss of mitochondria in Leishmania promastigote cell [483], which is in agreement with the down-regulation of electron transport and energy generation-related transcripts at low polyamine levels.

\section{Other transcripts}

The transcriptional profiling analysis showed up-regulation of 17 of genes encoding for transcription factors during the three times of yeast-hypha transition (Table 4.2). These genes were ACE2, a putative transcription factor involved in the regulation of morphogenesis producing its deletion a hyperfilamentous phenotype [232]; BCR1, a transcription factor required for biofilm formation but not for hyphal growth [226,354,377]; $B R E 1$, a putative transcription factor with the $\mathrm{C}_{3} \mathrm{HC}_{4}$ zinc-finger DNA-binding motif, transposon mutation of which affects filamentous growth [354,477]; CAP1, a transcription factor of the AP-1 family, involved in oxidative stress response and multidrug resistance [3,4,494,495,536]; Cta8p, a protein that activates transcription in one-hybrid assay in $S$. cerevisiae and is transcriptionally activated by Mnl1p under weak-acid stress [228,377]; DAL81, a predicted zincfinger protein of unknown function that has similarity to $S$. cerevisiae Dal81p, which is involved in the regulation of nitrogen-degradation genes [298,544], GCN4, a transcriptional activator of general amino acid control response [472]; INO2, a transcriptional activator that may be involved in the regulation of ribosomal protein genes [198]; CA2262, a putative transcription factor with the zinc-finger DNAbinding motif, which transcription is up-regulated in clinical isolates from HIV+ patients with oral candidiasis [354,529]; NRG1, a transcriptional repressor involved in regulation of hyphal genes [38,330]; TAF19, a transcription factor involved in regulation of transcription during the $\mathrm{G} 1$ phase of mitotic cell cycle [518]; TBP1, a 
transcription initiation factor that binds to the TATA box sequence [273]; WOR2, a transcriptional regulator of the white-opaque switching that is required for maintenance of opaque state [545]; ZCF3, a predicted zinc-finger protein of unknown function [298]; ZPR1, a protein with putative zinc finger that is regulated by Gcn4p [390]; and SFU1, a transcriptional regulator of iron-responsive genes [259].
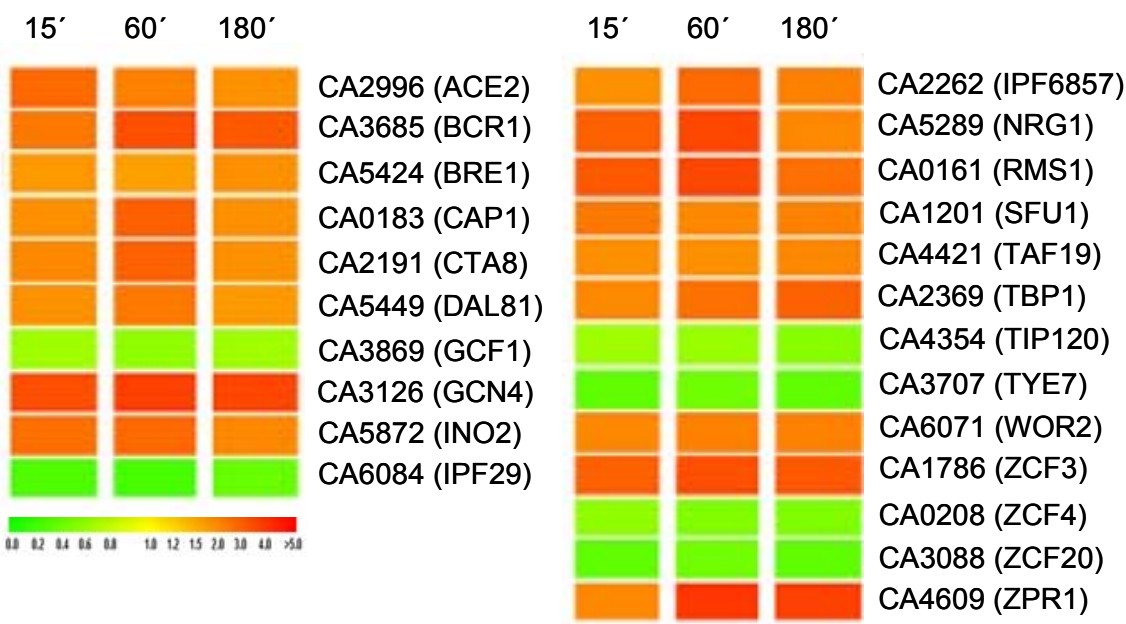

Figure 4.4 Transcriptional regulations of genes encoding transcription factors during three times of incubation by $C$. albicans odc/odc mutant.

Among the down-regulated transcription factors, we have found GCF1, a protein that binds to the regulatory region in the HWP1 promoter and regulates a hyphaspecific gene [235]; CA6084, a putative transcription factor; CA3088, a predicted zinc-finger protein of unknown function; TIP120, a TBP-binding transcription regulator; TYE7, a basic helix-loop-helix transcription factor; and ZCF4, a putative transcription factor (Figure 4.4).

Other up-regulated genes included proteins such as DNA-directed RNA polymerase (e.g RBP5, RBP8 and RPC25) (Figure 4.5A), nucleolar proteins (e.g ENP1, ENP2 and NOP1) (Figure 4.5B), ribosomal proteins (e.g RPL23, RPS27A and RRS1) (Figure 4.5C), RNA helicases (DBP2, DBP3 and DBP8) (Figure 4.5D) and translation initiation factors (CDC95, GCD11 and GCD7) (Figure 4.5E). We also detected that the expression of several genes encoding for mitochondrial ribosomes were increased in all the three times of hypha transition (Figure 4.5F). Currently, very little information regarding the behaviour of these genes is available; nevertheless, the regulation of these groups of genes by polyamines is interesting, because it supports our hypothesis about the possibility of polyamines being involved in the global regulation of gene expression. 

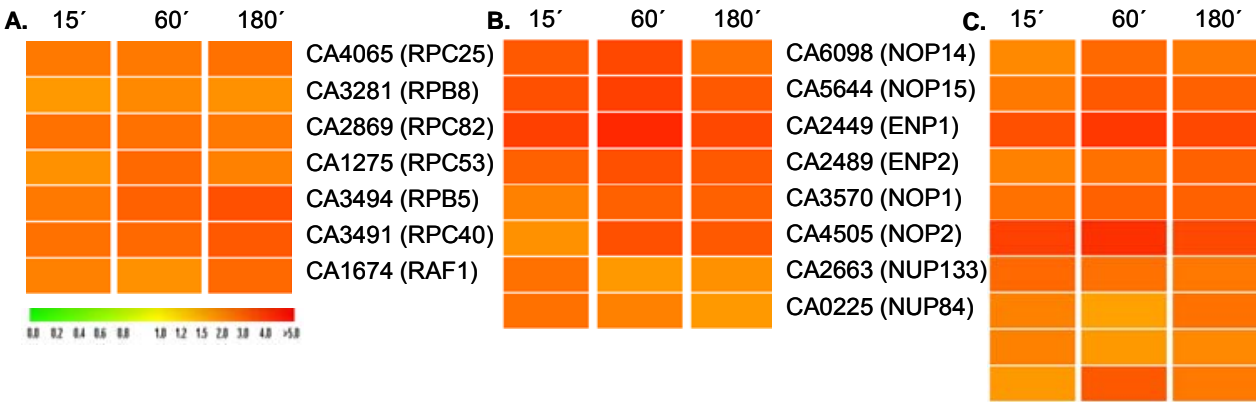

CA5833 (IMP3) CA1277 (RRP8) CA2545 (RRP9) CA2992 (RRS1) CA1695 (RRP42) CA2854 (RPN4) CA0599 (RPF1) CA2454 (RPL23A) CA2130 (RPS27A) CA2593 (RRP6)

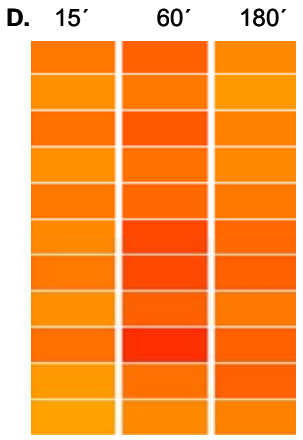

CA6029 (DRS1)
CA5957 (RRPS3
CA1002 (CHR1)
CA4092 (DBP8)
CA2908 (SUV3)
CA1416 (DBP2)
CA0715 (DBP9)
CA0554 (HAS1)
CA1202 (DBP3)
CA2898 (MRH4)
CA2119 (MTR4)

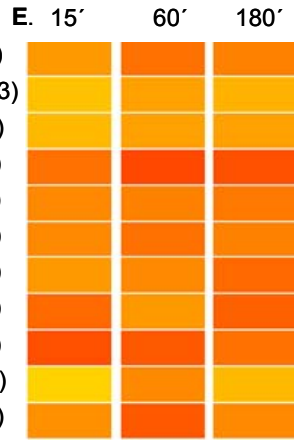

\begin{tabular}{l|l|l|l}
\multicolumn{1}{c|}{ F. $15^{\prime}$} & $60^{\prime}$ & $180^{\prime}$ \\
\hline CA5611 (SUA5) & & & \\
CA2359 (TIF35) & & & \\
CA3754 (TIF3) & & & \\
CA0323 (CDC95) & & & \\
CA4222 (GCD7) & & & \\
CA0733 (NIP1) & & & \\
CA5298 (SUI3) & & & \\
CA2357 (SUI1) & & & \\
CA2939 (TIF1) & & & \\
CA1354 (GCD6) & & \\
CA1322 (GCD11) & & \\
& & \\
& & \\
\end{tabular}

CA5405 (MRP17) CA5329 (MRP7) CA0986 (MRP20) CA3432 (MRPL17) CA0462 (MRPS28) CA5204 (YML6) CA4785 (RSM7) CA4521 (MRPL40) CA1224 (MRP10) CA3466 (MRP1) CA1914 (MRPS12) CA2283 (MRT4)

Figure 4.5 Transcriptional regulation by temperature of genes involved in DNA-directed RNA polymerase (A), nucleolar proteins (B), ribosomal proteins(C), RNA helicase (D), translation initiation factors (E) and mitochondrial ribosomes $(\mathbf{F})$ Representative genes were grouped by gene families.

\section{Common up- and down-regulated genes at 15 and 60 minutes of induction}

The analysis of the microarray data of cells obtained during the hyphal transition showed a total of 188 genes to be differentially expressed commonly between 15 and 60 min of induction: 92 genes were up-regulated and 96 were down-regulated (Supplementary Tables 4.3 and 4.4).

Among the up-regulated genes, we highlight the induction of genes encoding the rRNA processing protein ( $F A L 1, R P P 1$ and HCA4), proteins involvd in glutamate metabolism (GDH3, GNP1, PUT1 and PUT2), genes encoding stress-response proteins (HSP70, HSP78, HSP90, SSA2, SSC1, SSE1 and STI1) and four transcription factors (MIG1, a transcriptional repressor that regulates genes for utilisation of carbon sources [139,330]; SNF2, a protein involved in transcriptional regulation, orthologue of $S$. cerevisiae $S n f 2 p$, which is the catalytic subunit of the SWI/SNF chromatin remodelling complex that interacts with Swi1p [301]; STP4, a putative transcription factor with the zinc-finger DNA-binding motif; induced in core caspofungin response [91,140,354]; and TEC1, a TEA/ATTS transcription factor involved in regulation of hypha-specific genes and required for biofilm formation $[29,261,262,354])$. Among the down-regulated genes, we have detected four 
groups of genes involved in the following functional categories. Genes involved in the secretory pathway; ARF21- an ADP-ribosylation factor that is involved in intracellular transport [107,263]; CLC1 -similar to S. cerevisiae CLC1 a subunit of the major coat protein, involved in intracellular protein transport and endocytosis [425]; COP1- similar to S. cerevisiae COP1 Alpha subunit of the COPI vesiclecoatomer complex, which surrounds the transport vesicles in the early secretory pathway [145]; ERD2- similar to $S$. cerevisiae ERD2, an integral membrane protein that both binds to the HDEL motif in proteins destined for retention in the endoplasmic reticulum and that has a role in the maintenance of normal levels of ER-resident proteins [178,366,412]; ERV25 -orthologue of S. cerevisiae ERV25, a protein that forms a heterotrimeric complex with Erp1, Erp2p, and Emp24, members of the p24 family involved in endoplasmic reticulum to the Golgi transport [25,329]; ERV46 a protein localised in COPII-coated vesicles that forms a complex with Erv41p involved in the membrane-fusion stage of transport [71]; SEC16, orthologue of S. cerevisiae SEC16, a COPII-vesicle coat protein required for ER transport-vesicle budding and autophagosome formation (Sec16p is bound to the periphery of ER membranes and may act to stabilise initial COPII complexes $[150,420,459])$; SEC18, a functional homologue of S. cerevisiae Sec18p, which acts in protein transport [347]; SEC23, orthologue of $S$. cerevisiae SEC23, component of the Sec23p-Sec24p heterodimeric complex of the COPII vesicle coat, involved in ER to Golgi transport and autophagy [111,217]; SEC26, orthologue of $S$. cerevisiae SEC26, an essential beta-coat protein of the COPI coatomer, involved in ER-to-Golgi protein trafficking and maintenance of normal ER morphology [112]; SEC31, orthologue of S. cerevisiae SEC31, essential phosphoprotein component ( 150$)$ of the COPII coat of secretory pathway vesicles [398]; and SLY1, orthologue of S. cerevisiae SLY1 a hydrophilic protein involved in vesicle trafficking between the ER and Golgi [243] (Figure 4.6). The second set contains the genes involved in O-directed glycosylation (PMT2, PMT1 and PMT4). The third set of genes is implicated in the mitotic cell cycle and cell cycle control (CDC1, CDC11, CDC42 and RAS1) (Figure 4.6). And finally, three transcription factors including CAS4, a protein of RAM cell-wall integrity-signalling network with transcription factor activity that is required for hyphal growth [26,46,439]; TFC1, a protein with RNA polymerase III transcription factor activity [297]; and UME6, a transcription factor that is required for wild-type hyphal extension [22]. 

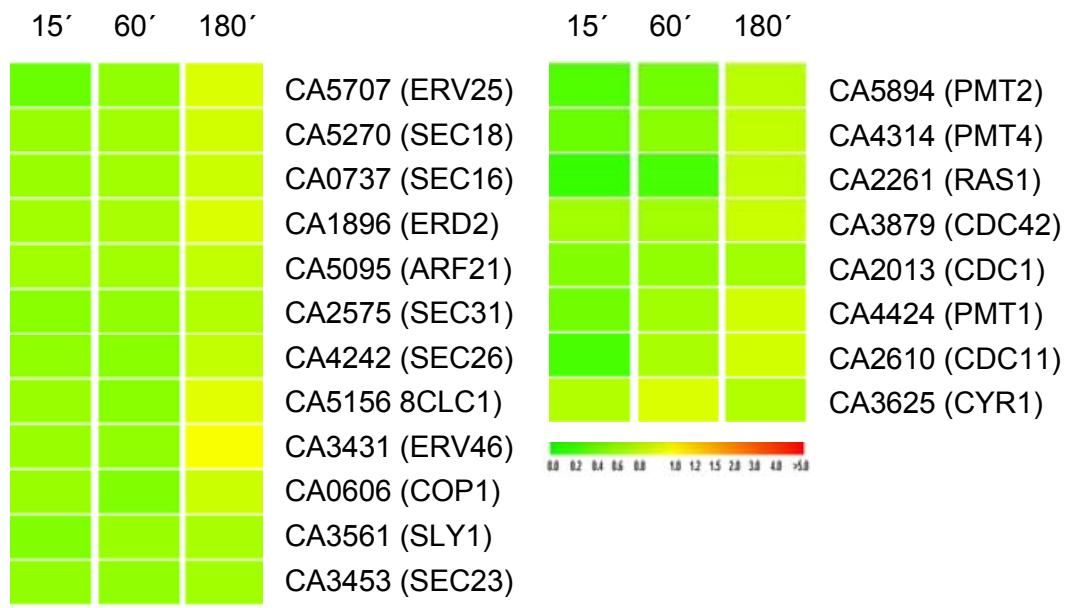

Figure 4.6 Transcriptional regulations of genes involved in secretory pathway and signal transduction during the three times of incubation by $C$. albicans odc/odc mutant.

\section{Common up- and down-regulated genes at 60 and 180 minutes of induction}

Of the total up- and down-regulated genes between 60 and $180 \mathrm{~min}$ of incubation, we observed common differential expression of 267 genes; 130 were up-regulated and 137 genes were down-regulated.

Of the 130 ORFs, four genes (MRPL23A, MRPL36, MRPL49 and MRPS5) encode proteins involved in mitochondrial ribosomal proteins, five (GCD2, GCD14, PRT, TIF5 and YIF2) encode proteins involved in translation initiation factors, three genes (RPA12, RPA49 and RPB4) code for proteins involved in DNA-directed RNA polymerase II and 10 genes (IPF12778, IPF7998, NIP7, NOP4, NOP58, RPL15B, $R R P 1, R R P 45, R S A 2$ and SIK1) encode proteins involved in ribosomal protein. We detected four genes encoding proteins involved in transcription factors, namely, CAS5 -a putative transcription factor that regulates response to cell wall damage and is required for wild-type transcriptional response to caspofungin [46,354]; HAP41 -a protein with transcription activator activity that is transcriptionally activated by Mnl1p under weak acid stress [39]; SUA70 -a predicted transcriptional regulator that is down-regulated during planktonic growth [196,334]; and TAF60-a transcription factor of the TFIID complex with general RNA polymerase II transcription factor activity [518]. Among the down-regulated genes, we have identified a number of genes involved in electron transport (ATP14, ATP16, ATP2, ATP3, ATP5, ATP8, COX5A, CYT12, IPF3358, MCR1, QCR6 QCR9 and VMA2) (Supplementary Table 4.4 and Figure 4.3), cell polarity (ABP1, ARC40, ARP2, 
CRN1, PFY1 and TUB2), tricarboxylic acid pathway (FUM12, LSC2, MDH1 and MDH11) (Figure 4.3) and cell wall formation (BGL21, CHT2, PGA4, PGA56 and PHR1). We observed down-regulation of three transcriptional factors, including CTA24 -a putative transcriptional activator that is down-regulated by Efg1p [108]; UME7 -a putative transcription factor with zinc-cluster DNA-binding motif, similar to $S$. cerevisiae Ume6p, which is involved in the regulation of meiotic genes [298,354,544]; and TUP1 -a transcriptional corepressor [330].

\section{Sets of genes regulated only at one incubation time}

A total of 289 genes were expressed differentially at 15 min of hypha transition, among which 124 and 165 genes were up- and down-regulated, respectively.

Expression of some categories of up-regulated, genes include those involved in nitrogen, sulphur and selenium metabolism (TRR1, UGA11, NFU1, ARG1 and GLN1), two aconitate hydratases (ACO1 and ACO2), several multidrug resistance factors (CDR1, CDR2 and QDR2) and ribosomal proteins MRPL32, MRPL11, RSM18, RPS10, MRPL3, MRPL24 and MRPL33.

The main categories of down-regulated genes correspond to those encoding cell wall proteins (ALG8, ALS2, GAA1, IFF5, MNN2, MNT2, MNT3, PGA37 and TOS1) and genes involved in metabolism of porphyrins (HEM2, HEM12 and HEM15). We have detected regulation of eight transcription factors including CTA24, a member of a family of telomere-proximal genes (a putative transcriptional activator that is down-regulated by Efg1p [108,482,529]); GAL4, a putative transcription factor with a zinc cluster DNA-binding motif [306,544]; HAC1, a putative transcription factor that is involved in unfolded protein response and control of cell morphology [354,508,539]; IPF14284, a putative transcription factor with a zinc-finger DNA-binding motif, similar to bacterial DnaJ, which is transcriptionally regulated by iron [297,354]; IPF7646, a predicted transcriptional regulator with a bZip domain [110]; and two predicted zinc-cluster proteins of unknown function ZCF1, ZCF32 [298,364] (Supplementary Tables 4.3 and 4.4). Most of these genes didn't change thier expression after 60 and 180 min of induction.

The expression of 388 genes was affected by a factor of $\geq 1.5$ at low levels of polyamines compared with growth at high polyamine levels at $60 \mathrm{~min}$; among these, 196 and 192 genes were up- and down-regulated, respectively. The main 
categories of up-regulated genes were genes belong to ribosome biogenesis (PET56, MAK21, RRN3, RRP4 and RRP43), translation initiation (GCD6, TIF35 and TIF3), RNA helicase (DBP2, DBP6, DED1, ECM16 and MSS116), and DNAdirected RNA polymerase (RPA135, RPA43 and RPO31). Five transcription factors -ARG81, a putative transcription factor with zinc-cluster DNA-binding motif, with similarity to $S$. cerevisiae Arg81p involved in the regulation of arginine-responsive genes [298,354,544]; CRZ1, a putative transcription factor and similar to $S$. cerevisiae calcineurin-regulated transcription factor $\operatorname{Crz1p}[354,360]$; MRR1, a zincfinger protein and regulator of MDR1 transcription, mutations of which cause upregulation of MDR1 (which encodes the plasma membrane multidrug efflux pump) $[113,326]$; SEF2, a putative zinc-cluster protein, whose expression is repressed by Sfu1p under high-iron conditions [294,298]; and ZMS1, a putative transcription factor with zinc-finger DNA-binding motif [354]- were also up-regulated. Genes involved in electron transport (ATP20, ATP4, ATP7, COB ,COQ2, COX15, CTM1, CYT1, IPF12564, NAD1, NCP1, RIP1, TFP1, TFP3, VMA4, VMA7 and VMA8), TCA cycle (FUM12, SDH11 and SDH12) (Figure 4.3 and Supplementary Table 4.4), ribosomal protein (RNH1, RPL28, RPL30, RPL33, RPL39, RPL9B, RPS19A, RPS21B and RPS25B), adenine biosynthesis (ADE1, ADE6, ADE12 and APT1), cell cycle and cell polarity (BUD2, BUD6 and $C D C 10)$ and two transcription factors -SUC1, a putative transcriptional regulator with $\mathrm{N}$-terminal zinc finger that regulates alpha-glucosidase in C. albicans and S. cerevisiae [233,298]; and ZCF39, a putative transcription factor with a zinc-cluster DNA-binding motif [298,354]- were down-regulated.

The expression of 288 genes was affected by a factor of $\geq 1.5$ after $180 \mathrm{~min}$, with 181 and 107 genes being up- and down-regulated, respectively. The overrepresented categories of up-regulated genes after $180 \mathrm{~min}$ were genes encoding mitochondrial ribosomal proteins (IMG1, IPF3698, MRPL15, MRPL16, MRPL28, MRPL31, MRP2 MRPL39, MRPL7, MRPS8 and MRPL9) and proteins involved in mitochondrial transport (OXA1, TOM20, TIM9, TIM44, TIM54 and $Y M C 1)$. Other group of genes include those involved in response to exposure to the cadmium ion exposure (GSH1, ZRC1 and MET14) and three transcription factors HAC1, TAF25, similar to $S$. cerevisiae TAF10, involved in RNA polymerase II transcription initiation and chromatin modification [163]; and TFA1, a protein with polyglutamate motifs and abundant Ser/Thr residues, described as a subunit of 
TFIIE, which is a basal transcription initiation factor of RNA Polymerase I [141]also were up-regulated. Some of the down-regulated genes encode proteins involved in electron transport (COX19, COX7, CYB5, GDH3 and GLT1), histones (HHF21, HTA1 and HTA3) and thiamine biosynthesis (THI4, THI6 and THI13).

\section{Genes involved in signal transduction}

Because it has been reported that $C$. albicans odc null mutant shows defects in hyphal transition at low polyamine levels [188] and that the cAMP signal pathway is a regulating system involved in the hyphal transition $[16,17,218,403]$, our analysis was focussed on genes involved in signal transduction. In our data, seven differentially expressed genes involved in signal transduction were affected and all were down-regulated at the first and second time points of hyphal transition. The transition from yeast-hypha of $C$. albicans is influenced by temperature and this signal-transduction cascade is modulated by RAS1, which controls the CAMP pathway and MAP-kinase cascade in C. albicans [265,388]. Because adenylate cyclase is a component of the cAMP pathway and it is involved in the growth of signals, RAS1 activates both cell growth and hyphal transition of $C$. albicans $[65,388]$. Down-regulation of signal transduction transcripts, such as those of RAS1, and decrease in expression of CYR1 (Figure 4.6) at two time periods of hyphal transition supplemented with $0.01 \mathrm{mM}$ of putrescine, suggested that adequate levels of polyamines are necessary for activation of the RAS-signal pathway.

\section{Discussion}

Polyamines are essential for normal cell growth, as has been shown in several studies with mutants and inhibitors of the polymine metabolic pathway $[50,59,95,101,170,195,225,493]$. However, the effect of polyamine starvation has not yet been clearly established. Some authors have reported that elongation of proteins and nucleic acids diminishes and that translation fidelity is impaired [188]. In addition, polyamines have a distributed charge, whose spacing may allow them to interact more flexibly with phosphates, DNA and RNA [188]. The biological function of polyamines in $C$. albicans has been reported previously by our group by both deletion of the $O D C$ gene and analysis of the consequences of this deletion on growth. The effects of low $(0.01 \mathrm{mM})$ and high $(10 \mathrm{mM})$ polyamine 
concentrations on the yeast-hypha transition and on several phenotypes associated with cell-wall defects have been shown [188]. The null odc mutant of $C$. albicans behaves as a putrescine auxotroph, which is in agreement with the results described for other fungi, such as S. cerevisiae [133], Neurospora crassa [100] and $U$. maydis [170]. Global transcriptome profiles for $C$. albicans odc mutant exposed to low and high polyamine levels were generated and compared both during the yeast mode of growth and during the yeast-hypha transition. Several studies have compared the effect of polyamines on yeast and mammalian cells $[58,88,171,300,475]$. Our transcriptome analysis showed that low polyamine levels affect the level of expression of 2019 genes during yeast mode of growth and during the hypha-transition conditions. The K-means clustering approach illustrated that the expression of $16.86 \%$ of these genes was similarly affected during the yeast-form growth and yeast-hypha transition (data not shown).

This suggests that cells may have developed common transcription regulatory mechanisms to accommodate all the biological functions under low polyamine levels during both yeast growth and hyphal transition. During the hyphal transition, numerous genes involved in DNA-directed RNA polymerases, nucleolar proteins, RNA helicases (a large group of enzymes that function in virtually all aspects of RNA metabolism), ribosomal proteins and translation initiation factors were upregulated at all three time points of incubation, suggesting polyamines play a role in the regulation of genes involved in protein synthesis and RNA metabolism. It has been reported that spermidine regulates protein synthesis (by stimulation at low concentrations and inhibition at high concentrations) [423]. There is a common theme in eukaryotic polyamine biology: the effect of polyamines on protein synthesis and the regulation of polyamine synthesis by translational mechanisms. Clearly, polyamines have many functions in the cell; however, protein synthesis may be particularly sensitive to the intracellular polyamine environment. Protein synthesis is more rapidly affected by polyamine depletion than RNA or DNA synthesis [197]. Over-accumulation of polyamines has been associated with an inhibition of protein synthesis [184,185], leading to decreased cell growth and eventual cell death. Increase in expression of SPE2 (adenosylmethionine decarboxylase precursor), genes involved in translation initiation factors and ribosomal proteins under low levels of polyamine after the three time periods of incubation suggests that polyamines play a role in control of mRNA translation 
initiation and ribosomal leaky scanning in $C$. albicans. Up-regulation of a number of genes that code for mitochondrial ribosomal proteins at the first, second and third time periods of incubation suggests that one of the metabolic changes caused by low polyamine levels in cells is the increase in expression of genes encoding protein synthesis, especially of mitochondrial protein synthesis. Ribosome production may exploit more than $50 \%$ of the synthetic effort of rapidly growing eukaryotic cells [142]. Therefore, by increasing ribosome synthesis, cells may be able to redirect these resources towards the defence against the post-translational effects of low polyamine levels. Among the down-regulated genes after the three time points of induction, we identified a number of genes that code for cell wall proteins, putative GPI-anchored proteins and genes have been detected previously as hypha-specific genes $[130,342,356]$, suggesting that polyamines may mediate trancriptional control of cell wall-related genes. As mentioned above, the downregulated genes encode cell wall proteins that are known to be associated with filamentous growth in C. albicans [18,32,37,131,261,419], thereby confirming the present results and are in agreement with the phenotype of the mutant strain in low levels of polyamine during hyphal transition as described previously [188]. It will be of interest to understand how and why polyamines lead to a drastic reduction in their transcripts. Several studies have compared the effects of polyamines on the transcriptome in yeast and mammalian cells. There has, however, been little overlap between the responsive genes reported in these studies and those presented in the current report. This may be because our experiment is a unique study that was carried out to investigate the role of polyamines in the dimorphism of C. albicans. Transcriptome analysis showed that the expression of genes involved in carbohydrate metabolism including genes involving in glycolysis and glucose transport were down-regulated during the yeast hypha transition. In yeast, low glucose causes a decrease in the level of cAMP, with concomitant low PKA activity $[222,457,546]$. Furthermore, there was a decrease in the expression of the maltase gene MAL31 (maltose permease), which metabolises maltose into glucose $[85,320]$. The combination of decrease in sugar production and transport and decrease in expression of TYE7 may lead to a glucose level that would limit PKA activity. This will ultimately lead to blocking of hypha outgrowth. In addition, we observed down-regulation of most of the genes encoding glycolysis enzymes during the yeast mode of growth, which is in agreement with the growth-rate pattern 
of mutant cell in low level of polyamine as previously reported [188]. Among the other transcripts that showed down-regulation, genes involved in the biosynthesis of acetyl coenzyme A, amino acid permeases, cell polarity and filament formation, dehydrogenases, ergosterol biogenesis, respiration and electron transport (Table 4.2) were the predominant ones. However, down-regulation of most of the genes involved in cell polarity and filament formation, respiration and electron transport categories were observed after one and two times of hypha development. These results suggest that low levels of polyamines cause a significant and marked reduction in gene expression, especially for the genes categories denoted previously and that, this down-regulation is connected with the morphogenetic switch.

The mutant cells show decreased respiration during hyphal transition, which is accompanied by down-regulation of genes encoding subunits III of cytochrome c oxidase, NADH ubiquinone oxidoreductase and isocitrate dehydrogenase transcripts at the second and third time periods of hyphal transition, indicating a low metabolic status of the $C$. albicans mutant cells during the yeast-hypha transition. Overall, these results provide strong evidence that polyamines control the metabolic status, especially the carbohydrate metabolism (glycolysis), in C. albicans, which may contribute significantly towards maintaining homeostasis, electron transport and production of ATP.

Consistent with the post-translational role attributes of polyamines, several transcripts involved in the secretory pathway and genes related to protein-oglycosylation were down-regulated at 15 and $60 \mathrm{~min}$ of hyphal transition (Figure 4.6). The changes observed in both the cellular metabolism and intracellular transport in the mutant cells are obviously not the results of a single mode of action of polyamines. However, it is clear that transcription/translation of a number of genes is coordinated with polyamine-mediated changes in the metabolite profiles and other transcriptional profiles in the mutant cells at $0.01 \mathrm{mM}$ of putrescine, suggesting that activation and sustenance of transcription is one mode by which polyamines regulate $C$. albicans metabolism during both growth in the yeast form and during the yeast-hypha transition.

In animal cells, higher polyamine concentrations regulate transcription either by acting as a switch between different coactivators without altering the state of the chromatin [295] or by affecting histone acetylation/deacetylation [194]. 
Furthermore, a post-transcriptional role for polyamines has been shown in the down-regulation of animal genes [276], whereas a certain group of Escherichia coli genes is controlled by the diamine putrescine at the translational level [528]. In the latter case, it was suggested that putrescine enhances transcription of a group of genes by increasing the level of transcription factors. Polyamine-mediated modulation of protein synthesis may involve, among other things, structural changes in RNA, stimulation of ribosomal assembly, tRNA formation [215] or a frameshift during translation into protein, which is dependent on a Shine-Dalgarnolike sequence in E. coli [190].

Regulation of gene expression by polyamines can also occur through their effects on the structure of elF5A or oligomerisation of nucleosomal arrays [94]. It is of interest to note here that the levels of eIF5 transcripts (TIF5) and other translation initiation factors as mentioned above were increased at all the three time points of incubation. Analyses of differentially expressed genes during the yeast-hypha transition identified the down-regulation of several genes encoding enzymemediated signal transduction that may be involved in regulation of hyphal transition. These include CDC42 (control of cell division), RAS1 (GTP-binding protein), RHO3 (GTP-binding protein of the rho family), SAR1 (GTP-binding protein of the ARF family) and RSR1 (GTP-binding protein) at the first and second time points of incubation. Decreases in expression of RAS1 and of CYR1 genes (Figure 4.6) during hyphal transition suggest that inactivation of PKA due to low cAMP levels will ultimately lead to the blocking of hyphal outgrowth. The PKA pathway coordinates post-translational regulation of a variety of proteins, such as the key enzymes of glycolysis and gluconeogenesis [303,345], suggesting that pathways involved in the sensing and transduction of signals are preferentially affected during hyphal transition. 


\section{Analysis of Candida albicans mutant lacking the histone acetyltransferases, GCN5 and HAT1}

\section{Abstract}

The HAT1 gene of Candida albicans was disrupted in a gcn5 background to investigate the role of the two histone acetyltransferases, GCN5 and HAT1, in growth, morphogenesis, and pathogenicity. When cultured in liquid medium Lee $\mathrm{pH}$ 6.8 at $37^{\circ} \mathrm{C}$, the gcn5, hat 1 double mutant failed to form hypha on all liquid and solid media assayed, except in embedded medium. Other phenotypes observed included sensitivity to Congo red, Hygromycin, Fluconazole, Itraconazole, and Caffeine. Whole-genome microarray analysis of the gcn5, hat1 double mutant was used to identify differential expression of genes during the yeast mode of growth. The expression of a total of 521 genes was found to be altered in which 247 genes were upregulated and 274 were down-regulated. The main categories of differentially expressed genes were those corresponding to oxidative stress response, oxidase/peroxidases, dehydrogenases, transport activity, cell wall proteins, amino acid metabolism, ergoestreol biosynthesis, glycolysis pathway, and aerobic respiration. About $32 \%$ of the differentially regulated genes included genes encoding products not yet classified for the functional attributes.

Last, an attenuated virulence of the gcn5, hat1 mutant under the usual statistical infection protocol was shown.

\section{Introduction}

Candida albicans, the most frequently isolated fungal pathogen in humans, is dimorphic; it switches between the budding yeast form and the filamentous hyphal or pseudohyphal form. This transition is induced by many different environmental cues including high temperature, neutral $\mathrm{pH}$, serum, etc. Dimorphism is thought to contribute to the virulence of Candida [79,148,210,408,421,445]. Histone acetyltransferases (HATs) are a class of enzymes regulating histone acetylation, which has been identified in several cancers. In particular, imbalance in histone acetylation can lead to changes in chromatin structure and transcriptional misregulation of genes that are involved in the control of proliferation, cell-cycle progression, and differentiation [47]. The histone acetyltransferases, GCN5 and HAT1, are proteins with important roles in the regulation of the gene expression of C. albicans, although their functions have not yet been widely investigated. In the 
present work we show that deletion of the GCN5 and HAT1 as double mutant like both simple mutants is associated with a difference between the growth rate of the double mutant and CAI4 liquid medium at $28^{\circ} \mathrm{C}$. Morphologically the double mutant, like both simple mutants, is very different to the CAI4 strain. The main phenotype of the double mutant is the inability to switch from the yeast to the filamentous form in liquid media containing serum or at $37^{\circ} \mathrm{C}$ and in all the solid media assayed.

\section{Materials and methods}

\section{Strains, medium and growth conditions}

Table 5.1 strain used in this study

\begin{tabular}{|c|c|c|c|}
\hline Strains & Genotype & Parental strain & Reference \\
\hline \multicolumn{4}{|c|}{ C. albicans strains } \\
\hline SC5314 & Clinical isolate & & [149] \\
\hline CAI4 & ura3::imm434/ura3::imm434 & CAF2-1 & [132] \\
\hline CAMR & ura3::imm434/URA3 & CAI4 & (Rodriguez and Dominguez, 2005) \\
\hline RGL4 & $\begin{array}{l}\text { ura3::imm434/ura3::imm434 } \\
\text { hat1::hisG/hat1::hisG }\end{array}$ & CAI4 & (Degano and Dominguez, 2000) \\
\hline AHM1 & $\begin{array}{l}\text { ura3::imm434/ura3::imm434 } \\
\text { gcn5::hisG/gcn5::hisG } \\
\text { hat1::hisGURA3hisG /HAT1 }\end{array}$ & RDG4 & This work \\
\hline AHM2 & $\begin{array}{l}\text { ura3::imm434/ura3::imm434 } \\
\text { gcn5::hisG/gcn5::hisG } \\
\text { hat1::hisG/HAT1 }\end{array}$ & AHM1 & This work \\
\hline AHM3 & $\begin{array}{l}\text { ura3::imm434/ura3::imm434 } \\
\text { gcn5::hisG/gcn5::hisG } \\
\text { hat1::hisGURA3hisG/hat1 }\end{array}$ & AHM2 & This work \\
\hline AHM4 & $\begin{array}{l}\text { ura3::imm434/ura3::imm434 } \\
\text { gcn5::hisG/gcn5::hisG } \\
\text { hat1::hisG/hat1::hisG }\end{array}$ & AHM3 & This Work \\
\hline HAV1 & $\begin{array}{l}\text { ura3::imm434/URA3 } \\
\text { gcn5::hisG/gcn5::hisG } \\
\text { hat1::hisG/hat1::hisG }\end{array}$ & AHM4 & This work \\
\hline RDG4 & $\begin{array}{l}\text { ura3::imm434/ura3::imm434 } \\
\text { gcn5::hisG/gcn5::hisG }\end{array}$ & CAI4 & Degano and Dominguez, 2000) \\
\hline \multicolumn{4}{|c|}{ E. coli strain } \\
\hline $\mathrm{DH} 5 \alpha$ & \multicolumn{3}{|c|}{$\begin{array}{l}\text { F- } \text { (80lacZ } \triangle M 15, \Delta(l a c Z Y A-a r g F), ~ U 169, \text { deoR, [175] } \\
\text { recA1, endA1, hsdR17,.phoA, supE44, (rk-, mk+), } \\
\text { gyrA96, } 4 \text {-thi-1, gyrA96 relA, }\end{array}$} \\
\hline \multicolumn{4}{|l|}{ Plasmid } \\
\hline pJBR3 & \multicolumn{2}{|c|}{$\begin{array}{l}\text { Hat1 promoter and terminator flanked } \\
\text { in URA3-marked pSNC1 vector }\end{array}$} & (Degano and Dominguez, 2000) \\
\hline
\end{tabular}


Escherichia coli strain was grown at $37^{\circ} \mathrm{C}$ in LB medium supplemented with $100 \mu \mathrm{g}$ of ampicillin per $\mathrm{ml}$ for plasmid selection. C. albicans strains used in this study, listed in Table 5.1, were cultured at $28^{\circ} \mathrm{C}$ in YEPD. The solid and liquid media used were as have been described in Chapter III for inducing the yeast-hypha transition.

\section{Media for integrative transformation}

Gene deletion was performed using the "Ura-blaster" protocol as previously described [132]. Ura ${ }^{+}$transformants were streaked on MM plates; single colonies were picked and grown in $5 \mathrm{ml}$ YNB medium. Genomic DNA was extracted for Southern analysis and diagnostic PCR. Counter selection of Ura- segregants was done on YNB medium containing 5-fluoro-orotic acid (5-Foa) and $0.2 \mathrm{mM}$ uridine.

Table 5.2 Primes used in this study

\begin{tabular}{llll}
\hline Primer & Sequence 5' $\mathbf{~} \mathbf{3}^{\prime}$ & Primer & \multicolumn{1}{c}{ Sequence 5' $\mathbf{3}^{\prime}$} \\
\hline URA1 & GGATACTATCAAACAAGAGG & RD17 & GGTGACAAACTAGTTCAATGC \\
URA2 & AATGCTGGTTGGAATGCTTA & RD18 & GCTTATCAAGCTTTGGAAG \\
IP2 & TTACAATCAAAGGTGGTCC & RD32 & GGCAATCACATCAAGACT \\
IP3 & GGTACAGTTGTTCCTCACA & RD33 & TGTTCAACTTCAAGTCTC \\
\hline
\end{tabular}

The DNA manipulations, disruption of the HAT1 gene and analysis of nucleic acids have been carried out as described in Chapter III.

\section{Results}

\section{Construction of the C. albicans gcn5, hat1 double mutant}

Ura-blaster cassette was used to construct a CaHAT1 deleted strain in a gcn5 mutant background (Figure 5.1). The pJBR3 plasmid used in this study was constructed by Degano and the $C$. albicans gcn5 mutant was transformed using the protoplast method. After transformation the C. albicans RDG4 (gcn5-Ura ${ }^{-}$) strain with pJBR3, four colonies were obtained. PCR analysis (not shown) indicated that two transformants contained a proper deletion of one allele of the CaHAT1 gene. The positive transformants were streaked on 5-Foa medium. The colonies that grew on these plates were checked by PCR for the absence of the $C$. albicans URA3 gene. One of the positive colonies was transformed again with the pJBR3 construct. In this second round of transformation, five colonies were screened, and two hat1 transformants were found with both alleles deleted as is shown by Southern blot (Figure 5.1.B). 
A.
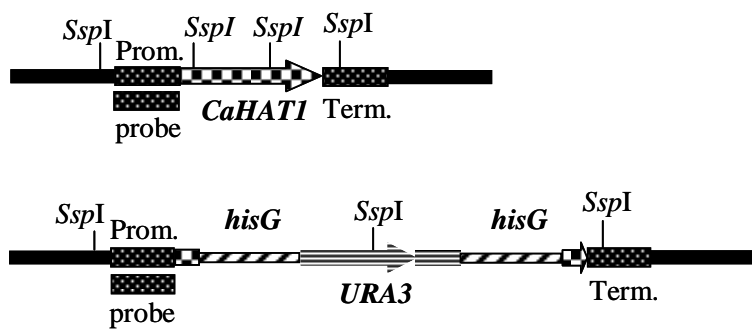

$\begin{array}{cc}\text { SspI } \\ \text { Prom. } \\ \text { probe } & \text { hisG }\end{array}$

B.

kb

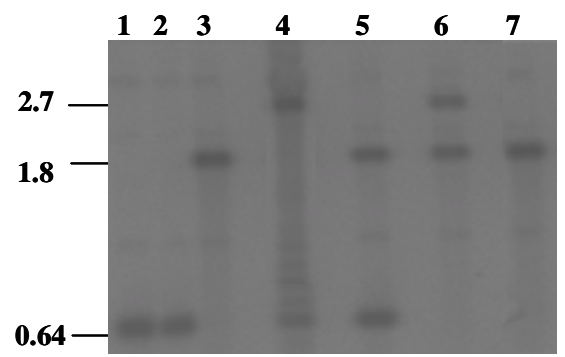

Figure 5.1 Deletion of CaHAT1 alleles (A) Structure of different alleles.

The HAT1 gene and the alleles disrupted by the hisG-URA3-hisG cassette or by hisG alone are shown. (B) Southern blot analysis of genomic DNA was performed with the following strains digested with Sspl. Lane 1, CAI4 (GCN5/GCN5; HAT1/HAT1)

Lane 2, RDG4 ( $\triangle g c n 5::$ hisG/Agcn5::hisG;HAT1/CaHAT1)

Lane 3, RGL4 (GCN5/GCN5; $\Delta$ hat1::hisG/Ahat1::hisG)

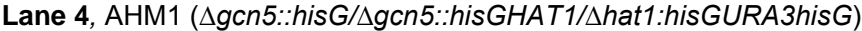

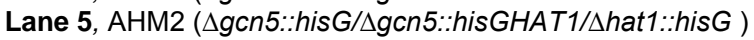

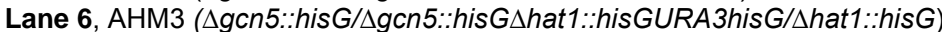

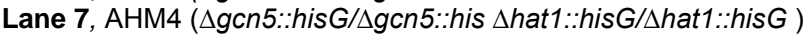

The $459 \mathrm{bp}$, fragment was used as a probe.

\section{Phenotype of the double mutant cells}

Morphologically, the double mutant like both simple mutants is very different from CAl4 strain as show in Figure 5.2. The culture of the gcn5, hat1 double mutant in YEPD liquid medium like both simple mutants is mainly composed of a mixture of spherical cells and short chains of elongated, aberrantly shaped cells attached to one another.

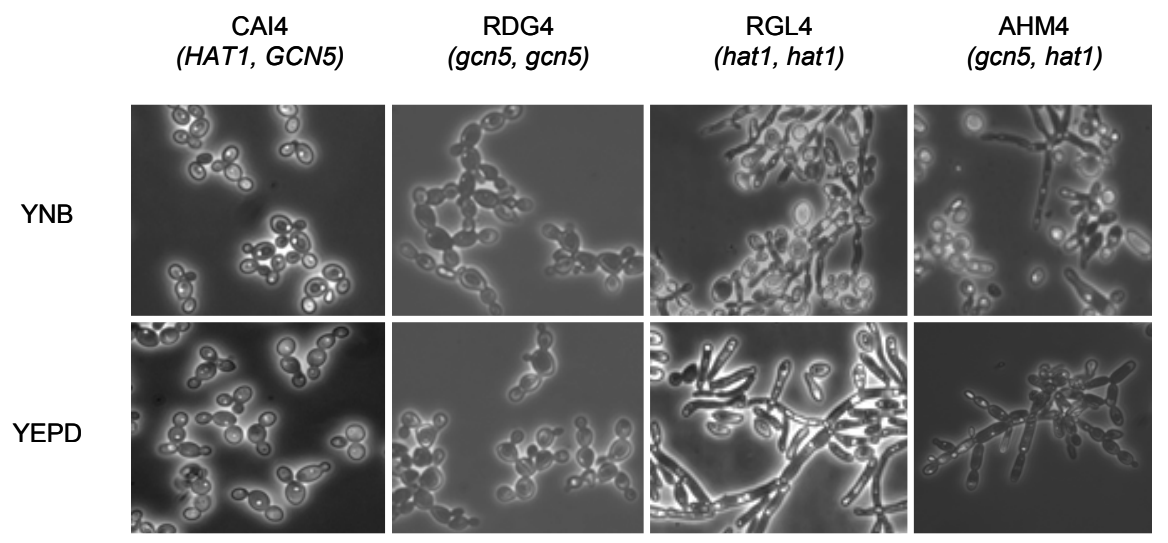

Figure 5.2 Cellular morphology of CAI4, RDG4, RGL4 and AHM4 strains in YNB and YEPD medium

The growth rate of the gcn5, hat1 strain was 1.5-fold slower than that of CAl4 strain (Figure 5.3). This result suggested that deletion of hat1 compensates partially the growth defect of the gcn5 mutant under standard conditions. Another distinctive 
feature of the gcn5, hat1 mutant was the color; it was whiter than the parental and CAl4 strains (data not show).

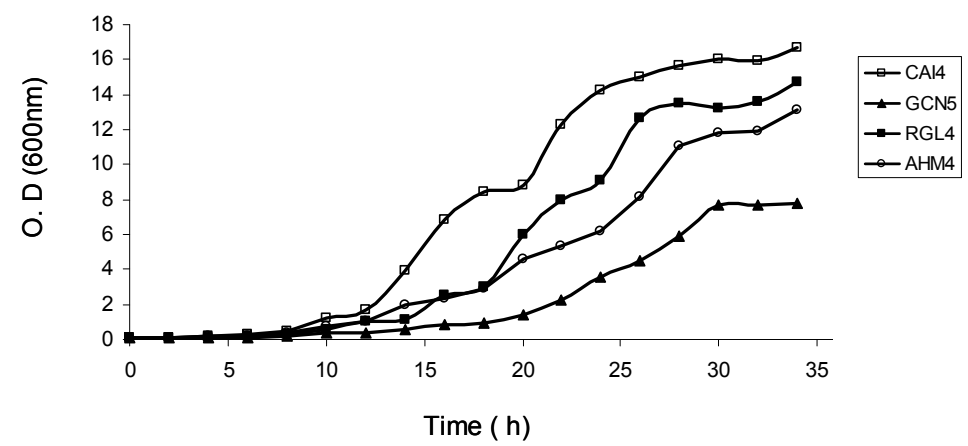

Figure $\mathbf{5 . 3}$ Growth curves of the mutant strains. Strains were pre-grown on rich medium containing $1 \%$ glucose and inoculated at an O.D at $600 \mathrm{~nm}$ of 0.1 YEPD. Growth was monitored every two hours over a period of $34 \mathrm{~h}$ by measuring the optical density of the cultures.

\section{Behavior of the double mutant strain in solid media}

In order to determine whether the absence of $C$. albicans Gcn5p and Hat $1 p$ is involved in hyphal morphogenesis, we examined the ability of CAI4, double and both single mutants to undergo hyphal transition under several conditions on solid media.

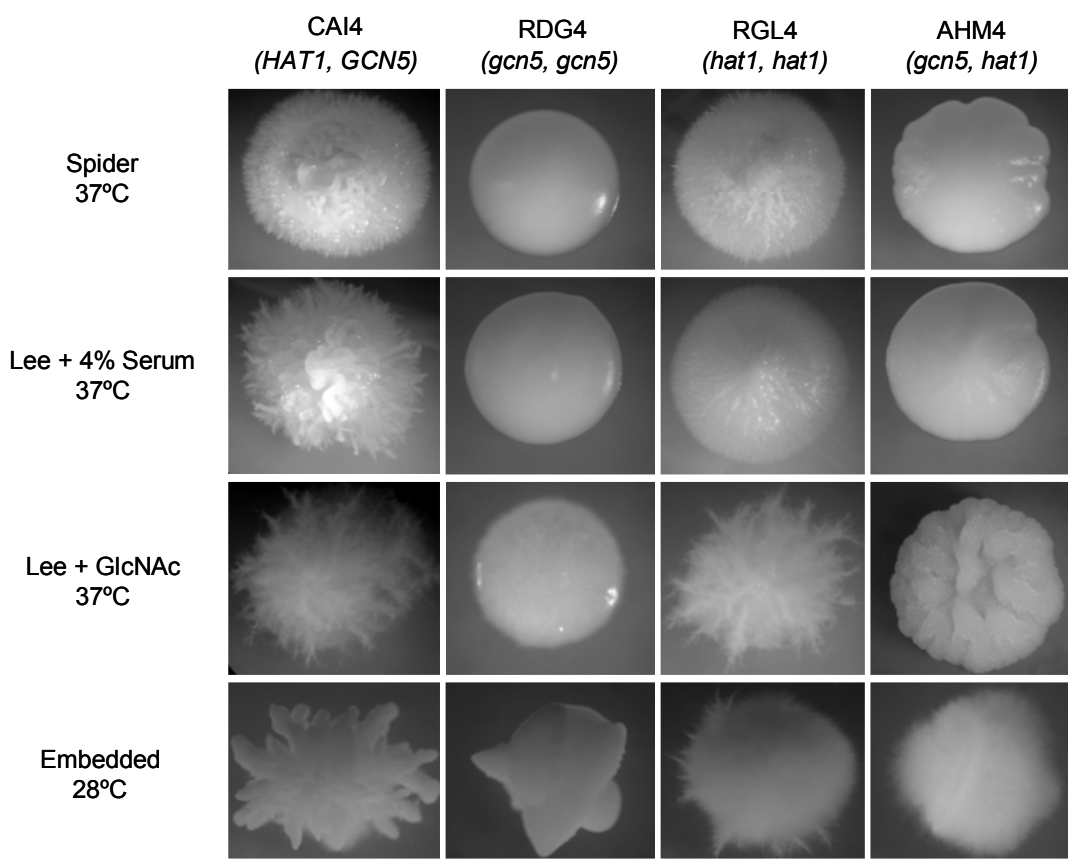

Figure 5.4 Phenotypes of the CAI4, RDG4, RGL4 and AHM4 grown on solid media which induce hyphal development. The plates were incubated for 5 days at $37 \mathrm{C}$.

Cells of the simple and double mutants as well as CAI4 strain were plated at the 50 ufc in solid media plates. Solid media normally induces filamentous growth after 3 days. The CAI4 strain displayed normal filamentous growth, with long chains of 
hypha growing outward from the colony. In contrast, the gcn5, hat1 mutant like the gcn5 single mutant was completely defective for filamentous growth in all media assayed except in embedded agar medium at $28^{\circ} \mathrm{C}$ (Figure 5.4 ); in this medium, the gcn5, hat1 strain showed hyperfilamentation like the hat1 simple mutant.

This fact suggests cooperation of the Hat1p in the suppression of an alternative morphogenetic signalling pathway. It has been reported that deleting the APSES (Asm1, Phd1, Sok2, Efg1 and StuA family) protein genes, EFG1 and EFH1, leads to an increase in filamentation under embedded hypoxic conditions [108]. The present results are very similar to those reported for the efg 1 deletion strain under microaerophilic condition.

\section{Behavior of the C. albicans gcn5, hda1 mutant in liquid media}

The behavior of the double mutant strain was next investigated by assessing hyphal formation in Lee $\left(\mathrm{pH} 6.8,37^{\circ} \mathrm{C}\right)$ or in serum inducing media. For that purpose, C. albicans CAI4, and both simple mutant strains as well as the gcn5, hat1 mutant were grown on different hypha-inducing media. As can be seen in Figure 5.5, the gcn5, hat1 like the both simple mutants failed to form true hypha in the presence of calf serum at $37^{\circ} \mathrm{C}$. The culture was heterogeneous, being composed of a mixture of enlarged spherical cells and short chains of elongated, aberrantly shaped cells attached to one another. This result demonstrated that morphologically gcn5 showed a dominant phenotype in the double mutant cells.

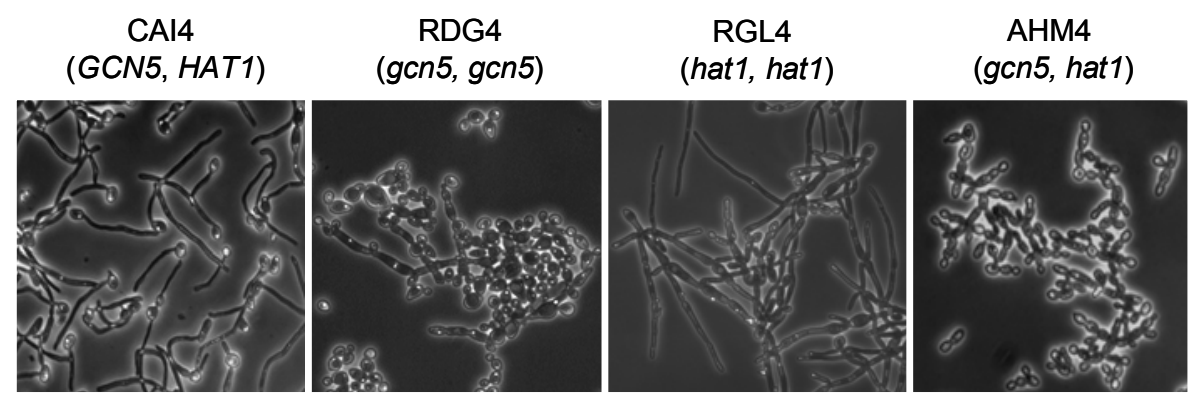

Figure 5.5 Morphology of CAI4, RDG4, RGL4 and AHM4 strains during the yeast-hypha transition in Lee media + $4 \%$ serum at $37^{\circ} \mathrm{C}$

\section{Behavior of the C. albicans gcn5, hda1 in chlamydospore formation}

To examine the association of both the histone acetyltransferase genes with chlamydospore formation, cornmeal agar was used to compare the phenotypes of the CAI4, both simple and the gcn5, hat1 strains. The hat1 simple mutant 
apparently formed chlamydospores like CAI4 strain, whereas the double mutant and the gcn5 simple mutant as showed in Figure 5.6 were defective in the chlamydospore formation.
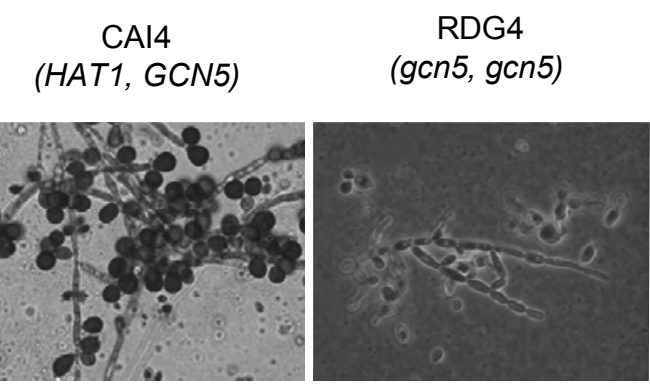

RGL4
(hat1, hat1)

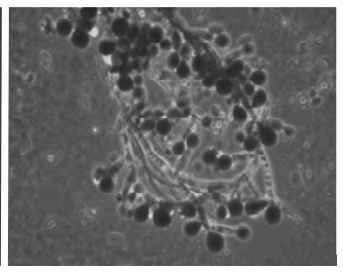

AHM4

(gcn5, hat1)

Figure 5.6 A chlamydospore formation of $C$. albicans is defective in gcn5, hat1 double mutant strain

\section{Virulence}

The observation that the C. albicans GCN5 gene is required for hyphal emergence or elongation in vitro and virulence led to the examination of whether gcn5, hat1 double mutant affects virulence of $C$. albicans. Mice were injected intraperitoneally with a reconstructed URA3 in gcn5, hat1 mutant, and the mouse survival was monitored over a period of 30 days. After three days of infection with $5 \times 10^{8}$ stationary phase cells, mice injected with CAMR (CAI4-URA3 $\left.{ }^{+}\right)$strain showed signs of systemic disease, including weight loss (data not show). Infection with CAMR strain resulted in rapid mortality. No difference in morility was found between mice infected with cells of hat1 [378] and gcn5, hat1 double mutant. Forty percent of mice injected with $5 \times 10^{8}$ cells of the gcn5, hat 1 double mutant survived for the observed period (Figure 5.7). In chapter III, we descrived that $80 \%$ of mice injected with gcn 5 mutant cells survived for same period. This result demonstrated again that hat 1 compensates partially phenotype of the gcn 5 mutation in mice model.

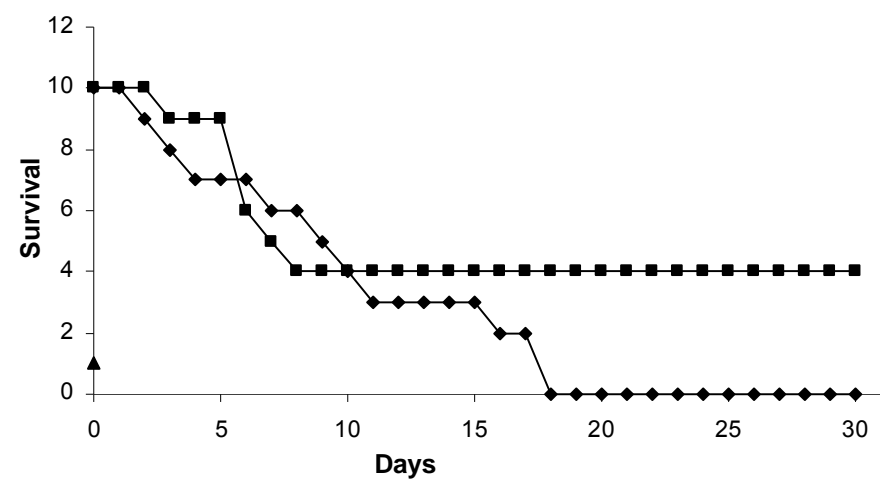

Figure 5.7 Virulence assays. Survival curves for mice $(n=10$ for each $C$. albicans strain at each inoculation dose) infected with $5 \times 10^{8}$ cells of $C$. albicans. CAMR (CAI4-URA+). - HAV1 (gcn5, hat1-URA+). 


\section{Additional phenotypic changes}

The double mutant cells appeared to be more resistant than both hat 1 and gcn5 simple mutants to Zymolyase sensitivity test (Figure 5.8) supporting the idea that Gcn5p and Hat1p jointly control a set of genes involved in the cell wall architecture.

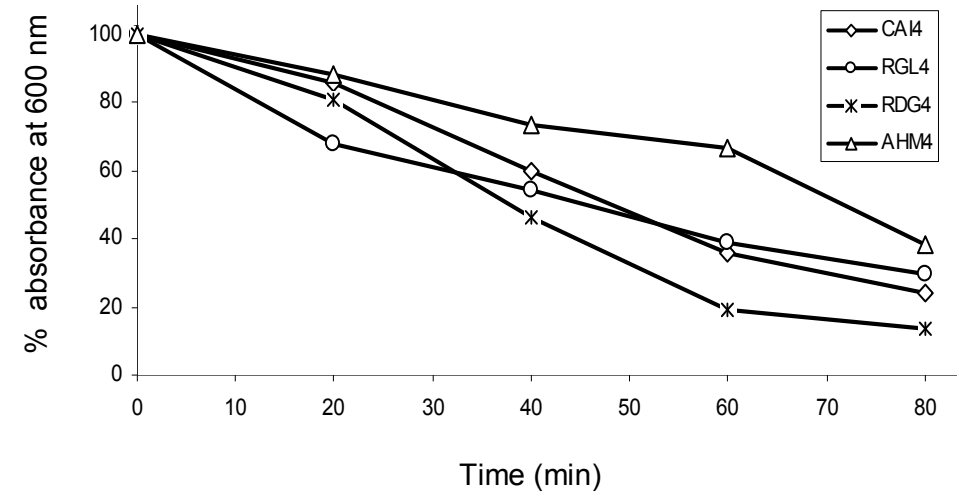

Figure 5.8 Resistance to a cell wall-degrading enzymatic complex of $C$. albicans

The CAI4 $(\diamond)$, RGL4 $(\circ)$, RDG4 $\left(^{*}\right)$ and AHM4 $(\Delta)$ were grown until the exponential phase in YEPD, at $28^{\circ} \mathrm{C}$. Aliquots $(2$ $\times 10^{7}$ cells) were resuspended in $10 \mathrm{mM}$ Tris- $\mathrm{Hcl} \mathrm{pH} 7.5$ and treated with $10 \mu \mathrm{g}$ of Zymolyase per ml. The decrease in optical density (percentage of resistant cells) is monitored every 20 minutes.

Sensitivity to CFW, Congo red, Hygromycin, Itraconazole, Caffeine, Floconazole, $\mathrm{NaCl}$, and SDS

In order to assess the consequences of deletion of GCN5 and HAT1 on the robustness of the cell wall, both single, double mutants, and CAI4 strain were tested for sensitivity to Calcofluor white, $\mathrm{NaCl}$, Congo red and to SDS in plate assays (Figure. 5.9). Deletion of GCN5 increased sensitivity to Calcofluor White, Congo red and SDS, whereas deletion of HAT1 did not (except Congo red). However, the double gcn5, hat1 mutant was more sensitive than both single mutants to Congo red and was also sensitive to Calcofluor white. Deletion of HAT1 gene restored SDS and $\mathrm{NaCl}(1 \mathrm{M})$ tolerance in the gcn5 mutant.

These findings prompted us to carry out a further four phenotypic tests (Hygromycin, Fluconazole, Itraconazole, and Caffeine sensitivity) in order to bether characterize and define the gcn5, hat 1 simple and double mutants. The double mutant like the gcn5 simple mutants was more sensitive to Itraconazole, Fluconazole, Caffeine and Hygromycin than the hat1 mutant and CAI4 strain. 


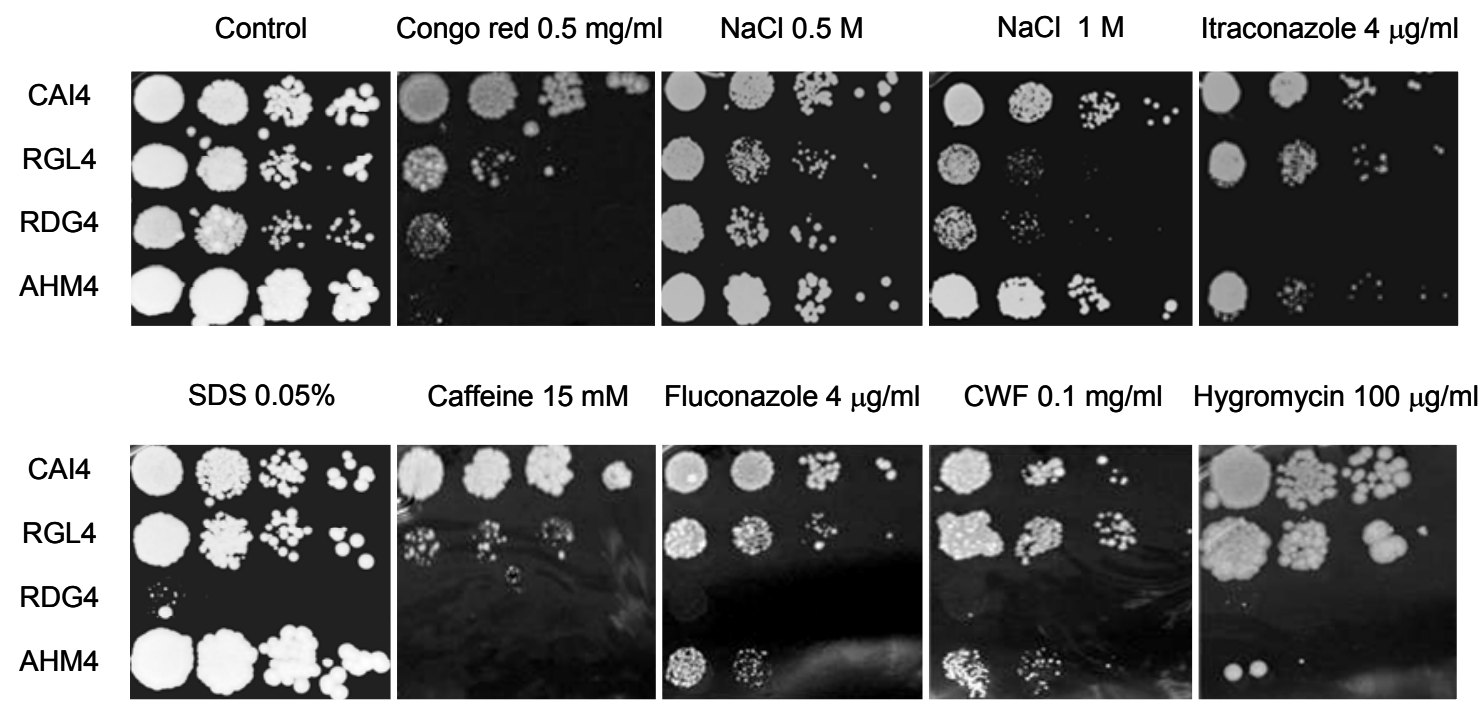

Figure 5.9 Sensibility assays of CAI4, RDG4 (gcn5), RGL4 (hat1) and AHM4 (gcn5, hat1) to different agents. All strains were grown in liquid YEPD. The optical density at $600 \mathrm{~nm}$ of the cultures was adjusted to 0.4 with the same medium and $5 \mu \mathrm{l}$ aliquots from the cultures and from 10 -fold serial dilutions was spotted onto YEPD plates supplemented with different agents. Plates were incubated at $28^{\circ} \mathrm{C}$ for 4 days.

\section{Alcian blue binding assay}

The level of Alcian blue binding for CAI4 was determined both for simple and double mutant strains of $C$. albicans using the method previously described [189]. The gcn5 has been reported to be increased in Alcian blue binding whereas deletion of HAT1 did not. However, the double gcn5, hat1 mutant showed a significant increase in Alcian blue binding with respect to hat1 mutant and CAl4 strain and decreased with respect to gcn5 mutant (Figure 5.10).

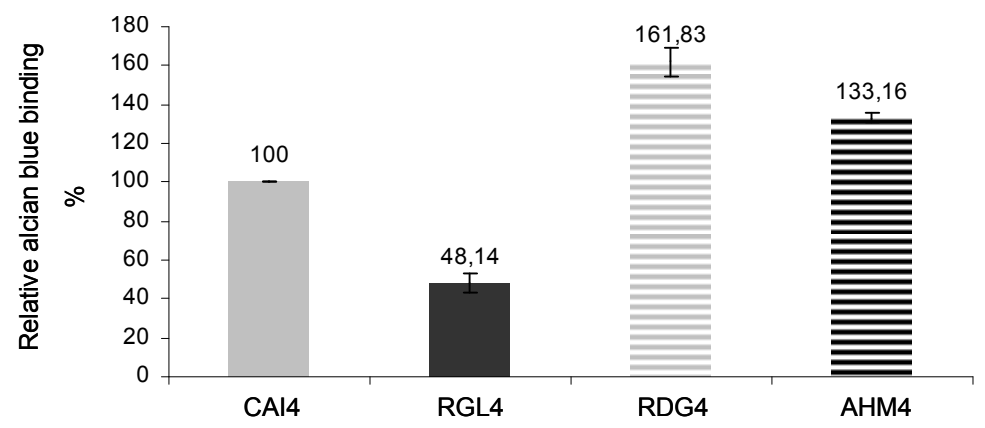

Figure 5.10 Cell surface charge in AHM4 (gcn5, hat1) strain. Alcian blue binding assays. Relative dye binding was calculated as the percentage of dye bound compared with results for the parental strains, CAI4, RDG4 (gcn5) and RGL4 (hat1).

The results suggest that deletion of HAT1 gene slightly compensate phenotype of gcn5 mutant. 


\section{Results of transcription profile}

Transcript profiling was used to further examine the roles of the histone acetyltransferases (HAT1 and GCN5) in C. albicans. cDNA microarrays carrying an almost complete set of $C$. albicans ORFs were used for these experiments, in which the transcriptomes of gcn5, hat 1 mutant were compared with wild-type cells under identical growth conditions. Genes that displayed reproducible and statistically significant changes in expression relative to wild-type cells were identified after exposure to noninducing growth conditions. Lists of all genes that displayed significant regulation by double mutant are provided as supplemental data (Supplementary Tables 5.1 and 5.2).

To explore the functions of the histone acetyltransferases (GCN5 and HAT1) under conditions that promote growth in the yeast form, the transcriptomes of the CAI4 and double mutant strains in YNB medium were compared at $28^{\circ} \mathrm{C}$. The double deletion of GCN5 and HAT1 altered the expression of 521 genes by $\geq 1.5$-fold, with 247 genes being up-regulated and 274 being down-regulated. Only 52 and 283 genes are affected by the deletion of HAT1 and GCN5, respectively. Five genes (FDH3, GRP2, HGT6, HGT8 and IPF3964) were commonly affected in both single and double mutants. The simultaneous deletion of HAT1 and GCN5 showed a transcriptional pattern that overlapped partially with that of gcn5 single mutant (58 genes with 48 genes up- and 10 genes down-regulated). However, a new subset of 453 genes was affected in the gcn5, hat1 double mutant that was not affected in any of single mutants. This result suggested that Gcn5p and Hat1p jointly controlled the regulation of a broad range of genes. Distinct groups of genes were either up- or down-regulated during the yeast mode of growth in the double mutant. Categorization of the regulated genes according to their functional categories of the MIPS database revealed that double deletion of gcn5, hat1 had a striking effect on carbon metabolism (Table 5.3). Transcript levels for almost all glycolytic genes (e.g., PFK1, PFK2 and ENO1) and for genes involved in the metabolism of reserve sugars trehalose and glycogen (e.g., TPS2, TPS3 and GSY1) were down-regulated in the double mutant strain. Transcript levels for some citric acid cycle enzymes (e.g., ACO1 and FUM12), dehydrogenases, glycine (MIS11), and formate catabolic enzymes ( $F D H 1, F D H 3$ and FDH4) were up-regulated (Supplementary Tables 5.1 and 5.2). Almost the same transcriptional profile for glycolytic genes, for genes involved in the metabolism of reserve sugars trehalose and glycogen, acid cycle 
enzymes and formate catabolic enzymes has been reported for efg 1 mutant strain under noninducing conditions [108].

Moreover, Similar to the gcn5 mutant [106], we detected varation of expression several Gcn5p-dependent genes in the double mutant including the up-regulation of genes involved in stress response (ASR1, ASR3, DDR48, FUN12, HSP104, HSP12, HSP31, SSA4 and RTC3), in cell wall proteins (PGA13, PGA56, PIR1, RHD3, YWP1, HPF1 and IPF885), transporter (CFL4, HGT19, PHM7 and PTR21) and encode for transcription factors (NRG1, TBP1 and STP4) and the downregulation two genes encode for ribosomal proteins (RPL10A and RPL20B), two genes encode for cell surface proteins ( $A L S 2$ and $A L S 4)$ and three genes involved in energy pathways (ACS2, MIS11 and TPI1).

Table 5.3 Functional classification of differentially genes during the yeast mode of growth in the gcn5, hat1 doubles mutant cells

\begin{tabular}{|c|c|c|c|}
\hline Gene name & Accession no & Description (CandidaDB) & Fold regulation \\
\hline
\end{tabular}

Down-regulated genes

$\begin{array}{ll}\text { Cell wall } & \\ \text { AAF1 } & \text { orf19.7436 } \\ \text { ALS3 } & \text { orf19.2355 } \\ \text { ALS2 } & \text { orf19.2122 } \\ \text { ALS4 } & \text { orf19.4556 } \\ \text { BGL22 } & \text { orf19.7339 } \\ \text { ECM21 } & \text { orf19.12351 } \\ \text { FGR12 } & \text { orf19.5479 } \\ \text { IFF5 } & \text { orf19.10397 } \\ \text { KRE9 } & \text { orf19.5861 } \\ \text { MNT2 } & \text { orf19.1663 } \\ \text { PGA38 } & \text { orf19.10273 } \\ \text { PGA45 } & \text { orf19.2451 } \\ \text { PGA50 } & \text { orf19.1824 }\end{array}$

$\begin{array}{ll}\text { Adhesion and aggregation mediating surface antigen } & 0.59 \\ \text { Agglutinin like protein } & 0.58 \\ \text { Agglutinin-like protein } & 0.58 \\ \text { Agglutinin-like protein } & 0.54 \\ \text { Glucanase; induced during cell wall regeneration } & 0.61 \\ \text { Involved in cell wall biogenesis } & 0.63 \\ \text { Protein lacking an ortholog in S. cerevisiae; transposon } & 0.60 \\ \text { Putative GPI-anchored protein of unknown function } & 0.62 \\ \text { Cell wall synthesis protein } & 0.50 \\ \text { Alpha-1,2-mannosyl transferase } & 0.26 \\ \text { Putative GPI-anchored protein of unknown function } & 0.57 \\ \text { Cell wall protein; putative GPI-anchor } & 0.55 \\ \text { Putative GPl-anchored protein of unknown function } & 0.62 \\ & \\ \text { Alcohol dehydrogenase } & 0.66 \\ \text { probable alcohol dehydrogenase } & 0.57 \\ \text { Glycerol-3-phosphate dehydrogenase } & 0.54 \\ \text { Glycerol 3-phosphate dehydrogenase } & 0.56 \\ \text { Dihydroorotate dehydrogenase } & 0.18\end{array}$

$\begin{array}{ll}\text { Dehydrogenase } & \\ \text { ADH1 } & \text { orf19.3997 } \\ \text { ADH5 } & \text { orf19.2608 } \\ \text { GPD1 } & \text { orf19.1756 } \\ \text { GPD2 } & \text { orf19.691 } \\ \text { URA1 } & \text { orf19.4836 }\end{array}$

Aerobic Respiration

$\begin{array}{ll}\text { ABC1 } & \text { orf19.10842 } \\ \text { DLD2 } & \text { orf19.6755 } \\ \text { DLD3 } & \text { orf19.5805 } \\ \text { COX8 } & \text { orf19.5213 } \\ \text { COX9 } & 5868 \\ \text { IPF16549 } & \text { orf19.4273 } \\ \text { RIB3 } & \text { orf19.12693 } \\ \text { SHY1 } & \text { orf19.4841 }\end{array}$

ubiquinol--cytochrome-c reductase $\quad 0.57$

D-lactate ferrycytochrome $\mathrm{C}$ oxidoreductase $\quad 0.64$

D-lactate ferrycytochrome $\mathrm{C}$ oxidoreductase $\quad 0.58$

Cytochrome C Oxidase $\quad 0.61$

Cytochrome C Oxidase $\quad 0.64$

Aerobic respiration $\quad 0.57$

3,4-dihydroxy-2-butanone 4-phosphate synthase $\quad 0.53$

$\begin{array}{ll}\text { SURF homologue protein } & 0.44\end{array}$

Secratory pathway

$\begin{array}{ll}\text { BET3 } & \text { orf19.5817 } \\ \text { BET5 } & \text { orf19.302 } \\ \text { Transport } & \\ \text { BPT1 } & \text { orf19.6383 } \\ \text { DUR31 } & \text { orf19.781 }\end{array}$

Targeting and fusion of ER to Golgi transport vesicles 0.62

Targeting and fusion of ER to Golgi transport vesicles $\quad 0.60$ 


$\begin{array}{ll}\text { HAK1 } & \text { orf19.6249 } \\ \text { HGT7 } & \text { orf19.2023 } \\ \text { IFC1 } & \text { orf19.3746 } \\ \text { IFC3 } & \text { orf19.3749 } \\ \text { IPF11077 } & \text { orf19.3432 } \\ \text { NAS2 } & \text { orf19.4550 } \\ \text { OPT1 } & \text { orf19.2602 } \\ \text { ZRT2 } & \text { orf19.1585 }\end{array}$

Potassium transporter 0.56

Hexose transporter $\quad 0.48$

Oligopeptide transporter $\quad 0.44$

Oligopeptide transporter $\quad 0.38$

Membrane transporter 0.58

Membrane transporter $\quad 0.63$

Oligopeptide transporter $\quad 0.10$

Zinc transport protein $\quad 0.37$

Fatty acid metabolism

$\begin{array}{ll}\text { ERG1 } & \text { orf19.406 } \\ \text { ERG251 } & \text { orf19.12101 } \\ \text { ERG9 } & \text { orf19.3616 }\end{array}$

$\begin{array}{ll}\text { Squalene epoxidase } & 0.59\end{array}$

C-4 sterol methyl oxidase $\quad 0.65$

Farnesyl-diphosphate farnesyltransferase $\quad 0.62$

Amino acid metabolism

$\begin{array}{ll}\text { ARO4 } & \text { orf19.4060 } \\ \text { CHA11 } & \text { orf19.7404 } \\ \text { FEN2 } & \text { orf19.12981 } \\ \text { FUR4 } & \text { orf19.313 } \\ \text { HOM3 } & \text { orf19.1235 } \\ \text { MET13 } & \text { orf19.288 } \\ \text { SAM2 } & \text { orf19.657 } \\ \text { Glycolysis } & \\ \text { CDC19 } & \text { orf19.3575 } \\ \text { ENO1 } & \text { orf19.395 } \\ \text { FK26 } & \text { orf19.9844 } \\ \text { PFK1 } & \text { orf19.3967 } \\ \text { PFK2 } & \text { orf19.6540 } \\ \text { PG11 } & \text { orf19.3888 } \\ \text { PGK1 } & \text { orf19.3651 } \\ \text { PGM2 } & \text { orf19.10359 }\end{array}$

3-dehydro-deoxyphosphoheptonate aldolase 0.63

L-serine/L-threonine deaminase $\quad 0.47$

Allantoate permease transporter $\quad 0.61$

Probable uracile or allantoin permease $\quad 0.61$

Aspartokinase $\quad 0.35$

Methylene tetrahydrofolate reductase $\quad 0.47$

S-adenosylmethionine synthetase $2 \quad 0.51$

Pyruvate kinase $\quad 0.40$

Enolase I $\quad 0.63$

6-phosphofructose-2-kinase $\quad 0.44$

6-phosphofructokinase alpha subunit $\quad 0.45$

6-phosphofructokinase beta subunit $\quad 0.45$

Glucose-6-phosphate isomerase $\quad 0.65$

Phosphoglycerate kinase $\quad 0.59$

$\begin{array}{ll}\text { Phosphoglucomutase } & 0.60\end{array}$

Up-regulated genes

$\begin{array}{ll}\text { Oxidative stress } & \text { response } \\ \text { CTA1 } & \text { orf19.6229 } \\ \text { GAD1 } & \text { orf19.1153 } \\ \text { GRP2 } & \text { orf19.4309 } \\ \text { GRP4 } & \text { orf19.10660 } \\ \text { GRP5 } & \text { orf19.6868 } \\ \text { MCR1 } & \text { orf19.3507 } \\ \text { PST2 } & \text { orf19.3612 } \\ \text { TRP99 } & \text { orf19.8054 } \\ \text { TSA1 } & \text { orf19.7417 }\end{array}$

Catalase A. peroxisomal 3.59

Glutamate decarboxylase $\quad 3.18$

Reductase 2.23

Putative reductase $\quad 1.63$

Dihydroflavonol-4-reductases 1.60

$\mathrm{NADH}$-cytochrome-b5 reductase $\quad 1.57$

1,4-benzoquinone reductase $\quad 2.32$

Thioredoxin peroxidase/alkyl hydroperoxide reductase $\quad 1.73$

Protein of TSA/alkyl hydroperoxide peroxidase $\quad 2.26$

Cell wall

$\begin{array}{ll}\text { PGA13 } & \text { orf19.6420 } \\ \text { PGA39 } & \text { orf19.6302 } \\ \text { PGA4 } & \text { orf19.4035 } \\ \text { PGA56 } & \text { orf19.1105.2 } \\ \text { PGA6 } & \text { orf19.12229 } \\ \text { PIR1 } & \text { orf19.220 } \\ \text { RBT5 } & \text { orf19.5636 } \\ \text { RHD3 } & \text { orf19.5305 } \\ \text { TOS1 } & \text { orf19.1690 } \\ \text { YWP1 } & \text { orf19.3618 }\end{array}$

Putative GPI-anchored protein $\quad 2.39$

Putative GPI-anchored protein of unknown function $\quad 2.15$

Putative GPI-anchhored protein $\quad 1.63$

Putative GPI-anchor 2.53

Putative GPI-anchored cell-wall protein $\quad 2.02$

Putative cell wall protein of the PIR family 2.39

GPI-anchored cell wall protein involved in hemoglobin utilization $\quad 1.69$

GPI-anchored protein that localizes to the cell wall $\quad 1.57$

Putative Anchor subunit of a-agglutinin $\quad 2.20$

$\begin{array}{ll}\text { Putative cell wall protein } & 1.56\end{array}$

Oxidase/Peroxidase

$\begin{array}{ll}\text { CFL1 } & \text { orf19.1263 } \\ \text { CFL4 } & \text { orf19.9488 } \\ \text { CFL5 } & \text { orf19.1930 } \\ \text { IPF6231 } & \text { orf19.10802 } \\ \text { IPF3358 } & \text { orf19.4016 } \\ \text { IFG3 } & \text { orf19.944 } \\ \text { PUT1 } & \text { orf19.4274 } \\ \text { TRP99 } & \text { orf19.8054 } \\ \text { TSA1 } & \text { orf19.7417 }\end{array}$

Ferric reductase 1.69

Ferric reductase $\quad 1.72$

Ferric reductase $\quad 1.95$

Prolin-methionine-R-oxid reductase $\quad 1.55$

Ubiquinol-cytochrome-c reductase $\quad 1.63$

Probable d-amino acid oxidase $\quad 1.90$

$\begin{array}{ll}\text { Proline oxidase } & 1.57\end{array}$

Protein described as thioredoxin peroxidase 1.73

Protein of TSA/alkyl hydroperoxide peroxidise 2.26

Dehydrogenase

Putative aldehyde dehydrogenase $\quad 1.68$ 


\begin{tabular}{|c|c|c|c|}
\hline $\mathrm{CSH} 1$ & orf19.4477 & Putative aryl-alcohol dehydrogenase & 2.82 \\
\hline EBP5 & orf19.3234 & NADPH dehydrogenase & 1.62 \\
\hline $\mathrm{FDH} 1$ & orf19.1774 & Putative formate dehydrogenase & 1.53 \\
\hline FDH3 & orf19.7600 & Glutathione-dependent formaldehyde dehydrogenase & 1.80 \\
\hline FDH4 & orf19.1774 & Formate dehydrogenase & 2.25 \\
\hline IFD1 & orf19.8650 & Putative aryl-alcohol dehydrogenase & 1.93 \\
\hline IFD6 & orf19.1048 & Putative aryl-alcohol dehydrogenase & 1.87 \\
\hline IPF5389 & orf19.5565 & 3-hydroxyisobutyrate dehydrogenase & 1.59 \\
\hline LPG20 & orf19.771 & Putative oxidoreductase & 1.71 \\
\hline PUT2 & orf19.3974 & 1-pyrroline-5-carboxylate dehydrogenase & 2.69 \\
\hline \multicolumn{4}{|c|}{ Transporter } \\
\hline ACR1 & orf19.3931 & Succinate-fumarate transporter & 1.93 \\
\hline CCC1 & orf19.6948 & Transmembrane $\mathrm{Ca}^{2+}$ transporter & 2.13 \\
\hline HGT11 & orf19.4527 & Hexose transporter & 3.37 \\
\hline HGT12 & orf19.3668 & Hexose transporter & 3.44 \\
\hline HGT17 & orf19.4682 & Putative glucose transporter & 1.69 \\
\hline HGT19 & orf19.5447 & Putative glucose/myo-inositol transporter & 2.37 \\
\hline HGT6 & orf19.2020 & Sugar transporter & 1.52 \\
\hline HGT8 & orf19.2021 & Sugar transporter & 1.57 \\
\hline HIP1 & orf19.4940 & Histidine permease & 1.62 \\
\hline JEN2 & orf19.12767 & Carboxylic acid transporter protein & 3.02 \\
\hline PTR21 & orf19.6937 & Peptide transporter & 1.86 \\
\hline SGE11 & orf19.4779 & Putative transporter & 2.26 \\
\hline STL1 & orf19.13176 & Sugar transporter & 2.01 \\
\hline
\end{tabular}

We have detected regulation genes encoding, for cell wal proteins including the upregulation PGA39, PGA4, PGA56, PGA6, RBT5, TOS1 and down-regulation ALS3, ALS2, BGL22, ECM21, FGR12, IFF5, KRE9, MNT2, PGA38, PGA45 and PGA50 in the double mutant during the yeast mode of growth. We detected the up-regulation many genes involved in oxidative stress response (CTA1, GAD1, GRP2, GRP4, GRP5, MCR1 and PST2), transporters (ACR1, CCC1, JEN2, HIP1, PTR21 and SGE11), ferric reductases (CFL1 and CFL5), oxidases/perxodases (PF6231, PF3358, IFG3, PUT1, TRP99 and TSA1), dehydrogenases (ALD5, ALD6, EBP5, IFD1, IPF5389 and PUT2). Among the up-regulated genes, four genes (HGT11, HGT12, HGT17 and STL1) were related to glucose transport. This result is in strong contrast to those reported in the gcn5 and hat1 simple mutant [106], in which these groups of genes are not regulated during yeast mode of growth. Further groups of genes regulated jointly by Gcn5p and Hat1p include genes involved in components of the respiratory chain (ABC1, DLD2, DLD3, COX8, COX9, IPF16549, RIB3 and SHY1), biosythesis of ergoestrol (ERG1, ERG251 and ERG9), amino acid metabolism (ARO4, CHA11, FEN2, FUR4, HOM3 and MET13) and genes encoding for transporters (BPT1, DUR31, HAK1, FC1, IFC3, IPF11077, NAS2 and ZRT2). 


\section{Discussion}

Previous analysis has shown that members of the histone acetyltransferase family (GCN5 and HAT1) are required for hypha formation in C. albicans in liquid and solid media. The present phenotypic analysis indicates that growth of the gcn5, hat1 double mutant is associated with a detectable deficiency in the rate of colony formation on rich mediua plates at $28^{\circ} \mathrm{C}$. Morphologically, the gcn5, hat 1 double mutant and both simple mutant strains have shown a phenotype very different to CAI4 strain. Thus, under our experimental conditions, Gcn5p and Hat1p appear as important proteins for the regulation of the genes that are critical for cell growth and morphology. The most prevalent phenotype is the inability of the gcn5, hat1 strain to switch from the yeast to filamentous form in liquid media containing serum at $37^{\circ} \mathrm{C}$ and in all the solid media assayed except in embedded medium. In embedded medium, the gcn5, hat1 like the hat1 strain was hyperfilamentous when compared to the wild type. In contrast, the gcn5 simple mutant strain has failed to form filamentation in embedded medium in agreement with those reported previously by Doedt et al [108]. Other phenotypes observed include sensitivity of the double mutant like gcn5 simple mutant to Congo red, Calcofluor white, and Caffeine suggesting a remodelling of cell wall composition in the double mutant. No difference in sensitivity was found towards SDS and $\mathrm{NaCl}$ when compared to CAI4 strain (Figure 5.9) suggesting that deletion of HAT1 gene restored SDS and $\mathrm{NaCl}$ (1 M) tolerance in the gcn5 mutant.

The study of chlamydospore formation has been performed to determine whether members of histone acetyltransferases that block hyphal development also have a function in the formation of chlamydospores. The gcn5, hat1 strain like gcn5 mutant strain is defective for chlamydospore formation. However, similar to CAI4 strain, hat1 mutant has competently formed chlamydospores [106]. These results demonstrate that Gcn5p like Efg1p is needed for chlamydospore formation, and that Gcn5p produces the predominant phenotypes in double mutant cells.

Upon comparing our results and those reported in the both gcn5 and hat 1 simple mutant [106], we observed that expression several of genes involved in the glucolytic patway, oxidative estress, amino acide metabolism, glocuse transport, genes encoding for cell wall proteins were regulate jointly by Gcn5p and Hat $1 p$. We detected expression 58 genes were regulated commonly in the double mutan and gcn5 simple mutant. Of those, genes involved in stress response, energy 
pathways, encoding for cell wall proteins, transcription factors and transportes were the predominant ones. There was, however, little overlap between the regulated genes reported in these studies and those presented in the hat1 mutant [106] that is consistent with dominant phenotype of gcn5 simple mutant.

Down-regulation of a number of genes involved in ergosterol biosynthesis has been observed. Ergosterol genes were mainly upregulated after exposition to antifungal agents $[231,281]$. This result is consistent with the results showing that the double mutant grown as yeast exhibited azole sensitivity. 


\section{Introducción}

El reino de los hongos incluye numerosas especies importantes desde diversos puntos de vista como son el ecológico, el económico o el médico. Las especies fúngicas al descomponer la materia orgánica contribuyen a la continuidad del ciclo de nutrientes dentro de los ecosistemas, la mayoría de plantas vasculares no podrían crecer sin los hongos simbióticos asociados a sus raíces. Algunos hongos proporcionan de forma directa alimento al hombre y otros son utilizados para dar determinadas características a productos consumidos por el hombre, como el pan. Resulta de especial relevancia médica la capacidad de algunas especies de este reino de sintetizar y secretar al medio antibióticos. Por otro lado, algunas especies fúngicas causan infecciones en animales y plantas que resultan difíciles de tratar, debido a que las células fúngicas son células eucariotas que realizan sus procesos metabólicos de forma similar a las células de organismos superiores. Los hongos son organismos ubicuos encontrándose especies fúngicas en casi todos los hábitats. Muchas de estas especies son capaces de adaptarse a diferentes ambientes, desarrollando en un corto periodo de tiempo una respuesta transcripcional y fisiológica determinada ante un cambio de las condiciones del medio [451].

\section{Candida albicans}

\section{Características generales. Posición taxonómica}

El género Candida engloba unas doscientas especies que comparten características como reproducción asexual por gemación y ausencia de pigmentos carotenoides. Las especies del género Candida se incluyeron inicialmente dentro de la clase Deuteromycetes, grupo que incluye los comúnmente llamados "hongos imperfectos" y que corresponden con especies no relacionadas cuya principal característica es la ausencia de ciclo sexual conocido. Actualmente el género Candida se ha incluido dentro de los ascomicetos de acuerdo con la siguiente clasificación [51]:

Reino: Fungi

Phyllum: Ascomycota

Clase: Hemiascomycetes

Orden: Saccharomycetales 
Familia: Candidaceae

Género: Candida

El cambio de clasificación se ha debido a varios factores. Datos de comparación de secuencias de diferentes genes indican que filogenéticamente las especies con las que están más relacionadas las especies del género Candida son ascomicetos. Además, todas las especies de Candida presentan características que comparten con los ascomicetos y las diferencian de los basidiomicetos, como es la gemación de una yema por locus, en lugar de la gemación sucesiva de varias yemas a partir de un mismo locus, propia de los basidiomicetos [51]. C. albicans es la especie más estudiada de su género ya que es el patógeno fúngico oportunista de mayor incidencia en humanos. Los rasgos más significativos de $C$. albicans son su capacidad de formar tubos germinativos y clamidosporas, seguida de su patrón de asimilación de azúcares. Se han establecido varios biotipos dentro de la especie basándose en similitudes fenotípicas y de DNA [358].

\section{El genoma de C. albicans}

C. albicans es un organismo diploide. Desde el año 2000 se dispone de la secuencia completa de su genoma. La secuenciación fue llevada a cabo por el Stanford Genome Center partiendo de DNA de la cepa SC5314. Dicha estirpe fue elegida por ser un aislado clínico usado habitualmente en análisis moleculares y ensayos de virulencia, y poseer un cariotipo estándar. Se ha estimado que el genoma haploide de $C$. albicans comprende aproximadamente $16 \mathrm{Mb}$, siendo por tanto un $30 \%$ mayor que el de $S$. cerevisiae. Se han realizado sucesivos ensamblajes de las secuencias que han sido publicados en la página web http://www sequence.stanford.edu/group/candida/download.html. Recientemente se ha publicado el ensamblaje 20 , en el que se han podido anillar casi en su totalidad las secuencias de los supercontigs del ensamblaje 19 en los 8 cromosomas de $C$. albicans. Se ha realizado un mapa físico de todos los ORFs que se encuentra disponible en la página http://albicansmap.ahc.umn.edu/ (University of Minnesota y Biotechnology Research Institute de Montreal). El DNA mitocondrial de C. albicans ha sido también secuenciado, estimándose un tamaño total de 40,420 pares de bases (Número de acceso GeneBank: AF285261). A partir del ensamblaje 19 varias organizaciones llevaron a cabo una anotación de las posibles secuencias de marcos de lectura abierta (ORFs) presentes en el genoma de C. albicans. Así, por 
primera vez se hizo pública la base de datos CandidaDB (http://www.pasteur.fr Galar Fungail/CandidaDB) creada por el consorcio europeo Galar Fungail Group, del cual forma parte nuestro grupo de investigación. Se consideraron ORFs relevantes para la anotación aquellas que codificaran una secuencia de más de 150 aa, que tuvieran un homólogo en las bases de datos públicas o tuvieran una probabilidad significativa de codificar una proteína según la predicción del programa GeneMark. Se identificaron 6244 ORFs, 130 de ellas codifican tRNAs. Se les dió nombre y función a aquellos ORFs que correspondían con genes previamente caracterizados o que por su elevado porcentaje de identidad con un gen de $S$. cerevisiae probablemente codificaran su homólogo funcional en C. albicans.

\section{Características de las diferentes morfologías}

C. albicans es un organismo polimórfico, dada su capacidad de crecer en forma de hifa, levadura, pseudohifa o dar lugar a la formación de clamidosporas. Las levaduras o blastosporas tienen forma elipsoidal con un diámetro aproximado de 5 $\mu \mathrm{m}$, se dividen por gemación y son más abundantes durante el crecimiento saprófito del hongo [358]. Las hifas tienen forma cilíndrica con un diámetro aproximado de $2 \mu \mathrm{m}$, las paredes laterales se mantienen paralelas a lo largo de todo el filamento y no presentan constricciones en los septos [451]. Las pseudohifas consisten en cadenas de células alargadas que se originan mediante un proceso de gemación en el que no se llegan a separar la célula madre y la célula hija. A diferencia de las hifas, las paredes laterales de las pseudohifas no se mantienen paralelas, por lo que muestran valores de diámetro variable, son más gruesas (el diámetro mínimo se encuentra alrededor de $2.8 \mu \mathrm{m}$ ) y presentan constricciones en los septos [358,451]. Las clamidosporas son esporas asexuales con una pared gruesa que se forma mediante el recubrimiento de las células preexistentes, son redondeadas con un tamaño aproximado de 8 a $12 \mu \mathrm{m}$ de diámetro y aparecen en posiciones laterales o terminales de hifas y pseudohifas, sobre células suspensoras alargadas. In vivo rara vez se detectan estas estructuras [70]. En el laboratorio (in vitro) su formación se induce en medios sólidos pobres en nutrientes, suplementados con detergentes, en condiciones microaerófilas y a temperaturas entre $20^{\circ} \mathrm{C}$ y $30^{\circ} \mathrm{C}$, mientras que su formación se reprime en presencia de glucosa [51,353]. La capacidad de formar clamidosporas es una característica casi exclusiva de $C$. albicans solo compartida por $C$. dubliniensis y 
algunas cepas de $C$. tropicalis $[51,440]$. En ocasiones las hifas y las pseudohifas son estructuras similares que resultan difíciles de diferenciar. Una forma clara de determinar si se trata de una u otra morfología consiste en visualizar el primer septo formado entre la levadura madre y el filamento. En el caso de las hifas este primer septo se forma dentro del tubo germinativo, mientras que en las pseudohifas se forma en el cuello de la célula madre [156,497]. Esta diferencia se debe a que en las hifas la elongación del tubo germinativo ocurre antes del inicio del ciclo celular, marcado por la síntesis de DNA, mientras que en las levaduras y pseudohifas la gemación depende del inicio del ciclo celular [182,451]. Las células de C. albicans también pueden dar lugar a la formación de biopelículas, se trata de comunidades de células organizadas en estrecha relación con la superficie sobre la cual se forman. En el caso de C. albicans, los biopelículas están constituidos por levaduras, hifas, pseudohifas y material extracelular (polisacáridos y proteínas) que se disponen de forma ordenada en capas. Generalmente las capas internas están constituidas mayoritariamente por levaduras mientras que en las más externas se encuentran principalmente formas filamentosas. El estudio de los biopelículas de $C$. albicans posee gran interés médico dada su aparición en epitelios, catéteres y prótesis y su marcada resistencia a antifúngicos [109,139].

\section{Factores de virulencia de $C$. albicans}

El espectro de afecciones causadas por $C$. albicans es superior al de la mayoría de patógenos oportunistas, lo que es atribuible a su capacidad para sobrevivir en diferentes tejidos del hospedador; cada uno de ellos asociado a un conjunto diferente de presiones ambientales, y a un amplio repertorio de factores de virulencia [53]. Se conoce poco acerca de la biología de C. albicans como comensal, tan solo se sabe que pequeñas variaciones en el sistema inmune del hospedador determinan la forma de crecimiento, por lo que la línea que separa la forma comensal de la patógena parece ser realmente delgada. El método en el que se han basado la mayor parte de los investigadores para considerar una determinada propiedad como factor de virulencia consiste en demostrar que las cepas carentes de dicha actividad no son capaces de establecer infecciones, pero si son capaces de vivir como comensales. Las etapas sucesivas del proceso de infección y los diferentes tipos de patologías causados por $C$. albicans están condicionadas por diferentes combinaciones de estos factores de virulencia [337]. 
Entre los factores de virulencia atribuidos tradicionalmente a $C$. albicans se encuentran la presencia en la superficie celular de proteínas de adhesión a tejidos del hospedador, la secreción de enzimas líticas y la capacidad de llevar a cabo la transición dimórfica [81]. El denominado cambio fenotípico espontáneo o "switching", la capacidad de asimilación de hierro a partir de tejidos del hospedador y propiedades como el tigmotropismo y el mimetismo molecular constituyen otros posibles factores de virulencia de C. albicans aún poco caracterizados $[53,135,337,498]$.

\section{Mecanismos de regulación transcripcional}

\section{Características generales}

La regulación de la expresión génica se realiza a diferentes niveles que se encuentran relacionados entre sí. En los promotores de todos los genes se encuentran secuencias reguladoras comunes que son reconocidas por la maquinaria de la RNA polimerasa II, tales como las secuencias situadas en el extremo 5' denominadas "uppstream activating sequences" (UAS) y las secuencias TATA. Además, es necesaria para la transcripción la presencia del elemento iniciador, que determina el punto donde comienza la transcripción y que se encuentra siempre en el extremo 3' de la caja TATA, pero para el cual no se ha establecido una secuencia consenso. En determinados promotores se encuentran sitios de reconocimiento para factores de transcripción específicos, que activan o reprimen la transcripción en respuesta a diferentes estímulos. Uno de los mecanismos de regulación de la transcripción consiste en la modulación de la actividad y concentración de las proteínas que actúan como activadores y represores de la transcripción, produciéndose como consecuencia la expresión diferencial de los genes diana de estos reguladores. Muchos activadores actúan sobre las histonas y el DNA provocando fenómenos de reposicionamiento o desensamblaje de los nucleosomas, que afectan a la accesibilidad de la maquinaria de transcripción general.

\section{La cromatina}

El DNA es la molécula biológica que contiene la información genética de los organismos, y en las células eucariotas se aloja en el núcleo. Dadas las dimensiones del DNA, que es una molécula muy larga, es necesario plegarlo 
previamente para almacenarlo en el espacio limitado del núcleo. Para ello las moléculas de DNA se asocian con diferentes proteínas compactándose en una estructura organizada y jerárquica denominada cromatina [176,503]. Esta es una estructura inherentemente represiva que dificulta los procesos en los que se requiere acceder a la secuencia de DNA. Las proteínas mayoritarias de la cromatina son las histonas, unas proteínas de baja masa molecular, ricas en aminoácidos básicos. Las histonas están muy conservadas en todos los organismos eucariotas [465], lo que sugiere que la estructura fundamental de la cromatina debió aparecer en un antecesor común, posiblemente con el objetivo de resolver el problema del empaquetamiento del DNA dentro del núcleo [429]. La cromatina no es una estructura estática y cerrada en la que histonas y otras proteínas se asocian al DNA con una función únicamente estructural, sino que estas proteínas son sometidas a diversos tipos de modificaciones y alteraciones, lo que se traduce en una relajación de la estructura de la cromatina. Así pues, la cromatina es una estructura dinámica que puede adoptar diferentes estados de compactación regulando el acceso a la información contenida en el DNA, y permitiendo la ejecución de procesos celulares pre-programados como son el ciclo celular, la diferenciación celular o la adecuación de la respuesta transcripcional para la adaptación a situaciones ambientales cambiantes. Procesos fisiológicos como la replicación, la transcripción o la reparación del DNA, también precisan que se pueda regular el grado de compactación de la cromatina. Las histonas internas son proteínas muy conservadas, pequeñas y ricas en lisinas y argininas, lo que les confiere un carácter fuertemente básico. Las masas moleculares de las histonas de S. cerevisiae son: H2A 14.28 kDa; H2B 14.24 kDa; H3 15.37 kDa y H4 11.37 $\mathrm{kDa}$. Desde el punto de vista estructural, las histonas tienen dos dominios: el dominio globular de la región C-terminal y un dominio no estructurado de la región $\mathrm{N}$-terminal. El dominio globular contiene el motivo 'histone fold', formado por unos 65 aminoácidos que se estructuran en una hélice a corta seguida de un giro/hoja $\beta$, una hélice a larga, otro giro/hoja $\beta$, y por último otra hélice $\alpha$ corta. Las tres hélices del dominio 'histone fold' de una histona encajan con las tres hélices del dominio 'histone fold' de otra histona. Este dominio participa en interacciones histona-histona y también en interacciones histona-DNA en el nucleosoma. Los extremos $\mathrm{N}$-terminales también participan en la interacción con nucleosomas adyacentes [291]. Algunos de los residuos de las histonas son susceptibles de 
sufrir modificaciones postraduccionales, tales como acetilación, metilación, fosforilación, ubiquitinación y sumoilación. Aunque generalmente se ha aceptado que estas modificaciones afectan principalmente a residuos localizados en los dominios $\mathrm{N}$-terminales, recientemente se han identificado residuos de los dominios C-terminales que también sufren modificaciones postraduccionales [531]. Todas estas modificaciones participan en la regulación de la actividad de la cromatina. Además de las histonas internas, la cromatina también contiene la histona $\mathrm{H} 1$, que se dispone en el exterior de los nucleosomas. Existen diferentes variantes de la histona $\mathrm{H} 1$ que constituyen una compleja familia de proteínas y que pueden ser específicas de tejido o del momento del desarrollo [234]. Las variantes de $\mathrm{H} 1$ son parcialmente redundantes entre sí y pueden regular la actividad de la cromatina estabilizando la fibra de $30 \mathrm{~nm}$ y la posición de los nucleosomas, o alterando la accesibilidad de factores reguladores [49,136]. Además de todos estos participantes, también interviene una amplia variedad de proteínas no histona, poliaminas e iones metálicos divalentes en el establecimiento y regulación de la estructura de la cromatina.

\section{Regulación de la actividad de la cromatina}

Como se ha descrito anteriormente, la cromatina esta formada por la asociación de proteínas al DNA, lo que da lugar a una estructura de naturaleza represiva que inhibe procesos como la transcripción, la reparación y la replicación del DNA. Sin embargo la cromatina no es una estructura estática, sino variable y dinámica, que participa en la regulación de todos estos procesos. Existen diferentes tipos de complejos proteicos que modulan la estructura de la cromatina: complejos que modifican histonas químicamente, complejos que remodelan cromatina mediante el empleo de la energía del ATP, y complejos que modifican el DNA. Además la constitución de nucleosomas con variantes de histonas, diferentes de las histonas canónicas, confiere diferentes propiedades estructurales a la cromatina.

\section{Modificaciones postraduccionales de las histonas}

Las primeras modificaciones postraduccionales identificadas sobre las histonas se localizaron en residuos de los extremos $\mathrm{N}$-terminales. Se ha asignado, por ello, una especial atención a estas regiones, relacionándolas con la regulación de la actividad de la cromatina. Sin embargo, recientemente se han identificado diversas 
modificaciones postraduccionales sobre residuos localizados en la región globular de las histonas [136,531]. La acetilación es la modificación postraduccional de histonas más estudiada, ya que es la que se conoce desde hace más tiempo. La acetilación de lisinas neutraliza la carga positiva del aminoácido y desde su descubrimiento se ha pensado que esto podría disminuir la fuerza de su interacción con el DNA, afectando así a la estructura de la cromatina. La acetilación está catalizada por enzimas histona acetiltransferasa (HAT) y puede ser eliminada por histona desacetilasas (HDAC).

\section{Acetilación}

La acetilación de lisinas es la modificación postraduccional de histonas de la que se han realizado un mayor número de estudios en una gran diversidad de organismos eucariotas, desde la levadura hasta plantas y humanos. Desde siempre, se ha pensado que cuando las lisinas no están acetiladas y por tanto tienen una carga positiva, se favorece la interacción de los extremos $\mathrm{N}$-terminales con el DNA generándose una cromatina más compacta y reprimida. La acetilación de lisinas, al eliminar la carga positiva, debilitaría la interacción de los extremos $\mathrm{N}$ terminales con el DNA provocando la relajación de la estructura de la cromatina. Por tanto, aunque no siempre es cierto, generalmente se ha relacionado la acetilación de histonas con la expresión génica, de manera que las histonas hiperacetiladas se encuentran asociadas con regiones genómicas transcripcionalmente activas, mientras que la desacetilación de histonas da lugar a la represión y al silenciamiento [252,474]. Sin embargo, actualmente se sabe que la acetilación de histonas está relacionada con muchos otros procesos fisiológicos, además de la regulación de la transcripción, como la progresión del ciclo celular, recombinación y reparación del DNA, apoptosis, elongación de la transcripción o el ensamblaje de nucleosomas [252,304].

\section{Histona acetiltransferasas}

En la cromatina de los organismos eucariotas el DNA se organiza alrededor de octámeros de histonas, dando lugar a la estructura nucleosomal. Las histonas sufren una serie de modificaciones postraduccionales entre las que se encuentra la acetilación de residuos de lisina, situados en los extremos $\mathrm{N}$-terminales de estas proteínas. Esta modificación reversible, catalizada in vivo por las histona 
desacetilasas (HD) y las histona acetiltransferasas (HAT), ha sido implicada durante muchos años en la regulación de diversos procesos celulares de gran importancia. Recientemente, el papel de la acetilación de histonas en la regulación de la transcripción ha cobrado gran importancia con el descubrimiento de que proteínas con actividad HAT y HD son reguladores transcripcionales.

En la levadura Saccharomyces cerevisiae se han identificado genes que codifican para cuatro proteínas con actividad acetiltransferasa, GCN5, HAT1, ESA1 y TAF130 que ya se habían detectado previamente por métodos bioquímicos

\section{La acetiltransferasa de histona de C. albicans GCN5}

El coactivador transcripcional de levadura GCN5 forma parte de un gran complejo multimérico que es requerido para el remodelamiento de la cromatina y la activación transcripcional. GCN5 está conservado en las especies de Candida y en la mayoría de las regiones homólogas forman parte del dominio HAT y el bromodominio (Figura 1.7 y 1.8). Muchos genes de levadura codifican proteínas acetiltransferasas de histonas y desacetilasas de histonas en C. albicans y su fenotipo relativo es totalmente desconocido. En nuestro laboratorio se han diseñado un análisis fenotípicos de cepas de levadura que contienen deleciones en GCN5 y otros genes que codifican para miembros de las familias HATs y HDACs de $C$. albicans. El genoma de $C$. albicans contiene seis ORFs que potencialmente codifican proteínas desacetilasas de histonas conteniendo el dominio HDACs y cinco acetiltransferasas de histonas. Una búsqueda en la base de datos de la NCBI (http://www.ncbi.n/m.nih.gov/BLAST/) indica que una proteína de Candida albicans llamada Gcn5 (orf19.705) comparte la mayor similitud con Pichia guilliermondii ATCC 6260 (81\%), Pichia stipitis (78\%), Lodderomyces elongisporus (70\%), Debaryomyces hansenii (73\%) y Saccharomyces cerevisiae (70\%) (Figura 1.8). El número de acceso en el GeneBank para la secuencia nucleotídica de la GCN5 de C. albicans es NW_139602.

La proteína Gcn5p de levadura, ancestro de la familia de las acetiltransferasas de histona (HAT), transfieren un grupo acetilo desde el acetil-coenzima $A$ al grupo $\varepsilon$ amino de la cadena lateral de residuos de lisina específicos de histonas H3 y H4 [392,516]. Gcn5p no actúa por cuenta propia, sino como la subunidad catalítica de dos complejos separados y altamente conservados llamados ADA y SAGA (SptAda-Gcn5-acetiltransferasa). Las proteínas Spt se encuentran exclusivamente en 
el complejo SAGA, Spt20p es necesaria para el mantenimiento de la integridad y la función del complejo entero [517]. La acetilación de histonas por la proteína Gcn5p está implicada en el desplazamiento de los nucleosomas de los promotores durante la activación transcripcional y también en ayudar al reclutamiento de la proteína de unión TATA, la RNA polimerasa II y coactivadores [154]. La acetilación por lo tanto facilita la formación de una estructura de cromatina "abierta" correspondiente al genoma transcribiendose [99,251,449]. Durante la división celular, la remodelación de la cromatina se extiende a lo ancho de las regiones cromosomales, produciendo grandes longitudes de compactación y recondensación a lo largo de todo el genoma en cada división celular [93,489]. Perturbar el equilibrio entre la HAT y la desacetilasa de histona altera las actividades de las proteínas a una escala celular, lo cual conduce a varias enfermedades, incluyendo el cáncer. Varios trabajos han resaltado la participación de Gcn5p sola o en combinación con otras HATs tales como Sas3p en el ciclo celular [199,223] y en la regulación transcripcional de un grupo de genes requeridos al final de la telofase [247]. En células de mamíferos, la pérdida del homólogo Gcn512p es letal durante la embriogénesis, induce un alto nivel de apoptosis [519], y afecta la transición G2/M en mutantes nulas de células madre en embriones de ratón [277]. En levaduras, el nucleosoma centromérico especializado, el cual contiene la proteína Cse4p variante de la histona H3 [318], es necesaria para el ensamblaje y las interacciones de los componentes internos del cinteocoro en el centrómero [72] y para la correcta fijación de los cromosomas al huso en la metafase. Mutaciones en los componentes del centrómero/cinetocoro o modificaciones epigenéticas de esta estructura pueden dar lugar a una incorrecta segregación del cromosoma y en el retraso de G2/M. En la levadura la Gcn5p está físicamente relacionada con el centrómero. Se ha descrito regulación epigenética dependiente de Gcn5p en el centrómero/cinetocoro en mitosis [488].

\section{Histona desacetilasas}

Las histona desacetilasas (HDACs) son enzimas hidrolíticas capaces de revertir la acetilación, de manera que los niveles estacionarios de acetilación de las histonas depende del balance de las actividades HAT y HDAC. Al igual que para las HATs, también se ha descrito que las HDACs pueden desacetilar proteínas no histona como p53, E2F, $\alpha$-tubulina y MyoD [209,521]. La clasificación de los diferentes 
tipos de HDACs es confusa, pero generalmente se aceptan cuatro clases de HDACs: las tipo Rpd3p o clase I; las tipo Hda1p o clase II; las tipo Sir2p dependientes de $\mathrm{NAD}^{+}$y las tipo HD2 [292]. Rpd3p y Hda1p son desacetilasas de acción global que afectan a muchos promotores y regulan así la transcripción de muchos genes [386]. Las proteínas Hos1p y Hos2p (HDA one similar) de levaduras pertenecen a la subfamilia de enzimas tipo Rpd3p. Hos3p es una HDAC que a pesar de haberse identificado en diferentes organismos, no encaja exactamente ni en el grupo de HDACs tipo Rpd3p ni en el tipo Hda1p. Por este motivo unas veces se ha clasificado como una HDAC de clase II y otras se ha situado entre las dos clases, aunque en realidad no parece correlacionar bien con ninguna de las dos categorías. Característico de estas enzimas a la resistencia a inhibidores como la Tricostatina A (TSA), que afectan a HDACs de clase I y II [55].

Las histona desacetilasas participan en la regulación de la transcripción génica, pero también están implicadas en la regulación de otros procesos como el ciclo de división y la proliferación celular y el desarrollo de cáncer, lo que ha dado lugar a una búsqueda y caracterización exhaustiva de inhibidores de HDACs [414]. Al igual que otros complejos implicados en la regulación de muchos procesos celulares vitales, las actividades HDACs se encuentran finamente reguladas por múltiples mecanismos. La actividad desacetilasa de las proteínas HDAC depende, entre otras cosas, de interacciones proteína-proteína específicas, de manera que las subunidades catalíticas requieren otras subunidades o cofactores para ser activas (la mayoría de las enzimas recombinantes son inactivas). Además las HDACs son reguladas por modificaciones postraduccionales, a nivel de expresión y por disponibilidad de cofactores. Por último cabe resaltar la importancia de la regulación a nivel de localización subcelular.

En relación con la desacetilación específica, se ha descrito que el complejo HDA1 de S. cerevisiae es dirigido a través del represor general ScTup1p al promotor del gen ScENA1, gen que codifica una ATPasa de membrana involucrada en el transporte de sodio desde el citoplasma al exterior celular. In vitro, se ha demostrado que ScTup1p interacciona con ScHda1p y ScHda3p pero no con ScHda2p. El anclaje de ScTup1p a secuencias específicas del promotor de ScENA1 (URS, upstream repressor sequences) es llevada a cabo a través de las proteínas de unión a DNA ScSko1p y ScMig1p [515]. La actividad de ScHda1p en el promotor de ScENA1 causa la desacetilación local de la cromatina en una región 
que incluye los dos nucleosomas situados aguas abajo del sitio de unión del complejo [515]. Otro de los genes cuya expresión se encuentra alterada en el mutante nulo Schda1 es ScFLO11, gen que codifica una proteína de la superficie celular relacionada con adhesividad y formación de pseudohifas. La represión de este gen depende de su propio promotor y de la posición que ocupa en el genoma, dado que su inserción en otra región resulta en la pérdida de la regulación. ScHda1p podría relacionarse con la regulación a nivel del promotor de este gen, teniendo en cuenta que ScTup1p es dirigida al promotor de ScFLO11 por medio de la proteína de unión a DNA ScSfl1p y se ha demostrado que ScTup1p puede interaccionar físicamente con ScHda1p. Basándose en estas observaciones se ha propuesto que ScSfl1p podría dirigir a ScTup1p junto a ScHda1p al promotor de ScFLO11 [173]. Además se ha sugerido que ScTup1p podría dirigir a ScHda1p a promotores de otros genes necesarios para los procesos de conjugación, utilización de glucosa y oxígeno, respuesta a estrés osmótico y a daños en el DNA [524]. En S. cerevisiae se ha estimado que aproximadamente el 30\% de los genes reprimidos por ScTup1p son reprimidos parcialmente por ScHda1p [165]. Como se ha indicado anteriormente, ScTup1p puede llevar a cabo su papel como represor por otros mecanismos diferentes a la interacción con histona desacetilasas, como la interacción directa con la maquinaria de transcripción o la modificación de la posición de los nucleosomas. Estos mecanismos parecen solaparse en algunos genes, dado que se ha demostrado que al combinarse las mutaciones en genes que intervienen en cada uno de los tres procesos se detecta desrepresión de genes que no se encontraban en los mutantes sencillos y que si se encuentran desreprimidos en ScTup1p [165,515,538].

\section{Desacetilación global por ScHda1p.}

Al igual que en el caso de otras HDACs, se ha demostrado que ScHda1p participa en la desacetilación de regiones amplias de la cromatina y en la desacetilación inespecífica a lo largo de todo el genoma [252].

La utilización de microarrays de acetilación ha permitido definir dominios subteloméricos del genoma de $S$. cerevisiae que se ven afectados por la actividad de ScHda1p y que se han denominado regiones HAST (Hda1p-affected subtelomeric domains) [386]. Estos dominios son regiones continuas de cromatina (entre 4 y $34 \mathrm{~kb}$ ) que se encuentran cerca de los extremos de la mayoría de los 
cromosomas, adyacentes a la heterocromatina telomérica [252].

Las regiones subteloméricas afectadas por ScHda1p contienen una elevada proporción de genes relacionados con el metabolismo de carbohidratos, especialmente genes que intervienen en gluconeogénesis y crecimiento en condiciones adversas como escasez de nutrientes, choque osmótico o baja concentración de oxígeno en el medio. Dichos grupos de genes se encuentran habitualmente reprimidos en medios ricos por mecanismos que involucran a ScTup1p y su expresión se libera en condiciones de estrés nutricional $[118,252,386,524,525]$.

Dentro de las regiones HAST se encuentra el gen ScFLO11, que como indicábamos anteriormente, es un gen cuya represión requiere ScHda1p. La relación entre ScHda1p y la expresión de ScFLO11, además de explicarse a través de interacciones con ScTup1p y con el promotor del gen, podría explicarse a través de la acción de ScHda1p sobre todo el dominio HAST. Esta observación está de acuerdo con el hecho de que el silenciamiento de ScFLO11, además de ser dependiente del promotor es dependiente de su localización en el genoma [173].

Se ha observado así mismo que la deleción de ScHDA1 causa un incremento de acetilación en una región de $4.25 \mathrm{~kb}$ que incluye el gen ScPHO5, a cuyo promotor ScHda1p no esta específicamente dirigida. En ausencia de la histona desacetilasa ScHda1p, el gen ScPHO5, una vez que desaparecen las condiciones que inducen su expresión, muestra una cinética de reversión al estado de represión mas lenta que la cepa silvestre, por lo que se ha propuesto que una de las posibles funciones de la desacetilación global por ScHda1p es permitir una rápida conversión al estado de represión [489].

Por último, ScHda1p interviene en la desacetilación de histonas a lo largo de todo el genoma, acción global que se ha sugerido podría ser importante para mantener los niveles de expresión basal [489,524]. Se ha observado que ScHda1p se une a regiones codificantes que están preferentemente hipometiladas en el residuo lys 4 de la histona $\mathrm{H} 3$ y se ha demostrado que existe correlación entre la metilación de estos genes y la actividad transcripcional, por lo que se ha propuesto que la metilación en estas regiones podría favorecer la transcripción al impedir la unión de los complejos de desacetilación [31]. Por otro lado, se ha observado en diferentes especies que la desacetilación del aminoácido lys 9 de la histona H3, 
llevada a cabo por la proteína homóloga a ScHda1p, estimula la metilación de DNA, favoreciendo la formación de la heterocromatina, donde la transcripción se mantiene silenciada [341,524].

Dado que in vitro ScHda1p no tiene actividad histona desacetilasa por si misma, es probable que también ScHda2p y ScHda3p intervengan en los procesos de desacetilación global.

\section{Poliaminas}

Las poliaminas putrescina, espermidina y espermina son compuestos nitrogenados alifáticos que actualmente se consideran como reguladores del crecimiento y desarrollo tanto en plantas, animales y microorganismos por su efecto demostrado sobre el crecimiento, la división y la diferenciación celular a bajas concentraciones. Por su carácter policatiónico pueden unirse a moléculas cargadas negativamente tales como ácidos nucleicos, proteínas o fosfolípidos, alterando la expresión génica y la actividad de ciertos enzimas, así como variando la fluidez y la permeabilidad de las membranas biológicas. En algún caso, las poliaminas actúan como reserva de nitrógeno, constituyendo la única fuente del mismo.

La participación fisiológica de las poliaminas ha sido conocida mediante la realización de estudios en células incapaces de biosintetizar poliaminas, empleando inhibidores de la síntesis de estos compuestos y usando animales de laboratorio, como ratones transgénicos.[491]. Con estas investigaciones se ha comprobado que las poliaminas son compuestos indispensables para el crecimiento y el buen funcionamiento celular. Las propiedades de las poliaminas se deben principalmente a que cuando se encuentran en $\mathrm{pH}$ fisiológico los grupos amino están cargados positivamente, lo que les permite interactuar con grupos aniónicos de las proteínas, de los ácidos nucleicos y de los fosfolípidos, para proporcionarles estabilidad en su estructura [66]. La ornitina descarboxilasa (ODC), es una enzima presente en todos los organismos, participa en procesos de transición dimórfica, de diferenciación celular y crecimiento celular [170,221]. El análisis de su secuencia de aminoácidos muestra zonas altamente conservadas en organismos eucarióticos incluyendo a los hongos, es la enzima clave del metabolismo de las poliaminas y está altamente regulada transcripcional, traduccional y postraduccionalmente [468]. El inhibidor 1,4-diamino-2-butanona (DAB) es ampliamente utilizado para abatir la actividad de la ODC inhibiendo la 
transición dimórfica levadura-hifa. Estos datos han sido reportados para Candida albicans, Mucor rouxii, Yarrowia lipolytica, Paracoiccidiodes brasiliensis y Ustilago maydis [170,310,402]. La delecion del gen que codifica para la ornitina descarboxilasa $O D C 1$ en $C$. albicans habia sido previamente realizado en nuestros laboratorio [188]. En el nuestro trabajo se demostro que en ausencia de Odc1p en C. albicans se puede modular la transición levadura-hifa modulando nivel de poliaminas bajo $0.01 \mathrm{mM}$ o alto $10 \mathrm{mM}$ [188]. 


\section{Objetivos}

La plasticidad morfológica de C. albicans constituye un importante factor de virulencia de este patógeno fúngico. La transición levadura-hifa es además un proceso de especial interés para constituir un modelo de diferenciación celular. Las diferentes rutas de señalización que regulan la formación de hifas en $C$. albicans han sido ampliamente estudiadas. Sin embargo, no se ha determinado aún como se producen los cambios en la expresión génica en el contexto de la cromatina durante la transición dimórfica, así como tampoco se ha podido establecer claramente la unión entre las rutas de transducción de señales y los procesos de elongación celular. Considerando que los procesos de acetilación y desacetilación de histonas constituyen uno de los mecanismos de regulación de la expresión génica en organismos eucariotas, nos planteamos los siguientes objetivos:

1. Analizar el perfil de transcripción de la cepa CAI4 durante la transición levadurahifa.

2. Determinar bajo diferentes condiciones de inducción si la histona acetiltransferasa Gcn5p y la histona desacetilasa I Hda1p de C. albicans, son necesarias para el desarrollo de procesos morfogenéticos tales como son la formación de hifas, pseudohifas y clamidosporas.

3. Analizar el papel de Gcn5p y Hda1p el doble mutante en la regulación de la expresión de genes que codifican factores de transcripción claves en el proceso de transición levadura-hifa.

4. Analizar el perfil de transcripción del mutante odc1/odc1 durante el crecimiento de forma de levadura y durante la transición levadura-hifa a bajas $(0.01 \mathrm{mM})$ y altas $(10 \mathrm{mM})$ concentraciones de putrescina.

5. Determinar bajo diferentes condiciones de inducción si las histonas acetiltransferasas de $C$. albicans, CaGcn5p y CaHat1p, son necesarias para el desarrollo de procesos morfogenéticos, como son la formación de hifas, pseudohifas y clamidosporas.

6. Analizar el perfil transcripcional del mutante doble gcn5, hat1 durante el crecimiento de forma de levadura. 


\section{Materiales y Métodos}

\section{Medios de cultivo y condiciones de crecimiento}

\section{Medio de cultivo para E. coli}

Las bacterias se crecieron en medio Luria-Bertani o LB $1 \%(p / v)$ bactotriptona, 0.5 $\%(p / v)$ extracto de levadura, $1 \%(p / v) \mathrm{NaCl}$.

\section{Medios de cultivo para C. albicans}

Los medios de cultivo empleados para el crecimiento de levaduras fueron: medio rico o YEPD 1\% (p/v) extracto de levadura, 2\% (p/v) bactopeptona, 1\% (p/v) glucosa]; medio mínimo sintético o YNB $0.67 \%$ ( $p / v)$ Yeast Nitrogen Base sin aminoácidos, $1 \%(\mathrm{p} / \mathrm{v})$ glucosa. Los medios de cultivo sólidos se prepararon de la misma manera, pero añadiendo agar al $2 \%(p / v)$. Los cultivos en medio líquido de C. albicans se realizaron en matraces Erlenmeyer. El volumen del medio fue siempre inferior a un tercio del volumen del matraz. Las incubaciones se llevaron a cabo en agitadores orbitales a $200 \mathrm{rpm}$ y a $28^{\circ} \mathrm{C}$. El crecimiento se determinó midiendo la absorbancia en un espectrofotómetro a una longitud de onda de $600_{\mathrm{nm}}$, o mediante el recuento de células en una cámara Thoma. De forma rutinaria, para los inóculos se estimó la cantidad de células considerando la correspondencia entre un valor de D.O. de 0.4 con una concentración de $1 \times 10^{7}$ células $/ \mathrm{ml}$.

En todos los casos las células en los medios sólidos utilizados para la inducción de crecimiento filamentoso se sembraron a razón de 50 ufc por placa y se incubaron a $37^{\circ} \mathrm{C}$, excepto en el caso del medio embedded en el que la temperatura de incubación fue de $28^{\circ} \mathrm{C}$.

El medio empleado para la inducción de clamidosporas fue cornmeal agar al 1.7\% (Difco). Después de disolver y autoclavar el medio se añadió Tween 80 (Sigma) hasta una concentración final del $0.33 \%$. Las células de las diferentes cepas de $C$. albicans se sembraron en estrías, sobre ellas se dispusieron cubreobjetos con el fin de crear condiciones de crecimiento microaerófilas. Las placas se incubaron en oscuridad a $25^{\circ} \mathrm{C}$, durante 14 días. Se tomaron muestras de cada una de ellas a los 7 y 14 días de crecimiento. Para mejorar la visualización de las clamidosporas al microscopio óptico, las muestras se sometieron a una tinción con lactofenol-azul algodón. Las cepas obtenidas y empleadas en el presente trabajo se conservaron mediante resiembras periódicas en placas de medio sólido. Las placas inoculadas se incubaron a $28^{\circ} \mathrm{C}$ durante 48 horas en el caso de $\mathrm{C}$. albicans y a $37^{\circ} \mathrm{C}$ durante 
12 horas en el caso de E. coli. Posteriormente se conservaron a $4^{\circ} \mathrm{C}$. Con la finalidad de mantener las células durante largos periodos de tiempo, los microorganismos se almacenaron en viales con glicerol al $30 \%$ en ultracongeladores a $-80^{\circ} \mathrm{C}$. En el presente trabajo se llevó a cabo la transformación de $C$. albicans mediante la adición de DNA lineal o DNA plasmídico a protoplastos de esta levadura, que se obtuvieron de forma previa a cada transformación. Dado que en todos nuestros experimentos de transformación se utilizó el gen CaURA3 como marcador de selección, las cepas a transformar fueron siempre Ura (CAl4 y derivadas).

\section{Detección de secuencias de DNA específicas (Southern-blot)}

Una vez digerido el DNA genómico de C. albicans $(15-20 \mu \mathrm{g})$ con las enzimas de restricción adecuadas, los fragmentos de DNA se separaron, junto a un marcador de peso molecular, mediante electroforesis en geles de agarosa al 1\%. El DNA separado se visualizó por la incorporación en el gel de bromuro de etidio y se fotografió junto a una regla con el fin de tener una referencia que permitió posteriormente determinar el tamaño de las bandas hibridadas. La hibridación se realizó en una solución de igual composición a la de prehibridación y a la que se añadió la sonda radiactiva previamente desnaturalizada. La membrana se dejó hibridando durante aproximadamente 16 horas a $65^{\circ} \mathrm{C}$. A continuación se realizaron varios lavados de tiempo variable con $100 \mathrm{ml}$ de las soluciones de los lavados. Los filtros se analizaron con el sistema de detección de radiación, captura y análisis de imágenes BAS-1500 Storage Phosphor Imaging System (Fujifilm). Para ello cada membrana se expuso en oscuridad a una pantalla del sistema (cubierta por una capa de fósforo foto-estimulable), durante un período de tiempo que osciló entre 1 y 12 horas. Posteriormente la superficie de la pantalla fue escaneada y se obtuvo una imagen que reveló la impresión causada por la radiación, permitiendo estimar el tamaño de los fragmentos de DNA que habían hibridado específicamente con la sonda.

\section{Análisis de la sensibilidad a compuestos relacionados con estructura de la pared celular}

A partir de células cultivadas hasta fase exponencial, se prepararon suspensiones a una D.O. $600 \mathrm{~nm}$ de 0.4 y a partir de ellas se realizaron tres diluciones seriadas de 
$1 / 10,1 / 100$ y $1 / 1000$. Se tomaron $5 \mu l$ de cada una de las diluciones y se sembraron en placas de medio mínimo conteniendo diferentes concentraciones de Ios compuestos a analizar: Higromicina; Itraconazol, Flocunazol, $\mathrm{NaCl}$, Calcofluor white, Cafeína y SDS. Las diferencias en el crecimiento de las distintas cepas se valoraron después de 72 horas de incubación a $28^{\circ} \mathrm{C}$. El tiempo de incubación se prolongó en determinados casos con el fin de apreciar mejor la variación en el crecimiento.

\section{Ensayo de afinidad a Alcian blue}

El Alcian blue es un colorante catiónico no fluorescente que se une específicamente a las cadenas de fosfomanosas presentes en la superficie celular de las levaduras $[20,189]$. Este ensayo permite detectar cambios en la síntesis y secreción de las manoproteínas que forman parte de la pared celular. Las células recogidas en fase exponencial se lavaron 2 veces con $\mathrm{HCl} 0.02 \mathrm{~N}$ y a continuación se prepararon 3 diluciones de $1 \mathrm{ml}$ a una D.O. de 2.5 de cada una de las cepas. Las muestras se centrifugaron resuspendiendo las células en $1.5 \mathrm{ml}$ de una solución de Alcian blue $0.005 \%$. Tras 10 minutos de incubación a temperatura ambiente, las muestras se centrifugaron 3 minutos a la máxima velocidad y seguidamente se midió la absorbancia a $600_{\mathrm{nm}}$ de los sobrenadantes. La concentración de Alcian blue de cada sobrenadante se determinó por comparación con una recta patrón que permitió relacionar la concentración de colorante con la absorbancia y cuyo coeficiente de correlación fue 1.00. A partir de esta concentración se estimó el porcentaje fijado por cada cepa considerando como $100 \%$ la concentración de Alcian blue del sobrenadante de una muestra tratada de la misma forma pero en la que no se incluyeron células.

\section{Ensayo de virulencia}

Se prepararon cultivos de cada una de las cepas de C. albicans a ensayar en 100 $\mathrm{ml}$ de YEPD a una D.O. inicial de 0.03. Se incubaron durante aproximadamente 12 horas a $28^{\circ} \mathrm{C}$ con el fin de obtener células en forma de levadura y en fase exponencial de crecimiento. Las células se recogieron, se lavaron dos veces con PBS $1 \times$ y se resuspendieron a una concentración de $5 \times 10^{8}$ células $/ \mathrm{ml}$. La concentración celular de la suspensión final se verificó en una cámara Thoma y por absorbancia a $600_{\mathrm{nm}}$. A partir de dicha suspensión se inocularon por vía 
intraperitoneal 10 ratones CD1 (machos de aproximadamente $20 \mathrm{~g}$ de peso) con $5 \times 10^{8}$ células de $C$. albicans cada uno. A partir del día de infección se realizó un seguimiento diario de la supervivencia de los ratones infectados que se prolongó hasta el día 30.

\section{Comparación de perfiles de transcripción mediante microarrays de cDNA.} Aislamiento de RNA total

Las células se recogieron por centrifugación a 3000 rpm durante 5 minutos a temperatura ambiente, los sobrenadantes se eliminaron mientras que el precipitado de células se resuspendió en el volumen residual de medio que quedó en el tubo de centrifugación. Seguidamente las células se congelaron dejando caer gotas de aproximadamente $20 \mu$ en eppendorfs con nitrógeno líquido. Los tubos se mantuvieron en un recipiente con nitrógeno líquido para evitar la descongelación de las muestras, o bien se guardaron a $-80^{\circ} \mathrm{C}$ hasta su utilización. La lisis celular se llevó a cabo de forma mecánica dentro de una cámara de teflón en la que se introdujo, junto a la muestra, una esfera de tungsteno $(7 \mathrm{~mm}$ de diámetro) que se sometió a agitación en un Micro-Dismembrator (Braun, Melsungen).

\section{Marcado, síntesis de cDNA e hibridación}

Para el marcado con Cianina 3-dCTP o Cianina 5-dCTP (fluorocromos que absorben y emiten fluorescencia a diferentes longitudes de onda) se siguió el método de marcaje directo según el protocolo proporcionado por la casa comercial Eurogentec y en el que se introdujeron pequeñas modificaciones. De forma general puede decirse que se trata de una reacción de retrotranscripción en la cual se incorporan los nucleótidos marcados durante la síntesis de las moléculas de cDNA. En cada reacción se utilizaron $15 \mu \mathrm{g}$ de RNA total a los que se añadió $1 \mu \mathrm{l}$ del inhibidor de ribonucleasas RNAsin (Promega) y agua destilada tratada con DEPC hasta completar un volumen de $21 \mu \mathrm{l}$. Las soluciones de RNA se dejaron en un baño a $42^{\circ} \mathrm{C}$ mientras se mezclaron el resto de componentes de la reacción $5 \mathrm{x}$ First-strand buffer (Invitrogen) (8 $\mu \mathrm{l})$, C. albicans specific primer mix (Eurogentec) (0.1 pmoles/ $\mu \mathrm{l})(1 \mu \mathrm{l})$, Oligo dT (12-18) (Sigma) $(0.5 \mu \mathrm{g} / \mu \mathrm{l})(1 \mu \mathrm{l})$, Mezcla de dNTPs excepto dCTP (Sigma) (10 mM) (3 $\mu \mathrm{l})$, dCTP (Sigma) (1 mM) (1 $\mu \mathrm{l})$, Cianina 3dCTP o Cianina 5-dCTP (1 mM).(CyDye TM Fluorescent nucleotides Cy3 o Cy5, 
Amersham) $(1.5 \mu \mathrm{l})$, DTT $(0.1 \mathrm{M})$ (Invitrogen) $(4 \mu \mathrm{l})$. La mezcla se realizó en eppendorfs cubiertos con papel de aluminio para evitar la exposición a la luz de los fluorocromos. La solución de RNA se añadió a la mezcla de reactivos y seguidamente se incubó 5 minutos a $65^{\circ} \mathrm{C}$ y 5 minutos a $42^{\circ} \mathrm{C}$. Se añadió $1 \mu \mathrm{l}$ de transcriptasa reversa (Invitrogen) y se incubó 3 horas a $42^{\circ} \mathrm{C}$, añadiendo $1 \mu \mathrm{l}$ adicional de enzima transcurrido la mitad del tiempo de incubación. La reacción se detuvo añadiendo $5 \mu \mathrm{l}$ de EDTA pH 8.0 y $2 \mu \mathrm{l}$ de $\mathrm{NaOH} 10 \mathrm{~N}$ e incubando la muestra 20 minutos a $65^{\circ} \mathrm{C}$. A continuación, el pH se bajó con la adición de $4 \mu \mathrm{l}$ de una solución de ácido acético $5 \mathrm{M}$. La purificación de las moléculas de cDNA sintetizadas se llevó a cabo utilizando el kit de purificación de productos de PCR QIA-kit PCR purification kit (Quiagen) siguiendo el protocolo descrito por la casa comercial. Después de dos pasos de elución con $50 \mu \mathrm{l}$ de agua destilada cada uno, se cuantificó el cDNA sintetizado, la cantidad de fluorocromo incorporado y la frecuencia de incorporación del mismo según las fórmulas que se indican a continuación: Dada la baja concentración de cDNA que se obtiene, la absorbancia de la muestras se midió directamente, sin diluciones, utilizando ultramicrocubetas (Hellma). Considerando que Eurogentec advierte que no deben usarse muestras con una f.o.i $\leq 10$ y que los valores óptimos se encuentran entre 20 y 50 , en nuestros experimentos decidimos usar solo aquellas muestras con una f.o.i. $\geq 16$, con el fin de asegurar buenos resultados. El volumen de muestra correspondiente a 65 pmoles de fluorocromo se concentró utilizando filtros microcon-30 (Amicon) hasta conseguir un volumen final de 5 a $10 \mu \mathrm{l}$. Para cada hibridación se mezclaron las muestras de cDNA procedentes de cada una de las dos cepas a comparar (marcada una con Cy3 y otra con Cy5) y $5 \mu$ de DNA de esperma de salmón (Sigma-Aldrich) previamente desnaturalizado por calor. El cDNA se desnaturalizó manteniendo la muestra 2 minutos a $95^{\circ} \mathrm{C}$ y enfriándola inmediatamente en hielo, seguidamente se añadió tampón de hibridación (Dig Easy Hybridization buffer, Roche) hasta un volumen final de $60 \mu \mathrm{l}$. La mezcla se depositó entonces sobre un cubreobjetos de plástico $(60 \times 24 \mathrm{~mm}$, Sigma-Aldrich), el microarray se puso en contacto con la muestra por la cara que contenía el DNA y una vez que la muestra se extendió por aproximadamente la mitad del cubreobjetos se dio la vuelta al microarray y se colocó en una cámara de hibridación (Corning), que se sumergió en un baño a $42^{\circ} \mathrm{C}$. Después de 12 horas de hibridación, se retiró el cubreobjetos introduciendo el microarray en una cubeta con la primera solución de lavado (SDS 
1\%/SSC 2x), se transfirió a un tubo falcon con la misma solución donde se mantuvo 5 minutos a temperatura ambiente con agitación ocasional y protegido de la luz. Para eliminar los restos de SDS, el microarray se pasó sucesivamente por dos tubos falcon con SSC 2x, en el último de ellos se mantuvo 5 minutos con agitación ocasional. Por último el microarray se secó mediante centrifugación a 2000 rpm durante 5 minutos a temperatura ambiente. Los microarrays de $C$. albicans utilizados en el presente trabajo fueron sintetizados por la empresa Eurogentec en colaboración con el consorcio europeo Galar Fungail Group (www.pasteur.fr/recherche/unites/Galar Fungail), al cual pertenece nuestro grupo de investigación. El contenido y la forma de fabricación se describen a continuación y pueden encontrarse en la página www.eurogentec.com. La información sobre las secuencias codificantes se obtuvo de la base de datos CandidaDB (www.pasteur.fr/recherche/unites/GalarFungail/CandidaDB) desarrollada por el consorcio Galar Fungail Group partiendo de la secuencia completa del genoma de la cepa SC5314 de C. albicans publicada por el Stanford Genome Technology Center [96]. A partir de dichas secuencias se diseñaron los oligonucleótidos que permitieron amplificar una región específica de cada ORF, generalmente correspondiente a la región $3^{\prime}$ de la ORF del gen. Se ha llevado a cabo la comparación de los perfiles de transcripción de dos mutantes de $C$. albicans con el de la cepa parental, CAI4, en cuatro condiciones de crecimiento: fase exponencial como levaduras, y a tres tiempos (15, 60 y 180 minutos) de la inducción de la formación de hifas, en medio Lee a $37^{\circ} \mathrm{C}$. Para cada comparación mutante vs. CAl4 se han realizado cuatro hibridaciones correspondientes a dos replicados biológicos, en los que el RNA de la cepa mutada procede de cultivos independientes, y dos hibridaciones para cada uno de ellos en los que el cDNA se marcó de forma inversa (dye swap). El cDNA de la cepa parental, CAI4, se sintetizó en cada condición a partir de una mezcla de RNA obtenido de diferentes cultivos de esta cepa provenientes de tres colonias. Considerando que para cada comparación se hibridaron 4 microarrays y que cada gen se encuentra depositado por duplicado en cada microarray, para la mayoría de los genes se analizaron 8 datos para cada condición. 


\section{Resultados}

\section{Respuesta de la cepa CAI4 de Candida albicans a la temperatura}

Uno de los parámetros involucrados en transición levadura-hifa es la temperatura y su cambio esta implicado a patogénesis.

El análisis transcripcional de $C$. albicans en respuesta a la transición levadura-hifa después de 15,60 y 180 min de exposición a medio Lee a $37^{\circ} \mathrm{C}$ fue llevada a cabo para identificar los genes expresados diferencialmente en la cepa CAl4. Por el método de microarrays de cDNA, se encontraron 341, 405 y 251 genes expresados diferencialmente en cada uno de los tiempos analizados. El nombre y la función de cada uno de ellos se han incluido en las Tablas 2.1-2 del material suplementario. En conjunto se detectó que la expresión de 783 genes (403 sobrey 380 sub-expresados) es afectada por cambio de la temperatura en al menos uno de los tres tiempos de inducción analizados. La comparación de los genes expresados diferencialmente en cada uno de los tiempos mediante diagramas de Venn señala que los resultados de expresión diferencial varían a lo largo de la inducción (Figura 2.3). Como se ha señalado en le Figura 2.2, se ha detectado claramente la expresion de un número significativo de genes después de 60 minutos, lo que sugiere que la mayoría de las señales y los genes reguladores que participan en la morfogenesis realiza su función rápidamente. Se observó una buena correlación estadística en nuestros resultados 43 genes son comunes a los tres tiempos ensayados (24 sobre- y 19 sub-expresados), 68 genes en los 15 y 60 min (31 sobre- y 37 sub-expresados) y 52 genes en 60 y 180 min. (40 sobre- y 12 sub-expresados). 222 genes (75 sobre- y 147 sub-expresados), 240 genes (155 sobre- y 85 sub-expresados), y 148 genes (74 sobre- y 74 sub-expresados) solo varían en un tiempo de inducción (15, 60 y 180 min. respectivamente). Sin embargo, únicamente 8 genes eran comunes entre los 15 y 180 min lo que muestra la coherencia de nuestro experimento. Los 43 genes comunes a los tres tiempos se describen en la Tabla 2.1. entre ellos se ha detectado la expresión diferencial de varios genes relacionados con pared celular, como HWP1 [443]; ECE1 [32]; PHR1 [131], RBT1 [434], YWP1[159] y PIR1 [309]. Todos estos genes se han descrito anteriormente como sobre y sub-expresados en niveles similares [226,342] y están reguladas por Efg1p y Tup1p [108,226,434]. En el resto de las categorías funcionales las más representativas son transporte con 9 genes, incluido dos de transporte de oligopéptidos, oxidasa/peroxidasa (4 genes), glicólisis 
(3 genes), y dehidrogenases (3 genes). En general, nuestros resultados coinciden con lo descrito anteriormente por varios autores[226,334,342,427], con una sola excepción; no hemos podido detectar la inducción de los genes de la familia de SAP. Esto se debe probablemente a las condiciones que hemos utilizado, mientras que otros autores han utilizado diferentes medios inductores: medio YPD y Lee con suero al $4 \%$. Nuestro resultado está respaldado por el hecho de que el único factor de transcripción que estaba regulado en los tres tiempos es STP3 [311]. Para analizar el resto de los genes expresados diferencialmente durante la transición dimórfica se agruparon por la similitud de sus patrones de expresión diferencial a lo largo del tiempo, mediante un análisis tipo Cluster en el programa GeneSpring 5.0. De esta forma se analizaron por separado aquellos grupos representativos cuya expresión cambiaba al menos en un tiempo de incubación. Se realizaron diferentes distribuciones en un número variable de grupos, y se observó que se podian: pared celular (famila de ALS, glucanasas, glucosidasas y mannosiltransferasas, GPI-anchored proteína), genes implicados en procesamiento de RNA y biogénesis de ribosomas, metabolismo de carbohidratos, transportadores y transportadores de oligopeptidos, resistecia a drogas, oxidasas/peroxidasas, hidrolasas/deshidrogenasas y biosíntesis de tiaminas.

En la Figura 2.4A se ha reflejado la alteración de la expresión de varios genes que codifican proteínas estructurales asociadas de forma preferente a la pared de las hifas como: ALS1, ALS2, ALS3, ALS5, ALS9, PHR1, HWP1, ECE1, $[32,108,226,279,342,370,443]$. En relación con la Figura $2.4 B$ se detectó la sobreexpresión de varios genes implicados en procesamiento de RNA y biogénesis de ribosomas, en la Figura 2.5 se encuentran subexpresados 35 genes relacionados con la ruta de glicolisis. Se ha descrito que la expresión de genes implicados en glicólisis se reduce de forma drástica en el inicio de la transición dimórfica [460] lo que confirma nuestro resultados. En el capitulo II, se ha explicado con más detalles los resultados de este experimento.

\section{Deleción del gen GCN5 en el mutante HDA1 de la cepa de NDH4}

Nuestro grupo de trabajo, está llevando a cabo el análisis sistemático de la implicación de diferentes histonas desacetilasa e histona acetiltransferasas en la regulación de la transición levadura-hifa y de otros procesos morfogenéticos. En la primera parte de está memoria se han expuesto los efectos en morfogénesis de la 
ausencia de una de las probables histonas desacetilasas y de una histona acetiltransferasas de C. albicans, Gcn5p y CaHda1p. La interrupción de los dos alelos del gen GCN5 en la cepa NDH4 (con el gen HDA1 delecionado) se llevó a cabo siguiendo la estrategia del "Ura-blaster" de forma similar a la descrita en nuestro laboratorio. En la Figura 3.1 se muestra la comprobación del genotipo de las cepas generadas mediante experimentos tipo Southern. EI DNA genómico fue digerido con Munl y como sonda se utilizó la secuencia de 465 pb correspondiente a la región 3' no codificante del gen GCN5. Tal y como puede apreciarse en la Figura 3.1, las cepas CAI4, NDH4 y heterocigotas para la deleción del gen presentan la banda de $0.987 \mathrm{~kb}$ correspondiente al alelo silvestre de GCN5. Esta banda no aparece en las cepas homocigotas para la deleción de GCN5 (SGM4-3 y SGM4-11) y en su lugar se detectan otras bandas cuyo tamaño se ajusta a la secuencia del cassette de deleción y de las secuencias flanqueantes.

\section{Efecto de la doble deleción gcn5, hda1 en el crecimiento exponencial}

Mediante el seguimiento de la D.O. alcanzada a lo largo del tiempo por cultivos de las diferentes cepas, se detectó un ligero retraso en el crecimiento del doble mutante gcn5, hda1 y del mutante sencillo gcn5 respecto a la cepa parental CAI4. Se observó que en fase exponencial el tiempo de generación de las cepas SGM4 y RDG4 era mayor al de la cepa NDH4 y la cepa parental CAI4. Estimamos que en este medio las cepas SGM4 y RDG4 iniciaban la fase exponencial tras aproximadamente 22 horas de crecimiento. En relación con este fenotipo, en S.cerevisiae se ha descrito que la cepa homocigota para la deleción del gen ScGCN5 [461] muestra también defectos de crecimiento en medio rico. Después de inocular en medio mínimo a $28^{\circ} \mathrm{C}$ se observó que mientras que en los cultivos de la cepa CAI4 y el mutante simple hda1 las células crecían únicamente como levaduras en los cultivos del doble mutante gcn5, hda1 y mutante simple gcn5 del aparecían pseudohifas y agregados celulares.

\section{Efecto de la doble deleción gcn5, hda1 en la filamentación en medios sólidos}

Se ha analizado el efecto de la doble deleción en la capacidad de filamentación en diferentes medios sólidos y se ha observado que al igual que los dos mutantes simples presenta defectos de filamentación en todos los medios de inducción 
ensayados (Figura 3.5).

\section{Efecto de la doble deleción gcn5, hda1 en la transición dimórfica en medio líquido}

Después de inducir la transición dimórfica en medio líquido Lee a $37^{\circ} \mathrm{C}$ se observó que el mutante doble gcn5, hda1, es incapaz de formar hifas, igual el mutante simple gcn5. Ambas cepas forman en lugar de hifas, pseudohifas y agregados celulares aunque el grado de agregación parece menor en el doble mutante que el mutante simple gcn5. El doble mutante se comporta así, de diferente forma que el mutante simple hda1, ya que en medios líquidos si es capaz de formar hifas. En la inducción en medio Lee a $37^{\circ} \mathrm{C}$ con suero observamos el mismo efecto.

\section{Efecto de las deleción de los genes GCN5 y HDA1 en la formación de clamidosporas}

A continuación se analizó el efecto de la deleción de los genes GCN5 y HDA1 en la formación de clamidosporas. Las cepas se incubaron en medio corn meal agar bajo condiciones microaerófilas y a temperatura $25^{\circ} \mathrm{C}$, hemos observado que la cepa doble mutante gcn5, hda1 no es capaz de formar clamidosporas al igual que el mutante simple gcn5.

\section{Análisis de la sensibilidad de la doble deleción gcn5, hda1 a compuestos que afectan a la pared celular}

Uno de los ensayos utilizados para determinar la existencia de posibles alteraciones en pared celular, fue el ensayo de "Alcian blue". En la Figura 3.10 se muestran los valores del colorante fijado por la cepa CAI4, NDH4, RDG4 y SGM4 de C. albicans. Si tomamos como $100 \%$ la cantidad de colorante fijado por las células de la cepa silvestre, las células del doble mutante fijan un 185,59\%. El porcentaje de colorante por las cepas NDH4 y RDG4 es de un 120,65\% y 155,94\% respectivamente. Además podemos concluir que, en valor absoluto, el mutante simple hda1 y la cepa CAI4 fijan menos colorante que doble mutante gcn5, hda1 y mutante simple gcn5. Estas diferencias se pueden atribuir a que los mutantes, presentan una mayor cantidad de fosfato en las manoproteínas presentes en la pared celular. En C. albicans, la capacidad de adhesión depende de su pared celular [454]. Estudios recientes han revelado que las manoproteínas una vez 
glicosiladas forman una interacción directa con el hospedador por lo que defectos en el proceso de glicosilación son importantes para la virulencia [470]. Durante el proceso de transición levadura-hifa existe un rápida remodelación y expansión de la pared celular durante la formación de hifas; por esta razón, mutaciones en genes que intervienen en la construcción de la pared celular presentan defectos de filamentación [189,470]. Además los genes específicos de hifas en C. albicans codifican proteínas de secreción de la pared celular. Así que, es de esperar que defectos en cualquiera de las rutas de señalización que regulan el proceso de transición de la levadura-hifa, se vean reflejados en una alteración de algunos componentes de la pared celular. Puesto que las cepas NDH4, RDG4 y SGM4 parecían presentar defectos en la pared celular, decidimos estudiar su sensibilidad a zimoliasa (preparación lítica con actividad de $\beta-1,3$ glucanásica y proteásica, que desorganiza y degrada la pared celular y puede ser utilizada para medir cambios en la composición y ensamblaje de la pared celular [375]. Para el ensayo de sensibilidad a zimoliasa, tomamos como referencia estudios previos realizados en nuestro laboratorio [189], en los que se analizó como afectan distintas concentraciones de zimoliasa a la integridad de células CAl4. El resultado obtenido se muestra en la Figura 3.9. Como puede observarse, no se detectó una diferencia significativamente entre la diferencias de la densidad óptica a largo del tiempo en los mutante simple y doble mutante. Por último, decidimos analizar la sensibilidad a otros agentes que afectan a la pared celular: Cafeína, Calcofluor white, $\mathrm{NaCl}$, SDS y Congo red. Como se puede observar en la Figura 3.8, se encontraron diferencias de sensibilidad a estas concentraciones de Calcofluor white entre el doble mutante y el mutante gcn5 con respecto a la sepa CAI4. El doble mutante gcn5, hda1 y el mutante sencillo gcn5 no crecieron en ninguna de las diluciones en placa con Calcofluor white a concentración de $1 \mathrm{mg} / \mathrm{ml}$. Como se pude observar en la Figura 3.8, el doble mutante gcn5, hda1 y el mutante sencillo gcn5 se presentan más sensibles con respecto a las cepas CAl4 y el mutante sencillo hda1. El doble mutante gcn5, hda1 y el mutante gcn5 no crecieron en placa conteniendo cafeína y SDS a una concentración de $15 \mathrm{mM}$ y 0,05\% respectivamente. En S. cerevisiae la hipersensibilidad a cafeína se ha relacionado con defectos en los mecanismos reguladores de la formación de la pared celular. Todos estos procesos parecen estar alterados en la cepa doble delecionada. También se detectaron ciertas diferencias en la sensibilidad a SDS, un detergente 
que altera la membrana plasmática. La cepa doble delecionada parece ser un grado más sensible que la cepa CAI4 y mutante sencillo hda1. La hipersensibilidad a SDS se ha relacionado con la integridad de la pared celular, necesaria para la protección de la célula. Además pueden producirse diferencias en la sensibilidad a este compuesto debidas a alteraciones en la composición de la membrana plasmática.

\section{Analisis del transcriptoma del mutante gcn5, hda1 durante crecimiento de levadura}

Al objeto de analizar los posibles genes regulados por procesos de acetilacion y de deacetilacion se analizó el transcriptoma del mutante doble gcn5, hda1, utilizando como control la cepa CAI4, durante el crecimiento levadura y durante la transición levadura-hifa. Anteriormente en nuestro laboratorio se han llevado a cabo experimentos en los que se ha analizado el transcriptoma de los mutantes simples gcn5 y hda1 tal y como se ha señalado anteriormente, 4 condiciones examinadas, crecimiento exponencial como levaduras (en YNB a $28^{\circ} \mathrm{C}$ ) y a tres tiempos $(15,60$ y 180 minutos) durante la transición dimórfica en medio líquido Lee a $37^{\circ} \mathrm{C}$. A partir de células recogidas en fase exponencial de crecimiento como levaduras, se detectó que se encontraba alterada la expresión de 180 genes por la doble deleción de GCN5 y HDA1 durante el crecimiento en forma de levadura. El número de genes que presentan expresión diferencial en el mutante doble gcn5, hda1 es menor que el detectado en la cepa gcn5 y mayor que el detectado en la cepa hda1. Lo que está de acuerdo con el hecho de que la cepa SGM4 (gcn5, hda1) muestra alteraciones del fenotipo similares al del mutante gcn5. Se detectaron 3 genes comunes entre los mutantes simples y doble. Entre estos genes, ALS4 y $A L S 2$ codifican proteínas de la pared celular, mientras que otro, BGL22, codifica una posible endo $\beta$ 1,3 glucanasa secretada que podría intervenir en remodelación de la pared celular. Estos resultados sugirieren que en condiciones normales estos genes son controlados por separado por Gcn5p y Hda1p. La alteración de la expresión de estos genes permite realizar algunas hipótesis sobre las causas de las diferencias de afinidad a Alcian blue. Las proteínas codificadas por los genes ALS2 y ALS4 pertenecen a la familia de aglutininas ALS. El mecanismo por el cual estas proteínas promueven la adhesión a células del hospedador no se conoce aún bien, pero se ha propuesto que podría deberse a la exposición hacia el 
exterior de su extremo amino terminal. Este dominio presenta características hidrofóbicas y se encuentra menos glicosilado que la región central [200,202]. De forma general, se ha sugerido que la hidrofobicidad celular viene dada por la exposición de proteínas o regiones proteicas poco glicosiladas y el enmascaramiento de proteínas o regiones hidrofílicas más glicosiladas [183]. La reducción de expresión de $A L S 2$ y ALS4 podría suponer así una mayor exposición de proteínas hidrofílicas más glicosiladas, que explicaría también la mayor capacidad de unión a Alcian blue. Además, 47 genes son comunes entre el mutantes simples gcn5 y doble gcn5, hda1. Sin embargo, un subconjunto de 130 genes que se vieron afectados en el doble mutante no sufrió modificaciones en ninguno de los mutantes simples. Estos resultados indican que en la condición de crecimiento de levadura ambos genes regulan conjuntamente un importante grupo de genes. En la Figura 3.12 se ha señalado el número de genes expresados diferencialmente, con un factor de variación de los mutantes simple y doble vs. CAI4 $\geq 1.5$ en cada condición. El nombre y la descripción de cada uno de los genes en el mutante doble se muestran en los archivos 3.1 y 3.2 del material suplementario. El mutante doble gcn5, hda1 muestra defectos en el velocidad de crecimiento lo cual podría ser explicado por disminución en la expresión de algunos genes de la ruta de glicólisis (Tabla 3.3). En nuestros experimentos, hemos observado disminución de la expresión de EFG1. Se ha reportado subexpression de la mayoría de los genes de la ruta de glicólisis así como en genes relacionados en el metabolismo de azúcares de reserva tales como, tetralosa y glucogeno subexpresados en el mutante efg1 [108]. Estos resultados sugieren que existe un control indirecto de la expresión de los genes de la ruta de glicólisis en el mutante doble. Hemos encontrado 3 genes sobrexpresados como CSP37 [416], PGA13 y PGA56 que codifican para proteínas de pared celular lo que sugiere una remodelación de la pared celular en el mutante doble. Otro grupo de genes subexpresados fue el de genes que codifican para proteínas ribosomales (13 genes). Se ha reportado que la expresión de 63 genes de 117 (53\%) son subexpresados durante el crecimiento en forma de levadura en el mutante simple gcn5, esto sugiere que Gcn5 podría ser un regulador de la expresión de genes que codifican para proteínas ribosomales. Se ha detectado dos genes (CDR1 and CDR4) subexpresados, los cuales pertenecen la superfamilia de transportadores de drogas. Se ha reportado la sobreexpresión de CDR1 en cepas resistentes a 
azoles [522]. La subexpresion de los genes CDR1 y CDR4, podría estar relacionada con la sensibilidad del doble mutante a compuestos como anfotericina e higromicina.

\section{Análisis del transcriptoma del mutante gcn5, hda1 durante la transición levadura-hifa}

Se ha detectado alterada la expresión de 664 genes en el mutante doble gcn5, hda1 al menos en una de las tres condiciones de la transición levadura hifa analizadas. Como puede observarse en la Figura 3.13, la mayor parte de los genes aparecen expresados diferencialmente a los 60 minutos de inducción. Tanto por el número de genes expresados diferencialmente como la comparación de los patrones de expresión, se puede apreciar una marcada diferencia entre los resultados que se han obtenido en los mutantes simples y los tres tiempos del mutante doble. Además durante la inducción, en el mutante doble también se observan diferencias entre los sucesivos tiempos. Estos resultados indican que, estas variaciones pueden deberse a la modulación de la expresión que sufren numerosos genes por ambas proteínas durante la transición dimórfica. 293, 310 y 238 genes se expresaban diferencialmente en cada uno de los tiempos analizados. El nombre y la función de cada uno de ellos se han incluido en las Tablas 3.3 y 3.4 del material suplementario. En conjunto se detectó la expresión diferencial de 664 genes (312 sobre- 352 sub-expresados) en el mutante doble gcn5, hda1 en al menos uno de los tres tiempos de inducción analizados. Se observó una buena correlación estadística en nuestros resultados, 39 genes comunes en los tres tiempos ensayados (20 sobre- y 19 sub-expresados), 35 genes entre los 15 y 60 min (12 sobre- y 23 sub-expresados) y 33 genes entre 60 y $180 \mathrm{~min}$. (17 sobre- y 16 sub-expresados). 188 genes (72 sobre- y 116 sub-expresados), 203 genes (82 sobre- y 121 sub-expresados), y 135 genes (84 sobre- y 51 sub-expresados) sólo varían en un tiempo de inducción (15, 60 y 180 min. respectivamente). Sin embargo, sólo 31 genes eran comunes entre los 15 y 180 min. Los 39 genes comunes a los tres tiempos se describen en la Tabla 3.4. La categoría principal corresponde a proteínas de pared celular e incluye la sobreexpresión de $R D H 3$ [61,104,342], PIR1, una proteína estructural de pared [309], KRE1 [36,46] y CHR11 [6,61], y la subexpresión de genes específicos de la fase hifa, como ECE1, [27,32]; HWP1, [443]; y dos genes que pertenecen a la familia ALS (ALS1 y ALS3). Previamente se ha reportado que estos genes están sobreexpresados 0 subexpresados durante la transición levadura-hifa [39,342] y la cepa CAl4 (Capitulo II). Se observó que la expresión de los genes CHR11, RDH3, RBT5, 
PGA62 aumenta en ambos mutantes simples, mutante doble y durante la transición levadura hifa de la cepa CAI4 mediada por la temperatura. Lo que sugiere que la expresión de estos genes es regulada por la temperatura y no por procesos de acetilación y deacetilación. Además se detectó que la expresión de los genes PHR1 PGA59 y KRE1 aumenta en la cepa CAI4, en el mutante simple gcn5 y en el mutante doble, y disminuye en el mutante hda1 lo que sugiere que es regulada especialmente por el gen HDA1 (Figura 3.14). Otros genes regulados durante los tres tiempos de incubación pertenecen a las siguientes categorías: transportadores (9 genes), factores de transcripción (5 genes), y biosíntesis de vitaminas (2 genes). Como se mencionó anteriormente, el mutante doble no fue capaz de formar hifas bajo condiciones de inducción, por lo que la subexpresión de estos genes es consistente con nuestros resultados. Además el análisis de los microarrays mostró la sobreexpresion de cinco factores de transcripción, BDF1 [28,302], GAL4 [306], NRG1 [330,331], TYE7 [177,231,281,342] y ZCF39 (Tabla 3.4). Hemos encontrado 16 genes (12-sobre y 4-subexpresados) también pertenecientes a esta categoría (factores de transcripción) durante uno o dos tiempos de inducción (Tabla suplementaria 3.3 y 3.4). Siete de estos genes fueron comunes con el mutante simple gcn5 y el resto podrían estar reguladas por las proteínas Gnc5p y Hda1p. Además hemos observado que 12 genes que codifican para glucanasas, glucosidasas y manosiltransferasas estan regulados a lo largo de la inducción (Tablas suplementaría 3.1 y 3.2 y Figura 3.14). Cinco de estos genes están sobreexpresados (CA5339, MNN4, MNT2, BGL2 y GSL22), y siete subexpresados (MNT1, GSC1, GLC3, SUN41, MNT3, PMT2 y PMT4). El cambio en la expresión de estos genes podría estar relacionado con cambios en la composición de la pared celular. Adicionalmente se encontró que algunos de estos genes son comunes entre los mutantes simple y doble. En el capitulo III, se ha explicado con más detalles algunos grupos de genes cuya expresión ha sido modificada por la deleción de GCN5 y HDA1.

\section{Analizar del transcriptoma del mutante odc1/odc1 durante crecimiento de levadura}

En el capítulo IV nos hemos concentrado en el estudio de los patrones de expresión diferencial en la cepa de C. albicans (odc1/odc1) (agotadas en su reserva interna de poliaminas) suplementando con dos concentraciones de putrescina, concretamente $0.01 \mathrm{mM}$ vs $10 \mathrm{mM}$ durante el crecimiento de levadura y la transición levadura-hifa, en medio Lee a $28^{\circ} \mathrm{C}$ y $37^{\circ} \mathrm{C}$, respectivamente.

Por el método de microarrays de cDNA, se encontraron 137 genes expresados 
diferencialmente en crecimiento de levadura. El nombre y la función de cada uno de ellos se han incluido en la Tabla 4.1 del material suplementario. Dentro de los datos obtenidos en fase exponencial de crecimiento se ha destacado la desregulación de tres grupos de genes. El primero incluye genes relacionados con el metabolismo de carbohidratos (principalmente genes relacionados con ruta de glicólisis), el segundo está constituido por genes de la familia de histonas y el último está formado por genes relacionados con respiración. En relación a este último grupo se ha señalado la subexpresión de los dos genes que codifican para componentes de la cadena de transportadora electrones. En relación con bajo nivel de poliaminas, se detectó la subexpresión de 3 genes que codifican para transportadores de azúcares, Tabla 4.1, HGT8, HGT6 y HGT7. C. albicans posee muchos genes (probablemente 19) que codifican aparentemente para transportadores de azúcares [44,124]. La mayoría de los genes HGT se expresan en $C$. albicans y la función de cada uno de ellos en el transporte de azucares ha sido demostrada [124,484]. La subexpresión de estos genes sugiere que las poliaminas intervienen en la regulación de los transportadores de azúcares en crecimiento de levadura. Además hemos detectado que la subexpresión de un número de genes implicados en ruta de glicólisis sugiere que las poliaminas también intervienen en la regulación de genes de glicólisis durante el crecimiento normal.

\section{Analisis del transcriptoma del mutante odc1/odc1 durante la transición levadura-hifa}

El mismo estudio de microarrays se llevó a cabo durante la transición levadurahifa. Este análisis mostró 1981 genes alterados en bajas concentraciones de poliaminas, con 599 genes de función desconocida. El nombre y la función de cada uno de ellos se han incluido en las Tablas 4.3 y 4.4 del material suplementario. En conjunto se detectó que la expresión de 1981 genes (1056 sobre- 925 sub-expresados) esta afectada por el cambio en las concentraciones de poliaminas, $0.01 \mathrm{mM}$ respecto a $10 \mathrm{mM}$. 503 genes son comunes a los tres tiempos ensayados (301 sobre- y 202 sub-expresados), 188 genes son comunes entre los 15 y 60 min (92 sobre- y 96 sub-expresados) y 263 genes entre los 60 y 180 min. (130 sobre- y 133 sub-expresados). 289 genes (124 sobre- y 165 subexpresados), 388 genes (196 sobre- y 192 sub-expresados), y 288 genes (181 sobre- y 107 sub-expresados) sólo varían en un tiempo de inducción (15, 60 y 180 
min. respectivamente). Considerando datos publicados por diversos autores sobre la modulación de la expresión génica durante la transición levadura-hifa, se trató de determinar qué genes implicados en morfogénesis se encontraban alterados en bajo concentraciones de poliaminas en la cepa Caodc1. Se han tenido en cuenta para ello datos de tres trabajos independientes publicados por Kadosh y Johnson [226], Lee et al [267] y Nantel et al [342]. Los 505 genes comunes a los tres tiempos se muestran en la Tabla 4.2. entre ellos se ha detectado, la subexpresión de varios genes que habían sido previamente reconocidos como hifa-específicos: ECE1 [32], RBT1 [37], HWP1 [419], DDR48 [261], PHR1 [370], RBT4 [37], HYR1 [370] y ALS3. Las poliaminas parecen actuar sobre la expresión de genes específicos de hifas de $C$. albicans. En los mutantes simple gcn5 y doble gcn5, hda1 cinco de ellos, ECE1, RBT1, HWP1, PHR1, HYR1 y ALS3, se encuentran subexpresados respecto a la cepa CAI4. Además, Nantel et al [342] mostró que la expresión de estos genes incrementa durante la transición levadura-hifa, lo cual es consistente con los resultados obtenidos para la cepa CAl4, por lo que podrían estar relacionados con la regulación de la morfogénesis, lo que confirma nuestros datos. Como se ha señalado anteriormente en el capitulo IV, se ha detectado la subexpresion de un número de genes relacionados con la pared celular incluidos AAF1, BGL22, CHS5, DPM1, ECM33, FGR6, GLC3, IPP1, PGA26, PGA36, PGA48, PGA53, PGA54, PGA59 y SUN41. Seis de estos genes BGL22, ECM33, FGR6, PGA54, PGA59 y SUN41 han sido identificados como sobrexpresados en la cepa CAI4 durante la transición levadura hifa (capitulo II). Entre los genes sobrexpresados se encuentran 6 genes, ECM1, MNN11, MNN4, PGA31, PHR2 y PIR1 involucrados en la pared celular a lo largo de la inducción. PIR1 cuya expresión disminuye e incrementa a largo de la indución en CAI4 y los mutantes gcn5 y gcn5, hda1 respectivamente. Estos resultados indican que la proteína Gcn5p y las poliaminas juegan, por tanto, un papel clave en la expresión de genes de pared. Otros grupos de genes subexpresados durante tres tiempos de inducción son: 4 genes que codifican para acetil-coenzimas ACC1, ACS1, ACS2 y $P O T 13,2$ genes implicada a la biosíntesis de adenina $A D E 13$ y $A D E 17$, seis genes de permeasas de aminoácidos AGP1, CAN2, CAN5, HNM3, IPF4580 y MAL31, 15 genes relacionados con el establecimiento de la polaridad ej. ACT1, ARF3, ARP3, CDC12 y COF1, 20 genes relacionados con la ruta de glicólisis y 12 genes relacionados con respiración y transporte de electrones (Tablas 4.3-4 material suplementario y Tabla 4.2).

CaCDC12 codifica para una septina esencial implicada en la formación de septos y tubos germinativos [497]. El homólogo del gen CaARF3 en S. cerevisiae forma 
parte de la superfamilia de genes RAS implicados en el desarrollo de la polaridad $[207,268]$. El homólogo del gen CaARP3 en S. cerevisiae es un componente esencial del complejo Arp2/3, involucrado en endocitosis y polaridad [102,464,478,510]. COF1 promueve la despolarización del filamento de actina de un modo dependiente del $\mathrm{pH}$. No se han encontrado alteraciones de estos genes relacionados con la polaridad en las mutantes como gcn5 y gcn5 hda1, lo que sugiere que las poliaminas están relacionadas con la regulación de estos genes; controlando de este modo el desarrollo de la transición de levadura-hifa.

Como se ha señalado anteriormente en el capitulo IV, en el mutante nulo Caodc1 bajo concentración de poliaminas $(0.01 \mathrm{mM})$ se ha detectado la subexpresion de un número significativo de genes relacionados los cuales intervienen en respiración y transporte de electrones, codificados en DNA genómico y DNA mitocondrial (COX13, CaNDH1, COX3A, COX3B, COX4, NAD6, CUP5, VAM6, STF2, PMA1 y VMA1, ATP20, ATP4, ATP7, COB ,COQ2, COX15, CTM1, CYT1, IPF12564, NAD1, NCP1, RIP1, TFP1, TFP3, VMA4, VMA7, VMA8, ATP14, ATP16, ATP2, ATP3, ATP5, ATP8, COX5A, CYT12, IPF3358, MCR1, QCR6 QCR9 у VMA2) durante el desarrollo de la transición de levadura-hifa. De acuerdo disminución de la actividad mitocondrial, ruta de glicólisis y ciclo de TCA, esto resulta en una menor producción de ATP. En la cepa delecionada se detectó subexpresión de genes relacionados con obtención de energía y transporte de electrones. Dentro de este grupo se encuentra la mayor parte de genes de la ruta de glicólisis y gluconeogénesis, además de genes implicados en otras rutas de obtención de energía. A bajas concentraciones de poliaminas aparecen subexpresados, en la cepa Caodc1, numerosos genes implicados en respiración, reflejando la mayor activación de este proceso en las hifas a altas concentraciones de poliaminas $(10 \mathrm{mM})$. En otros estudios hemos observado la subexpresión un número genes relacionados con la ruta de glicólisis en los mutantes gcn5 y gcn5, hda1 durante la transición levadura-hifa. Se ha descrito que la expresión de genes implicados en glicólisis aumenta durante la transición dimórfica en el mutante hda1 [307]. Estos cambios reflejan diferencias en la modulación de genes de metabolismo durante el desarrollo de pseudohifas e hifas. La regulación de la expresión de genes de glicólisis se ha asociado en diferentes trabajos a la transición dimórfica. Observándose que en el inicio de la transición levadura-hifa la expresión de genes que codifican enzimas de la ruta de glicólisis se reduce 
drásticamente y a continuación aumenta progresivamente, llegando a ser mayor en algunos genes, en hifas que en levaduras. Sin embargo, su relación con la morfogénesis no ha sido esclarecida, incluso se ha sugerido que se debe a las variaciones fisiológicas subyacentes al cambio en las condiciones de crecimiento y no al proceso morfogenético en sí [460]. Estos resultados indican que las poliaminas juegan un papel clave en la regulación de los genes necesarios para la respiración, lo que impide el desarrollo de las hifas de $C$. albicans.

Dentro de los grupos de genes subexpresados, se encuentran principalmente genes que codifican histonas (HHF21, HHF22, HHT21, HHT3, HTA1, HTA3 y HTB1) a 60 y 180 min. La subexpresión de estos genes podría estar relacionada en esta condición $(0.01 \mathrm{mM})$ con el retraso de crecimiento que muestra esta cepa respecto a la concentración de $10 \mathrm{mM}$ de putrescina, como se ha sugerido en otros mutantes donde estos genes se encuentran subexpresados y muestran crecimiento más lento [28]. A los 15 y 60 min de inducción, se detecta la subexpresión de genes implicados en la ruta de secreción como ARF21, CLC1, COP1, ERD2, ERV25, ERV46, SEC16, SEC18, SEC23, SEC26, SEC31 genes implicados en glicosilación como PMT2, PMT1, PMT4 y genes relacionados con mitosis y ciclo celular CDC1, CDC11, CDC42 y RAS1. CLC1 codifica para una proteína similar de S. cerevisiae Clc1p implicada en el transporte intracelular [425]. Ras1p actúa en el inicio de las rutas de transducción de señales PKA-cAMP y MAPK $[128,279,299]$. Estos resultados indican que las poliaminas parecen actuar sobre la expresión de genes implicados en diferentes aspectos de control del ciclo celular y ruta de secreción de C. albicans. Entre los grupos de genes sobreexpresados a lo largo de la inducción, destacan los genes asociados a pared celular incluidos BMS1, ECM1, MNN11, MNN4, PGA31, PHR2 y PIR1, genes que codifican para proteínas involucradas en el DNA-directed RNA polimerasa como RPB5, RPB8, RPC25, RPC40, RPC53, RPC82 y PAF1, genes asociados a procesamiento de rRNA (22 genes), RNA helicasa (11 genes) y factores transcripcionales (17 genes). Se observa la subrexpresión de genes que codifican proteínas ribosomales. En conjunto estos datos demuestran que la ausencia de poliaminas impiden la correcta activación de genes involucrados en el inicio de la filamentación, ruta de secreción, mitosis y ciclo celular, genes que codifican las histonas, genes que intervienen en respiración y transporte de electrones, genes relacionados con la ruta de glicólisis y metabolismo de carbohidratos y genes 
relacionados con la pared celular.

\section{Deleción del gene HAT1 en el mutante gcn5 de la cepa de RDG4}

La interrupción de los dos alelos del gen CaHAT1 en la cepa RDG4 (con el gen GCN5 delecionado) se llevó a cabo siguiendo la estrategia del "Ura-blaster" de forma similar a la descrita para la deleción del gen CaGCN5 en la cepa NDH4. Se partió para ello del cassette de deleción construido para la interrupción del gen CaHAT1 en la cepa CAI4 [106]. Se trata de un fragmento de $5.1 \mathrm{~kb}$ incluido en el vector pJBR3 que se corresponde con la secuencia hisG-CaURA3-hisG flanqueada por dos fragmentos de DNA, de 620 pb y 588 pb, de igual secuencia que el extremo $5^{\prime}$ de la ORF de CaHAT1 y la región $3^{\prime}$ no codificante del gen, respectivamente. El cassette completo de $5.1 \mathrm{~kb}$ fue liberado mediante la digestión del plásmido pJBR3 con las enzimas de restricción Sacl y Kpnl y utilizado para la transformación de la cepa RDG4. En la Figura 5.1 se muestra la comprobación del genotipo de las cepas generadas mediante experimentos tipo Southern. EI DNA genómico fue digerido con Sspl y como sonda se utilizó la secuencia de 588 pb correspondiente a la región 3' no codificante del gen CaHAT1. Tal y como puede apreciarse en la Figura 5.1, las cepas CAI4, RDG4 y heterozigotas para la deleción del gen presentan la banda de $0.64 \mathrm{~kb}$ correspondiente al alelo silvestre de CaHAT1. Esta banda no aparece en las cepas homozigotas para la deleción de CaHAT1 y en su lugar se detectan otras bandas cuyo tamaño se ajusta a la secuencia del cassette de deleción y de las secuencias flanqueantes. Nuestro interés se centra en la regulación de procesos morfogenéticos, por lo que se analizó principalmente la capacidad de las cepas gcn5, hat1 de llevar a cabo cambios morfológicos en respuesta a las diferentes condiciones del medio. En cada situación se comparó la morfología del doble mutante con la mostrada por la cepa silvestre y los mutantes sencillos gcn5 y hat1. En primer lugar se analizó la morfología celular y la velocidad de crecimiento a $28^{\circ} \mathrm{C}$ de la cepa con la doble deleción gcn5, hat1 (AHM4). En la Figura 5.2 y 5.3 se pueden apreciar claramente como el doble mutante AHM4 (gcn5, hat1), al igual que el mutante simple RDG4 (gcn5), presenta una morfología celular más agrumada que la cepa CAl4 y forma ramificaciones de células que se mantienen unidas y que acaban formando "rosetas". Mediante el seguimiento de la D.O. alcanzada a lo largo del tiempo por cultivos de las diferentes cepas, se detectó un ligero retraso en el crecimiento del doble mutante gcn5, hat1 y del mutante sencillo hat1 respecto a la cepa parental CAl4. Se estimó que entrada en fase exponencial de las cepas AHM4 (16 h) era 
mayor al de la cepa parental CAl4 y menor de la cepa RDG4 (gcn5). En relación con este fenotipo, en $S$. cerevisiae se ha descrito que la cepa diploide homozigota para la deleción del gen ScGCN5 muestra también defectos de crecimiento [461].

\section{Efecto de la doble deleción gcn5, hat1 en la transición dimórfica en medio líquido}

Después de inducir la transición dimórfica en medio líquido Lee [271] a $37^{\circ} \mathrm{C}$ se observó que el doble mutante forma pseudohifas en lugar de hifas (Figura 5.5), característica fenotípica descrita previamente en el mutante sencill Cagcn5 [106]. Un resultado similar se obtuvo en medio Lee suplementado con suero.

\section{Efecto de la doble deleción en la filamentación en medios sólidos}

Siguiendo con nuestra dinámica de análisis del fenotipo se estudió la capacidad del doble mutante de filamentar en medios sólidos, comparándola con la de los mutantes sencillos. El deleción de GCN5 causa defectos en la capacidad de filamentar en medio sólido Spider y medio Lee con NAcGlc, donde la miceliación se induce por la fuente de carbono. En la Figura 5.4 puede observarse como las cepas con la doble deleción gcn5, hat1 al igual que la mutante sencillo gcn5 presenta también defectos de filamentación en estos medios. Resulta más interesante analizar el efecto de la deleción en el medio embedded donde el mutante sencillo presenta un fenotipo diferente. El mutante simple RGL4 (hat1) a diferencia de RDG4 (gcn5), es capaz de desarrollar cierta filamentación. La cepa con la doble deleción presenta miceliación en este condicion, mostrando un fenotipo más próximo al mutante simple hat1 que al de gcn5

Efecto de la doble deleción gcn5, hat1 en la formación de las clamidosporas

La inducción de la formación de clamidosporas se llevó a cabo de forma análoga a la descrita en el análisis del mutante doble gcn5, hda1. En el mutante doble, así como en el mutante simple gcn5 no se detectaron clamidosporas, ni siquiera después de un periodo de incubación prolongado (14 días). Las células de la cepa hat1 fueron capaces de producir clamidosporas, demostrando con ello que el fenotipo observado se debe a la ausencia de GCN5. Se ha reportado, el requerimiento los procesos de remodelación de la cromatina por la formación de clamidosporas mediante la proteína Isw2p [353]. Otro gen cuya alteración causa defectos en la diferenciación clamidosporas es SCH9, que codifica una MAP 
quinasa que interviene en la regulación de diferentes procesos, entre los que se encuentra el almacenamiento de glucógeno, lo que está en concordancia con la función de las clamidosporas como estructuras de reserva [353]. Sería interesante por tanto determinar si la función de Gcn5p en el metabolismo de este polisacárido se encuentra conservada en $C$. albicans, de forma que pudieran relacionarse los defectos de acumulación de glucógeno con la incapacidad de formar clamidosporas. Se ha sugerido que la existencia en el genoma de C. albicans de numerosos genes sin homólogo conocido en otros organismos podría estar relacionada con el desarrollo de este proceso casi específico de C. albicans. Son necesarios por tanto nuevos trabajos sobre los mecanismos reguladores que desencadenan la formación de clamidosporas.

Análisis de la sensibilidad de la doble deleción gcn5, hat1 a compuestos que afectan a la pared celular

Por ultimo, decidimos analizar la sensibilidad a agentes que afectan a la pared celular: Cafeína, Calcofluor white, $\mathrm{NaCl}$, Higromicina $(100 \mu \mathrm{g} / \mathrm{ml})$, Itraconazol, Fluconazol (4 $\mu \mathrm{g} / \mathrm{ml})$, SDS y Rojo congo. Para cada uno de ellos se eligó la concentración máxima que no alteraba la vialidad de la cepa CAl4. Se crecieron las células hasta fase exponencial en medio YEPD y se sembraron distintas diluciones de las mismas en placas que contenían los diferentes compuestos. En la Figura 5.9 puede observarse como la cepa doble delecionada AHM4 muestra mayor sensibilidad a Higromycina, Fluconazole, Itraconazole y Caffeina. Este efecto se observó también en el doble mutante gcn5, hda1 y del mutante sencillo gcn5 respecto a la cepa parental CAI4. La cepa doble delecionada no reveló diferencias respecto a la cepa parental CAI4 a SDS y $\mathrm{NaCl}$.

Principales grupos funcionales de genes expresados diferencialmente a $28^{\circ} \mathrm{C}$ en el mutante gcn5, hat1

Durante el crecimiento como levaduras en medio mínimo YNB a $28^{\circ} \mathrm{C}$, en el mutante gcn5, hat1 se encuentra alterada la expresión de 521 genes, de los cuales, 247 genes están sobreexpresados y 274 subexpresados, con respecto a la cepa parental CAI4. Dentro del grupo de genes sobreexpresados en la cepa doble delecionada, las categorías y subcategorías estadísticamente significativas son respuesta a estrés oxidativo, metabolismo de carbohidratos, transporte a azúcar y metabolismo de amino ácidos. Dentro del grupo de genes subeexpresados en la cepa doble delecionada, las categorías y subcategorías estadísticamente 
significativas son, obtención de energía (principalmente a través de la glicólisis), metabolismo de carbohidratos, transporte de vitaminas y metabolismo de metionina. 


\section{Conclusiones}

1. Se llevó a cabo el análisis del transcriptoma de la cepa CAl4 durante la transición levadura hifa comparando con células crecidas en forma de levadura. EI cambio de temperatura altera la expresión de al menos 783 genes, considerando un factor de variación $\geq 1.5$ veces y hemos observado que:

2. La mayor parte de los genes expresado diferencialmente y los mayores niveles de variación se observaron a los 60 minutos, después las diferencias en la expresión génica desminuyen. Este resultado indica que la mayoría de las señales y genes reguladores de morfogénesis ejercen su función rápidamente y posteriormente sólo se expresan genes estructurales y de metabolismo.

3. Durante la transición dimórfica, aparece alterada la expresión de 51 genes correspondientes a proteínas de pared celular. La principal categoría asociada a genes relacionados a la familia de ALS, glucanasas, glucosidasas y manosiltransferasas y proteínas de unión mediante GPI.

4. Se ha llevado a cabo la deleción del gen GCN5 en la cepa NDH4 (hda1). La ausencia de ambas proteínas tiene un efecto en morfogénesis, ya que el doble mutante gcn5, hda1 presentó la mayoría los defectos acusados por el mutante sencillo gcn5: morfología unicamente de pseudohifas cuando crecen a $28^{\circ} \mathrm{C}$, defectos de filamentación, formación de pseudohifas en lugar de hifas en medios líquidos de inducción e incapacidad para formar clamidosporas.

5. El mutante gcn5, hda1 como en el mutante simple gcn5 muestra sensibilidad a $\mathrm{NaCl}$, Rojo congo, SDS, Calcoflour white, Fluconazol, Higromicina, Anfotericina y Cafeína.

6. Se ha comparado el transcriptoma del mutante doble gcn5, hda1 con el de la cepa parental CAI4, observándose que:

En fase exponencial de crecimiento a $28^{\circ} \mathrm{C}$, en la cepa delecionada, se encuentra alterada la expresión de 180 genes, considerando un factor de variación $\geq 1.5$ veces. Entre estos genes, aparecen subexpresados genes implicados en ruta de glicólisis y genes implicados en la biosíntesis de ribosomas lo que parece tener relación con el retraso en el crecimiento de la cepa doble delacionada.

Durante la transición dimórfica, aparece alterada la expresión de 664 genes. Las principales categorías asociadas a sobreexpresión son metabolismo de aminoácidos, pared celular, factores de transcripción incluyendo el gen que 
codifica el activador transcripcional Gcn4p. La subexpresión de genes que codifican para proteínas ribosomales y otros factores relacionados con funciones generales de crecimiento, refleja una respuesta en el mutante gcn5, hda1 a retraso en el crecimiento.

7. Se ha observado que el nivel de expresión de los genes relacionados con pared celular como ALS1, ALS3, ECE1, HWP1, PMT2, PMT4, MNT1, MNT2, MNT3 y SUN41 fue disminuyendo a la largo de transición levadura hifa en los mutantes simples gcn5, hda1 y en el mutante doble gcn5, hda1, lo que sugiere que estos genes están regulados por separado por ambos genes. El nivel de expresión de los genes PGA4, ECM2 y BGL2 aumenta en el mutante simple hda1 y disminuye en los mutantes gcn5 y doble gcn5, hda1 que es coherente con el fenotipo dominante de gcn5 en el mutante doble.

8. Se ha observado que el nivel de expresión de los genes relacionados con pared celular como RHD3, RBT5, PGA10, PGA31, PGA62 y YWP1 aumentan en ambos mutantes simple y doble durante transición levadura hifa indicando que estos genes se regulan independientemente por ambos genes. La expresión de los genes KRE1, CRH11, PHR1 y PGA59 aumenta en los mutantes simples gcn5 y doble gcn5, hda1 y disminuye en el mutante simple hda1. Además se ha observado que la expresión de los genes PHR1, CHR11, KRE1 y PGA59 aumenta en la cepa CAI4 durante la transición levadura hifa por cambio de temperatura. Estos resultados indican que HDA1 controla la expresión de estos genes.

9. Se ha llevado a cabo el análisis del transcriptoma del mutante odc1/odc1 durante el crecimiento en forma de levadura y durante la transición levadura hifa a concentraciones bajas de poliaminas $(0,01 \mathrm{mM}$ de putrescina) comparando con células crecidas en las mismas condiciones en altas concentraciones de poliaminas (10 $\mathrm{mM}$ de putrescina). El cambio de concentración de las poliaminas en el mutante odc1/odc1 altera la expresión de al menos 2118 genes, considerando un factor de variación $\geq 1.5$ veces.

10. En fase exponencial de crecimiento a $28^{\circ} \mathrm{C}$, se encuentra alterada la expresión de 137 genes, considerando un factor de variación $\geq 1.5$ veces. Entre estos genes, aparecen subexpresados genes implicados en transporte de electrones, pared celular, transporte de drogas y azúcares, factores de transcripción y genes implicados en la ruta de glicólisis que sugerimos están relacionados con el retraso en el crecimiento.

11. Durante la transición dimórfica, aparece alterada la expresión de 1981 genes. La principal categorías asociadas a sobreexpresión son genes que codifican para proteínas ribosomicas, pared celular, factores de transcripción, RNA helicasas y 
factores de inicio de transcripción.

12. Se ha observado que el nivel de expresión de los genes relacionados con el transporte de electrones y obtención de energía genes relacionados con ruta de glicólisis y ciclo de TCA disminuyen a largo de la transición levadura hifa. Además, hemos detectado subexpresion de genes relacionados con la ruta de secreción, polaridad celular, ciclo de desarrollo celular y transdución de señales que no se observó ni en los mutantes simples gcn5, hda1 el mutante doble gcn5, hda1 y ni en la cepa CAI4 durante transición levadura hifa.

13. Se ha llevado a cabo la deleción del gen HAT1 en la cepa RDG4 (gcn5). La ausencia de ambas proteínas tiene un efecto intermedio en morfogénesis, ya que el doble mutante gcn5, hat1 favorece la formación de pseudohifas en lugar de hifas, así como la incapacidad para formar clamidosporas como en el mutante simple gen5.

14. Se llevó a cabo del análisis el transcriptoma del mutante doble gcn5, hat1 durante el crecimiento en forma de levadura. Se observó que la deleción de los genes GCN5 y HAT1 altera la expresión de al menos 521 genes, considerando un factor de variación $\geq 1.5$ veces.

15. Entre los genes sobreexpresados se identificaron genes relacionados con pared celular, estrés oxidativo, oxidas/peroxidasas y transporte de azúcares. Se detectó la subexpresión de genes relacionados con la ruta de glicólisis, metabolismo de aminoácidos y de ácidos grasos. 


\section{Reference list}

1. Agalioti T, Lomvardas S, Parekh B, Yie J, Maniatis T, Thanos D (2000) Ordered recruitment of chromatin modifying and general transcription factors to the IFN-beta promoter. Cell 103:667-678.

2. Al-Hedaithy SS, Fotedar R (2002) Recovery and studies on chlamydosporenegative Candida albicans isolated from clinical specimens. Med Mycol 40:301306.

3. Alarco AM, Balan I, Talibi D, Mainville N, Raymond M (1997) AP1-mediated multidrug resistance in Saccharomyces cerevisiae requires FLR1 encoding a transporter of the major facilitator superfamily. J Biol Chem 272:19304-19313.

4. Alarco AM, Raymond M (1999) The bZip transcription factor Cap1p is involved in multidrug resistance and oxidative stress response in Candida albicans. J Bacteriol 181:700-708.

5. Albeck S, Dym O, Unger T, Snapir Z, Bercovich Z, Kahana C (2008) Crystallographic and biochemical studies revealing the structural basis for antizyme inhibitor function. Protein Sci 17:793-802.

6. Alberti-Segui C, Morales AJ, Xing H, Kessler MM, Willins DA, Weinstock KG, Cottarel G, Fechtel K, Rogers B (2004) Identification of potential cell-surface proteins in Candida albicans and investigation of the role of a putative cell-surface glycosidase in adhesion and virulence. Yeast 21:285-302.

7. Alter O, Brown PO, Botstein D (2003) Generalized singular value decomposition for comparative analysis of genome-scale expression data sets of two different organisms. Proc Natl Acad Sci U S A 100:3351-3356.

8. Allard S, Utley RT, Savard J, Clarke A, Grant P, Brandl CJ, Pillus L, Workman JL, Cote J (1999) NuA4, an essential transcription adaptor/histone H4 acetyltransferase complex containing Esa1p and the ATM-related cofactor Tra1p. Embo J 18:5108-5119.

9. Allfrey VG, Faulkner R, Mirsky AE (1964) Acetylation and Methylation of Histones and Their Possible Role in the Regulation of RNA Synthesis. Proc Natl Acad Sci U S A 51:786-794.

10. Allfrey VG, Littau VC, Mirsky AE (1963) On the role of of histones in regulation ribonucleic acid synthesis in the cell nucleus. Proc Natl Acad Sci U S A 49:414-421.

11. Allis CD, Chicoine LG, Richman R, Schulman IG (1985) Deposition-related histone acetylation in micronuclei of conjugating Tetrahymena. Proc Natl Acad Sci U S A 82:8048-8052.

12. Anderson JM, Soll DR (1986) Differences in actin localization during bud and hypha formation in the yeast Candida albicans. J Gen Microbiol 132:2035-2047.

13. Angus-Hill ML, Dutnall RN, Tafrov ST, Sternglanz R, Ramakrishnan V (1999) Crystal structure of the histone acetyltransferase Hpa2: A tetrameric member of the Gcn5-related N-acetyltransferase superfamily. J Mol Biol 294:1311-1325.

14. Babbar N, Gerner EW (2003) Polyamines as modifiers of genetic risk factors in human intestinal cancers. Biochem Soc Trans 31:388-392.

15. Baek YU, Li M, Davis DA (2008) Candida albicans ferric reductases are differentially regulated in response to distinct forms of iron limitation by the Rim101 and CBF transcription factors. Eukaryot Cell 7:1168-1179.

16. Bahn YS, Molenda M, Staab JF, Lyman CA, Gordon LJ, Sundstrom P (2007) Genome-wide transcriptional profiling of the cyclic AMP-dependent signaling pathway during morphogenic transitions of Candida albicans. Eukaryot Cell 6:23762390.

17. Bahn YS, Sundstrom P (2001) CAP1, an adenylate cyclase-associated protein gene, regulates bud-hypha transitions, filamentous growth, and cyclic AMP levels and is required for virulence of Candida albicans. J Bacteriol 183:3211-3223.

18. Bailey DA, Feldmann PJ, Bovey M, Gow NA, Brown AJ (1996) The Candida albicans HYR1 gene, which is activated in response to hyphal development, belongs to a gene family encoding yeast cell wall proteins. J Bacteriol 178:53535360 . 
19. Baillie GS, Douglas LJ (2000) Matrix polymers of Candida biofilms and their possible role in biofilm resistance to antifungal agents. J Antimicrob Chemother 46:397-403.

20. Ballou CE (1990) Isolation, characterization, and properties of Saccharomyces cerevisiae mnn mutants with nonconditional protein glycosylation defects. Methods Enzymol 185:440-470.

21. Banerjee D, Martin N, Nandi S, Shukla S, Dominguez A, Mukhopadhyay G, Prasad R (2007) A genome-wide steroid response study of the major human fungal pathogen Candida albicans. Mycopathologia 164:1-17.

22. Banerjee M, Thompson DS, Lazzell A, Carlisle PL, Pierce C, Monteagudo C, Lopez-Ribot JL, Kadosh D (2008) UME6, a novel filament-specific regulator of Candida albicans hyphal extension and virulence. Mol Biol Cell 19:1354-1365.

23. Barelle CJ, Priest CL, Maccallum DM, Gow NA, Odds FC, Brown AJ (2006) Nichespecific regulation of central metabolic pathways in a fungal pathogen. Cell Microbiol 8:961-971.

24. Beckerman J, Chibana H, Turner J, Magee PT (2001) Single-copy IMH3 allele is sufficient to confer resistance to mycophenolic acid in Candida albicans and to mediate transformation of clinical Candida species. Infect Immun 69:108-114.

25. Belden WJ, Barlowe C (1996) Erv25p, a component of COPII-coated vesicles, forms a complex with Emp24p that is required for efficient endoplasmic reticulum to Golgi transport. J Biol Chem 271:26939-26946.

26. Bennett RJ, Johnson AD (2006) The role of nutrient regulation and the Gpa2 protein in the mating pheromone response of $C$. albicans. Mol Microbiol 62:100119.

27. Bennett RJ, Uhl MA, Miller MG, Johnson AD (2003) Identification and characterization of a Candida albicans mating pheromone. Mol Cell Biol 23:81898201.

28. Bensen ES, Filler SG, Berman J (2002) A forkhead transcription factor is important for true hyphal as well as yeast morphogenesis in Candida albicans. Eukaryot Cell 1:787-798.

29. Bensen ES, Martin SJ, Li M, Berman J, Davis DA (2004) Transcriptional profiling in Candida albicans reveals new adaptive responses to extracellular $\mathrm{pH}$ and functions for Rim101p. Mol Microbiol 54:1335-1351.

30. Bergmann S, Ihmels J, Barkai N (2004) Similarities and differences in genome-wide expression data of six organisms. PLoS Biol 2:E9.

31. Bernstein BE, Humphrey EL, Erlich RL, Schneider R, Bouman P, Liu JS, Kouzarides T, Schreiber SL (2002) Methylation of histone H3 Lys 4 in coding regions of active genes. Proc Natl Acad Sci U S A 99:8695-8700.

32. Birse CE, Irwin MY, Fonzi WA, Sypherd PS (1993) Cloning and characterization of ECE1, a gene expressed in association with cell elongation of the dimorphic pathogen Candida albicans. Infect Immun 61:3648-3655.

33. Biswas D, Takahata S, Stillman DJ (2008) Different genetic functions for the Rpd3(L) and Rpd3(S) complexes suggest competition between NuA4 and Rpd3(S). Mol Cell Biol 28:4445-4458.

34. Biswas S, Van Dijck P, Datta A (2007) Environmental sensing and signal transduction pathways regulating morphopathogenic determinants of Candida albicans. Microbiol Mol Biol Rev 71:348-376.

35. Blasco JL, Garcia-Sanchez MA, Ruiz-Herrera J, Eslava AP, Iturriaga EA (2002) A gene coding for ornithine decarboxylase (odcA) is differentially expressed during the Mucor circinelloides yeast-to-hypha transition. Res Microbiol 153:155-164.

36. Boone C, Sdicu A, Laroche M, Bussey H (1991) Isolation from Candida albicans of a functional homolog of the Saccharomyces cerevisiae KRE1 gene, which is involved in cell wall beta-glucan synthesis. J Bacteriol 173:6859-6864.

37. Braun BR, Head WS, Wang MX, Johnson AD (2000) Identification and characterization of TUP1-regulated genes in Candida albicans. Genetics 156:3144. 
38. Braun BR, Kadosh D, Johnson AD (2001) NRG1, a repressor of filamentous growth in C.albicans, is down-regulated during filament induction. Embo J 20:4753-4761.

39. Braun BR, van Het Hoog M, d'Enfert C, Martchenko M, Dungan J, Kuo A, Inglis DO, Uhl MA, Hogues H, Berriman M, Lorenz M, Levitin A, Oberholzer U, Bachewich C, Harcus D, Marcil A, Dignard D, louk T, Zito R, Frangeul L, Tekaia F, Rutherford K, Wang E, Munro CA, Bates S, Gow NA, Hoyer LL, Kohler G, Morschhauser J, Newport G, Znaidi S, Raymond M, Turcotte B, Sherlock G, Costanzo M, Ihmels J, Berman J, Sanglard D, Agabian N, Mitchell AP, Johnson AD, Whiteway M, Nantel A (2005) A human-curated annotation of the Candida albicans genome. PLoS Genet 1:36-57.

40. Brega E, Zufferey R, Mamoun CB (2004) Candida albicans Csy1p is a nutrient sensor important for activation of amino acid uptake and hyphal morphogenesis. Eukaryot Cell 3:135-143.

41. Brown AJ. (2005). Integration of metabolism with virulence in Candida albicans (Genomics, F., Ed.), Mycota XIII, Springer-Verlag, Heidelberg.

42. Brown AJ, Barelle CJ, Budge S, Duncan J, Harris S, Lee PR, Leng P, Macaskill S, Abdul Murad AM, Ramsdale M, Wiltshire C, Wishart JA, Gow NA (2000) Gene regulation during morphogenesis in Candida albicans. Contrib Microbiol 5:112-125.

43. Brown DH, Jr., Giusani AD, Chen X, Kumamoto CA (1999) Filamentous growth of Candida albicans in response to physical environmental cues and its regulation by the unique CZF1 gene. Mol Microbiol 34:651-662.

44. Brown V, Sexton JA, Johnston M (2006) A glucose sensor in Candida albicans. Eukaryot Cell 5:1726-1737.

45. Brownell JE, Zhou J, Ranalli T, Kobayashi R, Edmondson DG, Roth SY, Allis CD (1996) Tetrahymena histone acetyltransferase A: a homolog to yeast Gcn5p linking histone acetylation to gene activation. Cell 84:843-851.

46. Bruno VM, Kalachikov S, Subaran R, Nobile CJ, Kyratsous C, Mitchell AP (2006) Control of the $C$. albicans cell wall damage response by transcriptional regulator Cas5. PLoS Pathog 2:e21.

47. Budillon A, Di Gennaro E, Bruzzese F, Rocco M, Manzo G, Caraglia M (2007) Histone deacetylase inhibitors: a new wave of molecular targeted anticancer agents. Recent Patents Anticancer Drug Discov 2:119-134.

48. Buffo J, Herman MA, Soll DR (1984) A characterization of pH-regulated dimorphism in Candida albicans. Mycopathologia 85:21-30.

49. Bustin M, Catez F, Lim JH (2005) The dynamics of histone $\mathrm{H} 1$ function in chromatin. Mol Cell 17:617-620.

50. Byun JA, Choi MH, Moon MH, Kong G, Chul Chung B (2009) Serum polyamines in pre- and post-operative patients with breast cancer corrected by menopausal status. Cancer Lett 273:300-304.

51. Calderone RA. (2002). Candida and Candidiasis, American Society for Microbiology, Washington, D.C.

53. Calderone RA, Fonzi WA (2001) Virulence factors of Candida albicans. Trends Microbiol 9:327-335.

54. Cao F, Lane S, Raniga PP, Lu Y, Zhou Z, Ramon K, Chen J, Liu H (2006) The Flo8 transcription factor is essential for hyphal development and virulence in Candida albicans. Mol Biol Cell 17:295-307.

55. Carmen AA, Griffin PR, Calaycay JR, Rundlett SE, Suka Y, Grunstein M (1999) Yeast HOS3 forms a novel trichostatin A-insensitive homodimer with intrinsic histone deacetylase activity. Proc Natl Acad Sci U S A 96:12356-12361.

56. Carroll SB (2000) Endless forms: the evolution of gene regulation and morphological diversity. Cell 101:577-580.

57. Carrozza MJ, Utley RT, Workman JL, Cote J (2003) The diverse functions of histone acetyltransferase complexes. Trends Genet 19:321-329.

58. Carruthers LM, Marton LJ, Peterson CL (2007) Polyamine analogues: potent inducers of nucleosomal array oligomerization and inhibitors of yeast cell growth. Biochem J 405:541-545. 
59. Casero RA, Jr., Marton LJ (2007) Targeting polyamine metabolism and function in cancer and other hyperproliferative diseases. Nat Rev Drug Discov 6:373-390.

60. Castilla R, Passeron S, Cantore ML (1998) N-acetyl-D-glucosamine induces germination in Candida albicans through a mechanism sensitive to inhibitors of cAMP-dependent protein kinase. Cell Signal 10:713-719.

61. Castillo L, Calvo E, Martinez Al, Ruiz-Herrera J, Valentin E, Lopez JA, Sentandreu R (2008) A study of the Candida albicans cell wall proteome. Proteomics 8:38713881.

62. Castillo L, Martinez Al, Garcera A, Garcia-Martinez J, Ruiz-Herrera J, Valentin E, Sentandreu R (2006) Genomic response programs of Candida albicans following protoplasting and regeneration. Fungal Genet Biol 43:124-134.

63. Celano P, Berchtold CM, Giardiello FM, Casero RA, Jr. (1989) Modulation of growth gene expression by selective alteration of polyamines in human colon carcinoma cells. Biochem Biophys Res Commun 165:384-390.

64. Ciurciu A, Komonyi O, Pankotai T, Boros IM (2006) The Drosophila histone acetyltransferase Gcn5 and transcriptional adaptor Ada2a are involved in nucleosomal histone H4 acetylation. Mol Cell Biol 26:9413-9423.

65. Cloutier M, Castilla R, Bolduc N, Zelada A, Martineau P, Bouillon M, Magee BB, Passeron S, Giasson L, Cantore ML (2003) The two isoforms of the cAMPdependent protein kinase catalytic subunit are involved in the control of dimorphism in the human fungal pathogen Candida albicans. Fungal Genet Biol 38:133-141.

66. Coffino P (2001) Antizyme, a mediator of ubiquitin-independent proteasomal degradation. Biochimie 83:319-323.

67. Coffino P (2001) Regulation of cellular polyamines by antizyme. Nat Rev Mol Cell Biol 2:188-194.

68. Cohen AE, Craven BM, Klooster WT (1997) Structure and thermal vibrations of spermine phosphate hexahydrate from neutron diffraction data at $125 \mathrm{~K}$. Acta Crystallogr B 53 ( Pt 5):787-794.

69. Cohen SS. (1998). A Guide To The Polyamines, Oxford University Press, Oxford.

70. Cole GT, Seshan KR, Phaneuf M, Lynn KT (1991) Chlamydospore-like cells of Candida albicans in the gastrointestinal tract of infected, immunocompromised mice. Can J Microbiol 37:637-646.

71. Coleman KG, Steensma HY, Kaback DB, Pringle JR (1986) Molecular cloning of chromosome I DNA from Saccharomyces cerevisiae: isolation and characterization of the CDC24 gene and adjacent regions of the chromosome. Mol Cell Biol 6:45164525.

72. Collins KA, Castillo AR, Tatsutani SY, Biggins S (2005) De novo kinetochore assembly requires the centromeric histone $\mathrm{H} 3$ variant. Mol Biol Cell 16:5649-5660.

73. Copping VM, Barelle CJ, Hube B, Gow NA, Brown AJ, Odds FC (2005) Exposure of Candida albicans to antifungal agents affects expression of SAP2 and SAP9 secreted proteinase genes. J Antimicrob Chemother 55:645-654.

74. Cornely OA (2008) Aspergillus to Zygomycetes: causes, risk factors, prevention, and treatment of invasive fungal infections. Infection 36:296-313.

75. Costanzo MC, Arnaud MB, Skrzypek MS, Binkley G, Lane C, Miyasato SR, Sherlock G (2006) The Candida Genome Database: facilitating research on Candida albicans molecular biology. FEMS Yeast Res 6:671-684.

76. Court H, Sudbery P (2007) Regulation of Cdc42 GTPase activity in the formation of hyphae in Candida albicans. Mol Biol Cell 18:265-281.

77. Cox GM, Mukherjee J, Cole GT, Casadevall A, Perfect JR (2000) Urease as a virulence factor in experimental cryptococcosis. Infect Immun 68:443-448.

78. Cuthbert GL, Daujat S, Snowden AW, Erdjument-Bromage H, Hagiwara T, Yamada M, Schneider R, Gregory PD, Tempst P, Bannister AJ, Kouzarides T (2004) Histone deimination antagonizes arginine methylation. Cell 118:545-553.

79. Cutler JE (1991) Putative virulence factors of Candida albicans. Annu Rev Microbiol 45:187-218.

80. Chabasse D, Bouchara JP, de Gentile L, Chennebault JM (1988) [Candida albicans 
chlamydospores observed in vivo in a patient with AIDS]. Ann Biol Clin (Paris) 46:817-818.

81. Chaffin WL, Lopez-Ribot JL, Casanova M, Gozalbo D, Martinez JP (1998) Cell wall and secreted proteins of Candida albicans: identification, function, and expression. Microbiol Mol Biol Rev 62:130-180.

82. Chandra J, Kuhn DM, Mukherjee PK, Hoyer LL, McCormick T, Ghannoum MA (2001) Biofilm formation by the fungal pathogen Candida albicans: development, architecture, and drug resistance. J Bacteriol 183:5385-5394.

83. Chandra J, Mukherjee PK, Leidich SD, Faddoul FF, Hoyer LL, Douglas LJ, Ghannoum MA (2001) Antifungal resistance of candidal biofilms formed on denture acrylic in vitro. J Dent Res 80:903-908.

84. Chang B, Chen Y, Zhao Y, Bruick RK (2007) JMJD6 is a histone arginine demethylase. Science 318:444-447.

85. Charron MJ, Dubin RA, Michels CA (1986) Structural and functional analysis of the MAL1 locus of Saccharomyces cerevisiae. Mol Cell Biol 6:3891-3899.

86. Chatterjee S, Mayor S (2001) The GPI-anchor and protein sorting. Cell Mol Life Sci 58:1969-1987.

87. Chattopadhyay MK, Murakami Y, Matsufuji S (2001) Antizyme regulates the degradation of ornithine decarboxylase in fission yeast Schizosaccharomyces pombe. Study in the spe2 knockout strains. J Biol Chem 276:21235-21241.

88. Chattopadhyay MK, Tabor CW, Tabor H (2005) Studies on the regulation of ornithine decarboxylase in yeast: effect of deletion in the MEU1 gene. Proc Natl Acad Sci U S A 102:16158-16163.

89. Chen D, Ma H, Hong H, Koh SS, Huang SM, Schurter BT, Aswad DW, Stallcup MR (1999) Regulation of transcription by a protein methyltransferase. Science 284:2174-2177.

90. Chen H, Tini M, Evans RM (2001) HATs on and beyond chromatin. Curr Opin Cell Biol 13:218-224.

91. Cheng G, Yeater KM, Hoyer LL (2006) Cellular and molecular biology of Candida albicans estrogen response. Eukaryot Cell 5:180-191.

92. Cheng S, Clancy CJ, Checkley MA, Handfield M, Hillman JD, Progulske-Fox A, Lewin AS, Fidel PL, Nguyen MH (2003) Identification of Candida albicans genes induced during thrush offers insight into pathogenesis. Mol Microbiol 48:1275-1288.

93. Cheung $P$, Allis CD, Sassone-Corsi $P$ (2000) Signaling to chromatin through histone modifications. Cell 103:263-271.

94. Childs AC, Mehta DJ, Gerner EW (2003) Polyamine-dependent gene expression. Cell Mol Life Sci 60:1394-1406.

95. Chrzanowska A, Krawczyk M, Baranczyk-Kuzma A (2008) Changes in arginase isoenzymes pattern in human hepatocellular carcinoma. Biochem Biophys Res Commun 377:337-340.

96. d'Enfert C, Goyard S, Rodriguez-Arnaveilhe S, Frangeul L, Jones L, Tekaia F, Bader O, Albrecht A, Castillo L, Dominguez A, Ernst JF, Fradin C, Gaillardin C, Garcia-Sanchez S, de Groot P, Hube B, Klis FM, Krishnamurthy S, Kunze D, Lopez MC, Mavor A, Martin N, Moszer I, Onesime D, Perez Martin J, Sentandreu R, Valentin E, Brown AJ (2005) CandidaDB: a genome database for Candida albicans pathogenomics. Nucleic Acids Res 33:D353-357.

97. Dabas N, Morschhauser J (2008) A transcription factor regulatory cascade controls secreted aspartic protease expression in Candida albicans. Mol Microbiol 69:586602.

98. David G, Dannenberg JH, Simpson N, Finnerty PM, Miao L, Turner GM, Ding Z, Carrasco R, Depinho RA (2006) Haploinsufficiency of the mSds3 chromatin regulator promotes chromosomal instability and cancer only upon complete neutralization of p53. Oncogene 25:7354-7360.

99. Davie JR (1998) Covalent modifications of histones: expression from chromatin templates. Curr Opin Genet Dev 8:173-178.

100. Davis RH, Hynes LV, Eversole-Cire P (1987) Nonsense mutations of the ornithine 
decarboxylase structural gene of Neurospora crassa. Mol Cell Biol 7:1122-1128.

101. Davis RH, Morris DR, Coffino P (1992) Sequestered end products and enzyme regulation: the case of ornithine decarboxylase. Microbiol Rev 56:280-290.

102. Dayel MJ, Holleran EA, Mullins RD (2001) Arp2/3 complex requires hydrolyzable ATP for nucleation of new actin filaments. Proc Natl Acad Sci U S A 98:1487114876.

103. De Bernardis F, Arancia S, Morelli L, Hube B, Sanglard D, Schafer W, Cassone A (1999) Evidence that members of the secretory aspartyl proteinase gene family, in particular SAP2, are virulence factors for Candida vaginitis. J Infect Dis 179:201208.

104. De Groot PW, Hellingwerf KJ, Klis FM (2003) Genome-wide identification of fungal GPI proteins. Yeast 20:781-796.

105. de Micheli M, Bille J, Schueller C, Sanglard D (2002) A common drug-responsive element mediates the upregulation of the Candida albicans ABC transporters CDR1 and $C D R 2$, two genes involved in antifungal drug resistance. Mol Microbiol 43:1197-1214.

106. Degano R. (in process). Acetilacion de Histonas en C. albicans. Alalisis de su function en regulacion de la expresion geneica y morfogenesis. Doctoral, Salamanca.

107. Denich KT, Malloy PJ, Feldman D (1992) Cloning and characterization of the gene encoding the ADP-ribosylation factor in Candida albicans. Gene 110:123-128.

108. Doedt T, Krishnamurthy S, Bockmuhl DP, Tebarth B, Stempel C, Russell CL, Brown AJ, Ernst JF (2004) APSES proteins regulate morphogenesis and metabolism in Candida albicans. Mol Biol Cell 15:3167-3180.

109. Douglas LJ (2003) Candida biofilms and their role in infection. Trends Microbiol 11:30-36.

110. Drobna E, Bialkova A, Subik J (2008) Transcriptional regulators of seven yeast species: comparative genome analysis. Review. Folia Microbiol (Praha) 53:275287.

111. Duden R (2003) ER-to-Golgi transport: COP I and COP II function (Review). Mol Membr Biol 20:197-207.

112. Duden R, Hosobuchi M, Hamamoto S, Winey M, Byers B, Schekman R (1994) Yeast beta- and beta'-coat proteins (COP). Two coatomer subunits essential for endoplasmic reticulum-to-Golgi protein traffic. J Biol Chem 269:24486-24495.

113. Dunkel N, Blass J, Rogers PD, Morschhauser J (2008) Mutations in the multi-drug resistance regulator MRR1, followed by loss of heterozygosity, are the main cause of MDR1 overexpression in fluconazole-resistant Candida albicans strains. Mol Microbiol 69:827-840.

114. Dunkel N, Liu TT, Barker KS, Homayouni R, Morschhauser J, Rogers PD (2008) A gain-of-function mutation in the transcription factor Upc2p causes upregulation of ergosterol biosynthesis genes and increased fluconazole resistance in a clinical Candida albicans isolate. Eukaryot Cell 7:1180-1190.

115. Dunkler A, Walther A, Specht CA, Wendland J (2005) Candida albicans CHT3 encodes the functional homolog of the Cts1 chitinase of Saccharomyces cerevisiae. Fungal Genet Biol 42:935-947.

116. Dupont B (2002) Overview of the lipid formulations of amphotericin B. J Antimicrob Chemother 49 Suppl 1:31-36.

117. Eberharter A, Sterner DE, Schieltz D, Hassan A, Yates JR, 3rd, Berger SL, Workman JL (1999) The ADA complex is a distinct histone acetyltransferase complex in Saccharomyces cerevisiae. Mol Cell Biol 19:6621-6631.

118. Ekwall K (2005) Genome-wide analysis of HDAC function. Trends Genet 21:608615.

119. Emre NC, Ingvarsdottir K, Wyce A, Wood A, Krogan NJ, Henry KW, Li K, Marmorstein R, Greenblatt JF, Shilatifard A, Berger SL (2005) Maintenance of low histone ubiquitylation by Ubp10 correlates with telomere-proximal Sir2 association and gene silencing. Mol Cell 17:585-594. 
120. Enjalbert B, Whiteway M (2005) Release from quorum-sensing molecules triggers hyphal formation during Candida albicans resumption of growth. Eukaryot Cell 4:1203-1210.

121. Ernst JF (2000) Regulation of dimorphism in Candida albicans. Contrib Microbiol 5:98-111.

122. Ernst JF (2000) Transcription factors in Candida albicans - environmental control of morphogenesis. Microbiology 146 ( Pt 8):1763-1774.

123. Fallon K, Bausch K, Noonan J, Huguenel E, Tamburini P (1997) Role of aspartic proteases in disseminated Candida albicans infection in mice. Infect Immun 65:551556.

124. Fan J, Chaturvedi V, Shen SH (2002) Identification and phylogenetic analysis of a glucose transporter gene family from the human pathogenic yeast Candida albicans. J Mol Evol 55:336-346.

125. Fan Q, An L, Cui L (2004) Plasmodium falciparum histone acetyltransferase, a yeast GCN5 homologue involved in chromatin remodeling. Eukaryot Cell 3:264276.

126. Fazzio TG, Kooperberg C, Goldmark JP, Neal C, Basom R, Delrow J, Tsukiyama T (2001) Widespread collaboration of Isw2 and Sin3-Rpd3 chromatin remodeling complexes in transcriptional repression. Mol Cell Biol 21:6450-6460.

127. Felk A, Kretschmar M, Albrecht A, Schaller M, Beinhauer S, Nichterlein T, Sanglard D, Korting HC, Schafer W, Hube B (2002) Candida albicans hyphal formation and the expression of the Efg1-regulated proteinases Sap4 to Sap6 are required for the invasion of parenchymal organs. Infect Immun 70:3689-3700.

128. Feng Q, Summers E, Guo B, Fink G (1999) Ras signaling is required for seruminduced hyphal differentiation in Candida albicans. J Bacteriol 181:6339-6346.

129. Fernandez-Arenas E, Cabezon V, Bermejo C, Arroyo J, Nombela C, Diez-Orejas R, Gil C (2007) Integrated proteomics and genomics strategies bring new insight into Candida albicans response upon macrophage interaction. Mol Cell Proteomics 6:460-478.

130. Firon A, Aubert S, Iraqui I, Guadagnini S, Goyard S, Prevost MC, Janbon G, d'Enfert C (2007) The SUN41 and SUN42 genes are essential for cell separation in Candida albicans. Mol Microbiol 66:1256-1275.

131. Fonzi WA (1999) PHR1 and PHR2 of Candida albicans encode putative glycosidases required for proper cross-linking of beta-1,3- and beta-1,6-glucans. J Bacteriol 181:7070-7079.

132. Fonzi WA, Irwin MY (1993) Isogenic strain construction and gene mapping in Candida albicans. Genetics 134:717-728.

133. Fonzi WA, Sypherd PS (1987) The gene and the primary structure of ornithine decarboxylase from Saccharomyces cerevisiae. J Biol Chem 262:10127-10133.

134. Fradin C, De Groot P, MacCallum D, Schaller M, Klis F, Odds FC, Hube B (2005) Granulocytes govern the transcriptional response, morphology and proliferation of Candida albicans in human blood. Mol Microbiol 56:397-415.

135. Fratti RA, Belanger PH, Sanati H, Ghannoum MA (1998) The effect of the new triazole, voriconazole (UK-109,496), on the interactions of Candida albicans and Candida krusei with endothelial cells. J Chemother 10:7-16.

136. Freitas MA, Sklenar AR, Parthun MR (2004) Application of mass spectrometry to the identification and quantification of histone post-translational modifications. J Cell Biochem 92:691-700.

137. Frydman B, Westler WM, Samejima K (1996) Spermine Binds in Solution to the TpsiC Loop of tRNA(Phe): Evidence from a $750 \mathrm{MHz}$ (1)H-NMR Analysis. J Org Chem 61:2588-2589.

138. Fu Y, Ibrahim AS, Sheppard DC, Chen YC, French SW, Cutler JE, Filler SG, Edwards JE, Jr. (2002) Candida albicans Als1p: an adhesin that is a downstream effector of the EFG1 filamentation pathway. Mol Microbiol 44:61-72.

139. Garcia-Sanchez S, Aubert S, Iraqui I, Janbon G, Ghigo JM, d'Enfert C (2004) Candida albicans biofilms: a developmental state associated with specific and 
stable gene expression patterns. Eukaryot Cell 3:536-545.

140. Garcia-Sanchez S, Mavor AL, Russell CL, Argimon S, Dennison P, Enjalbert B, Brown AJ (2005) Global roles of Ssn6 in Tup1- and Nrg1-dependent gene regulation in the fungal pathogen, Candida albicans. Mol Biol Cell 16:2913-2925.

141. Garcia MG, O'Connor JE, Garcia LL, Martinez SI, Herrero E, del Castillo Agudo L (2001) Isolation of a Candida albicans gene, tightly linked to URA3, coding for a putative transcription factor that suppresses a Saccharomyces cerevisiae aft1 mutation. Yeast 18:301-311.

142. Gasch AP, Spellman PT, Kao CM, Carmel-Harel O, Eisen MB, Storz G, Botstein D, Brown PO (2000) Genomic expression programs in the response of yeast cells to environmental changes. Mol Biol Cell 11:4241-4257.

143. Gaur M, Puri N, Manoharlal R, Rai V, Mukhopadhayay G, Choudhury D, Prasad R (2008) MFS transportome of the human pathogenic yeast Candida albicans. BMC Genomics 9:579.

144. Gavin AC, Bosche M, Krause R, Grandi P, Marzioch M, Bauer A, Schultz J, Rick JM, Michon AM, Cruciat CM, Remor M, Hofert C, Schelder M, Brajenovic M, Ruffner H, Merino A, Klein K, Hudak M, Dickson D, Rudi T, Gnau V, Bauch A, Bastuck S, Huhse B, Leutwein C, Heurtier MA, Copley RR, Edelmann A, Querfurth E, Rybin V, Drewes G, Raida M, Bouwmeester T, Bork P, Seraphin B, Kuster B, Neubauer G, Superti-Furga G (2002) Functional organization of the yeast proteome by systematic analysis of protein complexes. Nature 415:141-147.

145. Gerich B, Orci L, Tschochner H, Lottspeich F, Ravazzola M, Amherdt M, Wieland F, Harter C (1995) Non-clathrin-coat protein alpha is a conserved subunit of coatomer and in Saccharomyces cerevisiae is essential for growth. Proc Natl Acad Sci U S A 92:3229-3233.

146. Gerner EW, Meyskens FL, Jr. (2004) Polyamines and cancer: old molecules, new understanding. Nat Rev Cancer 4:781-792.

147. Ghannoum MA (2000) Potential role of phospholipases in virulence and fungal pathogenesis. Clin Microbiol Rev 13:122-143, table of contents.

148. Gilfillan GD, Sullivan DJ, Haynes K, Parkinson T, Coleman DC, Gow NA (1998) Candida dubliniensis: phylogeny and putative virulence factors. Microbiology 144 ( Pt 4):829-838.

149. Gillum AM, Tsay EY, Kirsch DR (1984) Isolation of the Candida albicans gene for orotidine-5'-phosphate decarboxylase by complementation of $S$. cerevisiae ura3 and E. coli pyrF mutations. Mol Gen Genet 198:179-182.

150. Gimeno RE, Espenshade P, Kaiser CA (1996) COPII coat subunit interactions: Sec24p and Sec23p bind to adjacent regions of Sec16p. Mol Biol Cell 7:1815-1823.

151. Giusani AD, Vinces M, Kumamoto CA (2002) Invasive filamentous growth of Candida albicans is promoted by Czf1p-dependent relief of Efg1p-mediated repression. Genetics 160:1749-1753.

152. Goldknopf IL, Busch H (1977) Isopeptide linkage between nonhistone and histone 2A polypeptides of chromosomal conjugate-protein A24. Proc Natl Acad Sci U S A 74:864-868.

153. Gompel N, Prud'homme B, Wittkopp PJ, Kassner VA, Carroll SB (2005) Chance caught on the wing: cis-regulatory evolution and the origin of pigment patterns in Drosophila. Nature 433:481-487.

154. Govind CK, Zhang F, Qiu H, Hofmeyer K, Hinnebusch AG (2007) Gcn5 promotes acetylation, eviction, and methylation of nucleosomes in transcribed coding regions. Mol Cell 25:31-42.

155. Gow NA, Brown AJ, Odds FC (2002) Fungal morphogenesis and host invasion. Curr Opin Microbiol 5:366-371.

156. Gow NA, Henderson G, Gooday GW (1986) Cytological interrelationships between the cell cycle and duplication cycle of Candida albicans. Microbios 47:97-105.

157. Gow NAR. (2002). Cell biology and cell cycle of Candida albicans In R.A. Calderone (ed.), Candida and Candidiasis, American Society for Microbiology, Washington, D.C. 
158. Goyard S, Knechtle P, Chauvel M, Mallet A, Prevost MC, Proux C, Coppee JY, Schwartz P, Dromer F, Park H, Filler SG, Janbon G, d'Enfert C (2008) The Yak1 kinase is involved in the initiation and maintenance of hyphal growth in Candida albicans. Mol Biol Cell 19:2251-2266.

159. Granger BL, Flenniken ML, Davis DA, Mitchell AP, Cutler JE (2005) Yeast wall protein 1 of Candida albicans. Microbiology 151:1631-1644.

160. Grant PA, Berger SL (1999) Histone acetyltransferase complexes. Semin Cell Dev Biol 10:169-177.

161. Grant PA, Berger SL, Workman JL (1999) Identification and analysis of native nucleosomal histone acetyltransferase complexes. Methods Mol Biol 119:311-317.

162. Grant PA, Duggan L, Cote J, Roberts SM, Brownell JE, Candau R, Ohba R, OwenHughes T, Allis CD, Winston F, Berger SL, Workman JL (1997) Yeast Gcn5 functions in two multisubunit complexes to acetylate nucleosomal histones: characterization of an Ada complex and the SAGA (Spt/Ada) complex. Genes Dev 11:1640-1650.

163. Grant PA, Schieltz D, Pray-Grant MG, Steger DJ, Reese JC, Yates JR, 3rd, Workman JL (1998) A subset of TAF(II)s are integral components of the SAGA complex required for nucleosome acetylation and transcriptional stimulation. Cell 94:45-53.

164. Green CB, Zhao X, Yeater KM, Hoyer LL (2005) Construction and real-time RTPCR validation of Candida albicans PALS-GFP reporter strains and their use in flow cytometry analysis of ALS gene expression in budding and filamenting cells. Microbiology 151:1051-1060.

165. Green SR, Johnson AD (2004) Promoter-dependent roles for the Srb10 cyclindependent kinase and the Hda1 deacetylase in Tup1-mediated repression in Saccharomyces cerevisiae. Mol Biol Cell 15:4191-4202.

166. Gregory PD, Schmid A, Zavari M, Lui L, Berger SL, Horz W (1998) Absence of Gcn5 HAT activity defines a novel state in the opening of chromatin at the PHO5 promoter in yeast. Mol Cell 1:495-505.

167. Grillo MA, Colombatto S (1994) Polyamine transport in cells. Biochem Soc Trans 22:894-898.

168. Gudlaugsson O, Gillespie S, Lee K, Vande Berg J, Hu J, Messer S, Herwaldt L, Pfaller M, Diekema D (2003) Attributable mortality of nosocomial candidemia, revisited. Clin Infect Dis 37:1172-1177.

169. Guevara-Olvera L, Hung CY, Yu JJ, Cole GT (2000) Sequence, expression and functional analysis of the Coccidioides immitis ODC (ornithine decarboxylase) gene. Gene 242:437-448.

170. Guevara-Olvera L, Xoconostle-Cazares B, Ruiz-Herrera J (1997) Cloning and disruption of the ornithine decarboxylase gene of Ustilago maydis: evidence for a role of polyamines in its dimorphic transition. Microbiology 143 ( Pt 7):2237-2245.

171. Gupta R, Hamasaki-Katagiri N, White Tabor C, Tabor H (2001) Effect of spermidine on the in vivo degradation of ornithine decarboxylase in Saccharomyces cerevisiae. Proc Natl Acad Sci U S A 98:10620-10623.

172. Guschin D, Wade PA, Kikyo N, Wolffe AP (2000) ATP-Dependent histone octamer mobilization and histone deacetylation mediated by the Mi-2 chromatin remodeling complex. Biochemistry 39:5238-5245.

173. Halme A, Bumgarner S, Styles C, Fink GR (2004) Genetic and epigenetic regulation of the FLO gene family generates cell-surface variation in yeast. Cell 116:405-415.

174. Han J, Zhou H, Horazdovsky B, Zhang K, Xu RM, Zhang Z (2007) Rtt109 acetylates histone $\mathrm{H} 3$ lysine 56 and functions in DNA replication. Science 315:653655.

175. Hanahan D, Meselson M (1983) Plasmid screening at high colony density. Methods Enzymol 100:333-342.

176. Hansen JC (2002) Conformational dynamics of the chromatin fiber in solution: determinants, mechanisms, and functions. Annu Rev Biophys Biomol Struct 
31:361-392.

177. Harcus D, Nantel A, Marcil A, Rigby T, Whiteway M (2004) Transcription profiling of cyclic AMP signaling in Candida albicans. Mol Biol Cell 15:4490-4499.

178. Hardwick KG, Lewis MJ, Semenza J, Dean N, Pelham HR (1990) ERD1, a yeast gene required for the retention of luminal endoplasmic reticulum proteins, affects glycoprotein processing in the Golgi apparatus. Embo J 9:623-630.

179. Hassan AH, Neely KE, Workman JL (2001) Histone acetyltransferase complexes stabilize swi/snf binding to promoter nucleosomes. Cell 104:817-827.

180. Hayashi S, Murakami Y, Matsufuji S (1996) Ornithine decarboxylase antizyme: a novel type of regulatory protein. Trends Biochem Sci 21:27-30.

181. Hayashi S, Murakami Y, Matsufuji S, Nishiyama M, Kanamoto R, Kameji T (1988) Studies on ornithine decarboxylase antizyme. Adv Exp Med Biol 250:25-35.

182. Hazan I, Sepulveda-Becerra M, Liu H (2002) Hyphal elongation is regulated independently of cell cycle in Candida albicans. Mol Biol Cell 13:134-145.

183. Hazen KC, Glee PM (1994) Hydrophobic cell wall protein glycosylation by the pathogenic fungus Candida albicans. Can J Microbiol 40:266-272.

184. He Y, Kashiwagi K, Fukuchi J, Terao K, Shirahata A, Igarashi K (1993) Correlation between the inhibition of cell growth by accumulated polyamines and the decrease of magnesium and ATP. Eur J Biochem 217:89-96.

185. He Y, Suzuki T, Kashiwagi K, Kusama-Eguchi K, Shirahata A, Igarashi K (1994) Correlation between the inhibition of cell growth by bis(ethyl)polyamine analogues and the decrease in the function of mitochondria. Eur J Biochem 221:391-398.

186. Heller JS, Fong WF, Canellakis ES (1976) Induction of a protein inhibitor to ornithine decarboxylase by the end products of its reaction. Proc Natl Acad Sci U S A 73:1858-1862.

187. Henry KW, Wyce A, Lo WS, Duggan LJ, Emre NC, Kao CF, Pillus L, Shilatifard A, Osley MA, Berger SL (2003) Transcriptional activation via sequential histone H2B ubiquitylation and deubiquitylation, mediated by SAGA-associated Ubp8. Genes Dev 17:2648-2663.

188. Herrero AB, Lopez MC, Garcia S, Schmidt A, Spaltmann F, Ruiz-Herrera J, Dominguez A (1999) Control of filament formation in Candida albicans by polyamine levels. Infect Immun 67:4870-4878.

189. Herrero AB, Uccelletti D, Hirschberg CB, Dominguez A, Abeijon C (2002) The Golgi GDPase of the fungal pathogen Candida albicans affects morphogenesis, glycosylation, and cell wall properties. Eukaryot Cell 1:420-431.

190. Higashi K, Kashiwagi K, Taniguchi S, Terui Y, Yamamoto K, Ishihama A, Igarashi K (2006) Enhancement of +1 frameshift by polyamines during translation of polypeptide release factor 2 in Escherichia coli. J Biol Chem 281:9527-9537.

191. Hiller E, Heine S, Brunner H, Rupp S (2007) Candida albicans Sun41p, a putative glycosidase, is involved in morphogenesis, cell wall biogenesis, and biofilm formation. Eukaryot Cell 6:2056-2065.

192. Hinnebusch AG, Fink GR (1983) Positive regulation in the general amino acid control of Saccharomyces cerevisiae. Proc Natl Acad Sci U S A 80:5374-5378.

193. Hlubek A, Schink KO, Mahlert M, Sandrock B, Bolker M (2008) Selective activation by the guanine nucleotide exchange factor Don1 is a main determinant of Cdc42 signalling specificity in Ustilago maydis. Mol Microbiol 68:615-623.

194. Hobbs CA, Gilmour SK (2000) High levels of intracellular polyamines promote histone acetyltransferase activity resulting in chromatin hyperacetylation. J Cell Biochem 77:345-360.

195. Hogarty MD, Norris MD, Davis K, Liu X, Evageliou NF, Hayes CS, Pawel B, Guo R, Zhao H, Sekyere E, Keating J, Thomas W, Cheng NC, Murray J, Smith J, Sutton R, Venn N, London WB, Buxton A, Gilmour SK, Marshall GM, Haber M (2008) ODC1 is a critical determinant of MYCN oncogenesis and a therapeutic target in neuroblastoma. Cancer Res 68:9735-9745.

196. Hogues H, Lavoie H, Sellam A, Mangos M, Roemer T, Purisima E, Nantel A, Whiteway M (2008) Transcription factor substitution during the evolution of fungal 
ribosome regulation. Mol Cell 29:552-562.

197. Holtta E, Hovi T (1985) Polyamine depletion results in impairment of polyribosome formation and protein synthesis before onset of DNA synthesis in mitogen-activated human lymphocytes. Eur J Biochem 152:229-237.

198. Hoppen J, Dietz M, Warsow G, Rohde R, Schuller HJ (2007) Ribosomal protein genes in the yeast Candida albicans may be activated by a heterodimeric transcription factor related to Ino2 and Ino4 from S. cerevisiae. Mol Genet Genomics 278:317-330.

199. Howe L, Auston D, Grant P, John S, Cook RG, Workman JL, Pillus L (2001) Histone H3 specific acetyltransferases are essential for cell cycle progression. Genes Dev 15:3144-3154.

200. Hoyer LL (2001) The ALS gene family of Candida albicans. Trends Microbiol 9:176180.

201. Hoyer LL, Green CB, Oh SH, Zhao X (2008) Discovering the secrets of the Candida albicans agglutinin-like sequence (ALS) gene family--a sticky pursuit. Med Mycol 46:1-15.

202. Hoyer LL, Hecht JE (2001) The ALS5 gene of Candida albicans and analysis of the Als5p N-terminal domain. Yeast 18:49-60.

203. Hoyer LL, Payne TL, Bell M, Myers AM, Scherer S (1998) Candida albicans ALS3 and insights into the nature of the ALS gene family. Curr Genet 33:451-459.

204. Hoyer LL, Payne TL, Hecht JE (1998) Identification of Candida albicans ALS2 and ALS4 and localization of als proteins to the fungal cell surface. J Bacteriol 180:5334-5343.

205. Hoyer LL, Scherer S, Shatzman AR, Livi GP (1995) Candida albicans ALS1: domains related to a Saccharomyces cerevisiae sexual agglutinin separated by a repeating motif. Mol Microbiol 15:39-54.

206. Hoyt MA, Broun M, Davis RH (2000) Polyamine regulation of ornithine decarboxylase synthesis in Neurospora crassa. Mol Cell Biol 20:2760-2773.

207. Huang CF, Liu YW, Tung L, Lin CH, Lee FJ (2003) Role for Arf3p in development of polarity, but not endocytosis, in Saccharomyces cerevisiae. Mol Biol Cell 14:38343847.

208. Huang $\mathrm{H}$, Harcus D, Whiteway M (2008) Transcript profiling of a MAP kinase pathway in C. albicans. Microbiol Res 163:380-393.

209. Hubbert C, Guardiola A, Shao R, Kawaguchi Y, Ito A, Nixon A, Yoshida M, Wang $\mathrm{XF}$, Yao TP (2002) HDAC6 is a microtubule-associated deacetylase. Nature 417:455-458.

210. Hube B (1996) Candida albicans secreted aspartyl proteinases. Curr Top Med Mycol 7:55-69.

211. Hube B, Monod M, Schofield DA, Brown AJ, Gow NA (1994) Expression of seven members of the gene family encoding secretory aspartyl proteinases in Candida albicans. Mol Microbiol 14:87-99.

212. Hudson DA, Sciascia QL, Sanders RJ, Norris GE, Edwards PJ, Sullivan PA, Farley PC (2004) Identification of the dialysable serum inducer of germ-tube formation in Candida albicans. Microbiology 150:3041-3049.

213. Hull CM, Johnson AD (1999) Identification of a mating type-like locus in the asexual pathogenic yeast Candida albicans. Science 285:1271-1275.

214. Hunt LT, Dayhoff MO (1977) Amino-terminal sequence identity of ubiquitin and the nonhistone component of nuclear protein A24. Biochem Biophys Res Commun 74:650-655.

215. Igarashi K, Kashiwagi K (2000) Polyamines: mysterious modulators of cellular functions. Biochem Biophys Res Commun 271:559-564.

216. Imoberdorf RM, Topalidou I, Strubin M (2006) A role for gcn5-mediated global histone acetylation in transcriptional regulation. Mol Cell Biol 26:1610-1616.

217. Ishihara N, Hamasaki M, Yokota S, Suzuki K, Kamada Y, Kihara A, Yoshimori T, Noda T, Ohsumi $Y$ (2001) Autophagosome requires specific early Sec proteins for its formation and NSF/SNARE for vacuolar fusion. Mol Biol Cell 12:3690-3702. 
218. Jain P, Akula I, Edlind T (2003) Cyclic AMP signaling pathway modulates susceptibility of candida species and Saccharomyces cerevisiae to antifungal azoles and other sterol biosynthesis inhibitors. Antimicrob Agents Chemother 47:3195-3201.

219. Jimenez-Bremont JF, Rodriguez-Kessler M, Rodriguez-Guerra R, Cortes-Penagos C, Torres-Guzman JC, Williamson JS (2006) Cloning and sequence analysis of ornithine decarboxylase gene fragments from the Ascomycota. DNA Seq 17:231236.

220. Jimenez-Bremont JF, Ruiz-Herrera J (2008) Analysis of the transcriptional regulation of YIODC gene from the dimorphic fungus Yarrowia lipolytica. Microbiol Res.

221. Jimenez-Bremont JF, Ruiz-Herrera J, Dominguez A (2001) Disruption of gene YIODC reveals absolute requirement of polyamines for mycelial development in Yarrowia lipolytica. FEMS Yeast Res 1:195-204.

222. Jin YH, Dunlap PE, McBride SJ, Al-Refai H, Bushel PR, Freedman JH (2008) Global transcriptome and deletome profiles of yeast exposed to transition metals. PLoS Genet 4:e1000053.

223. John S, Howe L, Tafrov ST, Grant PA, Sternglanz R, Workman JL (2000) The something about silencing protein, Sas3, is the catalytic subunit of NuA3, a yTAF(II)30-containing HAT complex that interacts with the Spt16 subunit of the yeast CP (Cdc68/Pob3)-FACT complex. Genes Dev 14:1196-1208.

224. Jones T, Federspiel NA, Chibana H, Dungan J, Kalman S, Magee BB, Newport G, Thorstenson YR, Agabian N, Magee PT, Davis RW, Scherer S (2004) The diploid genome sequence of Candida albicans. Proc Natl Acad Sci U S A 101:7329-7334.

225. Jung IL, Kim IG (2003) Transcription of ahpC, katG, and katE genes in Escherichia coli is regulated by polyamines: polyamine-deficient mutant sensitive to $\mathrm{H}_{2} \mathrm{O}_{2}$ induced oxidative damage. Biochem Biophys Res Commun 301:915-922.

226. Kadosh D, Johnson AD (2005) Induction of the Candida albicans filamentous growth program by relief of transcriptional repression: a genome-wide analysis. Mol Biol Cell 16:2903-2912.

227. Kadosh D, Johnson AD (2001) Rfg1, a protein related to the Saccharomyces cerevisiae hypoxic regulator Rox1, controls filamentous growth and virulence in Candida albicans. Mol Cell Biol 21:2496-2505.

228. Kaiser B, Munder T, Saluz HP, Kunkel W, Eck R (1999) Identification of a gene encoding the pyruvate decarboxylase gene regulator CaPdc2p from Candida albicans. Yeast 15:585-591.

229. Kaneko A, Umeyama T, Utena-Abe Y, Yamagoe S, Niimi M, Uehara Y (2006) Tcc1p, a novel protein containing the tetratricopeptide repeat motif, interacts with Tup1p to regulate morphological transition and virulence in Candida albicans. Eukaryot Cell 5:1894-1905.

230. Kao AS, Brandt ME, Pruitt WR, Conn LA, Perkins BA, Stephens DS, Baughman WS, Reingold AL, Rothrock GA, Pfaller MA, Pinner RW, Hajjeh RA (1999) The epidemiology of candidemia in two United States cities: results of a populationbased active surveillance. Clin Infect Dis 29:1164-1170.

231. Karababa M, Coste AT, Rognon B, Bille J, Sanglard D (2004) Comparison of gene expression profiles of Candida albicans azole-resistant clinical isolates and laboratory strains exposed to drugs inducing multidrug transporters. Antimicrob Agents Chemother 48:3064-3079.

232. Kelly MT, MacCallum DM, Clancy SD, Odds FC, Brown AJ, Butler G (2004) The Candida albicans CaACE2 gene affects morphogenesis, adherence and virulence. Mol Microbiol 53:969-983.

233. Kelly R, Kwon-Chung KJ (1992) A zinc finger protein from Candida albicans is involved in sucrose utilization. J Bacteriol 174:222-232.

234. Khochbin S (2001) Histone $\mathrm{H} 1$ diversity: bridging regulatory signals to linker histone function. Gene 271:1-12.

235. Kim S, Wolyniak MJ, Staab JF, Sundstrom P (2007) A 368-base-pair cis-acting 
HWP1 promoter region, HCR, of Candida albicans confers hypha-specific gene regulation and binds architectural transcription factors Nhp6 and Gcf1p. Eukaryot Cell 6:693-709.

236. Kimura A, Matsubara K, Horikoshi M (2005) A decade of histone acetylation: marking eukaryotic chromosomes with specific codes. J Biochem (Tokyo) 138:647662.

237. Kleff S, Andrulis ED, Anderson CW, Sternglanz R (1995) Identification of a gene encoding a yeast histone $\mathrm{H} 4$ acetyltransferase. J Biol Chem 270:24674-24677.

238. Kleinsmith LJ, Allfrey VG, Mirsky AE (1966) Phosphoprotein metabolism in isolated lymphocyte nuclei. Proc Natl Acad Sci U S A 55:1182-1189.

239. Klengel T, Liang WJ, Chaloupka J, Ruoff C, Schroppel K, Naglik JR, Eckert SE, Mogensen EG, Haynes K, Tuite MF, Levin LR, Buck J, Muhlschlegel FA (2005) Fungal adenylyl cyclase integrates $\mathrm{CO}_{2}$ sensing with cAMP signaling and virulence. Curr Biol 15:2021-2026.

240. Knoepfler PS, Eisenman RN (1999) Sin meets NuRD and other tails of repression. Cell 99:447-450.

241. Kolle D, Sarg B, Lindner H, Loidl P (1998) Substrate and sequential site specificity of cytoplasmic histone acetyltransferases of maize and rat liver. FEBS Lett 421:109-114.

242. Korting HC, Hube B, Oberbauer S, Januschke E, Hamm G, Albrecht A, Borelli C, Schaller M (2003) Reduced expression of the hyphal-independent Candida albicans proteinase genes SAP1 and SAP3 in the efg 1 mutant is associated with attenuated virulence during infection of oral epithelium. J Med Microbiol 52:623632.

243. Kosodo Y, Noda Y, Adachi H, Yoda K (2003) Cooperation of Sly1/SM-family protein and sec18/NSF of Saccharomyces cerevisiae in disassembly of cis-SNARE membrane-protein complexes. Biosci Biotechnol Biochem 67:448-450.

244. Kouzarides T (2000) Acetylation: a regulatory modification to rival phosphorylation? Embo J 19:1176-1179.

245. Kouzarides $T$ (2007) Chromatin modifications and their function. Cell 128:693-705.

246. Krauke $\mathrm{Y}$, Sychrova $\mathrm{H}$ (2008) Functional comparison of plasma-membrane $\mathrm{Na}+/ \mathrm{H}+$ antiporters from two pathogenic Candida species. BMC Microbiol 8:80.

247. Krebs JE, Fry CJ, Samuels ML, Peterson CL (2000) Global role for chromatin remodeling enzymes in mitotic gene expression. Cell 102:587-598.

248. Krebs JE, Kuo MH, Allis CD, Peterson CL (1999) Cell cycle-regulated histone acetylation required for expression of the yeast HO gene. Genes Dev 13:14121421.

249. Kuo MH, Allis CD (1998) Roles of histone acetyltransferases and deacetylases in gene regulation. Bioessays 20:615-626.

250. Kuo MH, Brownell JE, Sobel RE, Ranalli TA, Cook RG, Edmondson DG, Roth SY, Allis CD (1996) Transcription-linked acetylation by Gcn5p of histones H3 and H4 at specific lysines. Nature 383:269-272.

251. Kuo MH, vom Baur E, Struhl K, Allis CD (2000) Gcn4 activator targets Gcn5 histone acetyltransferase to specific promoters independently of transcription. Mol Cell 6:1309-1320.

252. Kurdistani SK, Grunstein M (2003) Histone acetylation and deacetylation in yeast. Nat Rev Mol Cell Biol 4:276-284.

253. Kurdistani SK, Robyr D, Tavazoie S, Grunstein M (2002) Genome-wide binding map of the histone deacetylase Rpd3 in yeast. Nat Genet 31:248-254.

254. Kurtz MB, Kelly, M.R., and Kirsch, D.R. . (1990). Overview of Candida physiology, pathogenicity, and new anticandidal agents. In D. R. Kirsch, R. Kelly, and M.B. Kurtz (eds.), (Candida., T. g. o., Ed.), CRC Press, Florida.

255. Kusch H, Biswas K, Schwanfelder S, Engelmann S, Rogers PD, Hecker M, Morschhauser J (2004) A proteomic approach to understanding the development of multidrug-resistant Candida albicans strains. Mol Genet Genomics 271:554-565.

256. Kusch T, Florens L, Macdonald WH, Swanson SK, Glaser RL, Yates JR, 3rd, 
Abmayr SM, Washburn MP, Workman JL (2004) Acetylation by Tip60 is required for selective histone variant exchange at DNA lesions. Science 306:2084-2087.

257. Kvaal C, Lachke SA, Srikantha T, Daniels K, McCoy J, Soll DR (1999) Misexpression of the opaque-phase-specific gene PEP1 (SAP1) in the white phase of Candida albicans confers increased virulence in a mouse model of cutaneous infection. Infect Immun 67:6652-6662.

258. Lan CY, Newport G, Murillo LA, Jones T, Scherer S, Davis RW, Agabian N (2002) Metabolic specialization associated with phenotypic switching in Candida albicans. Proc Natl Acad Sci U S A 99:14907-14912.

259. Lan CY, Rodarte G, Murillo LA, Jones T, Davis RW, Dungan J, Newport G, Agabian N (2004) Regulatory networks affected by iron availability in Candida albicans. Mol Microbiol 53:1451-1469.

260. Land GA, McDonald WC, Stjernholm RL, Friedman L (1975) Factors affecting filamentation in Candida albicans: changes in respiratory activity of Candida albicans during filamentation. Infect Immun 12:119-127.

261. Lane S, Birse C, Zhou S, Matson R, Liu H (2001) DNA array studies demonstrate convergent regulation of virulence factors by Cph1, Cph2, and Efg1 in Candida albicans. J Biol Chem 276:48988-48996.

262. Lane S, Zhou S, Pan T, Dai Q, Liu H (2001) The basic helix-loop-helix transcription factor Cph2 regulates hyphal development in Candida albicans partly via TEC1. Mol Cell Biol 21:6418-6428.

263. Langner CA, Lodge JK, Travis SJ, Caldwell JE, Lu T, Li Q, Bryant ML, Devadas B, Gokel GW, Kobayashi GS, et al. (1992) 4-oxatetradecanoic acid is fungicidal for Cryptococcus neoformans and inhibits replication of human immunodeficiency virus I. J Biol Chem 267:17159-17169.

264. Lanternier F, Lortholary O (2008) Liposomal amphotericin B: what is its role in 2008? Clin Microbiol Infect 14 Suppl 4:71-83.

265. Leberer E, Harcus D, Dignard D, Johnson L, Ushinsky S, Thomas DY, Schroppel K (2001) Ras links cellular morphogenesis to virulence by regulation of the MAP kinase and CAMP signalling pathways in the pathogenic fungus Candida albicans. Mol Microbiol 42:673-687.

266. Lechner T, Carrozza MJ, Yu Y, Grant PA, Eberharter A, Vannier D, Brosch G, Stillman DJ, Shore D, Workman JL (2000) Sds3 (suppressor of defective silencing 3 ) is an integral component of the yeast Sin3[middle dot]Rpd3 histone deacetylase complex and is required for histone deacetylase activity. J Biol Chem 275:4096140966.

267. Lee CM, Nantel A, Jiang L, Whiteway M, Shen SH (2004) The serine/threonine protein phosphatase SIT4 modulates yeast-to-hypha morphogenesis and virulence in Candida albicans. Mol Microbiol 51:691-709.

268. Lee FJ, Stevens LA, Kao YL, Moss J, Vaughan M (1994) Characterization of a glucose-repressible ADP-ribosylation factor 3 (ARF3) from Saccharomyces cerevisiae. J Biol Chem 269:20931-20937.

269. Lee KK, Workman JL (2007) Histone acetyltransferase complexes: one size doesn't fit all. Nat Rev Mol Cell Biol 8:284-295.

270. Lee KL, Buckley HR, Campbell CC (1975) An amino acid liquid synthetic medium for the development of mycelial and yeast forms of Candida Albicans. Sabouraudia 13:148-153.

271. Lee KL, Rega ME, Campbell CC (1975) Amino acid synthetic media for fungal pathogens based on aminopeptidase specificities: Histoplasma capsulatum, Blastomyces dermititidis, Paracoccidioides brasiliensis and Cryptococcus neoformans. Sabouraudia 13:142-147.

272. Lee RE, Liu TT, Barker KS, Lee RE, Rogers PD (2005) Genome-wide expression profiling of the response to ciclopirox olamine in Candida albicans. J Antimicrob Chemother 55:655-662.

273. Leng P, Carter PE, Brown AJ (1998) The TATA-binding protein (TBP) from the human fungal pathogen Candida albicans can complement defects in human and 
yeast TBPs. J Bacteriol 180:1771-1776.

274. Lengeler KB, Tielker D, Ernst JF (2008) Protein-O-mannosyltransferases in virulence and development. Cell Mol Life Sci 65:528-544.

275. Lephart PR, Chibana H, Magee PT (2005) Effect of the major repeat sequence on chromosome loss in Candida albicans. Eukaryot Cell 4:733-741.

276. Li L, Rao JN, Guo X, Liu L, Santora R, Bass BL, Wang JY (2001) Polyamine depletion stabilizes p53 resulting in inhibition of normal intestinal epithelial cell proliferation. Am J Physiol Cell Physiol 281:C941-953.

277. Lin W, Srajer G, Evrard YA, Phan HM, Furuta Y, Dent SY (2007) Developmental potential of Gcn5(-/-) embryonic stem cells in vivo and in vitro. Dev Dyn 236:15471557.

278. Linsalata M, Russo F (2008) Nutritional factors and polyamine metabolism in colorectal cancer. Nutrition 24:382-389.

279. Liu H (2001) Transcriptional control of dimorphism in Candida albicans. Curr Opin Microbiol 4:728-735.

280. Liu H, Kohler J, Fink GR (1994) Suppression of hyphal formation in Candida albicans by mutation of a STE12 homolog. Science 266:1723-1726.

281. Liu TT, Lee RE, Barker KS, Lee RE, Wei L, Homayouni R, Rogers PD (2005) Genome-wide expression profiling of the response to azole, polyene, echinocandin, and pyrimidine antifungal agents in Candida albicans. Antimicrob Agents Chemother 49:2226-2236.

282. Liu TT, Znaidi S, Barker KS, Xu L, Homayouni R, Saidane S, Morschhauser J, Nantel A, Raymond M, Rogers PD (2007) Genome-wide expression and location analyses of the Candida albicans Tac1p regulon. Eukaryot Cell 6:2122-2138.

283. Loewith R, Smith JS, Meijer M, Williams TJ, Bachman N, Boeke JD, Young D (2001) Pho23 is associated with the Rpd3 histone deacetylase and is required for its normal function in regulation of gene expression and silencing in Saccharomyces cerevisiae. J Biol Chem 276:24068-24074.

284. Loidl P (2001) Introduction: assembly, remodeling and modification of chromatin. Cell Mol Life Sci 58:663-664.

285. Lopez MC, Garcia S, Ruiz-Herrera J, Dominguez A (1997) The ornithine decarboxylase gene from Candida albicans. Sequence analysis and expression during dimorphism. Curr Genet 32:108-114.

286. Lorenz MC, Bender JA, Fink GR (2004) Transcriptional response of Candida albicans upon internalization by macrophages. Eukaryot Cell 3:1076-1087.

287. Lorenz MC, Fink GR (2001) The glyoxylate cycle is required for fungal virulence. Nature 412:83-86.

288. Lorenz MC, Fink GR (2002) Life and death in a macrophage: role of the glyoxylate cycle in virulence. Eukaryot Cell 1:657-662.

289. Lucchini G, Hinnebusch AG, Chen C, Fink GR (1984) Positive regulatory interactions of the HIS4 gene of Saccharomyces cerevisiae. Mol Cell Biol 4:13261333.

290. Luger K, Mader AW, Richmond RK, Sargent DF, Richmond TJ (1997) Crystal structure of the nucleosome core particle at 2.8 A resolution. Nature 389:251-260.

291. Luger K, Richmond TJ (1998) The histone tails of the nucleosome. Curr Opin Genet Dev 8:140-146.

292. Lusser A, Kolle D, Loidl P (2001) Histone acetylation: lessons from the plant kingdom. Trends Plant Sci 6:59-65.

293. Lussier M, White,A.M., Sheraton,J., di Paolo,T., Treadwell,J., Southard,S.B., Horenstein,C.I., Chen-Weiner,J., and et al., (1997) Large scale identification of genes involved in cell surface biosynthesis and architecture in Saccharomyces cerevisiae. Genetics 147:435-450.

294. MacPherson S, Larochelle M, Turcotte B (2006) A fungal family of transcriptional regulators: the zinc cluster proteins. Microbiol Mol Biol Rev 70:583-604.

295. Maeda Y, Rachez C, Hawel L, 3rd, Byus CV, Freedman LP, Sladek FM (2002) Polyamines modulate the interaction between nuclear receptors and vitamin $D$ 
receptor-interacting protein 205. Mol Endocrinol 16:1502-1510.

296. Magee BB, Magee PT (2000) Induction of mating in Candida albicans by construction of MTLa and MTLalpha strains. Science 289:310-313.

297. Maglott D, Ostell J, Pruitt KD, Tatusova T (2007) Entrez Gene: gene-centered information at NCBI. Nucleic Acids Res 35:D26-31.

298. Maicas S, Moreno I, Nieto A, Gomez M, Sentandreu R, Valentin E (2005) In Silico Analysis for Transcription Factors With $\mathrm{Zn}(\mathrm{II})(2) \mathrm{C}(6)$ Binuclear Cluster DNA-Binding Domains in Candida albicans. Comp Funct Genomics 6:345-356.

299. Maidan MM, De Rop L, Serneels J, Exler S, Rupp S, Tournu H, Thevelein JM, Van Dijck P (2005) The G protein-coupled receptor Gpr1 and the Galpha protein Gpa2 act through the cAMP-protein kinase A pathway to induce morphogenesis in Candida albicans. Mol Biol Cell 16:1971-1986.

300. Mangold U, Leberer E (2005) Regulation of all members of the antizyme family by antizyme inhibitor. Biochem J 385:21-28.

301. Mao X, Cao F, Nie X, Liu H, Chen J (2006) The Swi/Snf chromatin remodeling complex is essential for hyphal development in Candida albicans. FEBS Lett 580:2615-2622.

302. Marchais V, Kempf M, Licznar P, Lefrancois C, Bouchara JP, Robert R, Cottin J (2005) DNA array analysis of Candida albicans gene expression in response to adherence to polystyrene. FEMS Microbiol Lett 245:25-32.

303. Markwardt DD, Garrett JM, Eberhardy S, Heideman W (1995) Activation of the Ras/cyclic AMP pathway in the yeast Saccharomyces cerevisiae does not prevent $\mathrm{G} 1$ arrest in response to nitrogen starvation. J Bacteriol 177:6761-6765.

304. Marmorstein R, Roth SY (2001) Histone acetyltransferases: function, structure, and catalysis. Curr Opin Genet Dev 11:155-161.

305. Marmorstein R, Trievel RC (2009) Histone modifying enzymes: Structures, mechanisms, and specificities. Biochim Biophys Acta 1789:58-68.

306. Martchenko M, Levitin A, Hogues H, Nantel A, Whiteway M (2007) Transcriptional rewiring of fungal galactose-metabolism circuitry. Curr Biol 17:1007-1013.

307. Martin N. (2006). Deacetilacion de histonas en C. albicans Analisis de su funcion en regulacion de la expresion geneica y morfogenesis. Doctoral Thesis, Salamanca.

308. Martinez-Iglesias O, Ruiz-Llorente L, Sanchez-Martinez R, Garcia L, Zambrano A, Aranda A (2008) Histone deacetylase inhibitors: mechanism of action and therapeutic use in cancer. Clin Transl Oncol 10:395-398.

309. Martinez Al, Castillo L, Garcera A, Elorza MV, Valentin E, Sentandreu R (2004) Role of Pir1 in the construction of the Candida albicans cell wall. Microbiology 150:3151-3161.

310. Martinez JP, Lopez-Ribot JL, Gil ML, Sentandreu R, Ruiz-Herrera J (1990) Inhibition of the dimorphic transition of Candida albicans by the ornithine decarboxylase inhibitor 1,4-diaminobutanone: alterations in the glycoprotein composition of the cell wall. J Gen Microbiol 136:1937-1943.

311. Martinez P, Ljungdahl PO (2005) Divergence of Stp1 and Stp2 transcription factors in Candida albicans places virulence factors required for proper nutrient acquisition under amino acid control. Mol Cell Biol 25:9435-9446.

312. Martins M, Henriques M, Azeredo J, Rocha SM, Coimbra MA, Oliveira R (2007) Morphogenesis control in Candida albicans and Candida dubliniensis through signaling molecules produced by planktonic and biofilm cells. Eukaryot Cell 6:24292436.

313. Marton LJ, Pegg AE (1995) Polyamines as targets for therapeutic intervention. Annu Rev Pharmacol Toxicol 35:55-91.

314. Mayes AE, Verdone L, Legrain P, Beggs JD (1999) Characterization of Sm-like proteins in yeast and their association with U6 snRNA. Embo J 18:4321-4331.

315. McCarroll SA, Murphy CT, Zou S, Pletcher SD, Chin CS, Jan YN, Kenyon C, Bargmann $\mathrm{Cl}$, Li H (2004) Comparing genomic expression patterns across species identifies shared transcriptional profile in aging. Nat Genet 36:197-204. 
316. McNemar MD, Gorman JA, Buckley HR (2001) Isolation of a gene encoding a putative polyamine transporter from Candida albicans, GPT1. Yeast 18:555-561.

317. Melo NR, Moran GP, Warrilow AG, Dudley E, Smith SN, Sullivan DJ, Lamb DC, Kelly DE, Coleman DC, Kelly SL (2008) CYP56 (Dit2p) in Candida albicans: characterization and investigation of its role in growth and antifungal drug susceptibility. Antimicrob Agents Chemother 52:3718-3724.

318. Meluh PB, Koshland D (1997) Budding yeast centromere composition and assembly as revealed by in vivo cross-linking. Genes Dev 11:3401-3412.

319. Meyskens FL, Jr., Gerner EW (1999) Development of difluoromethylornithine (DFMO) as a chemoprevention agent. Clin Cancer Res 5:945-951.

320. Michels CA, Read E, Nat K, Charron MJ (1992) The telomere-associated MAL3 locus of Saccharomyces is a tandem array of repeated genes. Yeast 8:655-665.

321. Mille C, Bobrowicz P, Trinel PA, Li H, Maes E, Guerardel Y, Fradin C, MartinezEsparza M, Davidson RC, Janbon G, Poulain D, Wildt S (2008) Identification of a new family of genes involved in beta-1,2-mannosylation of glycans in Pichia pastoris and Candida albicans. J Biol Chem 283:9724-9736.

322. Mio T, Adachi-Shimizu M, Tachibana Y, Tabuchi H, Inoue SB, Yabe T, YamadaOkabe T, Arisawa M, Watanabe T, Yamada-Okabe H (1997) Cloning of the Candida albicans homolog of Saccharomyces cerevisiae GSC1/FKS1 and its involvement in beta-1,3-glucan synthesis. J Bacteriol 179:4096-4105.

323. Mizzen CA, Allis CD (1998) Linking histone acetylation to transcriptional regulation. Cell Mol Life Sci 54:6-20.

324. Moreno I, Castillo L, Sentandreu R, Valentin E (2007) Global transcriptional profiling of Candida albicans cwt1 null mutant. Yeast 24:357-370.

325. Morris DR (1991) A new perspective on ornithine decarboxylase regulation: prevention of polyamine toxicity is the overriding theme. J Cell Biochem 46:102105.

326. Morschhauser J, Barker KS, Liu TT, Bla BWJ, Homayouni R, Rogers PD (2007) The transcription factor Mrr1p controls expression of the MDR1 efflux pump and mediates multidrug resistance in Candida albicans. PLoS Pathog 3:e164.

327. Muhlenhoff U, Stadler JA, Richhardt N, Seubert A, Eickhorst T, Schweyen RJ, Lill R, Wiesenberger G (2003) A specific role of the yeast mitochondrial carriers MRS3/4p in mitochondrial iron acquisition under iron-limiting conditions. J Biol Chem 278:40612-40620.

328. Muller EH, Richards EJ, Norbeck J, Byrne KL, Karlsson KA, Pretorius GH, Meacock PA, Blomberg A, Hohmann S (1999) Thiamine repression and pyruvate decarboxylase autoregulation independently control the expression of the Saccharomyces cerevisiae PDC5 gene. FEBS Lett 449:245-250.

329. Muniz M, Nuoffer C, Hauri HP, Riezman H (2000) The Emp24 complex recruits a specific cargo molecule into endoplasmic reticulum-derived vesicles. J Cell Biol 148:925-930.

330. Murad AM, d'Enfert C, Gaillardin C, Tournu H, Tekaia F, Talibi D, Marechal D, Marchais V, Cottin J, Brown AJ (2001) Transcript profiling in Candida albicans reveals new cellular functions for the transcriptional repressors CaTup1, CaMig1 and CaNrg1. Mol Microbiol 42:981-993.

331. Murad AM, Leng P, Straffon M, Wishart J, Macaskill S, MacCallum D, Schnell N, Talibi D, Marechal D, Tekaia F, d'Enfert C, Gaillardin C, Odds FC, Brown AJ (2001) NRG1 represses yeast-hypha morphogenesis and hypha-specific gene expression in Candida albicans. Embo J 20:4742-4752.

332. Murakami Y, Ichiba T, Matsufuji S, Hayashi S (1996) Cloning of antizyme inhibitor, a highly homologous protein to ornithine decarboxylase. J Biol Chem 271:33403342.

333. Murakami Y, Matsufuji S, Kameji T, Hayashi S, Igarashi K, Tamura T, Tanaka K, Ichihara A (1992) Ornithine decarboxylase is degraded by the $26 \mathrm{~S}$ proteasome without ubiquitination. Nature 360:597-599.

334. Murillo LA, Newport G, Lan CY, Habelitz S, Dungan J, Agabian NM (2005) 
Genome-wide transcription profiling of the early phase of biofilm formation by Candida albicans. Eukaryot Cell 4:1562-1573.

335. Murray K (1964) The Occurrence of Epsilon-N-Methyl Lysine in Histones. Biochemistry 3:10-15.

336. Nagarajan S, Ganem B, Pegg AE (1988) Studies of non-metabolizable polyamines that support growth of SV-3T3 cells depleted of natural polyamines by exposure to alpha-difluoromethylornithine. Biochem J 254:373-378.

337. Naglik JR, Challacombe SJ, Hube B (2003) Candida albicans secreted aspartyl proteinases in virulence and pathogenesis. Microbiol Mol Biol Rev 67:400-428, table of contents.

338. Naglik JR, Fostira F, Ruprai J, Staab JF, Challacombe SJ, Sundstrom P (2006) Candida albicans HWP1 gene expression and host antibody responses in colonization and disease. J Med Microbiol 55:1323-1327.

339. Naglik JR, Moyes D, Makwana J, Kanzaria P, Tsichlaki E, Weindl G, Tappuni AR, Rodgers CA, Woodman AJ, Challacombe SJ, Schaller M, Hube B (2008) Quantitative expression of the Candida albicans secreted aspartyl proteinase gene family in human oral and vaginal candidiasis. Microbiology 154:3266-3280.

340. Nakajima J, Papaah P, Yoshizawa M, Marotta F, Nakajima T, Mihara S, Minelli E (2007) Effect of a novel phyto-compound on mucosal candidiasis: further evidence from an ex vivo study. J Dig Dis 8:48-51.

341. Nakayama J, Rice JC, Strahl BD, Allis CD, Grewal SI (2001) Role of histone H3 lysine 9 methylation in epigenetic control of heterochromatin assembly. Science 292:110-113.

342. Nantel A, Dignard D, Bachewich C, Harcus D, Marcil A, Bouin AP, Sensen CW, Hogues $\mathrm{H}$, van het Hoog M, Gordon P, Rigby T, Benoit F, Tessier DC, Thomas DY, Whiteway M (2002) Transcription profiling of Candida albicans cells undergoing the yeast-to-hyphal transition. Mol Biol Cell 13:3452-3465.

343. Nather K, Munro CA (2008) Generating cell surface diversity in Candida albicans and other fungal pathogens. FEMS Microbiol Lett 285:137-145.

344. Navarro-Martinez MD, Cabezas-Herrera J, Rodriguez-Lopez JN (2006) Antifolates as antimycotics? Connection between the folic acid cycle and the ergosterol biosynthesis pathway in Candida albicans. Int J Antimicrob Agents 28:560-567.

345. Neuman-Silberberg FS, Bhattacharya S, Broach JR (1995) Nutrient availability and the RAS/cyclic AMP pathway both induce expression of ribosomal protein genes in Saccharomyces cerevisiae but by different mechanisms. Mol Cell Biol 15:31873196.

346. Neuwald AF, Landsman D (1997) GCN5-related histone N-acetyltransferases belong to a diverse superfamily that includes the yeast SPT10 protein. Trends Biochem Sci 22:154-155.

347. Nieto A, Sanz P, Sentandreu R, del Castillo Agudo L (1993) Cloning and characterization of the SEC18 gene from Candida albicans. Yeast 9:875-887.

348. Nilsson JA, Keller UB, Baudino TA, Yang C, Norton S, Old JA, Nilsson LM, Neale G, Kramer DL, Porter CW, Cleveland JL (2005) Targeting ornithine decarboxylase in Myc-induced lymphomagenesis prevents tumor formation. Cancer Cell 7:433444.

349. Nilsson JA, Maclean KH, Keller UB, Pendeville H, Baudino TA, Cleveland JL (2004) Mnt loss triggers Myc transcription targets, proliferation, apoptosis, and transformation. Mol Cell Biol 24:1560-1569.

350. Nino-Vega GA, Sorais F, Calcagno AM, Ruiz-Herrera J, Martinez-Espinoza AD, San-Blas G (2004) Cloning and expression analysis of the ornithine decarboxylase gene (PbrODC) of the pathogenic fungus Paracoccidioides brasiliensis. Yeast 21:211-218.

351. Nishimura K, Murozumi K, Shirahata A, Park MH, Kashiwagi K, Igarashi K (2005) Independent roles of elF5A and polyamines in cell proliferation. Biochem $\mathrm{J}$ 385:779-785.

352. Nishizuka Y, Ueda K, Honjo T, Hayaishi O (1968) Enzymic adenosine diphosphate 
ribosylation of histone and poly adenosine diphosphate ribose synthesis in rat liver nuclei. J Biol Chem 243:3765-3767.

353. Nobile CJ, Bruno VM, Richard ML, Davis DA, Mitchell AP (2003) Genetic control of chlamydospore formation in Candida albicans. Microbiology 149:3629-3637.

354. Nobile CJ, Mitchell AP (2005) Regulation of cell-surface genes and biofilm formation by the $C$. albicans transcription factor Bcr1p. Curr Biol 15:1150-1155.

355. Nobile CJ, Schneider HA, Nett JE, Sheppard DC, Filler SG, Andes DR, Mitchell AP (2008) Complementary adhesin function in C. albicans biofilm formation. Curr Biol 18:1017-1024.

356. Norice CT, Smith FJ, Jr., Solis N, Filler SG, Mitchell AP (2007) Requirement for Candida albicans Sun41 in biofilm formation and virulence. Eukaryot Cell 6:20462055.

357. Nosaka K (2006) Recent progress in understanding thiamin biosynthesis and its genetic regulation in Saccharomyces cerevisiae. Appl Microbiol Biotechnol 72:3040.

358. Odds FC. (1988). Candida and candidosis (ed, n., Ed.), Baillière Tindall, London, United Kingdom.

359. Oliver BG, Song JL, Choiniere JH, White TC (2007) cis-Acting elements within the Candida albicans ERG11 promoter mediate the azole response through transcription factor Upc2p. Eukaryot Cell 6:2231-2239.

360. Onyewu C, Wormley FL, Jr., Perfect JR, Heitman J (2004) The calcineurin target, Crz1, functions in azole tolerance but is not required for virulence of Candida albicans. Infect Immun 72:7330-7333.

361. Packham G, Cleveland JL (1994) Ornithine decarboxylase is a mediator of c-Mycinduced apoptosis. Mol Cell Biol 14:5741-5747.

362. Packham G, Cleveland JL (1995) The role of ornithine decarboxylase in c-Mycinduced apoptosis. Curr Top Microbiol Immunol 194:283-290.

363. Park JH, Aravind L, Wolff EC, Kaevel J, Kim YS, Park MH (2006) Molecular cloning, expression, and structural prediction of deoxyhypusine hydroxylase: a HEAT-repeat-containing metalloenzyme. Proc Natl Acad Sci U S A 103:51-56.

364. Park JY, Kim DH, Shin WS, Seo HS, Kim K, Lee KH, Koh CM, Bong JP (2003) Search for genes potentially related to germ tube formation in Candida albicans by differential-display reverse transcription polymerase chain reaction. Yonsei Med J 44:110-118.

365. Pegg AE (2006) Regulation of ornithine decarboxylase. J Biol Chem 281:1452914532.

366. Pelham HR, Hardwick KG, Lewis MJ (1988) Sorting of soluble ER proteins in yeast. Embo J 7:1757-1762.

367. Peter C, Barth C, Petri E, Martin E, Walther A (2008) Spermine and endothelial damage during endotoxemia. Inflammation 31:208-213.

368. Pirinen E, Kuulasmaa T, Pietila M, Heikkinen S, Tusa M, Itkonen P, Boman S, Skommer J, Virkamaki A, Hohtola E, Kettunen M, Fatrai S, Kansanen E, Koota S, Niiranen K, Parkkinen J, Levonen AL, Yla-Herttuala S, Hiltunen JK, Alhonen L, Smith U, Janne J, Laakso M (2007) Enhanced polyamine catabolism alters homeostatic control of white adipose tissue mass, energy expenditure, and glucose metabolism. Mol Cell Biol 27:4953-4967.

369. Pollard KJ, Peterson CL (1997) Role for ADA/GCN5 products in antagonizing chromatin-mediated transcriptional repression. Mol Cell Biol 17:6212-6222.

370. Porta A, Ramon AM, Fonzi WA (1999) PRR1, a homolog of Aspergillus nidulans palF, controls $\mathrm{pH}$-dependent gene expression and filamentation in Candida albicans. J Bacteriol 181:7516-7523.

371. Poulin R, Pelletier G, Pegg AE (1995) Induction of apoptosis by excessive polyamine accumulation in ornithine decarboxylase-overproducing L1210 cells. Biochem J 311 ( Pt 3):723-727.

372. Prasad R, De Wergifosse P, Goffeau A, Balzi E (1995) Molecular cloning and characterization of a novel gene of Candida albicans, CDR1, conferring multiple 
resistance to drugs and antifungals. Curr Genet 27:320-329.

373. Prigneau O, Porta A, Poudrier JA, Colonna-Romano S, Noel T, Maresca B (2003) Genes involved in beta-oxidation, energy metabolism and glyoxylate cycle are induced by Candida albicans during macrophage infection. Yeast 20:723-730.

374. Qi D, Larsson J, Mannervik M (2004) Drosophila Ada2b is required for viability and normal histone $\mathrm{H} 3$ acetylation. Mol Cell Biol 24:8080-8089.

375. Ram AF, Wolters A, Ten Hoopen R, Klis FM (1994) A new approach for isolating cell wall mutants in Saccharomyces cerevisiae by screening for hypersensitivity to calcofluor white. Yeast 10:1019-1030.

376. Ramanan N, Wang Y (2000) A high-affinity iron permease essential for Candida albicans virulence. Science 288:1062-1064.

377. Ramsdale M, Selway L, Stead D, Walker J, Yin Z, Nicholls SM, Crowe J, Sheils EM, Brown AJ (2008) MNL1 regulates weak acid-induced stress responses of the fungal pathogen Candida albicans. Mol Biol Cell 19:4393-4403.

378. Rashki Z. (2009). Global transcriptional response of C. albicans to histone acetyltransferases deletion. Doctoral Thesis Salamanca.

379. Rauceo JM, Blankenship JR, Fanning S, Hamaker JJ, Deneault JS, Smith FJ, Nantel A, Mitchell AP (2008) Regulation of the Candida albicans cell wall damage response by transcription factor Sko1 and PAS kinase Psk1. Mol Biol Cell 19:27412751.

380. Rea S, Eisenhaber F, O'Carroll D, Strahl BD, Sun ZW, Schmid M, Opravil S, Mechtler K, Ponting CP, Allis CD, Jenuwein T (2000) Regulation of chromatin structure by site-specific histone H3 methyltransferases. Nature 406:593-599.

381. Reid JL, lyer VR, Brown PO, Struhl K (2000) Coordinate regulation of yeast ribosomal protein genes is associated with targeted recruitment of Esa1 histone acetylase. Mol Cell 6:1297-1307.

382. Reifnyder C, Lowell J, Clarke A, Pillus L (1997) Yeast SAS silencing genes and human genes associated with AML and HIV-1 Tat interactions are homologous with acetyltransferases. Nat Genet 16:109.

383. Reuss O, Morschhauser $\mathrm{J}$ (2006) A family of oligopeptide transporters is required for growth of Candida albicans on proteins. Mol Microbiol 60:795-812.

384. Rifkin SA, Kim J, White KP (2003) Evolution of gene expression in the Drosophila melanogaster subgroup. Nat Genet 33:138-144.

385. Robert F, Pokholok DK, Hannett NM, Rinaldi NJ, Chandy M, Rolfe A, Workman JL, Gifford DK, Young RA (2004) Global position and recruitment of HATs and HDACs in the yeast genome. Mol Cell 16:199-209.

386. Robyr D, Suka Y, Xenarios I, Kurdistani SK, Wang A, Suka N, Grunstein M (2002) Microarray deacetylation maps determine genome-wide functions for yeast histone deacetylases. Cell 109:437-446.

387. Robzyk K, Recht J, Osley MA (2000) Rad6-dependent ubiquitination of histone H2B in yeast. Science 287:501-504.

388. Rocha CR, Schroppel K, Harcus D, Marcil A, Dignard D, Taylor BN, Thomas DY, Whiteway M, Leberer E (2001) Signaling through adenylyl cyclase is essential for hyphal growth and virulence in the pathogenic fungus Candida albicans. Mol Biol Cell 12:3631-3643.

389. Rodriguez-Kessler M, Ruiz OA, Maiale S, Ruiz-Herrera J, Jimenez-Bremont JF (2008) Polyamine metabolism in maize tumors induced by Ustilago maydis. Plant Physiol Biochem 46:805-814.

390. Rogers PD, Barker KS (2003) Genome-wide expression profile analysis reveals coordinately regulated genes associated with stepwise acquisition of azole resistance in Candida albicans clinical isolates. Antimicrob Agents Chemother 47:1220-1227.

391. Rose MDW, F; Hieter, P, Ed. (1990). Methods in Yeast Genetics : A Laboratory Manual: Plainview, NY: Cold Spring Harbor Lab. Press.

392. Roth SY, Denu JM, Allis CD (2001) Histone acetyltransferases. Annu Rev Biochem 70:81-120. 
393. Rubin-Bejerano I, Fraser I, Grisafi P, Fink GR (2003) Phagocytosis by neutrophils induces an amino acid deprivation response in Saccharomyces cerevisiae and Candida albicans. Proc Natl Acad Sci U S A 100:11007-11012.

394. Ruiz-Carrillo A, Wangh LJ, Allfrey VG (1975) Processing of newly synthesized histone molecules. Science 190:117-128.

395. Ruiz-Herrera J (1994) Polyamines, DNA methylation, and fungal differentiation. Crit Rev Microbiol 20:143-150.

396. Rundlett SE, Carmen AA, Kobayashi R, Bavykin S, Turner BM, Grunstein M (1996) $H D A 1$ and $R P D 3$ are members of distinct yeast histone deacetylase complexes that regulate silencing and transcription. Proc Natl Acad Sci U S A 93:14503-14508.

397. Rustici G, Mata J, Kivinen K, Lio P, Penkett CJ, Burns G, Hayles J, Brazma A, Nurse P, Bahler J (2004) Periodic gene expression program of the fission yeast cell cycle. Nat Genet 36:809-817.

398. Salama NR, Chuang JS, Schekman RW (1997) Sec31 encodes an essential component of the COPII coat required for transport vesicle budding from the endoplasmic reticulum. Mol Biol Cell 8:205-217.

399. Sambrook J, David, W., Russsell. (2001). Molecular cloning: a laboratory manual, (ed., n., Ed.), Cold Spring Harbor Laboratory, Cold Spring Harbor, N.Y.

400. Sambrook J, E. F. Fritsch, and T. Maniatis. (1989). Molecular cloning: a laboratory manual, (ed, n., Ed.), Cold Spring Harbor Laboratory, Cold Spring Harbor, N.Y.

401. Samonis G, Kofteridis DP, Saloustros E, Giannopoulou KP, Ntziora F, Christidou A, Maraki S, Falagas ME (2008) Candida albicans versus non-albicans bloodstream infection in patients in a tertiary hospital: an analysis of microbiological data. Scand $J$ Infect Dis 40:414-419.

402. San-Blas G, Sorais F, San-Blas F, Ruiz-Herrera J (1996) Ornithine decarboxylase in Paracoccidioides brasiliensis. Arch Microbiol 165:311-316.

403. Sanchez-Martinez C, Perez-Martin J (2002) Gpa2, a G-protein alpha subunit required for hyphal development in Candida albicans. Eukaryot Cell 1:865-874.

404. Santos-Rosa H, Caldas C (2005) Chromatin modifier enzymes, the histone code and cancer. Eur J Cancer 41:2381-2402.

405. Sassone-Corsi P, Mizzen CA, Cheung P, Crosio C, Monaco L, Jacquot S, Hanauer A, Allis CD (1999) Requirement of Rsk-2 for epidermal growth factor-activated phosphorylation of histone H3. Science 285:886-891.

406. Schaller M, Bein M, Korting HC, Baur S, Hamm G, Monod M, Beinhauer S, Hube B (2003) The secreted aspartyl proteinases Sap1 and Sap2 cause tissue damage in an in vitro model of vaginal candidiasis based on reconstituted human vaginal epithelium. Infect Immun 71:3227-3234.

407. Schaller M, Schackert C, Korting HC, Januschke E, Hube B (2000) Invasion of Candida albicans correlates with expression of secreted aspartic proteinases during experimental infection of human epidermis. J Invest Dermatol 114:712-717.

408. Schaller M, Weindl G (2009) Models of oral and vaginal candidiasis based on in vitro reconstituted human epithelia for the study of host-pathogen interactions. Methods Mol Biol 470:327-345.

409. Schinabeck MK, Long LA, Hossain MA, Chandra J, Mukherjee PK, Mohamed S, Ghannoum MA (2004) Rabbit model of Candida albicans biofilm infection: liposomal amphotericin B antifungal lock therapy. Antimicrob Agents Chemother 48:1727-1732.

410. Schofield DA, Westwater C, Warner T, Balish E (2005) Differential Candida albicans lipase gene expression during alimentary tract colonization and infection. FEMS Microbiol Lett 244:359-365.

411. Schreiber-Agus N, Chin L, Chen K, Torres R, Rao G, Guida P, Skoultchi Al, DePinho RA (1995) An amino-terminal domain of Mxi1 mediates anti-Myc oncogenic activity and interacts with a homolog of the yeast transcriptional repressor SIN3. Cell 80:777-786.

412. Semenza JC, Hardwick KG, Dean N, Pelham HR (1990) ERD2, a yeast gene required for the receptor-mediated retrieval of luminal ER proteins from the 
secretory pathway. Cell 61:1349-1357.

413. Seneviratne CJ, Jin LJ, Samaranayake YH, Samaranayake LP (2008) Cell density and cell aging as factors modulating antifungal resistance of Candida albicans biofilms. Antimicrob Agents Chemother 52:3259-3266.

414. Sengupta N, Seto E (2004) Regulation of histone deacetylase activities. J Cell Biochem 93:57-67.

415. Sennhenn-Kirchner S, Schwarz P, Schliephake H, Konietschke F, Brunner E, Borgvon Zepelin M (2008) Decontamination efficacy of erbium:yttrium-aluminium-garnet and diode laser light on oral Candida albicans isolates of a 5-day in vitro biofilm model. Lasers Med Sci.

416. Sentandreu M, Nieto A, Iborra A, Elorza MV, Ponton J, Fonzi WA, Sentandreu R (1997) Cloning and characterization of CSP37, a novel gene encoding a putative membrane protein of Candida albicans. J Bacteriol 179:4654-4663.

417. Serefko A, Los R, Biernasiuk A, Malm A (2008) Comparison of microdilution method and E-test procedure in susceptibility testing of caspofungin against Candida non-albicans species. New Microbiol 31:257-262.

418. Shantz LM, Pegg AE (1999) Translational regulation of ornithine decarboxylase and other enzymes of the polyamine pathway. Int J Biochem Cell Biol 31:107-122.

419. Sharkey LL, McNemar MD, Saporito-Irwin SM, Sypherd PS, Fonzi WA (1999) HWP1 functions in the morphological development of Candida albicans downstream of EFG1, TUP1, and RBF1. J Bacteriol 181:5273-5279.

420. Shaywitz DA, Espenshade PJ, Gimeno RE, Kaiser CA (1997) COPII subunit interactions in the assembly of the vesicle coat. J Biol Chem 272:25413-25416.

421. Shea M, Jakle U, Liu Q, Berry C, Joiner KA, Soldati-Favre D (2007) A family of aspartic proteases and a novel, dynamic and cell-cycle-dependent protease localization in the secretory pathway of Toxoplasma gondii. Traffic 8:1018-1034.

422. Sheppard DC, Yeaman MR, Welch WH, Phan QT, Fu Y, Ibrahim AS, Filler SG, Zhang M, Waring AJ, Edwards JE, Jr. (2004) Functional and structural diversity in the Als protein family of Candida albicans. J Biol Chem 279:30480-30489.

423. Shimogori T, Kashiwagi K, Igarashi K (1996) Spermidine regulation of protein synthesis at the level of initiation complex formation of Met-tRNAi, mRNA and ribosomes. Biochem Biophys Res Commun 223:544-548.

424. Sigle HC, Thewes S, Niewerth M, Korting HC, Schafer-Korting M, Hube B (2005) Oxygen accessibility and iron levels are critical factors for the antifungal action of ciclopirox against Candida albicans. J Antimicrob Chemother 55:663-673.

425. Silveira LA, Wong DH, Masiarz FR, Schekman R (1990) Yeast clathrin has a distinctive light chain that is important for cell growth. J Cell Biol 111:1437-1449.

426. Simonetti G, Passariello C, Rotili D, Mai A, Garaci E, Palamara AT (2007) Histone deacetylase inhibitors may reduce pathogenicity and virulence in Candida albicans. FEMS Yeast Res 7:1371-1380.

427. Singh V, Sinha I, Sadhale PP (2005) Global analysis of altered gene expression during morphogenesis of Candida albicans in vitro. Biochem Biophys Res Commun 334:1149-1158.

428. Sinha I, Wang YM, Philp R, Li CR, Yap WH, Wang Y (2007) Cyclin-dependent kinases control septin phosphorylation in Candida albicans hyphal development. Dev Cell 13:421-432.

429. Slesarev AI, Belova GI, Kozyavkin SA, Lake JA (1998) Evidence for an early prokaryotic origin of histones $\mathrm{H} 2 \mathrm{~A}$ and $\mathrm{H} 4$ prior to the emergence of eukaryotes. Nucleic Acids Res 26:427-430.

430. Slutsky B, Buffo J, Soll DR (1985) High-frequency switching of colony morphology in Candida albicans. Science 230:666-669.

431. Slutsky B, Staebell M, Anderson J, Risen L, Pfaller M, Soll DR (1987) "Whiteopaque transition": a second high-frequency switching system in Candida albicans. J Bacteriol 169:189-197.

432. Smriti, Krishnamurthy S, Dixit BL, Gupta CM, Milewski S, Prasad R (2002) ABC transporters Cdr1p, Cdr2p and Cdr3p of a human pathogen Candida albicans are 
general phospholipid translocators. Yeast 19:303-318.

433. Snapir Z, Keren-Paz A, Bercovich Z, Kahana C (2008) ODCp, a brain- and testisspecific ornithine decarboxylase paralogue, functions as an antizyme inhibitor, although less efficiently than Azl1. Biochem J 410:613-619.

434. Sohn K, Urban C, Brunner H, Rupp S (2003) EFG1 is a major regulator of cell wall dynamics in Candida albicans as revealed by DNA microarrays. Mol Microbiol 47:89-102.

435. Soll DR (1997) Gene regulation during high-frequency switching in Candida albicans. Microbiology 143 ( Pt 2):279-288.

436. Soll DR (1992) High-frequency switching in Candida albicans. Clin Microbiol Rev 5:183-203.

437. Soll DR. (2001). Phenotypic switching. In R. Calderone (ed.), Candida and Candidiasis, American Society for Microbiology, Washington, D. C.

438. Soll DR. (1992b). Switching and its possible role in Candida pathogenesis. In J. E. Bennet, R. J. Hay, and P. K. Peterson (ed.), New strategies in fungal disease., Churchill Livingstone, . Edinburgh, United Kingdom.

439. Song Y, Cheon SA, Lee KE, Lee SY, Lee BK, Oh DB, Kang HA, Kim JY (2008) Role of the RAM network in cell polarity and hyphal morphogenesis in Candida albicans. Mol Biol Cell 19:5456-5477.

440. Sonneborn A, Tebarth B, Ernst JF (1999) Control of white-opaque phenotypic switching in Candida albicans by the Efg1p morphogenetic regulator. Infect Immun 67:4655-4660.

441. Sosinska GJ, de Groot PW, Teixeira de Mattos MJ, Dekker HL, de Koster CG, Hellingwerf KJ, Klis FM (2008) Hypoxic conditions and iron restriction affect the cell-wall proteome of Candida albicans grown under vagina-simulative conditions. Microbiology 154:510-520.

442. Srivastava Alka SHC, a, Tahira Fatima Tatsiana Datsenka,, Avtar K. Handa, Autar K. Mattoo, (2007) Polyamines as anabolic growth regulators revealed by transcriptome analysis and metabolite profiles of tomato fruits engineered to accumulate spermidine and spermine. Plant Biotechnology 24:57-70.

443. Staab JF, Bradway SD, Fidel PL, Sundstrom P (1999) Adhesive and mammalian transglutaminase substrate properties of Candida albicans Hwp1. Science 283:1535-1538.

444. Staab JF, Ferrer CA, Sundstrom P (1996) Developmental expression of a tandemly repeated, proline-and glutamine-rich amino acid motif on hyphal surfaces on Candida albicans. J Biol Chem 271:6298-6305.

445. Staib P, Kretschmar M, Nichterlein T, Kohler G, Michel S, Hof H, Hacker J, Morschhauser J (1999) Host-induced, stage-specific virulence gene activation in Candida albicans during infection. Mol Microbiol 32:533-546.

446. Staib P, Morschhauser J (2007) Chlamydospore formation in Candida albicans and Candida dubliniensis -an enigmatic developmental programme. Mycoses 50:1-12.

447. Stehr F, Felk A, Kretschmar M, Schaller M, Schafer W, Hube B (2000) [Extracellular hydrolytic enzymes and their relevance during Candida albicans infections.]. Mycoses 43 Suppl 2:17-21.

448. Sterner DE, Berger SL (2000) Acetylation of histones and transcription-related factors. Microbiol Mol Biol Rev 64:435-459.

449. Struhl K (1998) Histone acetylation and transcriptional regulatory mechanisms. Genes Dev 12:599-606.

450. Stuart JM, Segal E, Koller D, Kim SK (2003) A gene-coexpression network for global discovery of conserved genetic modules. Science 302:249-255.

451. Sudbery P, Gow N, Berman J (2004) The distinct morphogenic states of Candida albicans. Trends Microbiol 12:317-324.

452. Suka N, Luo K, Grunstein M (2002) Sir2p and Sas2p opposingly regulate acetylation of yeast histone $\mathrm{H} 4$ lysine 16 and spreading of heterochromatin. Nat Genet 32:378-383.

453. Suka N, Suka Y, Carmen AA, Wu J, Grunstein M (2001) Highly specific antibodies 
determine histone acetylation site usage in yeast heterochromatin and euchromatin. Mol Cell 8:473-479.

454. Sundstrom P (1999) Adhesins in Candida albicans. Curr Opin Microbiol 2:353-357.

455. Sundstrom P (2002) Adhesion in Candida spp. Cell Microbiol 4:461-469.

456. Sundstrom P. (2006). Candida albicans hypha formation and virulence. In Molecular Principles of Fungal Pathogenesis, DC: American Society for Microbiology, Washington.

457. Sutherland DJ, Tsang BK, Merali Z, Singhal RL (1974) Testicular and prostatic cyclic amp metabolism following chronic cadmium treatment and subsequent withdrawal. Environ Physiol Biochem 4:205-213.

458. Svensson KJ, Welch JE, Kucharzewska P, Bengtson P, Bjurberg M, Pahlman S, Ten Dam GB, Persson L, Belting M (2008) Hypoxia-mediated induction of the polyamine system provides opportunities for tumor growth inhibition by combined targeting of vascular endothelial growth factor and ornithine decarboxylase. Cancer Res 68:9291-9301.

459. Swigart P, Insall R, Wilkins A, Cockcroft S (2000) Purification and cloning of phosphatidylinositol transfer proteins from Dictyostelium discoideum: homologues of both mammalian PITPs and Saccharomyces cerevisiae sec14p are found in the same cell. Biochem J 347 Pt 3:837-843.

460. Swoboda RK, Bertram G, Delbruck S, Ernst JF, Gow NA, Gooday GW, Brown AJ (1994) Fluctuations in glycolytic mRNA levels during morphogenesis in Candida albicans reflect underlying changes in growth and are not a response to cellular dimorphism. Mol Microbiol 13:663-672.

461. Syntichaki P, Thireos G (1998) The Gcn5.Ada complex potentiates the histone acetyltransferase activity of Gcn5. J Biol Chem 273:24414-24419.

462. Taunton J, Hassig CA, Schreiber SL (1996) A mammalian histone deacetylase related to the yeast transcriptional regulator Rpd3p. Science 272:408-411.

463. Tebarth B, Doedt T, Krishnamurthy S, Weide M, Monterola F, Dominguez A, Ernst JF (2003) Adaptation of the Efg1p morphogenetic pathway in Candida albicans by negative autoregulation and PKA-dependent repression of the EFG1 gene. J Mol Biol 329:949-962.

464. Terasaki AG, Morikawa K, Suzuki H, Oshima K, Ohashi K (2002) Characterization of Arp2/3 complex in chicken tissues. Cell Struct Funct 27:383-391.

465. Thatcher TH, Gorovsky MA (1994) Phylogenetic analysis of the core histones H2A, H2B, H3, and H4. Nucleic Acids Res 22:174-179.

466. Thewes S, Kretschmar M, Park H, Schaller M, Filler SG, Hube B (2007) In vivo and ex vivo comparative transcriptional profiling of invasive and non-invasive Candida albicans isolates identifies genes associated with tissue invasion. Mol Microbiol 63:1606-1628.

467. Thomas D, Bron P, Weimann T, Dautant A, Giraud MF, Paumard P, Salin B, Cavalier A, Velours J, Brethes D (2008) Supramolecular organization of the yeast F1Fo-ATP synthase. Biol Cell 100:591-601.

468. Thomas T, Thomas TJ (2001) Polyamines in cell growth and cell death: molecular mechanisms and therapeutic applications. Cell Mol Life Sci 58:244-258.

469. Thomson S, Clayton AL, Hazzalin CA, Rose S, Barratt MJ, Mahadevan LC (1999) The nucleosomal response associated with immediate-early gene induction is mediated via alternative MAP kinase cascades: MSK1 as a potential histone H3/HMG-14 kinase. Embo J 18:4779-4793.

470. Timpel C, Zink S, Strahl-Bolsinger S, Schroppel K, Ernst J (2000) Morphogenesis, adhesive properties, and antifungal resistance depend on the Pmt6 protein mannosyltransferase in the fungal pathogen candida albicans. J Bacteriol 182:3063-3071.

471. Tirosh I, Barkai N (2008) Two strategies for gene regulation by promoter nucleosomes. Genome Res 18:1084-1091.

472. Tripathi G, Wiltshire C, Macaskill S, Tournu H, Budge S, Brown AJ (2002) Gcn4 coordinates morphogenetic and metabolic responses to amino acid starvation in 
Candida albicans. Embo J 21:5448-5456.

473. Tsukada $\mathrm{Y}$, Fang J, Erdjument-Bromage $\mathrm{H}$, Warren ME, Borchers $\mathrm{CH}$, Tempst $\mathrm{P}$, Zhang $Y$ (2006) Histone demethylation by a family of JmjC domain-containing proteins. Nature 439:811-816.

474. Turner BM (2000) Histone acetylation and an epigenetic code. Bioessays 22:836845.

475. Tyagi AK, Tabor CW, Tabor H (1981) Ornithine decarboxylase from Saccharomyces cerevisiae. Purification, properties, and regulation of activity. J Biol Chem 256:12156-12163.

476. Ueno Y, Fukumatsu M, Ogasawara A, Watanabe T, Mikami T, Matsumoto T (2004) Hyphae formation of Candida albicans is regulated by polyamines. Biol Pharm Bull 27:890-892.

477. Uhl MA, Biery M, Craig N, Johnson AD (2003) Haploinsufficiency-based large-scale forward genetic analysis of filamentous growth in the diploid human fungal pathogen C.albicans. Embo J 22:2668-2678.

478. Uhrig JF, Mutondo M, Zimmermann I, Deeks MJ, Machesky LM, Thomas P, Uhrig S, Rambke C, Hussey PJ, Hulskamp M (2007) The role of Arabidopsis SCAR genes in ARP2-ARP3-dependent cell morphogenesis. Development 134:967-977.

479. Utley RT, Ikeda K, Grant PA, Cote J, Steger DJ, Eberharter A, John S, Workman JL (1998) Transcriptional activators direct histone acetyltransferase complexes to nucleosomes. Nature 394:498-502.

480. Utley RTC, J. (2002) The MYST family of histone acetyltransferases. Curr. Top. Microbiol. Immunol. 274, 203-236 (2002). Curr. Top. Microbiol. Immunol.

274:,203-236

481. Van den Bossche H (2002) Equinocandins - an update. Expert Opin Ther Pat 12.

482. van het Hoog M, Rast TJ, Martchenko M, Grindle S, Dignard D, Hogues H, Cuomo C, Berriman M, Scherer S, Magee BB, Whiteway M, Chibana H, Nantel A, Magee PT (2007) Assembly of the Candida albicans genome into sixteen supercontigs aligned on the eight chromosomes. Genome Biol 8:R52.

483. Vannier-Santos MA, Menezes D, Oliveira MF, de Mello FG (2008) The putrescine analogue 1,4-diamino-2-butanone affects polyamine synthesis, transport, ultrastructure and intracellular survival in Leishmania amazonensis. Microbiology 154:3104-3111.

484. Varma A, Singh BB, Karnani N, Lichtenberg-Frate H, Hofer M, Magee BB, Prasad $\mathrm{R}$ (2000) Molecular cloning and functional characterisation of a glucose transporter, CaHGT1, of Candida albicans. FEMS Microbiol Lett 182:15-21.

485. Vazquez JAS, J.D. Candidiasis. In: Dismukes WE, Pappas PG, Sobel JD. (2003). Candidiasis. In: Dismukes WE, Pappas PG, Sobel JD., Clinical Mycology New York, NY: Oxford University Press.

486. Veen M, Stahl U, Lang C (2003) Combined overexpression of genes of the ergosterol biosynthetic pathway leads to accumulation of sterols in Saccharomyces cerevisiae. FEMS Yeast Res 4:87-95.

487. Velours G, Boucheron C, Manon S, Camougrand N (2002) Dual cell wall/mitochondria localization of the 'SUN' family proteins. FEMS Microbiol Lett 207:165-172.

488. Vernarecci S, Ornaghi P, Bagu A, Cundari E, Ballario P, Filetici P (2008) Gcn5p plays an important role in centromere kinetochore function in budding yeast. Mol Cell Biol 28:988-996.

489. Vogelauer M, Wu J, Suka N, Grunstein M (2000) Global histone acetylation and deacetylation in yeast. Nature 408:495-498.

490. Wahlfors J, Alhonen L, Kauppinen L, Hyvonen T, Janne J, Eloranta TO (1990) Human spermidine synthase: cloning and primary structure. DNA Cell Biol 9:103110.

491. Wallace HM (2000) The physiological role of the polyamines. Eur J Clin Invest 30:13.

492. Wallace HM, Fraser AV, Hughes A (2003) A perspective of polyamine metabolism. 
Biochem J 376:1-14.

493. Wang X, Ikeguchi $Y$, McCloskey DE, Nelson P, Pegg AE (2004) Spermine synthesis is required for normal viability, growth, and fertility in the mouse. J Biol Chem 279:51370-51375.

494. Wang Y, Cao YY, Cao YB, Wang DJ, Jia XM, Fu XP, Zhang JD, Xu Z, Ying K, Chen WS, Jiang YY (2007) Cap1p plays regulation roles in redox, energy metabolism and substance transport: an investigation on Candida albicans under normal culture condition. Front Biosci 12:145-153.

495. Wang Y, Cao YY, Jia XM, Cao YB, Gao PH, Fu XP, Ying K, Chen WS, Jiang YY (2006) Cap1p is involved in multiple pathways of oxidative stress response in Candida albicans. Free Radic Biol Med 40:1201-1209.

496. Wang Y, Wysocka J, Sayegh J, Lee YH, Perlin JR, Leonelli L, Sonbuchner LS, McDonald CH, Cook RG, Dou Y, Roeder RG, Clarke S, Stallcup MR, Allis CD, Coonrod SA (2004) Human PAD4 regulates histone arginine methylation levels via demethylimination. Science 306:279-283.

497. Warenda AJ, Konopka JB (2002) Septin function in Candida albicans morphogenesis. Mol Biol Cell 13:2732-2746.

498. Weissman Z, Kornitzer D (2004) A family of Candida cell surface haem-binding proteins involved in haemin and haemoglobin-iron utilization. Mol Microbiol 53:1209-1220.

499. Wendland J, Philippsen P (2001) Cell polarity and hyphal morphogenesis are controlled by multiple rho-protein modules in the filamentous ascomycete Ashbya gossypii. Genetics 157:601-610.

500. Whiteway M, Bachewich C (2007) Morphogenesis in Candida albicans. Annu Rev Microbiol 61:529-553.

501. Whiteway M, Oberholzer U (2004) Candida morphogenesis and host-pathogen interactions. Curr Opin Microbiol 7:350-357.

502. Wickes B, Staudinger J, Magee BB, Kwon-Chung KJ, Magee PT, Scherer S (1991) Physical and genetic mapping of Candida albicans: several genes previously assigned to chromosome 1 map to chromosome $R$, the rDNA-containing linkage group. Infect Immun 59:2480-2484.

503. Widom J (1998) Structure, dynamics, and function of chromatin in vitro. Annu Rev Biophys Biomol Struct 27:285-327.

504. Wieland J, Nitsche AM, Strayle J, Steiner H, Rudolph HK (1995) The PMR2 gene cluster encodes functionally distinct isoforms of a putative $\mathrm{Na}^{+}$pump in the yeast plasma membrane. Embo J 14:3870-3882.

505. Wild GE, Searles LE, Koski KG, Drozdowski LA, Begum-Hasan J, Thomson AB (2007) Oral polyamine administration modifies the ontogeny of hexose transporter gene expression in the postnatal rat intestine. Am J Physiol Gastrointest Liver Physiol 293:G453-460.

506. Wilson LS, Reyes CM, Stolpman M, Speckman J, Allen K, Beney J (2002) The direct cost and incidence of systemic fungal infections. Value Health 5:26-34.

507. Willert EK, Phillips MA (2008) Regulated expression of an essential allosteric activator of polyamine biosynthesis in African trypanosomes. PLoS Pathog 4:e1000183.

508. Wimalasena TT, Enjalbert B, Guillemette T, Plumridge A, Budge S, Yin Z, Brown AJ, Archer DB (2008) Impact of the unfolded protein response upon genome-wide expression patterns, and the role of Hac1 in the polarized growth, of Candida albicans. Fungal Genet Biol 45:1235-1247.

509. Winstall E, Sadowski M, Kuhn U, Wahle E, Sachs AB (2000) The Saccharomyces cerevisiae RNA-binding protein Rbp29 functions in cytoplasmic mRNA metabolism. J Biol Chem 275:21817-21826.

510. Winter D, Podtelejnikov AV, Mann M, Li R (1997) The complex containing actinrelated proteins Arp2 and Arp3 is required for the motility and integrity of yeast actin patches. Curr Biol 7:519-529.

511. Wirth R, Bauer J, Sieber C (2008) Necrotizing Candida infection after percutaneous 
endoscopic gastrostomy: a fatal and rare complication. JPEN J Parenter Enteral Nutr 32:285-287.

512. Wittschieben BO, Fellows J, Du W, Stillman DJ, Svejstrup JQ (2000) Overlapping roles for the histone acetyltransferase activities of SAGA and elongator in vivo. Embo J 19:3060-3068.

513. Wolfger H, Mamnun YM, Kuchler K (2001) Fungal ABC proteins: pleiotropic drug resistance, stress response and cellular detoxification. Res Microbiol 152:375-389.

514. Workman JL, Kingston RE (1998) Alteration of nucleosome structure as a mechanism of transcriptional regulation. Annu Rev Biochem 67:545-579.

515. Wu J, Suka N, Carlson M, Grunstein M (2001) TUP1 utilizes histone H3/H2Bspecific $H D A 1$ deacetylase to repress gene activity in yeast. Mol Cell 7:117-126.

516. Wu J, Suka, N., Carlson, M. \& Grunstein, M. (2001) TUP1 utilizes histone H3/H2Bspecific HDA1 deacetylase to repress gene activity in yeast. Mol. Cell 7,: 117-126

517. Wu PY, Winston F (2002) Analysis of Spt7 function in the Saccharomyces cerevisiae SAGA coactivator complex. Mol Cell Biol 22:5367-5379.

518. Xu D, Jiang B, Ketela T, Lemieux S, Veillette K, Martel N, Davison J, Sillaots S, Trosok S, Bachewich C, Bussey H, Youngman P, Roemer T (2007) Genome-wide fitness test and mechanism-of-action studies of inhibitory compounds in Candida albicans. PLoS Pathog 3:e92.

519. Xu W, Edmondson DG, Evrard YA, Wakamiya M, Behringer RR, Roth SY (2000) Loss of Gcn5I2 leads to increased apoptosis and mesodermal defects during mouse development. Nat Genet 26:229-232.

520. Xu Z, Cao YB, Zhang JD, Cao YY, Gao PH, Wang DJ, Fu XP, Ying K, Chen WS, Jiang YY (2005) cDNA array analysis of the differential expression change in virulence-related genes during the development of resistance in Candida albicans. Acta Biochim Biophys Sin (Shanghai) 37:463-472.

521. Yamagoe S, Kanno T, Kanno Y, Sasaki S, Siegel RM, Lenardo MJ, Humphrey G, Wang Y, Nakatani Y, Howard BH, Ozato K (2003) Interaction of histone acetylases and deacetylases in vivo. Mol Cell Biol 23:1025-1033.

522. Yan L, Zhang J, Li M, Cao Y, Xu Z, Cao Y, Gao P, Wang Y, Jiang Y (2008) DNA microarray analysis of fluconazole resistance in a laboratory Candida albicans strain. Acta Biochim Biophys Sin (Shanghai) 40:1048-1060.

523. Yang XJ (2004) The diverse superfamily of lysine acetyltransferases and their roles in leukemia and other diseases. Nucleic Acids Res 32:959-976.

524. Yang XJ, Gregoire S (2005) Class II histone deacetylases: from sequence to function, regulation, and clinical implication. Mol Cell Biol 25:2873-2884.

525. Yang XJ, Seto E (2003) Collaborative spirit of histone deacetylases in regulating chromatin structure and gene expression. Curr Opin Genet Dev 13:143-153.

526. Yin Z, Wilson S, Hauser NC, Tournu H, Hoheisel JD, Brown AJ (2003) Glucose triggers different global responses in yeast, depending on the strength of the signal, and transiently stabilizes ribosomal protein mRNAs. Mol Microbiol 48:713-724.

527. Yordanov M, Dimitrova P, Patkar S, Saso L, Ivanovska N (2008) Inhibition of Candida albicans extracellular enzyme activity by selected natural substances and their application in Candida infection. Can J Microbiol 54:435-440.

528. Yoshida M, Kashiwagi K, Shigemasa A, Taniguchi S, Yamamoto K, Makinoshima $\mathrm{H}$, Ishihama A, Igarashi $\mathrm{K}$ (2004) A unifying model for the role of polyamines in bacterial cell growth, the polyamine modulon. J Biol Chem 279:46008-46013.

529. Zakikhany K, Naglik JR, Schmidt-Westhausen A, Holland G, Schaller M, Hube B (2007) In vivo transcript profiling of Candida albicans identifies a gene essential for interepithelial dissemination. Cell Microbiol 9:2938-2954.

530. Zanelli CF, Valentini SR (2005) Pkc1 acts through Zds1 and Gic1 to suppress growth and cell polarity defects of a yeast elF5A mutant. Genetics 171:1571-1581.

531. Zhang L, Eugeni EE, Parthun MR, Freitas MA (2003) Identification of novel histone post-translational modifications by peptide mass fingerprinting. Chromosoma 112:77-86.

532. Zhang M, MacDonald Al, Hoyt MA, Coffino P (2004) Proteasomes begin ornithine 
decarboxylase digestion at the $C$ terminus. J Biol Chem 279:20959-20965.

533. Zhang M, Pickart CM, Coffino P (2003) Determinants of proteasome recognition of ornithine decarboxylase, a ubiquitin-independent substrate. Embo J 22:1488-1496.

534. Zhang T, Li W, Li D, Wang Y, Sang J (2008) Role of CaECM25 in cell morphogenesis, cell growth and virulence in Candida albicans. Sci China C Life Sci 51:362-372.

535. Zhang W, Bone JR, Edmondson DG, Turner BM, Roth SY (1998) Essential and redundant functions of histone acetylation revealed by mutation of target lysines and loss of the Gcn5p acetyltransferase. Embo J 17:3155-3167.

536. Zhang X, De Micheli M, Coleman ST, Sanglard D, Moye-Rowley WS (2000) Analysis of the oxidative stress regulation of the Candida albicans transcription factor, Cap1p. Mol Microbiol 36:618-629.

537. Zhang $\mathrm{Y}$, Sun ZW, Iratni R, Erdjument-Bromage $\mathrm{H}$, Tempst $\mathrm{P}$, Hampsey $\mathrm{M}$, Reinberg D (1998) SAP30, a novel protein conserved between human and yeast, is a component of a histone deacetylase complex. Mol Cell 1:1021-1031.

538. Zhang Z, Reese JC (2004) Redundant mechanisms are used by Ssn6-Tup1 in repressing chromosomal gene transcription in Saccharomyces cerevisiae. J Biol Chem 279:39240-39250.

539. Zhao R, Daniels KJ, Lockhart SR, Yeater KM, Hoyer LL, Soll DR (2005) Unique aspects of gene expression during Candida albicans mating and possible $\mathrm{G}(1)$ dependency. Eukaryot Cell 4:1175-1190.

540. Zhao X, Oh SH, Cheng G, Green CB, Nuessen JA, Yeater K, Leng RP, Brown AJ, Hoyer LL (2004) ALS3 and ALS8 represent a single locus that encodes a Candida albicans adhesin; functional comparisons between Als $3 p$ and Als $1 \mathrm{p}$. Microbiology 150:2415-2428.

541. Zhao X, Oh SH, Yeater KM, Hoyer LL (2005) Analysis of the Candida albicans Als $2 p$ and Als $4 p$ adhesins suggests the potential for compensatory function within the Als family. Microbiology 151:1619-1630.

542. Zhao X, Pujol C, Soll DR, Hoyer LL (2003) Allelic variation in the contiguous loci encoding Candida albicans ALS5, ALS1 and ALS9. Microbiology 149:2947-2960.

543. Zheng X, Wang Y, Wang Y (2004) Hgc1, a novel hypha-specific G1 cyclin-related protein regulates Candida albicans hyphal morphogenesis. Embo J 23:1845-1856.

544. Znaidi S, De Deken X, Weber S, Rigby T, Nantel A, Raymond M (2007) The zinc cluster transcription factor Tac1p regulates PDR16 expression in Candida albicans. Mol Microbiol 66:440-452.

545. Zordan RE, Miller MG, Galgoczy DJ, Tuch BB, Johnson AD (2007) Interlocking transcriptional feedback loops control white-opaque switching in Candida albicans. PLoS Biol 5:e256.

546. Zurita-Martinez SA, Cardenas ME (2005) Tor and cyclic AMP-protein kinase A: two parallel pathways regulating expression of genes required for cell growth. Eukaryot Cell 4:63-71. 